\title{
A Precision Test of Charge Independence of Hadronic Interactions
}

\author{
Marina Artuso*
}

\section{DISCLAIMER}

This report was prepared as an account of work sponsored by an agency of the I Inited States Government. Neither the United States Government nor any agency thereof, nur any of their employees, makes any warranty, express or implied, or assumes any icgal liability or responsibility for the accuracy, completeness, or usefulness of uny informatior. apparatus, product, or process disclosed, or represents that its use wuld no: infringe privately buned rights. Reference herein to any specific commercial product. prekess or service by trade name, tradeinark, manufacturer, or otberwise does not necessarily constitute or imply its endorscment, recommendation, or favoring by the United States Government or any agency thereof. The views and opinions of authors expressed herein do not recessarily state or reflect those of the United Sta'es Government or any agency thereof.

-Guest Scientist at Los Alamos. Department of Physics and Astronomy, Northwestern University, Evanston, IL 60201.
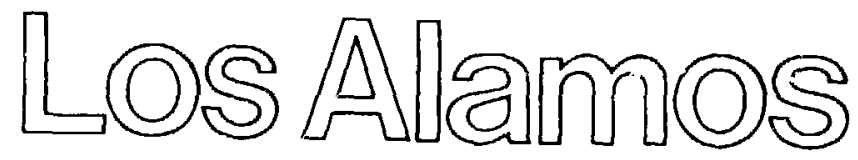


\section{Preface}

I would like to thank my thesis advisor Prof. Kamal K. Seth for his support and advice througout this project.

Special thanks are due to my collaborators Dr. R. Soundranagayam and Dr. Brett Parker, who provided many helpful discussions and a friendly work environment.

I would like to thank J. H. Lin for the great help that he provided in processing the manuscripi.

I would also like to thank Dr. L. Rosen, Director of LAMPF, for his help in an carly scheduling of this experiment, and the MP10 staff, in particular J. McClelland, K. Jones and J. Amann for their help during the experiment and for interesting discussions.

Finally special thanks are due to Maggie Eustler, Maxine Joppa and Roberta Marinuzzi, who made my st at Los Alamos a very pleasant one. 


\section{Contents}

Preface $\quad$ v

Contents vi vi

List of Figures $\quad$ ix

List of Tables $\quad$ xiv

Abstract $\quad$ xvi

1 Introduction $\quad 1$

1.1 Symmetries in physics .................... 1

1.2 Isospin Invariance . . . . . . . . . . . . . . . . 3

1.3 Isospin in the context of meson theories. . . . . . . . . . . . 8

1.4 Isospin in the context of QCD . . . . . . . . . . . . 11

1.5 A scale for charge symmetry and charge independence violation. . 15

1.5.1 Masses of Isospin Multiplets. . . . . . . . . . . 15

1.5.2 Isospin Mixing ................. 18

1.6 Experimental evidence for charge symmetry. . . . . . . . . . . . 20

1.6.1 Nucleon - Nucleon scattering. . . . . . . . . . . . 20

1.6.2 Pion - Deuteron Total Cross Sections. . . . . . . . . . . . . 22

1.6.3 Yion - Deuteron Elastic Scallering. . . . . . . . . 23

1.6.4 $\pi-{ }^{3} \mathrm{H}$ and $\pi-{ }^{3} \mathrm{He}$ Scaitering. . . . . . . . . . . . 24

1.6.5 $\pi-{ }^{4} \mathrm{He}$ Scattering. ................. 26 
1.6.6 The Reaction $\mathrm{d}+\mathrm{d} \rightarrow{ }^{4} \mathrm{He}+\pi^{0} \ldots \ldots \ldots \ldots$

1.6.7 The Rewction $n p \rightarrow \mathrm{d} \pi^{\circ} \ldots \ldots \ldots \ldots \ldots$

1.6.8 Neutron-Proton Elastic Scattering. . . . . . . . . . 28

1.7 Charge Indeperdence . . . . . . . . . . . . . . . . 30

1.7.1 Low Energy np Scattering . . . . . . . . . . . . . . 30

1.7.2 Pion-nuclcon total cross section . . . . . . . . . . 31

1.7.3 The Reactions $\mathrm{p}+\mathrm{d} \rightarrow \pi^{+}+{ }^{3} \mathrm{H}$ and $\mathrm{p}+\mathrm{d} \rightarrow \pi^{\circ}+{ }^{3} \mathrm{He} \ldots 33$

1.8 Criteria for choice of an experiment. . . . . . . . . . 35

1.8.1 Choice of the incident energy. . . . . . . . . 37

1.8.2 Choice of the Kinematical point. . . . . . . . 38

1.8.3 Choice of the target. . . . . . . . . . . . 41

1.9 Survey of previous measurements of the ratio $R \ldots \ldots \ldots$

1.10 The present experiment. . . . . . . . . . . . 50

1.10.1 Difficulties in interpretation. . . . . . . . . 51

2 The experimental method. $\quad 52$

2.1 The beam. . . . . . . . . . . . . . . . 52

2.2 The Target. . . . . . . . . . . . . . . 56

2.3 The HRs spectrometer. . . . . . . . . . . . . . . 59

2.4 Signal processor for the HRS detector system. . . . . . . . 61

2.5 The particle identification system. . . . . . . . . . 66

2.6 Monte Carlo simulation of detected particle propagation through the spectrometer ................... 69

3 Data analysis and results. $\quad 83$

3.1 The analysis and calibration software. . . . . . . . . 83

3.2 The HRS event reconstruction. . . . . . . . . . . . . 84

3.3 Calibrations. . . . . . . . . . . . . . . . 90

3.4 The good event test. . . . . . . . . . . . . . . 98

3.5 The method of analysis. . . . . . . . . . . . 108 
3.6 Calculation of $R(0)$ and $A_{\mathrm{y} 0}(0) \ldots \ldots \ldots \ldots \ldots$

3.7 Error analysis. . . . . . . . . . . . . . . . . . . 119

3.8 Discussion of Experimental Results. . . . . . . . . . . . 128

4 Theoretical analysis and conclusions. $\quad 130$

4.1 The calculation of Köhler. . . . . . . . . . . . . . . 130

4.2 Theories of pion production. . . . . . . . . 135

4.3 Conclusions. . . . . . . . . . . . . . . . . . . 145

Appendix A. $\quad 152$

$\begin{array}{lr}\text { Apperdix B. } & 154\end{array}$

Appendix C. $\quad 157$

$\begin{array}{ll}\text { Appendix D. } & 160\end{array}$ 


\section{List of Figures}

1.1 Feynman diagrams for one pion exchange nucleon-nucleon potential. .................... 8

1.2 Examples of ' $\pi-\eta$ ' and the ' $\rho-\omega^{\prime}$ mixing diagrams. . . . . . . . 10

1.3 Isospin mixing matrix elements inferred from isospin forbidden Fermi $\beta^{\mathrm{t}}$ decays.(from Ref. 34 ) . . . . . . . . . . . . . . 18

1.4 Difference in $\sigma_{t}\left(\pi^{-} d\right)$ and $\sigma_{t}\left(\pi^{+} d\right)$. (from Ref. 45) . . . . . . . 22

1.5 Asymmetry $\mathrm{A}(\theta)$ in $\%$ for $\pi^{+}, \pi^{-}$elastic scattering cross sections (from Ref. 49). . . . . . . . . . . . . 23

1.6 Ratios for $\pi^{ \pm}-{ }^{3} \mathrm{H}\left({ }^{3} \mathrm{He}\right)$ elastic scattering. . . . . . . . . . . 25

1.7 Most recent results for the super-ratio $\mathrm{R}$ defined in Fig. 1.6 (from Ref. 53) ................... . . 25

1.8 Asymmetry $\mathrm{A}(\theta)$ for $\pi^{ \pm}$elastic scattering from ${ }^{4} \mathrm{He}$ (from Ref. 54), 26

1.9 Percent deviation $\left[3 \sigma\left(\pi^{-} p\right)-\sigma\left(\pi^{+} p\right) / \sigma\left(\pi^{+} p\right)\right] \cdot 100 \ldots \ldots 32$

1.10 Differential cross section for the reaction $p+d \rightarrow{ }^{3} \mathrm{H}+\pi^{+}$. The data are from Refs. 68-72 and 90. ......... 40

1.11 Analyzing power for the reaction $p+d \rightarrow{ }^{3} \mathrm{H}+\pi^{+}$at $\mathrm{T}_{p}=700$ MeV. (from Ref. 90) .................. . 40

1.12 Kinematics for the reaction $\mathrm{p} \mathrm{d} \rightarrow{ }^{3} H\left({ }^{3} \mathrm{He}\right) \pi^{+}\left(\pi^{0}\right)$ as measured by Crewe et al.(Ref. 75, 76).

1.13 Kinematics for the reaction $\mathrm{p} \mathrm{d} \rightarrow{ }^{3} \mathrm{H}\left({ }^{3} \mathrm{He}\right) \pi^{+}\left(\pi^{0}\right)$ as measured by llarting et al.(Ref. 77,78$)$.

1.14 Kinematics for the reaction $\mathrm{p} \mathrm{d} \rightarrow{ }^{3} H\left({ }^{3} \mathrm{He}\right) \pi^{+}\left(\pi^{0}\right)$ as measured by Booth et al.(Ref. 79) . . . . . . . . . . . . . 
1.15 Kinematics for the reaction $\mathrm{p} d-{ }^{3} H\left({ }^{3} H e\right) \pi^{+}\left(\pi^{0}\right)$ as measured by Silverman el al.(Rer. 80)

1.16 Kincmatics for the reaction p d $-{ }^{3} I\left({ }^{3} I e\right) \pi^{+}\left(\pi^{0}\right)$ for the present experiment.

2.1 Schematic view of the LAMPL accelerator. . . . . . . 53

2.2 Schematic yew of line C. . . . . . . . . . . . . 54

2.3 Seliematic of $L D_{2}$ target under 15 psi pressure. . . . . . . . 58

2.4 Schematic representation of the IIRS detector system. Scintillators $S_{1}$ and $S_{3}$ were not used in the present experiment and were moved out of position. . . . . . . . . . . . . . . . 60

2.5 Block diagram of the HRS signal processor. . . . . . . . . . 63

2.6 Block diagram of the IIRS slow gate logic. . . . . . . . . 65

2.7 Experimental angular spectra for the reactions $\mathrm{D}\left(\mathrm{p},{ }^{3} \mathrm{H}\right) \pi^{+}$and $\mathrm{D}\left(\mathrm{p},{ }^{3} \mathrm{He}\right) \pi^{0}$ as compared with the angular acceptance of the HRS spectrometer for an inclastic reaction. ................ 70

2.8 Coordinate system for the two-body reaction. . . . . . . . 71

2.9 Coordinates $\left(\mathrm{x}_{f}, \theta_{f}, \mathrm{y}_{f}, \phi_{f}\right)$ at the front plane for the reaction $\mathrm{p}+\mathrm{d}$ $\rightarrow^{3} \mathrm{H}+\pi^{+} \ldots \ldots \ldots \ldots \ldots$

2.10 Coordinates $\left(\mathrm{x}_{f}, 0_{f}, \mathrm{y}_{f}, \phi_{f}\right)$ at the front plane for the reaction $\mathrm{p}+\mathrm{d}$ $\rightarrow^{3} \mathrm{He}+\pi^{0} \ldots \ldots \ldots \ldots \ldots \ldots$

2.11 Coordinate $x_{f}$ at the front plane for the reaction $p+d \rightarrow p^{\prime}+X . \quad 77$

2.12 Normalized missing mass spectra from Monte Carlo simulation (continuous line) and from experimental data for the three reactions $d\left(p,{ }^{3} l l\right), d\left(p,{ }^{3} l\right.$ le $)$ and $d\left(p, p^{\prime}\right)$.

2.13 Normalized missing mass spectira from Monte Carlo simulation (continuous line) and from experimental data for elastic scattering from

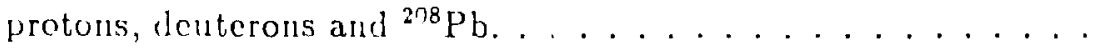


2.14 Normalized scaltering angle spectra from the Monte Carlo simulation and experimental data. . . . . . . . . . . . . . .

3.1 Flow-diagram of the event reconstruction performed by the HRS event processor

3.2 Geometry for the determination of the corrected extrapolated position of a particle track through the $\mathrm{x}$ drift chambers. . . . . 87

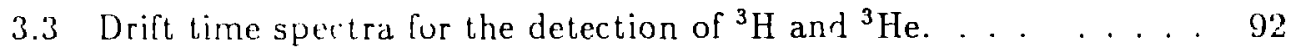

3.4 Drift distance spectra for ${ }^{3} \mathrm{Il}$ (X chambers). . . . . . . . . 93

3.5 Drift distance spertra for ${ }^{3}$ Il (Y chambers). . . . . . . . . . 94

3.6 Drift distance spectra for ${ }^{3} \mathrm{He}$ (X chambers). . . . . . . . . 95

3.7 Drift distance spectra for ${ }^{3} \mathrm{He}(\mathrm{Y}$ chambers $) \ldots \ldots \ldots \ldots 6$

3.8 A typical missing mass spectrum for the reaction ${ }^{208} \mathrm{~Pb}(\mathrm{p}, \mathrm{p})^{208} \mathrm{~Pb} . \quad 97$

3.9 A typical proton $\delta$ (momentum deviation) spectrum for the reaction ${ }^{208} \mathrm{~Pb}(\mathrm{p}, \mathrm{p})^{208} \mathrm{~Pb} \ldots \ldots \ldots \ldots \ldots \ldots$

3.10 Spectra for the wire identification variables in the dispersion direction.

$3.11 X_{f}$ distribution of the events corresponding to 0 (top), 1 (middle), and 2 (bottom) wire difference in extrapolated position for the front chambers $\mathrm{C}_{1}, \mathrm{C}_{2} \ldots \ldots \ldots \ldots \ldots \ldots \ldots \ldots$

$3.12 X$, distribution of the events corresponding to 0 (top), 1 (middle), 2 (bottom) wire difference in extrapolated position for the rear charnbers $\mathrm{C}_{3}, \mathrm{C}_{4}$.

3.13 Scattering angle spectra gated by the conventi $\sim$ nal good event test. 103

3.14 Nornalized $x$, distributions for the reactions $\mathrm{D}\left(\mathrm{p},{ }^{3} \mathrm{H}\right) \pi^{+}$and $\mathrm{D}\left(\mathrm{p},{ }^{3} \mathrm{He}\right) \pi^{0}$ gated by the 'extended good event' test. . . . . . . . . . . . . . 104

3.15 Scattering angle spectra gated by the 'extended good event' test. Compare with Fig. 3.13 . . . . . . . . . . . 105 
3.16 Dot phots for ${ }^{3} 11$ and ${ }^{3} 11$ e identification, abscissa are pulse heights and ordinates are time of flight. (box 1 contains the particles of interest in both cases.) . . . . . . . . . . . . . 106

3.17 Dot plot for XANG cut. The abscissa is $x_{f}$ and the ordinate is related to the angle $0_{1}$. Box 4 defines the fiducial space. . . . . . 107

3.18 Comparison of the yields in the tail region the missing mass spectra for normal and reverse polarization

3.19 $A_{y 0}$ as a function of missing mass for the two reactions (no background subtraction has been done). . . . . . . . . . . 110

320 Comparison between the tails of the normalized missing mass spectra for ${ }^{3}$ II and ${ }^{3}$ lle detection. . . . . . . . . . . . . . 112

3.21 Missinf mass spectra for the two reactions. The dotted curves indicate the background level.

$3.22 A_{y 0}$ as a function of missing mass for the reactions $\vec{p}+d \rightarrow^{3} H+\pi^{+}$ and $\vec{p}+d \rightarrow{ }^{3} M e+\pi^{0}$ (after background subtraction. . . . . 118

3.23 Measured geometrical factors $G_{\text {hor }}$ for Line-C polarimeter. The open circles correspond to "He detection and the filled circles correspond to ${ }^{3} \mathrm{II}$ detection. . . . . . . . . . . . . .

$3.24 x_{f}$ distribution of events for ${ }^{3} \mathrm{H}$ and ${ }^{3} \mathrm{He}$ within the gates $\Delta \theta_{1}$ and $\Delta 0_{2} \ldots \ldots \ldots \ldots \ldots \ldots$

4.1 The Impulse approximation for the $p d \rightarrow t \pi^{+}$reaction (from Rer. 94).

4.2 Differential cross sections for the $p d \rightarrow t \pi^{+}$reaction at $800 \mathrm{McV}$. l'he experimental data are from Ref. 90. The theoretical curves are from Refs. 94 and 98 .

1.3 The basic pion production mechanism considered by Green and collaborators. "The bubble in the diagram on the lect is "expanded" on the right to show all the contributions. 
$4.4 p d \rightarrow t \pi^{+}$differential cross section predictions from the caiculations of Green and collaborators (from Ref. 97). . . . . . . . . 139

4.5 Diagrams for the amplitudes associated with the reaction $p+d \rightarrow$ ${ }^{3} \mathrm{H}+\pi^{+}$according to Laget and LeColley (Ref. 94). . . . . . . . 141

4.6 Analyzing power for the $p d^{\prime} \rightarrow \mathrm{t} \pi^{+}$reaction at $800 \mathrm{MeV}$. The experimental data are from Ref. 90. The theoretical curve is from Ref. 98. . . . . . . . . . . . . . . . . . 143

4.7 Laget and LeColley's prediction for the ratio $\sigma\left({ }^{3} \mathrm{HI}+\pi^{+}\right) / \sigma\left({ }^{3} \mathrm{He}+\pi^{0}\right)$ at $\mathrm{T}_{\mathrm{p}}=730 \mathrm{MeV} \ldots \ldots \ldots \ldots \ldots \ldots \ldots \ldots \ldots$

4.8 Laget and LeColley's prediction for the difference in analyzing power between $\vec{p}+d \rightarrow{ }^{3} \mathrm{H}+\pi^{+}$and $\vec{p}+d \rightarrow{ }^{9} \mathrm{He}+\pi^{0} \ldots \ldots 144$

B.1 Energy level diagram of the hydrogen atom for principal quantum number $n=2 \ldots \ldots \ldots \ldots \ldots \ldots \ldots \ldots$ 


\section{List of Tables}

1.1 Evaluations of ratios between merent alnetsa mowes of the lin.t. quarks.

1.2 Masses of dilferent charge members of baryon and meson multiplets 16

1.3 Coulomb energy shift and correction terms (in MeV) (from Ref. $33)$

1.4 Isospin mixed doublets (from Ref. 34) . . . . . . . . . .

1.5 Low energy nucleon-nucleon scattering parameters: effective lengths and effective ranges .....................

1.6 Theoretical resolution of the contributions to $\Delta a=a_{n p}-\left\langle a_{n n}, a_{p p}\right\rangle$

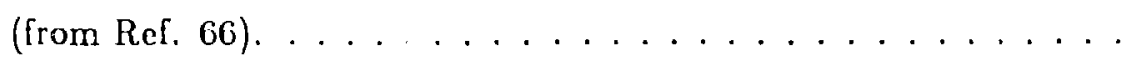

2.1 Thickness distribution as a function of the coordinate $\rho \ldots \ldots 8$

2.2 Geometrical characteristics of the detectors associated with the HRS magnetic spectrometer. . . . . . . . . ...... 59

2.3 Kinetic energy of background particles corresponding to rigidity $\left(\mathrm{p}_{0} / \mathrm{z}\right)$ range $=1486.3-1523.9 \mathrm{MeV} / \mathrm{c} / \mathrm{z}$ for ${ }^{3} \mathrm{H}$ and $739.7-758.4 \mathrm{MeV} / \mathrm{c} / \mathrm{z}$ for ${ }^{3}$ He. . . . . . . . . . . . . . . . . . . . .

2.4 Time of flight and energy loss ranges for signal and background particles which enter the rigidity acceptance of the spectrometer.

2.5 Input parameters for the Monte Carlo simulation of the reactions $\mathrm{d}\left(\mathrm{p},{ }^{3} \mathrm{H}\right) \pi^{+}$and $\mathrm{d}\left(\mathrm{p},{ }^{3} \mathrm{He}\right) \pi^{0} \ldots \ldots \ldots \ldots$

2.6 Monte Carlo results for detector acceptance in $\%$ of the particles which arrive at a detector. . . . . . . . . . . . . . 
2.7 Integral efficiency for the two angular gates employed in the analysis from the Monte Carlo simulation. (Angles are referred to the

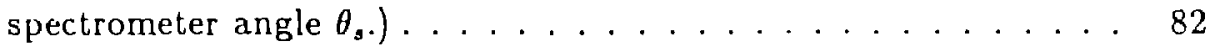

3.1 Fraction of events passing the wire identification test for the drift chambers used in our experiment. . . . . . . . . . . . 100

3.2 Average angles for the observables measured. . . . . . . . . 11 t

3.3 Parameters employed to normalize experimental yields. . . . . 117

3.4 Summary of error analysis. . . . . . . . . . . . . . . 125

3.5 Sumimary of Experimental results(I). 'The errors are statistical only. 129

3.6 Summary of experimental results (.j.). On each quantity, the first errors are statistical, and the secord errors (in parentheses) are systematic. . . . . . . . . . . . . . . . . . . 129 


\section{Abstract}

A precision test of clarge independence of hadronic interactions

Broken symmetries are among the richest sources of information about the fundamental interactions: the renewed interest in the study of isospin non-conservation by strong forces is closely related to the effort of understanding some properties of nuclear systems in terms of their basic degrees of freedom.

The nope is to be able to relate the pattern of the dynamical breaking of this sysmmetry to the mass spectrum of light quarks: to this purpose a more detailed phenomenological knowledge must be provided by a new generation of experiments.

These considerations motivated us to perform a precision test of charge independence of strong nuclear interactions through a measurement of the parameters $\triangle A_{y^{0}}(\theta)=A_{y^{0}}\left(\theta,{ }^{3} H\right)-A_{y^{0}}\left(\theta,{ }^{3} H e\right)$, (the difference in analyzing power), and $R \equiv d \sigma\left(\theta,{ }^{3} H\right) / d \sigma\left(\theta,{ }^{3} H e\right)$ for the two reactions:

$$
\begin{aligned}
& \vec{p}+d \longrightarrow^{3} H+\pi^{+} \\
& \vec{p}+d \longrightarrow^{3} H e+\pi^{0}
\end{aligned}
$$

The observable $\Delta A_{y 0}$ is particularly relevant as it probes the spin dependent term of the symmetry breaking interaction, on which so far almost no empirical evidence is available.

The experiment has been performed at the Los Alamos Meson Physics Facility, using the N-type poiarized proton beam $\left(\mathrm{T}_{\vec{p}}=733 \mathrm{MeV}\right)$, and detecting the charged heavy particle in the HRS magnetic spectrometer.

The final results are:

$$
\Delta A_{y 0}=A_{y 0}\left({ }^{3} \mathrm{H}\right)-A_{y 0}\left({ }^{3} \mathrm{He}\right)=0.3930-0.3996=-0.0066 \pm 0.0040 \pm(0.0018)
$$

and

$$
R=2.193 \pm 0.007 \pm(0.027)
$$

where the first errors are statistical and the second systematic. 


\section{Chapter 1}

\section{Introduction}

\subsection{Symmetries in physics}

The concept of symmetry can be considered as one of the 'backbones' of human culture, as has been pointed out by Weyl in his classic book on the topic ${ }^{1}$. Symmetries have always played a fundamental role in our effort to understand the physical world around us. There is a definite trend in the evolution of physics to look for deeper and deeper connections between the dynamical content of theories and the underlying symmetries.

Before the advent of quantum mechanics and quantum field theories of the elementary particles and their interactions, symmetries had a miie geomet,rical nature: the classical model for them ${ }^{1}$ was the left-right specularity exhibited by many structures which are part of our daily life. Geometrical symmetries can be classified into two main groups: discrete symmetries, like parity or time-reversal, and continuous ones, like trans'ations or rotations.

The attempt to put these concepts in more quantitative terms lead to the development of group theory. Some fields of physics which study structures exhibiting a high degree oi regularity, like crystallography, are based on these mathematical methods.

A deeper dynamical role of symmetry principles was actually built in the Lagrangian and Hamiltonian formulation of classical mechanics and the associated field theory: the connection between invariance of the Lagrangian under 
some transformations and the concept of constants of motion. This connection became a fundamental concept in quantum mechanics, allowing the identification of the 'good quantum numbers' associated with a physical system. Besides the space-time variables, which are suitable for the description of the macroscopic world, quantum systems have new degrees of freedom. Transformations in some of these 'quantum' subspaces which leave a given system invariant are classified as 'internal' symmetries.

From the classical theory of fields comes a theorem which has become the starting point for the devclupment of the 'gauge theories' of the elementary interactions. ${ }^{2}$ It states that to any continuous one-parameter symmetry of the Lagrangian is associated a conserved current. The 3 -space integral of this current can be identified with a conserved charge. The study of the elementary particles and their interactions has led to a further distinction between 'global' and 'local' symmetries, that is between transformations independent of the space-time point at which they are performed and transformations which are functions of spacetine. The latter group represents the actual core of the 'gauge' theorics of the elementary interactions. Here the symmetry assumed dictates the structure of the coupling between the various fieldis.

The 'standard-model' of the strong and electro-weak interactions marks the great success of this theoretical approach, which was originally proposed by Yang and Mills $^{3}$ in an attempt to describe the interaction between a proton and a neutron with a Lagrangian invariant under SU(2) 'gauge' transformation.

The discovery of these powerful tools awakened the everlasting dream of physicists: a unified description of all the fields and interactions which are known to exist. Theories, so far based more on aesthetic appeal than on empirical evidence, generally known as Grand Unified Theories, have been developed.

This short journey among the more ambitious flights of the modern theoretical physics is bound to end with a reference to supersymmetry, which 'is the supreme symmetry: it unifies space-time symmetries with internal symmetries, 
fermions with bosons and gravity with matter'.

As far as global symmetries are concerned, they are certainly less fundamental aspects of our present understanding of the microworld. They are not required by any theoretical principle: no revolution would occur in our understanding of the basic interactions if some experimental data would prove that they are not exact. However, in the development of phenomenological models which try to describe complex systems, as it is the case, for instance, in nuclear phyisics, global symmetries act as fundamental constraints. It is therefore of extreme interest to investigate whether they are exact or approximate symmetries, and, if they are approximate, to reach a deeper understanding of the extent and the pattern of the symmetry breaking component of the interaction.

As it will be pointed out in a following section, a great theoretical effort is presently involved in a reformulation of the nuclear theory by including the most relevant features of $\mathrm{QCD}$, which has a good chance of being the correct theory of the strong interartion. The argument developed above therefore suggests that a wider phenomenological knowledge of the internal symmetries of hadronic systems has to be achieved.

\subsection{Isuspin Invariance}

Isospin was the first internal symmetry postulated in nuclear physics: soon after the discovery of the neutron, Heisenberg ${ }^{5}$ proposed the 'sucleon' as the elementary particle sensitive to the strong force. In other words, Heisenberg conjectuied that in a hypothetical world in which only the forces respunsible for binding nucleons in nuclei are present, there are no dynamical effects which are able to distinguish protons from neutrons. In the isospin formalism they correspond to the fundamental representation of an internal SU(2) group. A rotation in this space is generated by the operator $R_{\tau}=\exp (i \hat{0} \hat{n} \cdot \vec{\tau} / 2)$, where $\hat{n}$ is the unit vector which gives the direction of rotation and $\vec{\tau}=\left(\tau_{1}, \tau_{2}, \tau_{3}\right)$ is the set of Pauli matrices, i.e. the generators of the $\mathrm{SU}(2)$ group in the fundamental spinor representation. 
The two basis states, corresponding respectively to the proton and the neutron, are the eigenstates of the $\tau_{3}$ operator with eigenvalues +1 and -1 respectively. Soon after Heisen'erg's conjecture, it was recognized that a nuclear Hamiltonian dependent upon space and spin coordinates of the interacting particles but not upon their projection along the 3-axis in isospin space was consistent with all the known systematics. ${ }^{\sigma}$

After the discovery of the pion ${ }^{7}$, which took place soon after Yukawa ${ }^{8}$ proposed that some 'exchanged particle' was the mediator of nuclear interaction, the isospin formalism was generalized to encompass also the newly discovered mesons. The pions were required to carry an isospin quantum number to generate a nucleon-nucleon interaction consistent with the invariance property discussed above. It is interesting to note that it was exactly the equality in range of the proton-neutron and proton-proton or neutron-neutron interaction which suggested the existence of a neutral pion or, in other words, that the pion is a triplet in isospin space. $^{9}$

There are two fundamental symmetries which can be recognized in isospin space. The first one, known as 'charge independence', corresponds to a rotational inrariance in this internal space, in other words, the interaction must be a scaiar as far as isospin is concerned. A less restrictive invariance is associated with the 'charge symmetry operator' $\mathrm{P}_{c t}=\mathrm{e}^{i \pi \tau_{2}}$, which corresponds to a rotation of 180 degrees around the $\hat{2}$ axis. Its effect is the transformation of a proton into a neutron and vice versa: in a nucleus $\tau_{2}$ is substiluted by $T_{2}=\sum_{i=1}^{N} \tau_{2}(i)$ : it has the effect of reversing the $\hat{z}$ isospin projections of all the nucleons. Charge independence guarantees charge symmetry but charge independence breaking can take place even if charge symmetry is exact. The charge operator, associated with the Coulomb interaction, has an isovector component, as it is defined as $Q=\mathrm{Y} / 2+T_{3}$, where $\mathrm{Y}$ stands for the hypercharge and $T_{3}$ stands for the total isospin projection of the hadronic system. It breaks charge independence and 
charge symmetry as the commutator

$$
\left[e^{i \pi T_{2}}, T_{3}\right]=2 e^{i \pi T_{2}} \cdot T_{3}
$$

which in the general case is different from 0 . However, $T_{3}=0$ for 'self-conjugate' systems, with equal number of protons and neutrons, and therefore the charge and isospin parity eigenvalues can be specified simultaneously.

A general pattern which can be identified in the evolution of physics is the fact that the identification of some new symmetry underlying a given interaction is always followed by an experimental effort with the purpose of establishing whether this symmetry is exact or not on the basis of a stronger empirical evidence. If the symmetry turns out to be an approximate one, irterest focuses on determining the extent and the patterns of its violation. The first set of 'dedicated' experiments performed to test isospin symmetry were measurements of the parameters characterizing the low energy nucleon-nucleon scattering and gave some evidence that this symmetry is not exact: these experiment will be discussed later in this chapter.

Henley ${ }^{10-12}$ performed a formal analysis of the possible patterns of isospin violation, identifying the structure of the pctertial operators associated with them. He recognized four categories of potential operators describing the nucleon-nucleon interaction in isospin space, corresponding to different transformation proporties with respect to isospin rotations:

$$
\left.V_{1}(i j)=A \mid 1+\vec{\tau}(i) \cdot \vec{\tau}(j)\right\}
$$

This is an isoscalar potential which commutes with all the generators associated with this global symmetry: it therefore represents the bulk of the nuclear interaction, conserving isospin quantum numbers. 


$$
V_{2}(i j)=B\left[\tau_{3}(i) \tau_{3}(j)-\frac{1}{3} \vec{\tau}(i) \cdot \vec{\tau}(j)\right]
$$

This is an isotensor operator which commutes with the charge symmetry operator $\mathrm{P}_{\text {cos }}$.

$$
V_{3}(i j)=C\left\{\tau_{3}(i)+\tau_{3}(j)\right\}
$$

This is an isovector term with the same structure as the isospin violating term of the Coulomb interaction, as the charge operator is given by $Q=\frac{Y}{2}+T_{3}$. It breaks isospin independence and isospin symmetry, but is unaffected by the interchange of any two nuclcons.

$$
V_{4}=D\left[\tau_{3}(i)-\tau_{3}(j)\right\}\{\vec{\sigma}(i)-\vec{\sigma}(j)] \cdot \vec{L}_{i j}+E[\vec{\tau}(i) \times \vec{\tau}(j)\}_{3}[\vec{\sigma}(i) \times \vec{\sigma}(j)] \cdot \vec{L}_{i j}
$$

This potential breaks all the possible symmetries in isospin space. It affects the np system but not the nn or pp systems. It should produce noticeable effects on the polazization observables associated with np pairs in free space or in nuclear environment.

For the sake of completeness we also mention the Coulomb interaction which is the most common agent of isospin breaking. It can be writter as.

$$
C_{i j}=\frac{e^{2}}{4 r_{i j}} \cdot\left\{1-\tau_{3}(i) \mid\left[1-\tau_{3}(j)\right]\right.
$$

It contains two terms which do not conserve isospin: the $\tau_{3}(i) \tau_{3}(j)$ term breaks charge independence but not charge symmetry, and the $\left[\tau_{3}(i)+\tau_{3}(j)\right\rfloor$ term which breaks both.

Unfortunately, it is difficult to draw definitive conclusions about the extent and the patterns of isospin breaking. Because of our lack of knowledge of the details of the hadronic interaction, it is generally not possible to extract the effects of the interference between the strong and electromagnetic terms in the scattering 
matrix in a model independent way. Moreover the knowledge of the existence of the isospin non-conserving electromagnetic interaction and the aesthetic appeal of isospin symmetry in the treatment of nuclear phenomenolory has somewhat biased the theoretical approach to the investigation of its validity. A clear example is the attitude toward the parameter $\Delta=m_{p}-m_{n}=-1.2395 \mathrm{MeV}$. In a sense this is ihe first indication that the symmetry under consideration is an approximate one; no theoretical calculation based on the assumption that $\Delta$ is purely of eleciromagnetic origin is able to reproduce even its sign. However, until the advent of QCD the value of $\Delta$ was considered an electromagnetic effect $t^{10}$, even if not understood. 


\subsection{Isospin in the context of meson theories.}

It is interesting to analyze the evolution of the concept of isospin conservation when the meson theory of nuclear force established itself as a successful model of hadronic interactions. In a sense isospin was still considered an intrinsically exact symmetry; all the manifestations of its breaking in nuclear phenomenology were still ascribed to the electromagnetic force.

In this theoretical framework, the nucleon-nucleon potential arises by the exchange of virtual mesons. The example of the pion exchange contribution to the nucleon-nucleon potential is illustrated in Fig. 1.1, which shows the relevant diagrams for OPEP (one pion exchange potential). As the mass of the exchanged particle determincs the range of the interaction mediated by it, this term describes the long range part of the interaction.

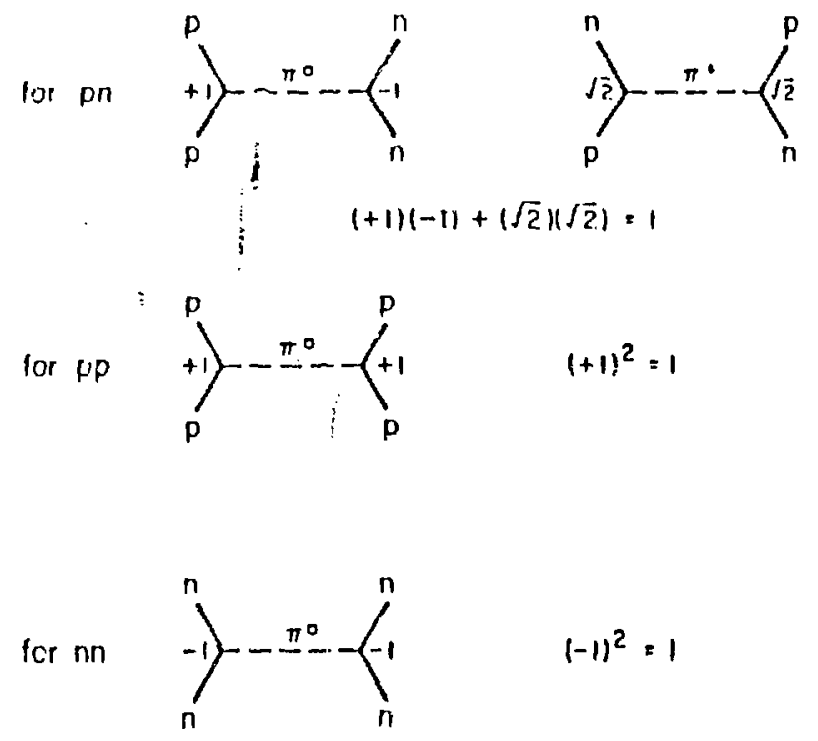

Figure 1.1: Feynman diagrams for one pion exchange nucleon-nucleon potential.

It is convenient to discuss the issue of isospin vjolation in meson theory with refcrence to $\mathrm{OP} \mathrm{EP}$, where the analysis is more straightforward, and subsequently generalize to the terms which appear in the description of the interaction at shorter ranges. The so called 'direct electromagnetic effects', which represent the synme- 
try breaking terms which vanish if the strong interaction is switched off, will not be considered.

Charge independence would require the equality of the masses $\mathrm{m}\left(\pi^{ \pm}\right)$and $\mathrm{m}\left(\pi^{0}\right)$ of the charged and neutral pions and the equality of the couplings $g_{ \pm}$and $g_{0}$ associated with the respective nucleon-pion interaction vertex. It is known that, whereas $\mathrm{m}\left(\pi^{+}\right)$is exactly equal to $\mathrm{m}\left(\pi^{-}\right)$as it is required to satisfy the CP'T theorem (the two charged pions are related by particle-antiparticle conjugation), there is a relatively large difference between $\mathrm{m}\left(\pi^{-}\right)$and $\mathrm{m}\left(\pi^{0}\right)$ :

$$
\frac{\Delta m\left(\pi^{ \pm}\right)}{\langle m(\pi)\rangle}=\frac{4.604 \pm 0.004}{137.27}=3.4 \%
$$

This mass splitting, in the framework of the meson theories, is attributed to electromagnetic renormalization and is considered to be the major source of isospin violation.

An analogous elecromagnetic renormalization of the coupling constants is expected, but it is more difficult to evaluate. However it is expected to be small: the difference between the pseudoscalar coupling constants is estimated to be $\leq$ $1 \% 11$

The other major source of isospin symmetry violation in this approach is the so called 'meson mixing'. Since there are some mesons which have the same spacetime symmetry and the same charge, but different isospin quantum numbers, a isospin violating interaction, like the electromagnetic one, can lead to a mixing between these mesons. This is illustrated by the diagram in Fig. 1.2, which describes the ' $\pi-\eta$ ' and the ' $\rho-\omega$ ' mixing.

The description of the isospin breaking in the shorter range of the interaction becomes more complicated, as the number of possible diagrams which contribute becomes large, (the $\rho-\omega$ mixing is illustrated above), corresponding to a more limited understanding of this range of the interaction in this theoretical framework. In the literature various calculations can be found, based on the corrections due to two pion exchange potential ${ }^{13}$, to the $p-\omega$ mixing ${ }^{14-18}$ and to the difference in 

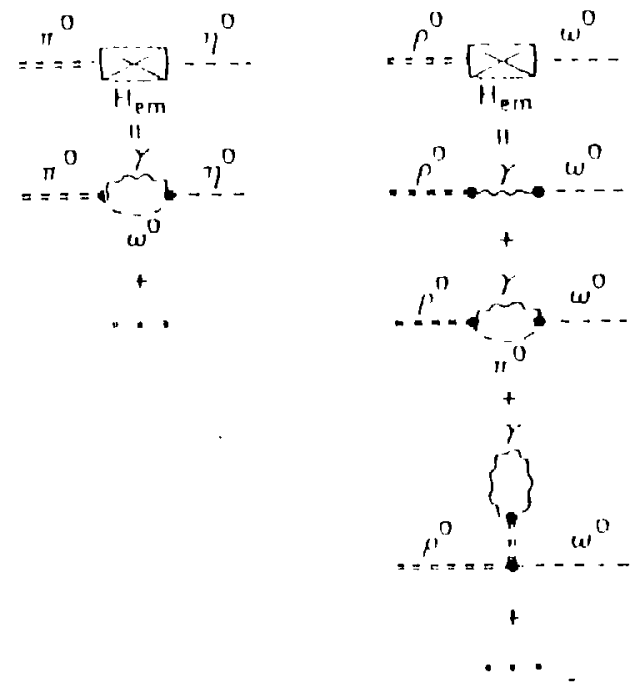

Figure 1.2: Examples of ' $\pi-\eta$ ' and the ' $\rho-\omega$ ' mixing diagrams.

mass between the intermediate isobar excited states ${ }^{19}$. Therefore it appears that a quantitative theoretical treatment of the isospin symmetry violation in this range of the interaction not only 'still escapes us', as Henley admits ${ }^{12}$, but is likely to continue to elude us unless a more fundamental treatment of the interaction is developed. 


\subsection{Isospin in the context of QCD}

It is fair to say that no dramatic change in the role played by isospin symmetry in our understanding of nuclear dynamics took place until new phenomenological models were inspired by quantum chromodynamics (QCD), which is so far the most promising theory of strong interactions. According to QCD, all the hadrons are composite states of quarks. Therefore the quanturn numbers characterizing the hadrons arise as the $a \mathrm{~m}$ of the corresponding ones carried by their elementary constituents.

The isospin quantum number is non-zero for the lightest quarks, up(u) and down (d), which are the 'valence' constituents of the nucleons. In this context, isospin symmetry represents a sub-group of a larger symmetry exhibited by a sector of the QCD Lagrangian $L$, which can be written as:

$$
L^{Q C D}=-\frac{1}{4} F_{\mu \nu}^{a} F^{\mu \nu a}+i \bar{\psi} \gamma^{\mu} D_{\mu} \psi+\Delta L^{Q C D}
$$

where:

$$
F_{\mu \nu}^{a}=\partial_{\mu} A_{\nu}^{a}-\partial_{\nu} A_{\mu}^{a}+g f^{a b c} A_{\mu}^{b} A_{\nu}^{c}
$$

is the Yang-Mills field strength,

$$
\psi=\left(\begin{array}{c}
q_{1} \\
\vdots \\
q_{i} \\
\vdots \\
q_{n}
\end{array}\right)
$$

is the quark vector (the index $i$ runs over flavors), $D_{\mu}$ is the covariant derivative, and

$$
\Delta L^{Q C D}=\bar{\psi} M \psi=\Sigma m_{i} \bar{q}_{i} q_{\mathrm{i}}
$$


The mass term of the Lagrangian, $\triangle L^{Q C D}$, has been singled out because it is the only term which breaks a $S U(N)_{L} \Theta S U(N)_{R}$ symmetry, where $N$ corresponds to the number of quark llavors. This term is actually an approximation of the mass sector of the full 'standard model' of the strong and electroweak interactions, which is valid at energies low with respect to the masses of the $W$ and $Z$ bosons, i.e. $\approx 90 \mathrm{GeV}$. In fact, in this model the fermion masses can not be introduced as free parameters of the theory, because explicit mass terms would break the $S U(2)_{\text {weuk }}$ invariance of the Lagrangian. Their dynamical origin is attributed to the spontaneous symmetry breaking produced by the vacuum expectation value of the lliggs fields. This expectation vaiue, together with the couplings of the fermions to the lliggs particles, are the paraneters which are equivalent to the bare fermion masses. At low energy it is possible to neglect the weak interaction and describe the dynamics of fundamental particles in terms of $L^{Q C D}$ and the analogous Lagrangian density $L^{Q E D}$, describing the elecromagnetic interaction.

The first feature which emerges from this theoretical approach is the 'accidental' nature of isospin symmetry, which is valid, at least approximately, in the hadronic phenomenology. In fact, up and down quarks seem to have no rcason to be singled out with respect to heavier quarks, like the charm and the bottom. Howce $\mathrm{eCD}$ is a theory with a scale, which is set by the phase transition which takes plare upon hadronization. It is widely believed that in this process the vacuum undergoes a phase transition which breaks the chiral symmetry discussed above according to the pattern: $S U(N)_{L} \otimes S U(N)_{R} \longrightarrow S U(N)_{V}$. The order parameter associated with this phase transition, $\Lambda_{C S B},(\approx 1 \mathrm{GeV})^{20}$, sets the mass scale in hadronic phenomenology: the up, down and, to a lesser extent, the strange quarks, which have bare masses of the order of $4,10,140 \mathrm{MeV}$ respectively, are 'light' and, to a first degree of approximation, can be treated as massless. This is the reason why the discussion of the flavor symmetry breaking is generally performed with respect to $S U(3)$ and not $S U(2)$, in other words $m_{\text {s }}$ is also considered $\approx 0$. As a consequence of the phase transition discussed above, 
Table 1.1: Evaluations of ratios between current algebra masses of the light quarks.

\begin{tabular}{|c|c|l|}
\hline$\frac{m_{u}}{m_{d}}$ & $\frac{m_{d}}{m_{g}}$ & Evaluation Method \\
\hline $0.381 \pm 0.13$ & $0.045 \pm 0.011$ & $\begin{array}{l}\text { Chiral perturbation } \\
\text { theory and baryon spectrum }{ }^{21}\end{array}$ \\
$0.47 \pm 0.11$ & $0.042 \pm 0.008$ & $\begin{array}{l}\text { Pseudoscalar meson masses and decay } \\
\text { constants and } \rho-\omega \text { mixing }^{22}\end{array}$ \\
$0.14 \pm 0.07$ & 0.05 & $\eta^{\prime} \longrightarrow 3 \pi^{23}$ \\
$0.41 \pm 0.15$ & $0.044 \pm 0.008$ & $\eta 3 \pi^{24}$ \\
$0.24-0.73$ & $0.039-0.056$ & $\begin{array}{l}\text { Chiral perturbation theory }^{25} \\
(2 \mathrm{nd} \text { order terms } \leq 20 \%)\end{array}$ \\
\hline
\end{tabular}

nine Goldstone bosons arise, corresponding to the generators of the subgroups broken in this process. They are identified with the nonet of lightest pseudoscalar mesons the $\pi$ 's, $\eta$ 's, $\eta$ 's, and K's. In this approximation they should be massless. They acquire a mass because of the breaking of $S U(3)_{V}$, which is generated by the unequal values of $\mathrm{m}_{u}, \mathrm{~m}_{d}$, and $\mathrm{m}_{s}$. The matrix which generates the physical masses of these mesons and their couplings is directly related to these parameters. The empirical values of its elements are the most commonly used method to extract ratios between $\mathrm{m}_{u}, \mathrm{~m}_{d}, \mathrm{~m}_{\boldsymbol{s}}$. The mass spectrum of the pseudoscalar mesons and the branching ratios for some specific decays, like $\eta \rightarrow 3 \pi$, allow the determination of the quantities $r_{+} \equiv\left(m_{u}+m_{d}\right) / m_{\varepsilon}$ and $r_{-} \equiv\left(m_{d}-m_{u}\right) / m_{\varepsilon}$, from which the ratios $r=m_{u} / m_{d}$ and $r^{\prime}=m_{d} / m_{s}$ can be extracted. The information presently ayailable is summarized in Table 1.1.

It can be seen that the general consensus is that at a quark level $S U(2)$ is badly broken. It is interesting to remark that the debate on the current algebra mass ratios for light quarks is still an open subject. Recently ${ }^{25}$ a study of second order contribution in the chiral perturbation theory suggests that, under the hypothesis that the second order contribution to the meson masses is equal to 
$30 \%$, the ratic $m_{u} / m_{d}$ can have any value between 0.0 and 0.8 . Even if $m_{u}$ and $\mathrm{m}_{d}$ are actually very different, the large renormalization which takes place upon hadronization washes out this asymmetry almost completely.

It is interesting to note that this is the first time in which a mechanism that is entirely independent of the electromagnetic effect has been identified for isospin nonconservation. This is the reason why it is generally known as anomalous isospin breaking'. ${ }^{26-27}$ The task of deriving the consequences of the value of the ratio $r=m_{\mathrm{u}} / m_{\mathrm{d}}$ in hadronic phenomenology shares the problems of all the models which use the theory of the fundamental interaction as 'inspiration' for their guesswork. In other words, as we lack the mathematical ability to solve the problem exactly, it is recessary to resort to models which try to incorporate the relevant dynamical features of the underlying theory in an approximation scheme which is calculable.

The simplest approach which can be taken is to introduce the anomalous isospin breaking in the diagrams involving meson $-\cdots$ ixing. $\left(\pi-\eta-\eta^{\prime}, \rho-\omega\right) .{ }^{27}$ These amplitudes were considered also in the meson theories. The latter models derived the couplings phenomenologically from known decays, and therefore obtained essentially correct results. However the new theoretical approuch has the advantage of a more consistent theoretical basis, which allows the recognition of new features which were not previously considered (for instance the importance of $\eta^{\prime}-\pi$ mixing in nucleon-nucleon scattering.) 
1.5 A scale for charge symmetry and charge independence violation.

Even a cursor ${ }^{2}$ and superficial examination of many of the observables of hadronic physics reveals that CS and CI are obeyed to a surprising degree. A scale for $\mathrm{CS}$ and $\mathrm{Cl}$ violations is easily set by the Coulomb interaction, because, as we have already noted, this interaction violates both CS and CI, and it is essentially omnipresent. The strength of the Coulomb interaction compared to the strong hadronic interaction is often stated as $\alpha=1 / 137$, i.e., $\sim 1 \%$. Thus CS and CI violations of this order are to be more or less 'expected'. because of this one reason alone. The 'true' violations, defined as those in the purely hadronic interaction, must come above and beyond the Coulomb interaction induced violations. Let us look at the general experimental evidence.

\subsubsection{Masses of Isospin Multiplets.}

Charge independence implies that masses of the different members of an isospin multiplet (same $T$ or $t$, but different allowed $T_{3}$ or $t_{3}$ ) should be the same, except for Coulomb energy in the case of composite systems.

If we ignore the quark-gluon sub-structure of hadrons for the present, Cl predicts that masses of different charge members of baryon and meson multiplets must be the same. Experimentally this is not found to be the case. $\Lambda$ s Table 1.2 shows, the masses differ.

The difference is generally $<1 \%$, but in one case, $m\left(\pi^{ \pm}\right)-m\left(\pi^{\circ}\right)$, it is as much as $3.3 \%$.

Nuclei are of course composite. Thus the masses within a nuclear isospin multiplet are meant to be not equal but different by the Coulomb energy, calculated in some simple model of nuclear structure. Two approarhes can be taken. $\mathrm{G}_{\text {lie }}$ is to determine the functional form of the Coulomb energy based on general principles and the other is to calculate the absolute value of Coulomb energy. Both approaches have been tried. 
Table 1.2: Masses of diffrent charge members of baryon and meson multiplets

\begin{tabular}{|l|c|l|c|c|}
\hline & $\begin{array}{c}\Delta \mathrm{M} /\langle\mathrm{M}\rangle \\
(\%)\end{array}$ & $\begin{array}{l}\Delta \mathrm{M} \\
(\mathrm{MeV})\end{array}$ & $\begin{array}{c}\Delta \mathrm{M}_{i h}^{*} \\
(\mathrm{MeV})\end{array}$ & $\begin{array}{c}\Delta \mathrm{M}_{t h}^{*} \\
(\mathrm{MeV})\end{array}$ \\
\hline $\mathrm{pn}$ & 0.14 & $-1.239(0)$ & -1.29 & \\
$\Sigma^{+}-\Sigma^{0}$ & 0.26 & $-3.10(14)$ & -1.83 & \\
$\Sigma^{0}-\Sigma^{-}$ & 0.41 & $-4.88(1)$ & -3.45 & \\
$\Xi^{0}-\Xi^{-}$ & 0.49 & $-6.4(6)$ & -4.02 & \\
$\Xi^{* 0}-\Xi^{*-}$ & 0.42 & $-2.90(99)$ & -3.01 & \\
$\pi^{ \pm}-\pi^{0}$ & 3.35 & $+4.604(4)$ & +1.61 & +4.0 \\
$\rho^{ \pm}-\rho^{0}$ & $\approx 0$ & $-0.3(22)$ & +0.94 & +0.7 \\
$\mathrm{~K}^{ \pm}-\mathrm{K}^{0}$ & 0.81 & $-4.01(13)$ & -1.62 & -4.2 \\
$\mathrm{~K}^{*+}-\mathrm{K}^{* 0}$ & 0.29 & $-4.1(6)$ & -1.11 & -1.6 \\
\hline
\end{tabular}

* Bag Model calculation (Ref.28)

** Potential model calculation (Ref.29)

First order perturbation theory ieads to the simple result ${ }^{30}$ that

$$
m\left(T_{3}\right)=a+b T_{3}+c T_{3}^{2}
$$

where $a, b$, and $c$ are constants for an isospin multiplet. No higher order terms are expected if $\mathrm{CI}$ is assumed. Thus the empirically determined presence of a cubic term $d T_{3}^{3}$ would indicate CI violation. Detailed studies of $T=3 / 2$ quartets and $T=2$ quintets $^{31}$ have been done but generally no statistically significant $\mathrm{d}$-terms have been found. Thus, for example, for the $A=37$ quartet the value

$$
d / b=(0.8 \pm 2.1) \times 10^{-3}
$$

which is much smaller than $(\bar{Z} \alpha)^{2}=18 \times 10^{-3}$.

Quantitative calculations of Coulomb energy have been made for nuclei from $A=3$ to $A=41$ within the framework of their known nuclear structure. The simplest of these is of course the ${ }^{3} \mathrm{H}-{ }^{3} \mathrm{He}$ doublet. The experimental beta-decay energy of ${ }^{3} \mathrm{H}$ is $-18.594(8) \mathrm{keV}$, which is equivalent to a difference of $763.745(1 \mathrm{i})$ 
Table 1.3: Coulomb energy shift and correction terms (in MeV) (from Ref. 33).

\begin{tabular}{|l|c|c|c|c|c|c|}
\hline \multirow{2}{*}{ Contribution } & ${ }^{15} \mathrm{O}-{ }^{15} \mathrm{~N}$ & \multicolumn{2}{|c|}{${ }^{17} \mathrm{~F}_{-}{ }^{17} \mathrm{O}$} & ${ }^{39} \mathrm{Ca}^{39}{ }^{39} \mathrm{~K}$ & \multicolumn{2}{c|}{${ }^{41} \mathrm{Sc}^{41} \mathrm{Ca}$} \\
\cline { 2 - 7 } & $1 \mathrm{p}_{1 / 2}^{-1}$ & $1 \mathrm{~d}_{5 / 2}$ & $2 \mathrm{~s}_{1 / 2}$ & $1 \mathrm{~d}_{3 / 2}^{-1}$ & $1 \mathrm{f}_{7 / 2}$ & $2 \mathrm{P}_{3 / 2}$ \\
\hline Direct Coulomb & +3.420 & +3.390 & +3.050 & +7.050 & +6.850 & +6.550 \\
Exchange Coulomb & -0.210 & -0.190 & -0.150 & -0.300 & -0.250 & -0.210 \\
C.M. motion & -0.070 & -0.070 & -0.070 & -0.040 & -0.040 & -0.040 \\
Proton size & +0.065 & +0.095 & +0.085 & +0.090 & +0.110 & +0.100 \\
Neutron size & -0.035 & -0.035 & -0.035 & -0.040 & -0.040 & -0.040 \\
Magnetic interaction & +0.100 & -0.060 & +0.025 & +0.130 & -0.090 & -0.020 \\
Vacuum polarization & +0.020 & +0.020 & +0.020 & +0.040 & +0.040 & +0.040 \\
p-n mass difference & +0.025 & +0.035 & +0.035 & +0.025 & +0.035 & +0.035 \\
short range & +0.065 & +0.045 & +0.050 & +0.125 & +0.075 & +0.105 \\
correlations & & & & & & \\
\hline Total(calculated) & +3.38 & +3.23 & +3.01 & +7.08 & +6.69 & +6.52 \\
Experimental & +3.54 & +3.54 & +3.17 & +7.30 & +7.28 & +7.05 \\
Discrepancy & +0.16 & +0.31 & +0.16 & +0.22 & +0.59 & +0.53 \\
\hline
\end{tabular}

keV between the binding energies of ${ }^{3} \mathrm{H}$ and ${ }^{3} \mathrm{He}$. This s about $10 \%$ of the total binding energy of either nucleus. The best calculations are only able to account for $683 \pm 29 \mathrm{keV}$ of this difference as due to the Coulomb energy of ${ }^{3} \mathrm{He}$. The unexplained $81 \pm 29 \mathrm{keV}$, i.e., $1 \%$ of the binding energy is often quoted as evidence for CS breaking and or three-body forces.

For heavier nuclei detailed Coulomb energy calculations have been done by many authors. The first of these revealed ${ }^{32}$ that the $c>1$ culated Coulomb energies always fall short of the experimental ones. This is the well known Nolen-Schiffer anomaly. A detailed review of the subject has been made by Schlomo ${ }^{33}$. Table 1.3, taken from him, summarizes the present situation. Calculations still fall short of the experimental Coulomb energies, by about $7 \%$. We will not go here into the speculations about the possible causes. 


\subsubsection{Isospin Mixing}

Forces which violate isospin conservation mix states with different isospins. At the elementary particle level this should produce mixing between $\pi^{\circ}(t=1$, $m=135 \mathrm{MeV})$ and $\eta^{\circ}(t=0, m=549 \mathrm{MeV})$, and between $\rho^{\circ}(t=1, m=770 \mathrm{MeV})$ and $\omega^{\circ}(t=0, m=783 \mathrm{Mev})$. The $\pi^{\circ}-\eta^{\circ}$ mixing is expected to be small because of the large difference between their masses. On the contrary, the $\rho^{\circ}-\omega^{\circ}$ mixing is known to be large. The branching ratio for the decay $\omega^{\circ} \rightarrow \rho^{\circ}\left(\pi^{+} \pi^{-}\right)=1.4 \pm 0.2 \%$.

At the nuclear level isospin mixing is well known. Onc way of measuring isospin impurity is to measure isospin forbidden Fermi $\beta^{ \pm}$decays. $\Lambda$ s Fig. 1.3 (from Rer. 34) shows, the matrix elements for these decays are $\sim 10 \mathrm{keV}$. Since typical separation between $T$ (upper) and $T$ (lower) states are about $5 \mathrm{MeV}$, a measure of the isospin violation is $\sim 10 \mathrm{keV} / 5 \mathrm{MeV} \approx 0.2 \%$.

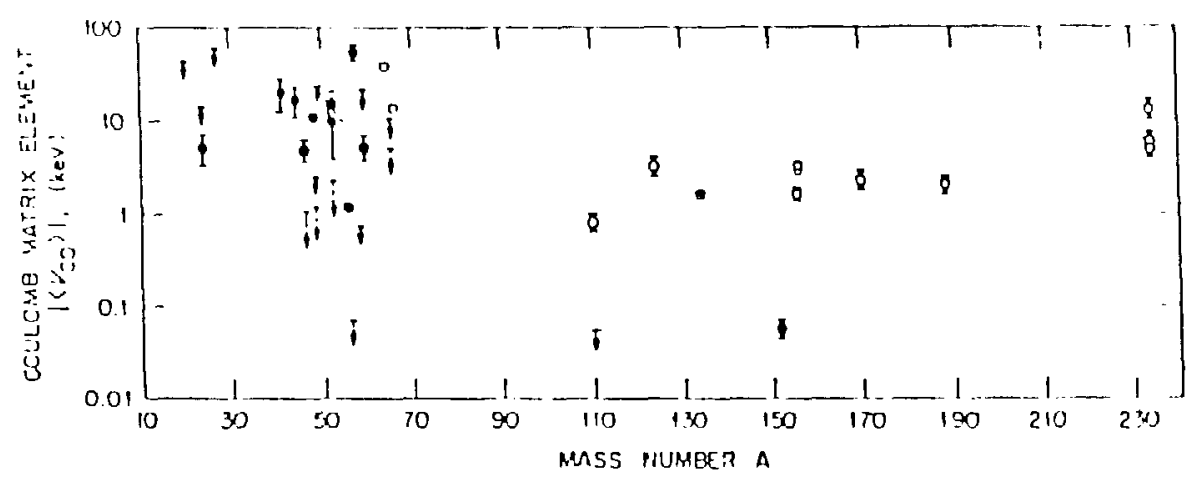

Figure 1.3: Isospin mixing matrix elements inferred from isospin forbidden Formi $\beta^{ \pm}$decays.(from Ref. 34 )

In favourable cases large isospin mixing can result. Almost pure neutron and almost pure proton states (which correspond to 50-50 isospin admixtures) have becn observed. Sonc of the well known cases are illustrated in Table 1.4 due to Adelberger. ${ }^{34}$ The model calculation referred to in this table is one in which the whole mixing is cluc to single particle Coulomb energy dilferences. It ss somewhat surprising, but gratifying that even such a simple calculation explains the observed 
Table 1.4: Isospin mixed doublets (from Ref. 34).

\begin{tabular}{|c|c|c|c|c|}
\hline Nucleu & & $\mathrm{E}_{x}(\mathrm{MeV})$ & $-\mathrm{H}_{\exp }^{\mathrm{c}}(\mathrm{KeV})$ & $-\mathrm{H}_{\text {model }}^{\mathrm{c}}(\mathrm{KeV})$ \\
\hline${ }^{8} \mathrm{Be}$ & $2^{+}$ & 16.6 & 145 & 88 \\
\hline & & $\begin{array}{l}16.9 \\
127\end{array}$ & & \\
\hline${ }^{12} \mathrm{C}$ & $1^{+}$ & 15.1 & $130 \pm 26$ & 125 \\
\hline${ }^{14} \mathrm{~N}$ & $0^{-}$ & $\left.\begin{array}{l}4.9 \\
8.8\end{array}\right)$ & $395 \pm 74^{(a)}$ & 362 \\
\hline${ }^{14} \mathrm{~N}$ & $3^{-}$ & $\left\{\begin{array}{l}5.8 \\
8.9\end{array}\right.$ & $\leq 103$ & 154 \\
\hline${ }^{16} \mathrm{O}$ & $1^{-}$ & $\begin{array}{c}7.1 \\
13.1\end{array}$ & $591 \pm 54$ & 494 \\
\hline${ }^{16} \mathrm{O}$ & $2^{-}$ & $\left.\begin{array}{l}12.5 \\
13.0\end{array}\right\}$ & $\geq 155 \pm 30$ & 163 \\
\hline${ }^{24} \mathrm{Mg}$ & $4^{+}$ & $\left\{\begin{array}{l}8.4 \\
9.5\end{array}\right\}$ & $106 \pm 40$ & 71 \\
\hline
\end{tabular}

a Experiment does not fix the sign of $\mathrm{H}^{c}$ but it is expected to be negative.

mixing essentially perfectly.

Now we wish to discuss results of experiments designed for the specific purpose of measuring $\mathrm{CI}$ and CS breaking effects. For the purposes of our presentation, we will first consider the weaker principle, CS, and then CI.

There are several excellent reviews of CS and CI breaking. ${ }^{10-12,35}$ They contain detailed descriptions of the experimental data. We will therefore not repeat these descriptions. Instead we will only describe the latest experiments and their impact on our understanding of CS and CI breaking. 


\subsection{Experimental evidence for charge symmetry.}

We will begin by reviewing the latest experiments designed with the specific purpose of testing the weaker principle, namely charge symmetry.

\subsubsection{Nucleon - Nucleon scattering.}

At low energies nucleon-nucleon scattering takes place almost entirely in the $\ell=0$ partial wave. One can therefore compare the $n n, p p$, and $n p$ scatterings in the $\mathrm{T}=1,{ }^{1} \mathrm{~S}_{0}$ state. This comparison is usually done witl'm the framework of the effective range theory. ${ }^{36}$ Accordingly, the phase shift $\delta_{0}\left(\sigma=4 \pi / \mathrm{k}^{2} \sin ^{2} \delta_{0}\right)$ is given by

$$
\mathrm{k} \cot \delta_{0}=\left(-\frac{1}{\mathrm{a}} \mathrm{j}+\frac{1}{2} \mathrm{rk}^{2}\right.
$$

where $\mathrm{k}$ is the momentum in the center of mass, ' $a$ ' is the scatiering length, and ' $\mathrm{r}$ ' is the effective range. Because of the near-binding of the ${ }^{1} \mathrm{~S}_{0}$ state, $\delta_{0}$ is very close to $90^{\circ}$, and a very small change in the nuclear force results in a very large change in the scattering length (a $1 \%$ change in the potential produces $\sim 3 \mathrm{fm}$, or $\sim 18 \%$ change in ' $a$ '). Thus the effect of CS and CI breaking is greatly magnified.

in table 1.5 we present the latest results for low-energy nucleon-nucleon scattering. The pp scattering data ${ }^{37,38}$ which was of superb quality already in 1966 , re-

Table 1.5: Low energy nucleon-nucleon scattering parameters:effective leng ths and effective ranges

\begin{tabular}{|c|c|c|}
\hline & $\mathrm{a}(\mathrm{fm})$ & $\mathrm{r}(\mathrm{fm})$ \\
\hline $\mathrm{pp}^{39}$ & $-7.828 \pm 0.008$ & $2.80 \pm 0.03$ \\
$\mathrm{pp}($ corrected for electromagnetic effects) & $-17.2 \pm 2.0$ & $2.84 \pm 0.16$ \\
$\mathrm{np}^{36}$ & $-23.715 \pm 0.0 \mathrm{i} 5$ & $2.73 \pm 0.03$ \\
$\mathrm{nn}^{43}$ & $-18.6 \pm 0.5$ & $2.83 \pm 0.11$ \\
\hline
\end{tabular}

mains unchanged. There is, however, an improved evaluation of the parameters ${ }^{39}$. These are: $a_{p p}=-7.828(8) \mathrm{fm}, \mathrm{r}_{\mathrm{pp}}=2.80(2) \mathrm{fm}$. The main problem concerns Coulomb and other electromagnetic corrections which must be applied to these. 
Sauer and Walliser ${ }^{40}$ showed that these corrections depend sensitively on the unknown short-range nuclear force, and reached quite pessimistic conclusions about the uncertainty in these corrections. Even if we temper these conclusions with the criteria of reasonableness ${ }^{41}$ and of keeping relativistic effects small ${ }^{42}$, the model dependence of these corrections introduces an error of $\sim 2.0 \mathrm{fm}$ in $\mathrm{a}_{\mathrm{pp}}^{\mathrm{c}}$. Thus the best results one can quote are: $a_{p p}^{c}=-17.2 \pm 2.0 \mathrm{fm}$, and $r_{p p}^{c}=2.84 \pm 0.03 \mathrm{fm}$.

The best estimate of $\mathrm{nn}$ scattering length to date comes from the absorption of stopped pions on deuterium $\pi^{-} \mathrm{d} \rightarrow \mathrm{nn} \gamma$. In this reaction one takes advantage of the fact that in the final state the two neutrons are the only strongly interacting particles. Near the maximum of $\gamma$-ray energy, these neutrons have very small relative energy, and one can deduce $a_{n n}$ and $r_{n n}$ from their final state interaction. By doing a high-precision, high statistics experiment Gibioud et $\mathrm{a} \mathrm{l}^{43}$ have been able to obtain $a_{n n}=-18.6 \pm 0.5 \mathrm{fm}$, and $r_{n n}=2.83 \pm 0.11 \mathrm{fm}$. The analysis of these data does not suffer from the problems mentioned with the pp data.

There is report of a direct nn scattering experiment which is under preparation by a Los Alamos-Oak Ridge collaboration ${ }^{44}$ in which an attempt will be made to measure nn scattering cross section by colliding two beams of $14 \mathrm{MeV}$ neutrons from two simultaneously detonated fusion-fission devices (euphemism for bombs). The beams are meant to collide at $3.8^{\circ}$ so that a c.m. collision energy of $38 \mathrm{keV}$ is realized for the $14 \mathrm{MeV}$ neutrons. It is claimed that a $10 \%$ measurement of cross sections will determine $a_{n n}$ to better than $3 \%$ or $\pm 0.5 \mathrm{fm}$. This would certainly be great. It is reported that on April 6, 1986, the first stage of this experiment, i.e. feasibility test for simultaneous detonation, was made. We look forward to the definitive experiment in 1988.

From the best present values of $a_{n n}$ and $a_{p p}^{c}$ we obtain

$$
\Delta \mathrm{a}=\mathrm{a}_{\mathrm{nn}}-\mathrm{a}_{\mathrm{pp}}^{\mathrm{c}}=-1.4 \pm 2.0 \mathrm{fm}
$$

The difference is thus not significant. 


\subsubsection{Pion - Deuteron Total Cross Sections.}

Pedroni et al..$^{45}$ have made high precision measurement of $\pi^{-} \mathrm{d}$ and $\pi^{+} \mathrm{d}$ total cross sections in the pion energy range $70-370 \mathrm{MeV}$. After correcting their results for the direct effects of the Coulomb potential, they find energy dependent differences, ranging from $-6 \%$ to $+2 \%$, between $\pi^{-} \mathrm{d}$ and $\pi^{+} \mathrm{d}$ cross sections (sce Fig. 1.4).

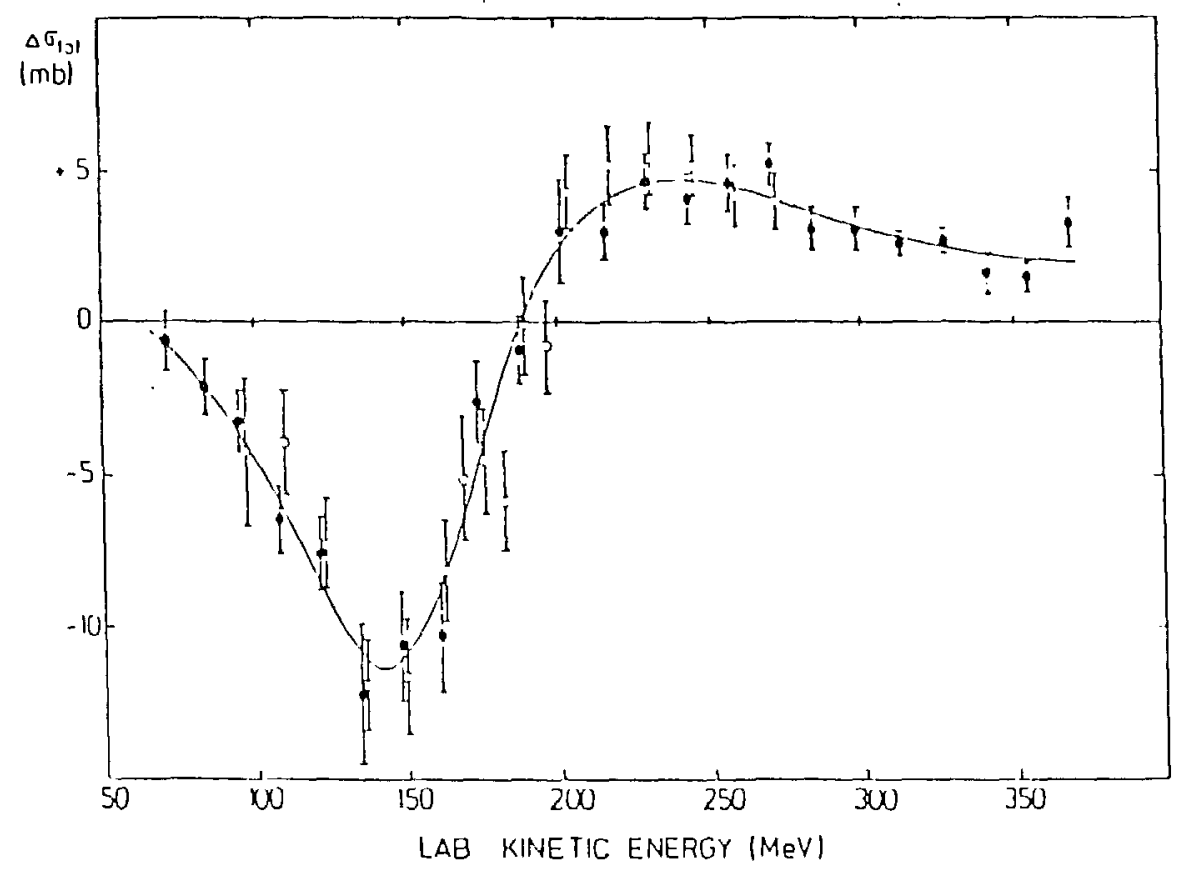

Figure 1.4: Difference in $\sigma_{t}\left(\pi^{-} d\right)$ and $\sigma_{t}\left(\pi^{+} d\right)$.(from Ref. 45)

They interpret these differences as being due to charge symmetry violation and parameterize them in terms of mass difference, $m\left(\Delta^{0}\right)-m\left(\Delta^{++}\right)=2.7 \pm 0.3$ $\mathrm{MeV}$, or $\sim 0.2 \%$, and width difference, $\Gamma\left(\Delta^{0}\right)-\Gamma\left(\Delta^{++}\right)=6.6 \pm 1.0 \mathrm{MeV}$, or $6.0 \%$. 


\subsubsection{Pion - Deuteron Elastic Scattering.}

After removal of electromagnetic effects $\pi^{+} \mathrm{d}$ and $\pi^{-} \mathrm{d}$ elastic scattering differential cross sections should be equal if charge symmetry holds. Balestri et al. ${ }^{46}$ have measured these cross sections at $\mathrm{T}(\pi)=65 \mathrm{MeV}$, and Masterson et al. have measured them at $\mathrm{T}(\pi)=142 \mathrm{MeV}^{47}$ and $256 \mathrm{MeV} .{ }^{48}$ Balestri et al. found statistically significant and rather large asymmetries, $\Lambda(\theta)=\sigma\left(\pi^{-}\right)-\sigma\left(\pi^{+}\right) /\left(\sigma\left(\pi^{-}\right)+\sigma\left(\pi^{+}\right)\right)$ in their $65 \mathrm{MeV}$ data. On the other hand, Masterson et al. found angle dependent asymmetries in the range $\mathrm{A}=(2.5 \pm 2.5) \%$ at $142 \mathrm{MeV}$ and $\mathrm{A}=(0 \pm 2) \%$ at $256 \mathrm{MeV}$. All the observed effects appear to be quite satisfactorily accounted for by external Coulomb corrections ${ }^{49}$. (see Fig. 1.5)
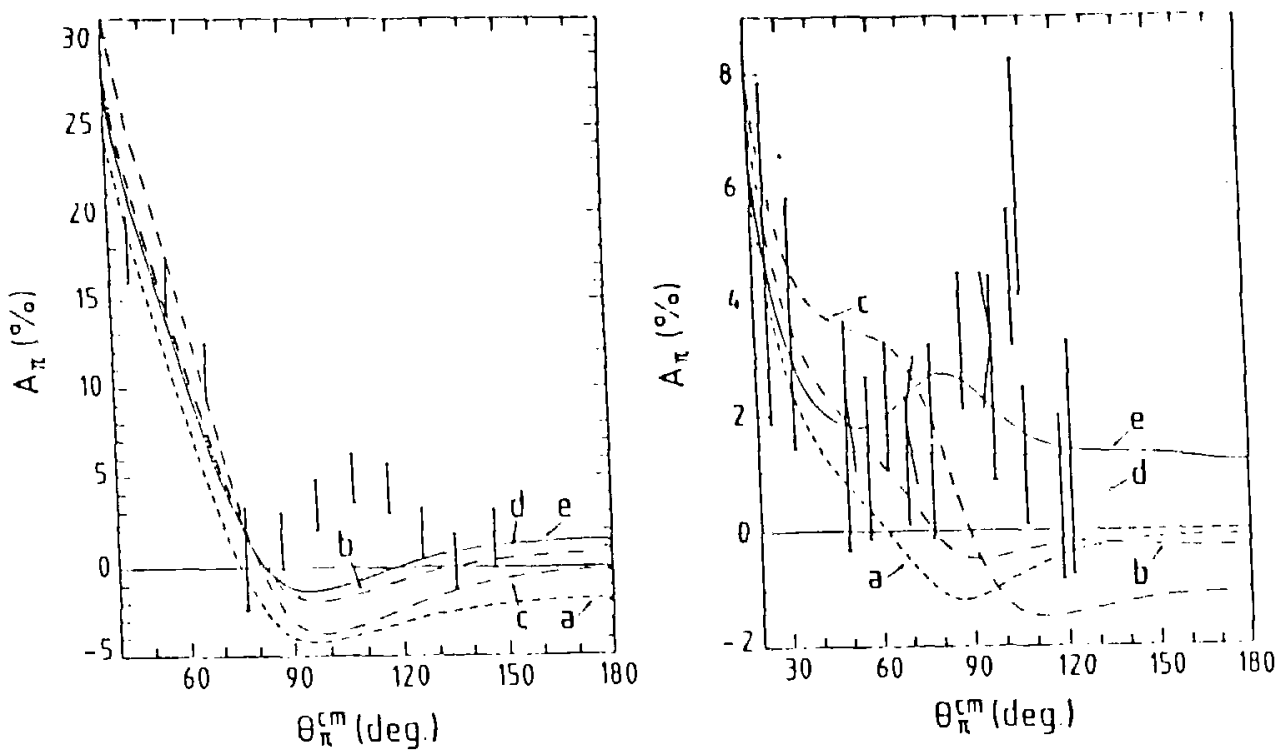

Figure 1.5: Asymmetry $\mathbf{A}(\theta)$ in $\%$ for $\pi^{+}, \pi^{-}$elastic scattering cross sections (from Ref. 49). The figure on left is for $\mathrm{T}(\pi)=65 \mathrm{MeV}$, the one on right is for $\mathrm{T}(\pi)=142$ $\mathrm{MeV}$. 


\subsection{4 $\pi-{ }^{3} H$ and $\pi-{ }^{3}$ He Scattering.}

Charge symmetry requires that the elastic scattering ratios

$$
r_{1}=\frac{\mathrm{d} \sigma\left(\pi^{+}+{ }^{3} \mathrm{II}\right)}{\mathrm{d} \sigma\left(\pi^{-}+{ }^{3} \mathrm{He}\right)}
$$

and

$$
\Gamma_{2}=\frac{\mathrm{d} \sigma\left(\pi^{+}+{ }^{3} \mathrm{He}\right)}{\mathrm{d} \sigma\left(\pi^{-}+{ }^{3} \mathrm{H}\right)}
$$

should be separately equal to unity at all angles. Nefkens et al. ${ }^{50}$ have noted that the super-ratio, $R=r_{1} / r_{2}$, which should also be unity, has the great advantage that for its determination no absolute cross section normalizations are required and target and beam characteristics mostly cancel out. In their first measurement at $\mathrm{T}(\pi)=180 \mathrm{MeV}$, Nefkens et al. found that the super ratio varied with angie and reached a maximum of $R=1.31 \pm 0.09$ at $65^{\circ}$ (see Fig. 1.6). This caused a flurry of excitement. Kim" ${ }^{61}$ rushed in to explain the observations as "manifestation of multiquark compound resonances in interacting hadronic systems", and Barshay and Sehgal ${ }^{52}$ explained the observations in terms of short-range correlations in the three nucleon system, which are distorted in going from ${ }^{3} \mathrm{H}$ to ${ }^{3} \mathrm{He}$. In the meanwhile, Nefkens et al. have done a more extensive series of measurements ${ }^{53}$ in which they have measured the super ratio at five angles $40^{\circ}, 60^{\circ}, 80^{\circ}, 90^{\circ}$, and $110^{\circ}$ at $\mathrm{T}(\pi)=142,180$, and $220 \mathrm{MeV}$. The average of all measurements is found to be $R=1.06(5)$ at $220 \mathrm{MeV}, \mathrm{R}=1.09(4)$ at $142 \mathrm{MeV}$, and $\mathrm{R}=1.10(5)$ at $180 \mathrm{MeV}$. In the $40^{\circ}-80^{\circ}$ interval not a single value larger than $1.11(5)$ is found. At this level it appears entirely likely that external Coulomb effects will explain the observations. 


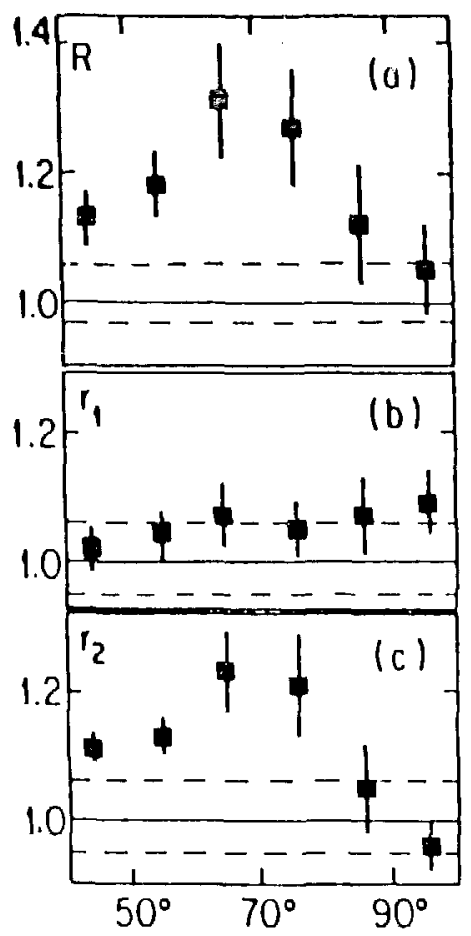

Figure 1.6: Ratios for $\pi-^{3} \mathrm{H}\left({ }^{3} \mathrm{He}\right)$ elastic scattering. $r_{1}=d \sigma\left(\pi^{+}+{ }^{3} \mathrm{H}\right) / d \sigma\left(\pi^{-}+{ }^{3} \mathrm{He}\right)$, $r_{2}=d \sigma\left(\pi^{+}+{ }^{3}\right.$ Ile) $/ d \sigma\left(\pi^{-}+{ }^{3} \mathrm{II}\right), \mathrm{R}=r_{1} / r_{2}$. (from Ref. 50)

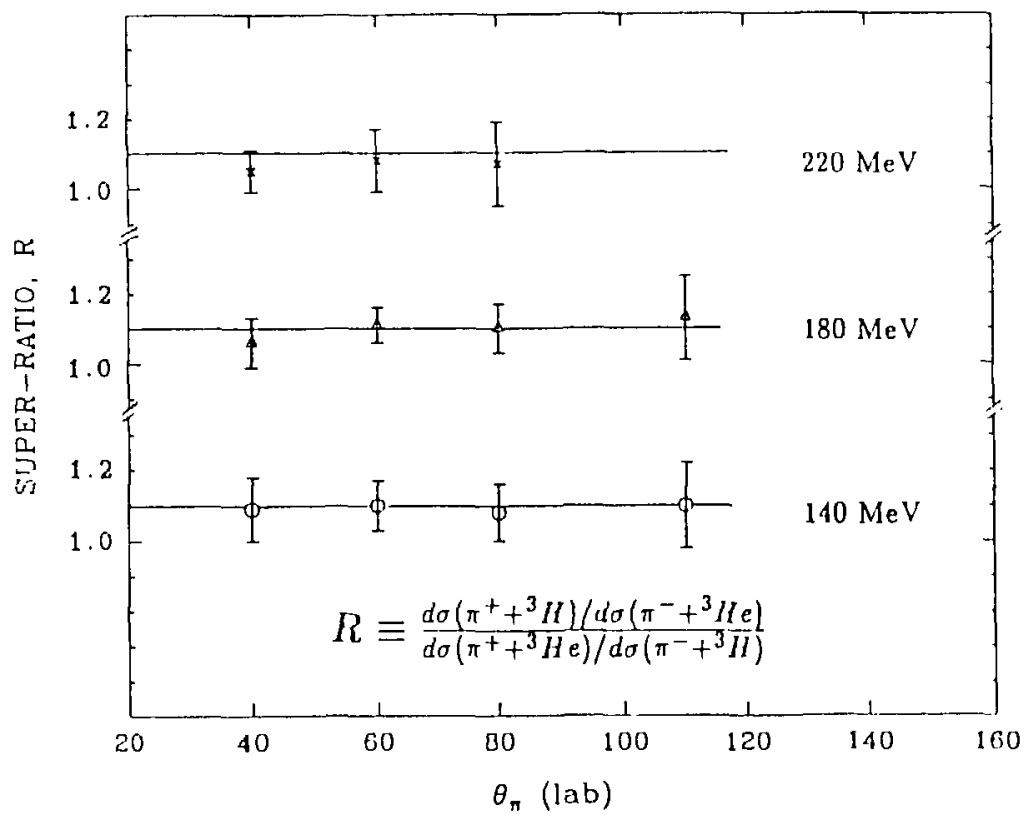

Figure 1.7: Most recent results for the super-ratio IR defined in Fig. 1.6 (from Ref. 53) 


\subsection{5 $\pi-{ }^{4}$ Ine Siattering.}

Charge symmetry requires that $\pi^{+}$and $\pi^{-}$elastic scattering cross sections for ${ }^{4}$ lle should be identical. Recently, Khankhasayev et al. ${ }^{54}$ have done simultaneous phase shift analysis of the available data for $\pi^{ \pm}$scattering from ${ }^{4}$ Ile at $\mathrm{T}(\pi)=24 \mathrm{MeV}, 5 \mathrm{I}$ $\mathrm{MeV}$, and $75 \mathrm{MeV}$. They obtain "quite a good description of the angular depen.. dence of the asymuetry parameter $\Lambda(\theta)$ by taking account of extermal coulonib corrections". (see Pig. 1.8) They find no signilicant charge symmetry breaking ellects.

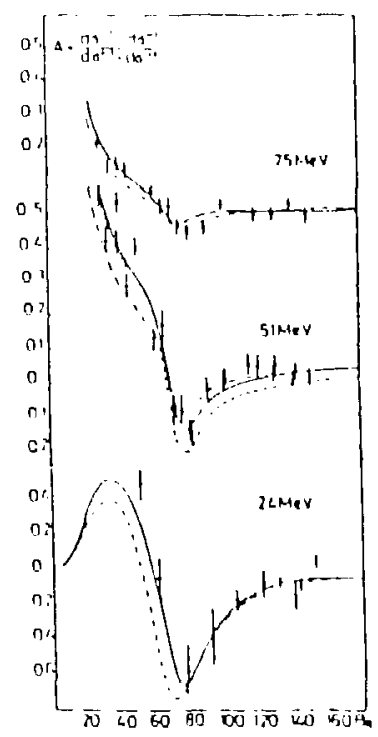

Figure 1.8: Asymmetry $\Lambda(0)$ for $\pi^{ \pm}$elastic scattering from ${ }^{4}$ Ile (from Ref. 51).

At LAMDF experiments are currently in progress to make precision measurements of $\pi^{ \pm}$Ile elastic scattering in the resonance region. ${ }^{55}$ 


\subsubsection{The Reaction $\mathrm{d}+\mathrm{d} \rightarrow{ }^{4}$ ine $+\pi^{0}$.}

Obviously this reaction is forbidden by isospin conservation. However, because only self conjugate 'particles' are involved on both sides it only tests charge symmetry, and not charge independence. This is a difficult experiment, but much progress has been made recently. At Saclay Stetz et al. ${ }^{66}$ have been able to establish upper limits of $\mathrm{d} \sigma / \mathrm{d} \Omega<5 \mathrm{pb} / \mathrm{sr}$ (at ${ }^{\prime} T_{d}=1350 \mathrm{MeV}, 0_{c m}=77^{\circ}$ ) and $\mathrm{d} \sigma / \mathrm{d} \Omega$ $<0.8 \mathrm{pb} / \mathrm{sr}\left(\right.$ at $\left.\mathrm{T}_{d}=800 \mathrm{MeV}, \theta_{c m}=100^{\circ}\right)$. These limits are $142 \%$ and $17 \%$ respectively of the corresponding cross sections for the isospin allowed reaction $\mathrm{d}+\mathrm{d} \rightarrow \alpha+\gamma$. Thus although we have come a long way from the $1100 \mathrm{pb} / \mathrm{sr}$ limit established by Akimov et al..$^{57}$ in 1962, we are still far from the level of sensitivity required to see charge symmetry breaking effects. As a matter of fact Cheung ${ }^{58}$ has predicted cross sections of the order of $0.03 \mathrm{pb} / \mathrm{sr}$ at $\mathrm{T}_{\mathrm{d}}=700 \mathrm{MeV}, \theta=0$. Coon and Precdom ${ }^{59}$ predict a forward differential cross section of the order of $0.12 \mathrm{pb} / \mathrm{sr}$ at $\mathrm{T}_{\mathrm{d}}=1.95 \mathrm{GeV}$.

\subsubsection{The Reaction $\mathrm{np} \rightarrow \mathrm{d} \pi^{\circ}$.}

The differential cross sections for the $\mathrm{T}=1 \rightarrow \mathrm{T}=1$ reaction $\mathrm{pp} \rightarrow \mathrm{d} \pi^{+}$are symmetric about $\theta_{(\text {c.m. }}=90^{\circ}$. If isospin is conserved, the reaction $n p \rightarrow \mathrm{d} \pi^{\circ}$ can only proceed through the $\mathrm{T}=1 \rightarrow \mathrm{T}=1$ channel and its cross sections must also be symmetric about $90^{\circ}$. Departures from syinmetry about $90^{\circ}$ therefore provide a measure of isospin symmetry breaking. The latest experiment testing the forwardbackward symmetry of $\mathrm{np} \rightarrow \mathrm{d} \pi^{\circ}$ cross sections was done by Hollas et al. ${ }^{60}$ who found the asymmetry parameter $\mathrm{A}=-0.15 \pm 0.50$ at $\mathrm{T}(n)=795 \mathrm{MeV}$. Cheung at al. ${ }^{61}$ have predicted $A=-0.11$ at this energy, primarily due ${ }^{r} \pi^{n}-\eta$ mixing. It would be very useful indeed if the errors on the experimental measurement could be reduced. 


\subsubsection{Neutron-Proton Elastic Scattering.}

As early as 1956, Wolfenstein ${ }^{62}$ noted that in $n-p$ elastic scattering the polarization of the two outgoing particles should be equal:

$$
P_{n}(\theta)=P_{p}(\pi-\theta)
$$

This follows from parity conservation and the generalized Pauli principle or the overall asymmetry of the space-spin-isospin wave function. This implies that only like spin states are connected in the initial and final states (i.e., singlet to singlet, triplet to triplet), i.e., the spin dependent part of the transition operator must be even under particle exchange. This gives equal polarization for $\mathbf{n}$ and p. The same relation holds for analyzing power if polarized beams or targets are used. For example,

$$
\Delta A=A(\vec{n} p, \theta)-A(n \vec{p}, \theta)=0
$$

If charge symmetry is broken, the triplet-singlet transition amplitude $f_{t a}$ is non-zero and leads to non-zero $\Delta A$. As a matier of fact $\Delta A$ is direcily proportional to $f_{t a}$. The ideal experiment of this type would consist of scattering polarized neutrons from a polarized proton target. A single experiment could provide $\Delta A$ by forming the two analyzing powers, $A(n \vec{p})$ by summing over all the neutron spin directions and $A(\vec{n} p)$ by summing over all the proton spin directions.

Two different experiments have been performed recently. At TRIUMF Abegg et $a .^{63}$ have made a precision measurement of the difference between the angles at which $A(n \vec{p})$ and $A(\vec{p} n)$ have zero value for $\mathrm{T}_{n}=477 \mathrm{MeV}$. The measured difference

$$
\Delta \theta=-0.13^{\circ} \pm 0.07^{\circ}\left( \pm 0.03^{\circ}\right)
$$

is equivalent to

$$
\Delta A=0.0034 \pm 0.0017 \pm(0.0008)
$$

at that angle. This compares well with the theoretical result of 


$$
\Delta A=+0.0054 \pm 0.0040
$$

due to Miller, Thomas and Williams ${ }^{64}$. In this calculation charge symmetry breaking terms due to one photon exchange, n-p mass difference affecting $\pi, \rho$, and $2 \pi$ exchanges, $\rho-\omega$ mixing, and effects of the up-down quark masses are included. We note that an earlier prediction for $\Delta A$ gave a negative result $(\Delta A=-.0035)$ due to an error in the sign of the one pion exchange contribution.

The Indiana experiment ${ }^{65}$, which is still under preparation, differs from the TRIUMF experiment in several ways. The experiment will be done at $T_{n}=200$ $\mathrm{MeV}$ with beam and target simultaneously polarized. In this case the left-right asymmetries will be measured at a number of angles $\left(75^{\circ} \leq \theta_{c m} \leq 120^{\circ}\right)$. They should be zero at all angles when the entrance channel neutron and proton spin projections are opposite. It is claimed that the sensitivity to $\Delta A$ will be at the level of 0.001 . 


\subsection{Charge Independence}

As mentioned earlier, charge independence is the stronger syminetry. However, compared to charge symmetry breaking, many fewer tests of charge independence breaking have been made.

\subsubsection{Low Energy np Scattering}

Together with the nn and pp scattering lengths, a measurement of the np scattering provides one of the best tests of charge independence. As listed in Table 1.6, the np singlet scattering length is $a_{n p}=-23.72 \pm 0.02 \mathrm{fm}$. This determination does not suffer from the uncertainties associated with $a_{n n}$ and $a_{p p}$, and leads to

$$
\Delta a=a_{n p}-\left\langle a_{n m}, a_{p p}\right\rangle=(-23.72 \pm .02)-(-18.3 \pm 0.5)=-5.4 \pm 0.5 \mathrm{fm}
$$

This large difference, amounting to $26 \pm 3 \%$, is the basis of the statement that $\mathrm{CI}$ is quite evidently broken. We must, of course, remember that this charge independence breaking due type II forces amounts to

$$
\left|\bar{V}_{n p}\right|-\left|\bar{V}_{n n}\right| /\left\langle\left|\bar{V}_{n p}\right|+\left|\bar{V}_{n n}\right|\right\rangle / 2=(2.1 \div 0.5) \%
$$

We should also note that one pion exchange alone provides a mechanism for $V_{n p}>$ $V_{n n}$ due to $\pi^{ \pm}, \pi^{\circ}$ mass difference $\Delta m$

$$
V_{n p}=V_{n n}\left[-1+2\left(\frac{m^{ \pm}}{m_{r}}\right)^{2} \exp (-\Delta m \cdot r)\right]
$$

This contributes about half of the observed difference in scattering lengths. Recently Cheung and Machleidt ${ }^{\beta \theta}$ have considered $\Delta a$ within the framework of a consistent meson model of NN interactions. They are able to account for essentially all of the observed difference $\Delta \mathrm{a}$ (see Table 1.7).

It should be noted that the s-wave $(l=0)$ scattering lengths do not permit any insight into Henley's type IV charge independence breaking forces. For these we need to go to a polarization observables. 
Table 1.6: Theoretical resolution of the contributions to $\Delta a=a_{n p}-\left\langle a_{n n}, a_{p p}\right\rangle$ (from ref.66).

\begin{tabular}{|l|r|}
\hline Potential & $\delta a(\mathrm{fm})$ \\
\hline OPEP & 1.80 \\
TPEP $(\mathrm{NN})$ & 0.18 \\
TPEP $(\mathrm{N} \Delta)$ & 3.25 \\
TPEP $(\Delta \Delta)$ & -2.58 \\
$\pi \rho$-exchange $(\mathrm{NN})$ & -0.39 \\
$\pi \sigma, \pi \omega$-exchanges & 1.18 \\
\hline Total & 4.44 \\
\hline
\end{tabular}

\subsubsection{Pion-nucleon total cross section.}

If we decompose pion-nucleon total cross sections into cross sections in pure isospin states, as is well known:

$$
\begin{gathered}
\sigma\left(\pi^{-} \mathrm{p}\right)=\frac{1}{3} \sigma_{3 / 2}+\frac{2}{3} \sigma_{1 / 2} \\
\sigma\left(\pi^{+} \mathrm{p}\right)=\sigma_{3 / 2}
\end{gathered}
$$

It follows therefore that according to charge independence:

$$
3 \sigma\left(\pi^{-} \mathrm{p}\right)-\sigma\left(\pi^{+} \mathrm{p}\right)=2 \sigma_{1 / 2}
$$

$\sigma_{1 / 2}$ is of course not zero, but at the peak of the $(3,3)$ resonance its contribution is very small. In any case, the quantity $\Delta \sigma / \sigma \equiv\left[3 \sigma\left(\pi^{-} p\right)-\sigma\left(\pi^{+} p\right) / \sigma\left(\pi^{+} p\right)\right]$ evaluated at the peak of the $(3,3)$ resonance establishes the upper limit of charge independence violation. As Fig. 1.9 shows this upper limit is $\approx 3 \%$. Actually $\sigma_{1 / 2}$ is rather well known from phase shifts analyses, and it can be shown that the CI violation implied by the data is considerably smaller than the upper limit derived above. 


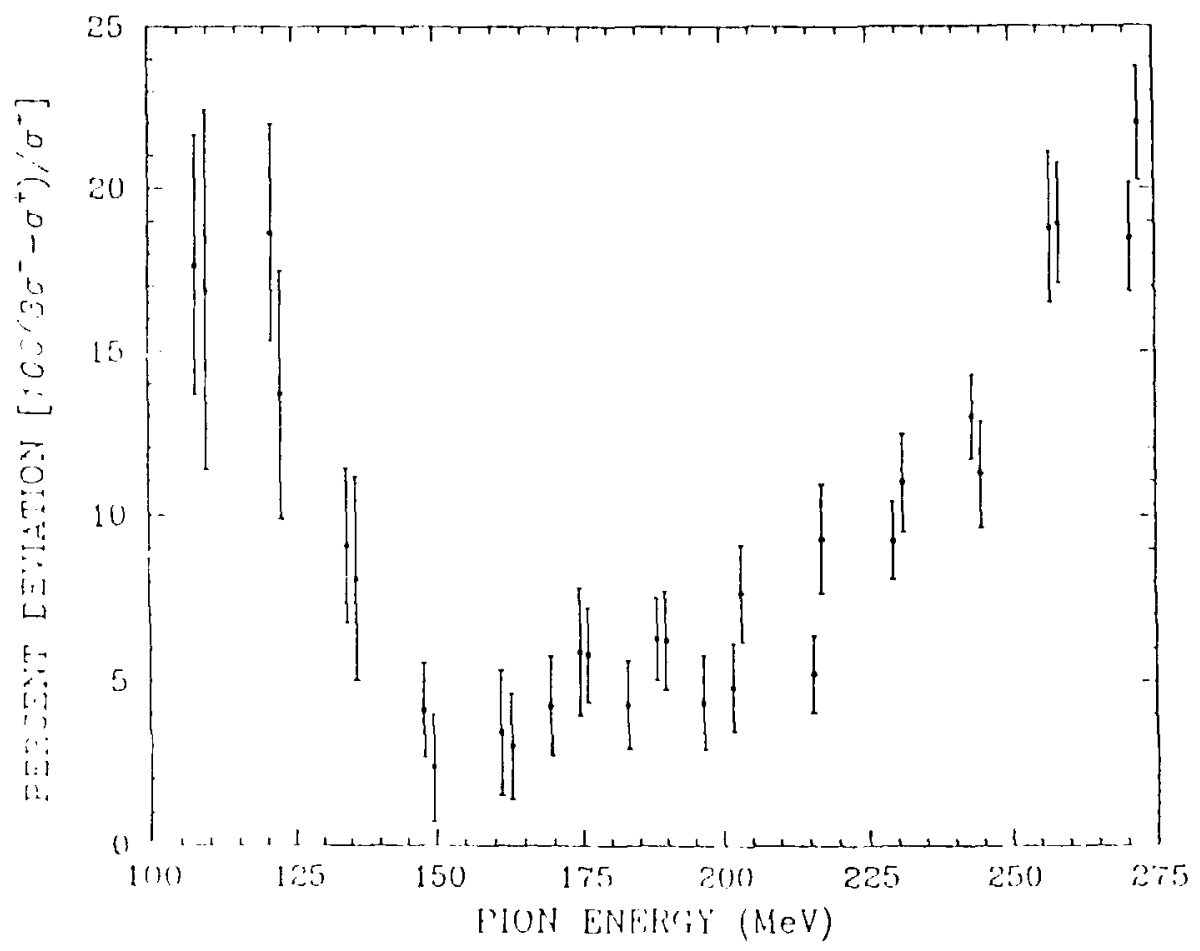

Figure 1.9: Percent deviation $\left[3 \sigma\left(\pi^{-} p\right)-\sigma\left(\pi^{+} p\right) / \sigma\left(\pi^{+} p\right)\right] \cdot 100$. 


\subsubsection{The Reactions $\mathrm{p}+\mathrm{d} \rightarrow \pi^{+}+{ }^{3} \mathrm{H}$ and $\mathrm{p}+\mathrm{d} \rightarrow \pi^{\circ}+{ }^{3} \mathrm{He}$}

Ruderman ${ }^{67}$ was probably the first to suggest the use of these reactions for testing charge independence. If charge independence is assumed, the ratio

$$
R=\sigma\left(p+d \rightarrow \pi^{+}+{ }^{3} H\right) / \sigma\left(p+d \rightarrow \pi^{0}+{ }^{3} H e\right)=2.0
$$

a value obtained easily from the isospin Clebsch-Gordon coefficients. Under the lyy pothesis that the transition matrix associated with these two reactions does not depend upon isospin quantum numbers, $R$ is given by:

$$
R=\left(\frac{\langle 1 / 2,1 / 2 \mid 1,1 / 2,1,-1 / 2\rangle}{\langle 1 / 2,1 / 2 \mid 1,1 / 2,0,1 / 2\rangle}\right)^{2}=\left(\frac{\sqrt{2} / \sqrt{3}}{-1 / \sqrt{3}}\right)^{2}=2
$$

where the Clebsch-Gordon coefficients have been give according to the notation $\left\langle j, m \mid j_{1}, j_{2}, m_{1}, m_{2}\right\rangle$.

Similarly, the analyzing power of the two reactions should be equal, i.e.,

$$
\Delta A=A_{y_{0}}\left(0,{ }^{3} \mathrm{H}\right)-A_{y_{0}}\left(\theta,{ }^{3} \mathrm{Hc}\right)=0
$$

at all angles. It is obvious, but it seems not to have been noticed before that this difference of analyzing powers should be a particularly sensitive measure of the type IV charge independence breaking force of Henley, since it is a spin dependent force.

Several measurements of $R$ have been made before. Their results range from 2.05 to 2.58 . No measurements of $\Delta \mathrm{A}$ have been reported in the literature beforc the ones which form the subject of the present dissertation. We postpone a detailed discussion of this reaction to a later section.

From the above review of the many experiments done to test isospin invariance we notice that most tests relate to the weaker invariance, namely charge symmetry. Few tests have ever been done to test charge independence. The one precision test which has been done, and which does reveal charge independence breaking unambiguously relates to a very special situation. It measures the difr. rence in ${ }^{1} S_{0}$ scattering lengths of the $n n$ (or pp) and the np systems. It sheds no 
light on the type IV charge independence breaking forces. There are essentially no precision experiments at intermediate energies which address the question of charge independence. This is the question we have chosen to address in this dissertation. 


\subsection{Criteria for choice of an experiment.}

In the attempt to design an experiment which would give meaningful information about charge indepentence, there are two main considerations which affect the choice. The first is theoretical in nature and has already been touched upon in a previous section. It is worthwhile, however, to consider it in some more detail, as it plays a crucial role in the correct interpretation of the experimental data. The idea behind each experiment is to find an unambiguous signature of the isospin nonconservation in the strong sector of the interaction between hadrons. Whatever observable is chosen, the expectation is that, even if an effect is present, it is bound to be small. On the one hand it is suggested by all the evidence that in nuclear physics isospin symmetry 'works'. On the other hand, the large rescaling of the quark masses, which takes place upon hadronization, suggests too that, accidental as it may be, isospin has a good chance of surviving as an almost good symmetry even in a reformulation of nuclear theory in terms of the dynamics of its elementary consituents. In this connection, a very simple argument gives some hints about the problems associated with the correct interpretation of the data. The strength associated with the electromagnetic interaction is of the order of its coupling constant, $\alpha$, i.e. $\approx 1 \%$. If we take as a scale of the isospin breaking at a 'elementary' haclronic level the fractional difference betwcen the constituent quark masses $\left(m_{u}-m_{d}\right) /\langle m\rangle$, we obtain a number of the same order of magnitude, i.e. $\approx 1 \%$. However, the two interactions have different dependence upon the energy and momentum variables of the particles involved. In an experimental observable the strong and the electromagnetic effects do not simply add incoherently, but also produce an interference term. In absence of a fundamental theory for the observable, which would enable one to calculate both the Coulomb and the Coulomb-strong interference contributions quantitatively, the safest approach to handle Coulomb contributions is to try to observe phenomena in which Coulomb effects are expected to be small. If this condition is satisfied, a wrong estimate of 
the interference term, or even its neglect, will not affect the interpretation of the data significantly.

From the pure experimental point of view, the requirements are those generally imposed whenever the evidence for a small effect is sought by means of a comparison between two different reactions. Small differences between the observables measured in the two cases should tell us something about symmetry breiting. Therefore a careful analysis of all the possible sources of bias introducen in the final results by the experimental method employed is needed. To Uh. purpose, the simplest approach to minimize systematic effects is to make as few changes as possible in the experimental conditions in the two measurements to be compared. 'The best choice would be one which involves a comparison between cwo reactions which have the same initial state and which have final states whose Hetection involves a minimal change in the ecpuipment used.

These considerations lead us to the experiment which is the subject of this disisertation. It involves the comparison between the two reactions:

$$
p+d \longrightarrow{ }^{3} \mathrm{II}+\pi^{+}
$$

and

$$
p+d \longrightarrow{ }^{3} \mathrm{He}+\pi^{0}
$$

The observables to be measured are the crisss section ratio:

$$
R(\theta)=\frac{d \sigma\left(\theta,{ }^{3}[1), d \Omega l\right.}{d \sigma\left(\theta,{ }^{3} \mathrm{He}\right) / d \Omega}
$$

and the analyzing power difference:

$$
\Delta A_{y 0}(\theta)=A_{y^{0}}\left(\theta,{ }^{3} \mathrm{II}\right)-A_{\left.y^{\prime}\right)}\left(\theta,{ }^{3} \mathrm{IIe}\right)
$$

Where the analyzing power is defined as:

$$
A_{\nu^{0}}(\theta)=\frac{\sigma_{1}}{\sigma_{i}+\theta^{\prime}} \cdot
$$


Our primary emphasis is on a precision measurement of $\Delta A_{y 0}$, because of its expected sensitivity to the type IV CI breaking interactions. The advantages of choosing the ratio $\mathrm{R}$ as the observable to be measured have already been discussed. This choice was made by all the previous experiments. $\triangle A_{y 0}$ was not considered before because polarized proton beams were not available to most of the previous experiments. In many respects $\Delta A_{v 0}$ provides a much more suitable observable for a high precision test. In fact, since $A_{y 0}$ is a ratio between cross sections, it is relatively insensitive to beam normalization and absolute efficiency of the detector system. Many additional uncertainties, such as variations in beam polarization, cancel out or introduce much smaller errors in the quantity $\Delta A_{y_{0}}$ if the measurements of the two reactions are alternated frequently.

\subsubsection{Choice of the incident energy.}

In order to facilitate an eventual theoretical explanation of the charge indepcndence breaking effects observed, it is advisable to choose the parameters of the experiment in such a manner as to minimize the effects of both Coulomb and hadronic distortions in both the incoming and outgoing channels. Coulomb distortions in both channels and hadronic distortions in the incoming channel require that the experiment be done at as high an energy as possible commensurate with the other objectives of the experiment. However, because of the resonant $\pi \mathrm{N}$ interaction in the outgoing channel, it is best to avoid outgoing pion energies near the (3.3) resonance, i.e., in the region $180 \pm 25 \mathrm{MeV}$. This could be done by choosing $T_{p} \leq 500 \mathrm{MeV}$ or $\geq 700 \mathrm{MeV}$. Clearly $T_{p} \geq 700 \mathrm{MeV}$ is to be preferred because it also minimizes Coulomb effects and incident channel hadronic distortions. Our experiment was done at $\mathrm{T}_{p}=730 \mathrm{MeV}$, which was the highest inergy at which a polarized proton beam was made available to us at LAMPF. At this energy the outgoing pion energy was $216 \pm 2 \mathrm{MeV}$. Several of the earlier experiments were done with pion energies much closer to $180 \mathrm{MeV}$. 


\subsubsection{Choice of the kinematical point.}

In order to do a precision experiment one likes to work at a kinematical point at, which derivatives with respect to the many experimental paraneters are at or near their minima. This requirement is generally impossible to satisfy and compromises have to be nlade. For aximple, one would like to study the reactions in question at angles where $\mathrm{d}^{\prime} \mathrm{T}_{3} / \mathrm{d} \theta$ is a minimum in onder to have the best energy resolution. This would dictate the choice of a forward angle. However forward angles are bad because of many reasons. Fom a theoretical point of view, they are bad because they maximize Coulomb effects. From the experimental point of view, they are undesirable because it is at forward angles that both the differential cross sections and the analyzing powers change rapidly both with incidcut energy and angle (see Figs. 1.10 and 1.11 ). Thus, for example, at $\theta_{\mathrm{cm}}(\pi) \approx 7^{\circ}, \mathrm{d} \sigma / \mathrm{d} \Omega$ changes almost twice as fast with entergy $(\approx 0.7 \%$ per $\mathrm{MeV})$ as it dons at $\theta_{\operatorname{cm}}(\pi)=$ $130^{\circ}(\approx 0.4 \%$ per $\mathrm{MeV})$. The angular dependence of the cross sections is of course much weaker at back angles. For example at $800 \mathrm{MeV}$ (sce Fig. 1.10), for angles $O(\pi)<45^{\circ}$ the cross sections fall at the rate of more than $20 \%$ per degree whereas at $\theta(\pi)=130^{\circ}$, the rate of change is $\langle 0.5 \%$ per dogree. Similar observations can be made about $A_{y o}(\theta)$ (see Fig. 1.11). It is obvious that back angles around $O(\pi) \approx 130^{\circ} \pm 10^{\circ}$ are to be much preferrod over forward angles from the point of view of minimizing effects due to the stability of the beam cnergy and direction.

Considerations of particle identification also impose strict conditions on the choice of angles. In Figs. 1.12-1.16, we display the detected particle nagnetic rigidity, momentum $/ z$, for several experiments. Wr notice that while the ${ }^{3}$ Ile rigiclities are such that they do not interfere with any other heavy particles (except, with pions and highly inclastic scatherngs), triton rigidilies are in the sane general region as the elastically scatlered protustand recoil denteroms, both of which have prolific yields. One must therefore avid the icinity of the crossing points at which these contaminant particles will food the mantiotir spetrometer and do havoc to 
even the best particle identification system. We note parenthetically, that one of the experiments reported in the literature ${ }^{80}$ was run almost precisely at such an undesirable angle at which elastic protons would overwhelm tritons in the focal plane of the spectrometer and another experiment ${ }^{78}$ was run at an angle at which pion yield from the $\mathrm{pp} \rightarrow \mathrm{d} x^{+}$reaction (from quasi free protons in deuterium) would compete in the focal plane with ${ }^{3} \mathrm{He}$ particles. In our choice of angle $\left(\theta_{L}\right.$ $12^{\circ}$ ) we have taken care to be equally far away from protons and deuterons from elastic scattering (see Fig. 1.16). Fortunately, this choice also keeps us far away from pions from both the pp $\rightarrow \mathrm{d} \pi^{+}$and $\mathrm{pd} \rightarrow \mathrm{t} \pi^{+}$reactions. 


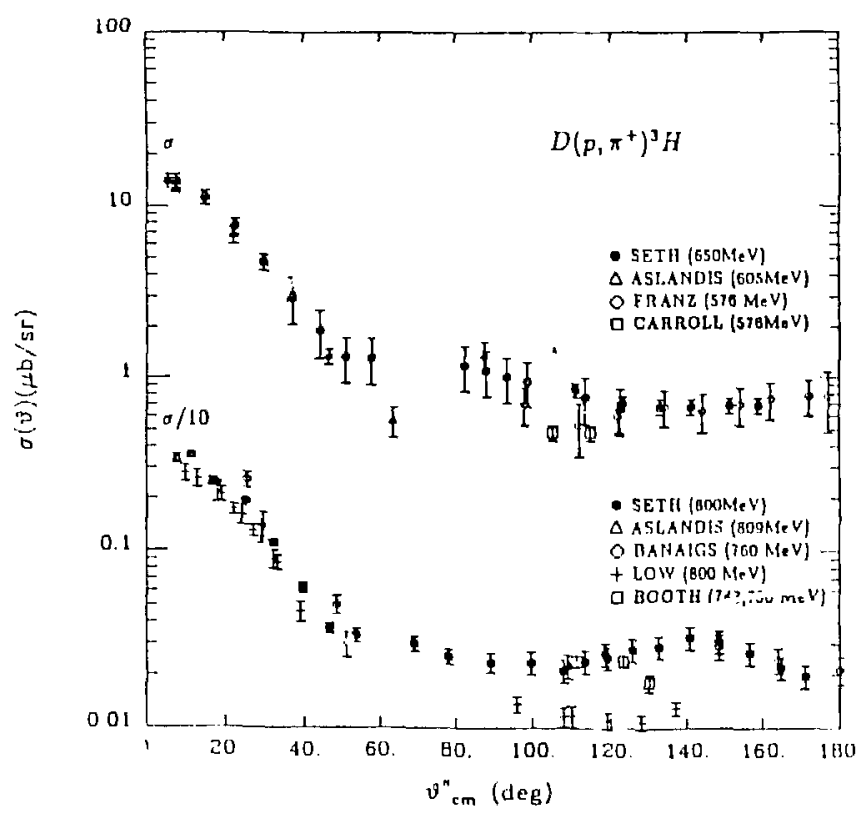

Figure 1.10: Differential Cross section for the reaction $p+d \rightarrow{ }^{3} \mathrm{II}+\pi^{+}$. The data are from Refs. 68-72 and 90.

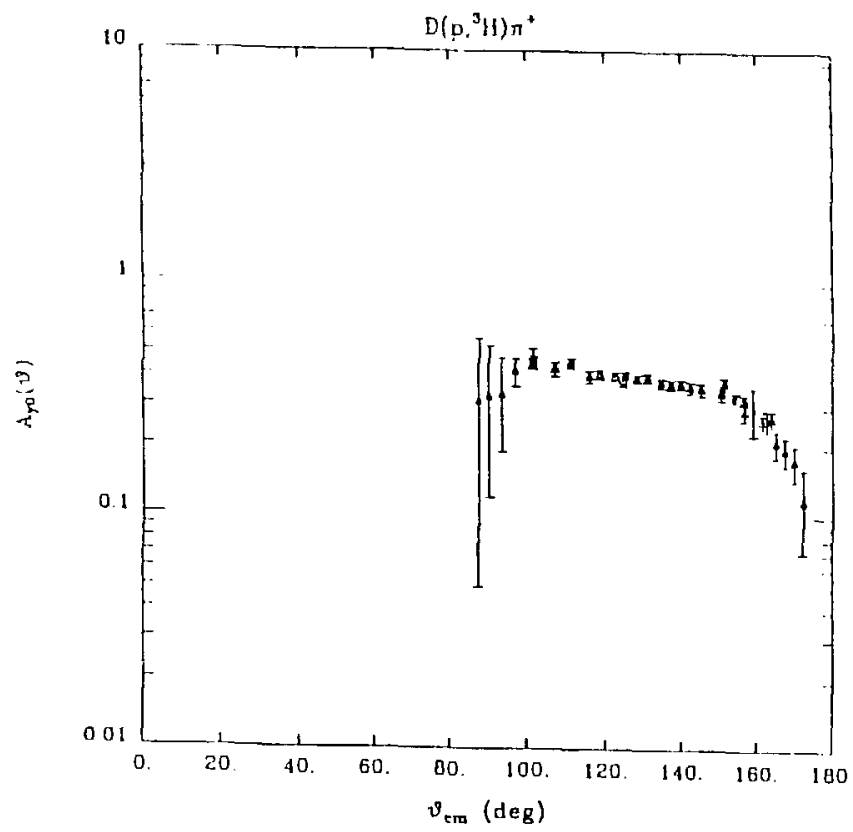

Figure 1.11: Analyzing power for the reaction $p+d \rightarrow{ }^{3} \mathrm{II}+\pi^{+}$at $\mathrm{T}_{\mathrm{p}}=700 \mathrm{MeV}$. (from Ref.90) 


\subsubsection{Choice of the target.}

Apart from the choice of incident proton energy and outgoing particle angle, there are several other criteria that should be kept in mind in such a measurement.

The first of these is the choice of a target. In order to minimize the yield from undesirable reactions it is necessary to keep material in the target, other than deuterium, at a minimum. It can be easily shown that this immediately rules out the use either of a solid target (for example $\mathrm{CD}_{2}$ ) or a gaseous target at room temperature and pressure (container wall too thick compared to $\mathrm{D}_{2}$ content in the target). Of the two possibilities super-cooled $\mathrm{D}_{2}$ gas at high pressure or liquid deuterium, liquid deuterium is preferable because it gives a much better ratio of deuterium to container material. $\mathrm{LD}_{2}$ is indeed the target of choice in most experiments, including ours.

Ideally the target should be as thin as possible in order to keep to a minimum the difference in energy losses suffered by ${ }^{3} \mathrm{Hi}$ and ${ }^{3} \mathrm{He}$ as they exit from the target. In practice this requirement is compromised by the mechanical design of the $\mathrm{LD}_{2}$ target as well as the necessity to keep $\mathrm{LD}_{2} /$ container mass ratio high, and the signal counting rates adequate for the statistical accuracy desired. Target thicknesses used in different experiments range froint $\approx 1 \mathrm{~cm}$ to $\approx 3 \mathrm{~cm}$. The thickness of our $\mathrm{LD}_{2}$ target was intended to be $\approx 1 \mathrm{~cm}$ but ended up being approximately $2.5 \mathrm{~cm}$. 


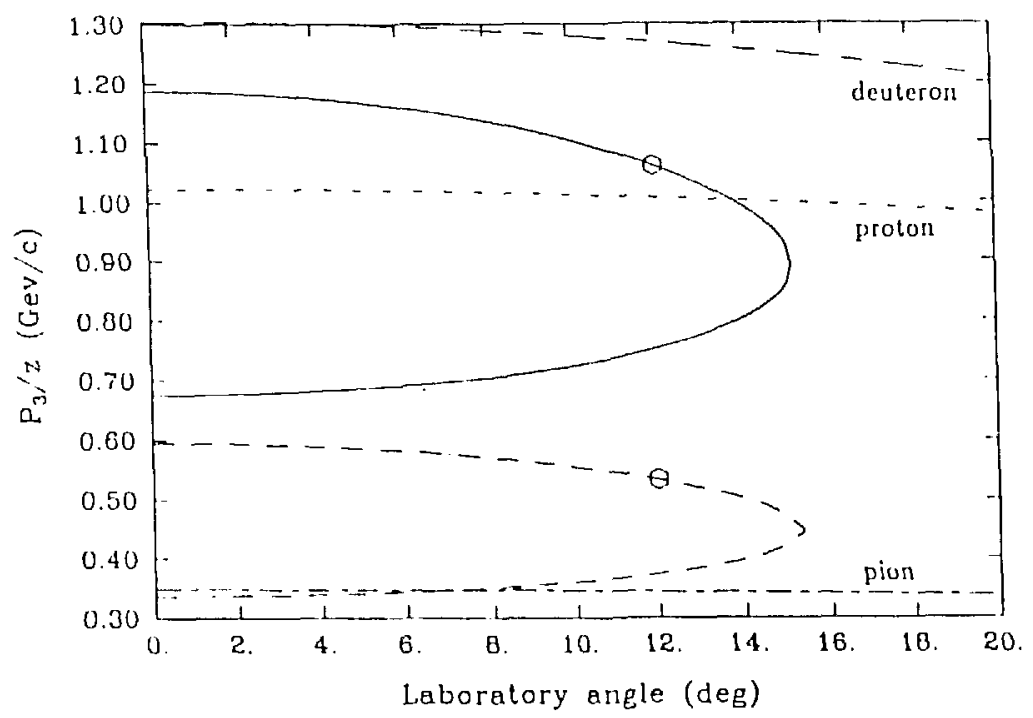

Figure 1.12: Kinematics for the reaction $\mathrm{p} \mathrm{d} \rightarrow{ }^{3} H\left({ }^{3} H e\right) \pi^{+}\left(\pi^{0}\right)$ as measured by Crewe et al.(Ref. 75,76 ).

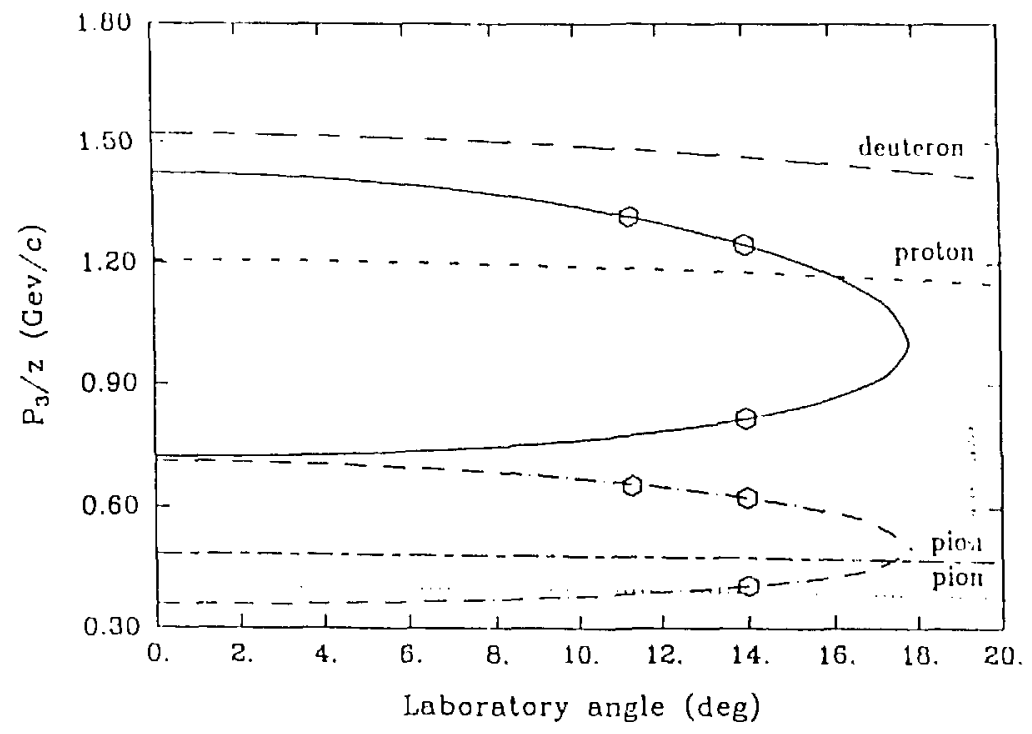

Figure 1.13: Kinematics for the reaclion p $\mathrm{d} \rightarrow{ }^{3} I I\left({ }^{3} H e\right) \pi^{+}\left(\pi^{0}\right)$ as measured by Ilarting et al.(Rcf, 77, 78). 


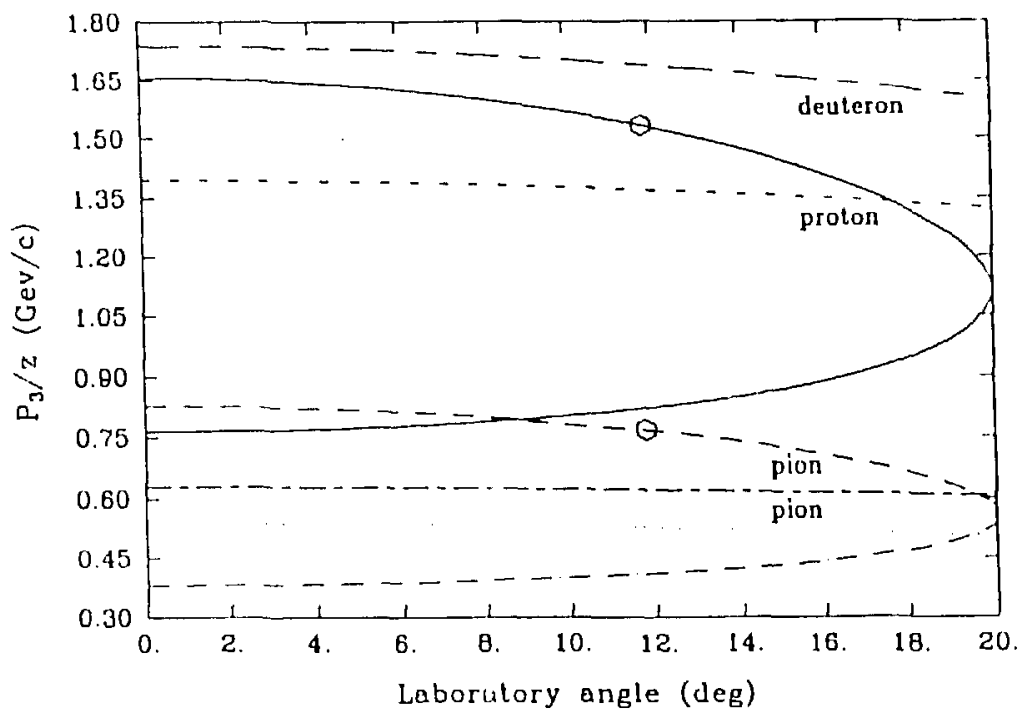

Figure 1.14: Kinernatics for the reaction $\mathrm{p} \mathrm{d} \rightarrow{ }^{3} H\left({ }^{3} H e\right) \pi^{+}\left(\pi^{0}\right)$ as measured by Booth et al.(Ref. 79)

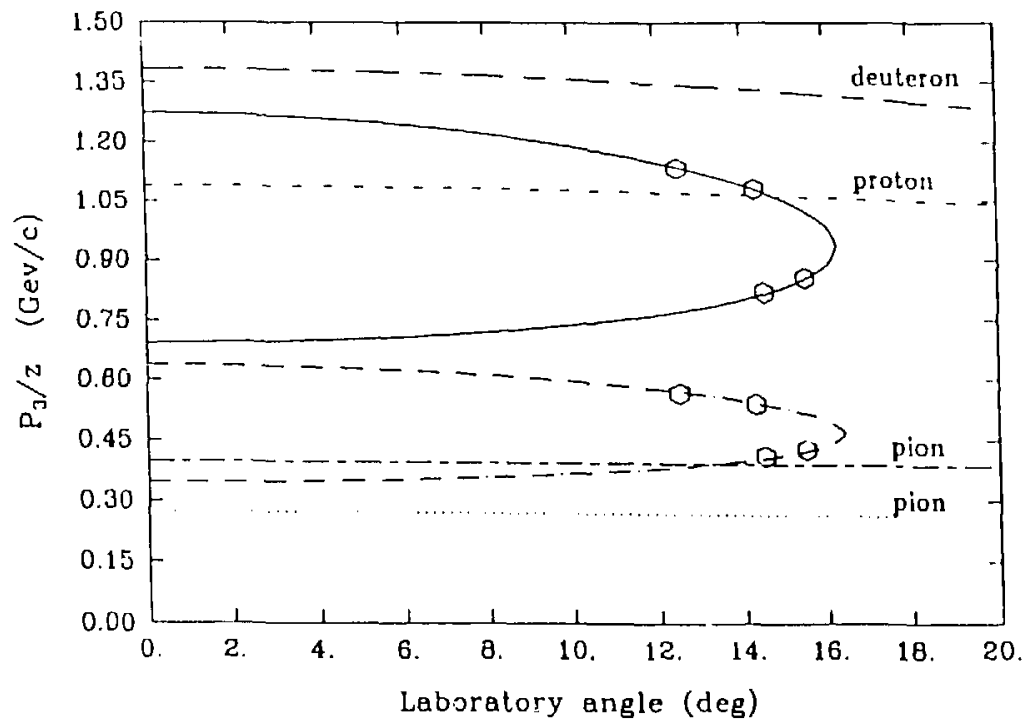

Figure 1.15: Kincmatics for the reaction $\mathrm{p} \mathrm{d} \rightarrow{ }^{3} H\left({ }^{3} H e\right) \pi^{+}\left(\pi^{0}\right)$ as measured by Silverman et al.(Ref. 80) 


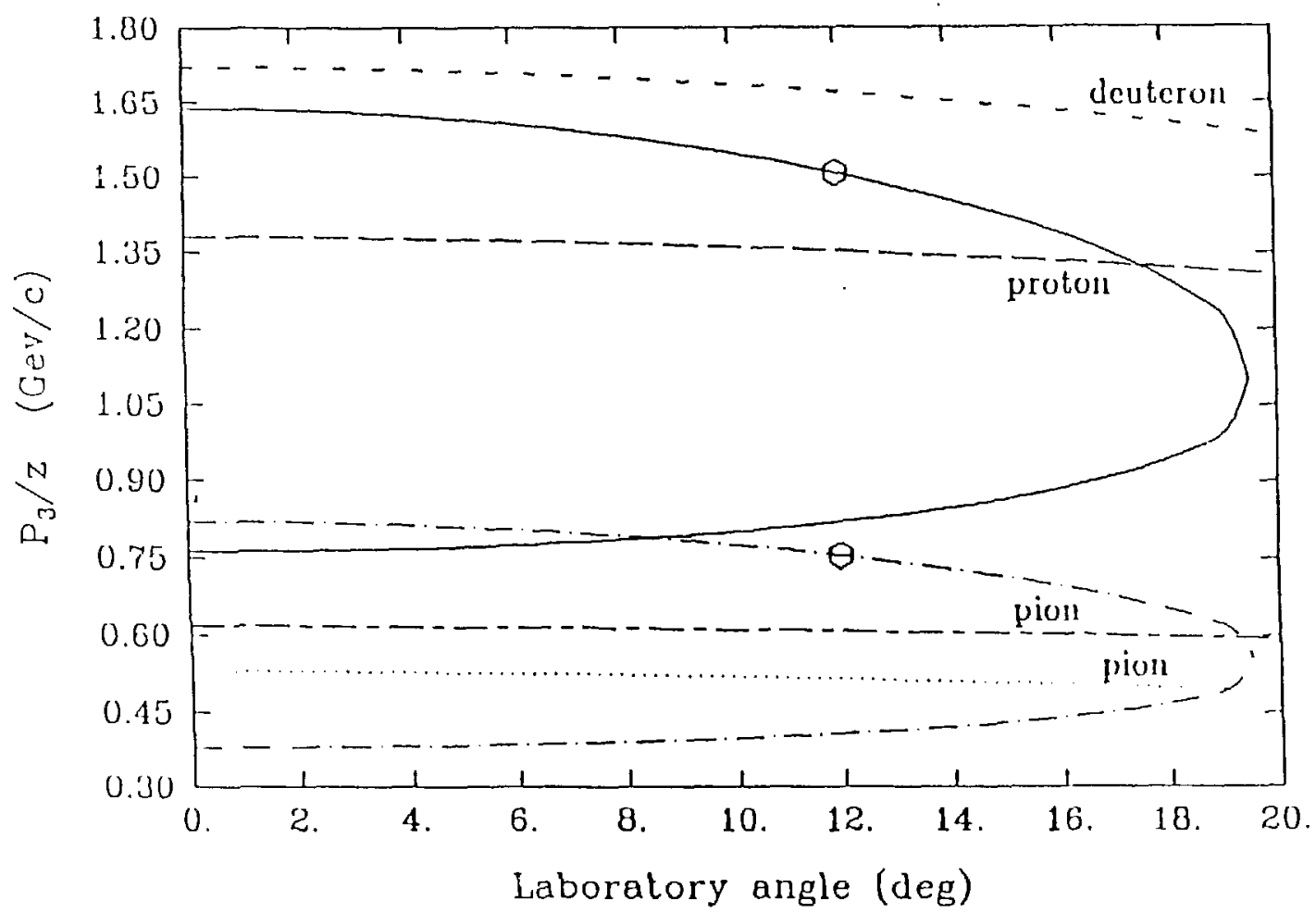

Figure 1.16: Kinematics for the reaction $\mathrm{p} \mathrm{d} \rightarrow{ }^{3} H\left({ }^{3} \mathrm{He}\right) \pi^{+}\left(\pi^{0}\right)$ for the present experiment. 
1.9 Survey of previous neasurements of the ratio $R$.

We now wish to discuss the several measurements of $R$ which have been reported in the literature (see Table 1.7). We shall examine these experiments critically in order to determine how we can improve our own experiment.

1. Bandtel, Frank and Moyer (Berkeley,1957)

This experiment, done with the $340 \mathrm{MeV}$ proton beam of the Berkeley 184 inch synchrotron and a $\mathrm{LD}_{2}$ target, was the forerunner of all such experiments. It is also the only experiment that did not have the advantage of a magnetic spectrometer. With no momentum sclections available $\mathrm{dE} / \mathrm{dx}-\mathrm{E}$ scintillation counters provided minimally successful particle identification. 'The result was that the authors could only conclude that $R$ was "between 2.0 and 2.6 ".

2. Crewe et al. (Chicago,1959-60) ${ }^{75,76}$

This was the first experiment ${ }^{77}$ to use a magnetic spectrometer. Using the $450 \mathrm{MeV}$ proton beam from the Chicago synchrocyclotron and $\mathrm{CD}_{2}$ and $\mathrm{C}$ targets these authors measured differential cross sections for the two reactions at $\theta_{L}\left({ }^{3} \mathrm{HI},{ }^{3} \mathrm{He}\right)$ between $8^{\circ}$ and $13^{\circ}$. A weighted mean of $R=1.91 \pm 0.25$ was obtained. The errors are presumably only statistical.

The experiment was refined and repeated ${ }^{76}$ at $\theta_{L}=12^{\circ}$ to obtain $R\left[\theta_{c m}(\pi)=140^{\circ}\right]=2.17 \pm 0.13$. The earlier results at other angles were reanalyzed to obtain $R\left(135^{\circ}\right)=2.08 \pm 0.22$ and $R\left(145^{\circ}\right)=2.34 \pm 0.37$. The quoted errors are essentially statistical.

From our experience it would appear that there are two main problems with this experiment. The first is the relative inadequacy of the $\mathrm{dE} / \mathrm{dx}-\mathrm{E}$ system alone to do particle identification at the required level. The second difficulty relates to the method used to determine the momentum acceptance of we detection system. No account of the change in acceptance between ${ }^{3} \mathrm{H}$ and ${ }^{3} \mathrm{He}$ detection appcars to have becí taken. Although no details are given, it would appear that the $\mathrm{CD}_{2}$ target gave a large yield from the carbon contaminant and the $\mathrm{C}$ contaminant 
Table 1.7: Experimental results for $R$.

\begin{tabular}{|c|c|c|c|c|c|c|c|c|}
\hline Authors & Target & $\begin{array}{c}T_{p} \\
\mathrm{HeV}\end{array}$ & $\begin{array}{l}T_{3} \\
\mathrm{Mev}\end{array}$ & $\begin{array}{l}T_{\pi^{+}} \\
\mathrm{MeV} \\
\end{array}$ & $\begin{array}{c}\theta_{L}^{3} \\
\operatorname{deg} \\
\end{array}$ & $\begin{array}{l}\theta_{c r n}^{\pi^{-}} \\
\operatorname{deg}\end{array}$ & $R$ & $\begin{array}{l}\text { Departure } \\
\text { from CI } \%\end{array}$ \\
\hline Crewe et al. ${ }^{(69)}$ & $\mathrm{CD}_{2}-\mathrm{C}(.8 \mathrm{~mm})^{\top}$ & 450 & 194 & 122 & 12.0 & 140.0 & $2.17 \pm .13$ & $8.5 \pm 6.5$ \\
\hline \multirow[t]{3}{*}{ Harting et al. ${ }^{(71)}$} & $\mathrm{LD}_{2}(0.8,2.5 \mathrm{~cm})$ & 591 & 292 & 164 & 11.3 & 128.0 & $2.26=.11$ & $13.0 \pm 5.5$ \\
\hline & $\mathrm{LD}_{2}(0.8 \mathrm{~cm})$ & 591 & 264 & 196 & 14.0 & $113.0^{\S}$ & $2.10 \pm .14$ & $5.0 \pm 7.0$ \\
\hline & $\mathrm{LD}_{2}(0.8 \mathrm{~cm})$ & 591 & 117 & 346 & 14.0 & $37.0^{*}$ & $2.05 \pm .09$ & $2.5 \pm 4.5$ \\
\hline Booth et al. ${ }^{(2)}$ & $\mathrm{D}_{2}$ (gas) & 743 & 388 & 221 & 11.7 & 130.0 & $2.00 \pm .30$ & $0.0 \pm 15.0$ \\
\hline \multirow[t]{4}{*}{ Silverman et al. ${ }^{(73)}$} & $\mathrm{LD}_{\mathbf{2}}(0.9 \mathrm{~cm})$ & 500 & 221 & 145 & 12.5 & 115.6 & $2.40 \pm .20$ & $20.0 \pm 10.0$ \\
\hline & & & 201 & 165 & 14.3 & $102.4^{\S}$ & $2.20 \pm .18$ & $10.0 \pm 9.0$ \\
\hline & & & 128 & 238 & 15.5 & $56.8^{\circ}$ & $2.58=.34$ & $29.0 \pm 17.0$ \\
\hline & & & 117 & 248 & 14.5 & $49.1^{\circ}$ & $2.52 \pm .26$ & $26.0 \pm 13.0$ \\
\hline This Experiment & $\mathrm{LD}_{2}(2.5 \mathrm{~cm})$ & 730 & 381 & 214 & 12.0 & 130.0 & $2.19 \pm .02$ & $9.6 \pm 1.1$ \\
\hline
\end{tabular}

† Large background correction due to carbon in $C_{2}$ target.

* Forward angle measurement. Rapid change in $\mathrm{d} \sigma / \mathrm{d} \Omega$ with angle. Results likely to be quite unreliable.

$\S$ Large elastic proton contamination expected. 
subtraction procedure is far from ideal.

\section{Harting et al. (CERN,1959-60) ${ }^{77,78}$}

This was the first experiment to use both a magnetic spectrometer and a liquid deuterium target. Using a $591 \pm 3 \mathrm{MeV}$ beam from the CERN synchrocyclotron these authors measured $R$. Time of flight was used as the means for particle identification. The authors note that while this provided good identification for ${ }^{3} \mathrm{He}$ particle, triton identification was plagued with large yield of recoil deuterons. In the first experiment at $\theta_{L}=11.3^{\circ}$, corresponding to $\theta_{c m}(\pi)=128^{\circ}$ these authors obtained $R=2.26 \pm 0.11$. The statistical errors were only about $2.5 \%$. Several systematic errors ranging from $1 \%$ to $4 \%$ were identified, but they were added to the statistical errors in quadrature to yield the final quoted errors of $\approx 5 \%$.

In a later set of experiments ${ }^{78}$, these authors measured $R$ at two other angles, $\theta_{L}=14^{\circ}\left[\theta_{c m}(\pi)=113^{\circ}\right]$ and $\theta_{L}=14^{\circ}\left[\theta_{c m}(\pi)=37^{\circ}\right.$. They report:

$$
\begin{aligned}
& R\left[\theta_{c m}(\pi)=113^{\circ}\right]=2.10 \pm 0.14 \\
& R\left[\theta_{c m}(\pi)=37^{\circ}\right]=2.05 \pm 0.09
\end{aligned}
$$

The value of $R\left[\theta_{c m}(\pi)=37^{\circ}\right]$ has been scaled upwards from the measured value $1.99 \pm 0.05$ in order to make an estimated correction for the $0.5^{\circ}$ difference in the c.m. angles for $\pi^{+}$and $\pi^{0}$.

The experiments of Harting et al. are perhaps the most carefully done experiments in the published literature so far. We note however that they introduce rather arbitrary weighting of data to account for target boiling. They suffer (particularly in the case of triton detection) from the fact that they used only time of flight for particle identification. The $\theta_{c m}(\pi)=37^{\circ}$ results are the least reliable because of the large variation of $\sigma(\theta)$ with $\theta$ at this angle and the use of an estimated correction factor to compensate for the c.m. angle change. In addition it would appear (see Fig. 1.13) that excessive number of pions from the prolific reaction $\mathrm{pp} \rightarrow \mathrm{d}+\pi^{+}$are accepted by the spectrometer at this angle. We also 
Iote that the authors do not provide any discussion of the differential acceptance of ${ }^{3} 11$ and ${ }^{9}$ He by the detection system.

4. Booth et al. (Berkeley,1963) ${ }^{79}$

In this experiment $R=2.00 \pm 0.10$ is reported for $\mathrm{T}_{p}=743 \mathrm{MeV}$, at $\theta_{L}=11.7^{\circ}$, $\theta_{\mathrm{c} m}(\pi)=130^{\circ}$. A gas target at $320 \mathrm{psi}$ and liquid nitrogen temperature $\left(77^{\circ} \mathrm{K}\right)$ was used. Particle identification was made by time of flight and $\mathrm{dE} / \mathrm{dx}$ measurements. Since the main purpose of the experiment was to study $\pi-\pi$ interaction, very few details of interest are presented in the paper and it is difficult to evaluate the precision of the experiment. An error of $10 \%$ is attributed to the cross sections by the authors on the basis of consistency check between consecutive runs. Even this should lead to an error of $\pm 14 \%$ in $R$ as we have listed in Table 1.7. The authors quote a final error of $5 \%$ without giving any explanation.

5. Silverman et al. (Saclay,1984)

In this experiment $R$ was measured at $\mathrm{T}_{p}=500 \mathrm{MeV}$ at $\theta_{\mathrm{cm}}(\pi)=49.1^{\circ}$, $56.8^{\circ}, 102.4^{\circ}$ and $11.5 .6^{\circ} . \mathrm{A} \mathrm{LD}_{2}$ target was used and particle identification was done by measuring both $\mathrm{dE} / \mathrm{dx}$ and time of flight of the outgoing particles which were momentum analyzed in a magnetic spectrometer. As listed in Table 1.7, these authors report values of $R$ ranging from $2.20 \pm 0.18$ to $2.58 \pm 0.34$ at different angles.

These experiment suffer from very poor choice of kinematical conditions. We have already pointed out that because of the rapid variation of cross sections at forward pion angles, these angles are unsuitable for precision measurements. We believe that for this reason the results at $\theta_{\mathrm{cm}}(\pi)=49.1^{\circ}$ and $56.8^{\circ}$ are not reliable. The other two angles, $\theta_{L}\left({ }^{3} \mathrm{H}\right)=12.5^{\circ}$ and $14.3^{\circ}$ do not represent wise choices of angles either. As Fig. 1.15 illustrates, elastically scattered protons should literally flood the spectrometer at $\theta_{L}=14.3^{\circ}$, and constitute a serious background even at $\theta_{L}=12.5^{\circ}$. Not only should this lead to bad conditions for particle identification but it should also have deleterious effect on chamber efficiencies, accidentals 
etc. We also note that in this experiment the momentum acceptance of the spectrometer was considerably smaller than the momentum spread of the ${ }^{3} \mathrm{He}$ 'peak', and three separate measurements had to be added to completely cover the 'peak'. Because of all the reasons mentioned, we believe that these experiments do not provide reliable measurements of $R$.

\section{Low et al. (LAMPF, 1981) ${ }^{69}$}

Low et al. have measured differential cross sections for $\mathrm{pd} \rightarrow^{3} \mathrm{He} \pi^{0}$ at 800 $\mathrm{MeV}$ in the angular range $\theta_{c m}(\pi)=10^{\circ}-130^{\circ}$. They used al $\mathrm{LD}_{2}$ target, a $\mathrm{C}$ magnet as crude spectrometer and did particle identification by time of flight. They also made measurement of $\mathrm{pd} \rightarrow{ }^{3} \mathrm{H} \pi^{+}$cross sections at three angles $\theta_{\mathrm{cm}}(\pi)=10^{\circ}$, $17^{\circ}$ and $30^{\circ}$. They obtain $R=2.09(12), 2.10(12)$ and $\geq 2.12(22)$ from these measurements. They also compare extrapolations of their ${ }^{3} \mathrm{He} \pi^{0}$ data to three datum points due to Aslanides(Saclay) ${ }^{68}$ for the ${ }^{9} \mathrm{H} \pi^{+}$reaction at $809 \mathrm{MeV}$ to obtain $R=2.06(8)$ at $\theta_{c m}(\pi)=20^{\circ}, 2.09(8)$ at $\theta_{\mathrm{cm}}(\pi)=25^{\circ}$, and $2.06(8)$ at $\theta_{c m}(\pi)=30^{\circ}$.

In our opinion these results suffer from the disadvantages common to forward angle measurements. Also comparison of experiments from two different laboratories can be hardly expected to give precision values of $R$. We believe that the error quoted for the grand average value of $R=2.08(2)$ can not be taken seriously.

\section{Dutty et al. $(\text { SIN }, 1981)^{82}$}

These authors have measured the differential cross sections for the reactions $\mathrm{n} \mathrm{d} \rightarrow{ }^{3} \mathrm{H} \pi^{0}$ and $\mathrm{nd} \rightarrow{ }^{3} \mathrm{He} \pi^{-}$for $\mathrm{T}_{n}$ between $400 \mathrm{MeV}$ and $590 \mathrm{MeV}$ by detecting ${ }^{3} \mathrm{H}$ and ${ }^{3} \mathrm{He}$ in a magnetic spectrometer. They find that the ratio

$$
R^{\prime}=\sigma\left(\mathrm{nd} \rightarrow{ }^{3} \mathrm{He} \pi^{-}\right) / \sigma\left(\mathrm{nd} \rightarrow{ }^{3} \mathrm{H} \pi^{0}\right)
$$

has an average value of $1.71 \pm 0.10$, instead of the 2.0 expected on the basis of charge independence. It is difficult to relate $R^{\prime}$ to the ratio $R$ measured in proton experiments without making a model for departures from charge symmetry in 
the two cases. To provide an illustration, if the entire difference from the isospin prediction of 2.0 is attributed to differences in wave functions of ${ }^{3} \mathrm{H}$ and ${ }^{3} \mathrm{He}$, then the value $R^{\prime}=1.7$ for the nd reaction would correspond to $R \approx 2.3$ for the pd reaction.

\subsection{The present experiment.}

The preceeding critical evaluation of earlier experiments enables us to demand the following conditions for our experiment:

a) The proton beam energy should be $\geq 700 \mathrm{MeV}$, the higher the better. The beam energy should be stable to well within $\pm 1 \%$.

b) The beam should have a high degree of transverse polarization, $P \geq 0.8$. It should be stable and continuously monitored with a precision of the order $\pm 1 \%$.

c) The target should be $\mathrm{LD}_{2}$ in a thin-wall flask. Preferably the target should be of uniform thickness of $\approx 1 \mathrm{~cm}$. The target should be stable, with no boiling or erratic changes in density.

d) The beam direction and intensity should be stable and continuously monitored.

e) It is highly destrable to have a stable monitor of beam-target interactions.

f) Particle identification should be done by both $\mathrm{dE} / \mathrm{dx}$ and time of flight measurements.

g) As far as possible particle detection conditions for ${ }^{3} \mathrm{H}$ and ${ }^{3} \mathrm{He}$ should be identical. Thus, for example, appropriate changes in chamber voltages and scintillation counter voltages should be made between ${ }^{3} \mathrm{H}$ and ${ }^{3} \mathrm{He}$ detection to give comparable pulse heights in the deteciors employed. 
h) Measurements of the two reactions should be alternated as many times as possible to cancel out the effects of long term changes in experimental conditions.

i) A Monte Carlo simulation of the experiment should be done in order to determine parameters which can not be directly measured, e.g., the effect of multiple Coulomb scattering and differential effects on detection efficiency due to different energy spreads in outgoing ${ }^{3} \mathrm{H}$ and ${ }^{3} \mathrm{He}$.

As will be seen later most of these conditions were realized in our experiment. A notable exception was item (e). No independent monitor of beam-target interactions was available.

\subsubsection{Difficulties in interpretation.}

At the outset we wish to note the simple fact that since no successful theories of pion production are presently available, we will not be able to submit our experimental results to immediate theoretical interpretation. In abserce of such a theory, we will not be able to apply reliable corrections for external electromagnetic effects. Knowing this, all we can do, and indeed do, is to choose the parameters of the experiment such that these effects are as small as possible.

In the present dissertation we will be able to present only a phenomenological interpretation of our results. A definitive interpretation will have to wait for the development of a reliable model of pion production in $\mathrm{p}-\mathrm{d}$ collisions.

\section{i}




\section{Chapter 2}

\section{The experimental method.}

The present experiment was performed at the high resolution spectrometer (HRS) at the Los Alamos Meson Physics facility (LAMPF). A schematic of the LAMPF accelerator complex is given in Fig. 2.1.

Details of the accelerator are given in Appendix A. In the following each component of the present experimental set-up will be discussed and a critical study of the experimental parameters which have the most significant effect on the observables measured will be performed.

\subsection{The beam.}

The polarized $\mathrm{P}^{-}$beam, which is accelerated simultaneously with the main $\mathrm{H}^{+}$beam in the LAMPF linear accelerator, is deflected in line $\mathrm{X}$ and tailored for use at the HRS in line $\mathrm{C}$. The schematic showing line $\mathrm{C}$ is displayed in Fig. 2.2.

During our experiment the beam polarization was of $\mathrm{N}$-type and the nominal kinetic energy of the beam was $733 \mathrm{MeV}$. The average beam current was in the range of 1-3 na. Details on the polarized proton source are given in Appendix B.

Beam intensity was monitored by means of two ion chambers $(E R 02$ and $E R 04)$ located inside the scattering chamber, immediately downstream of the target.

The beam polarization was measured by two independent techniques. The first, known as 'quench ratio' 82 method, employs the information of the ratio 
Figure 2.1: Schematic view of the LAMPF accelerator.

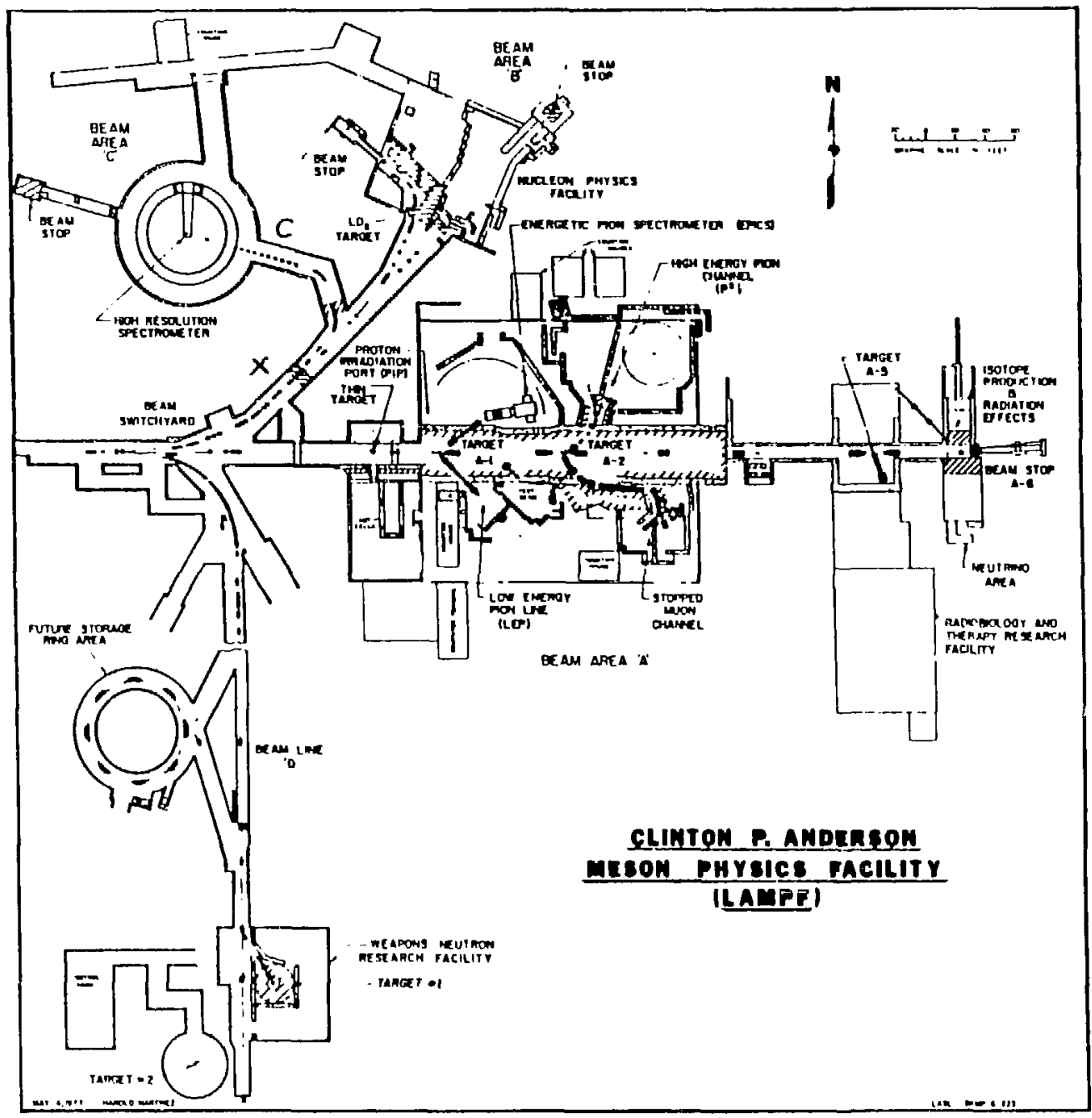


Figure 2.2: Schematic view of line C.

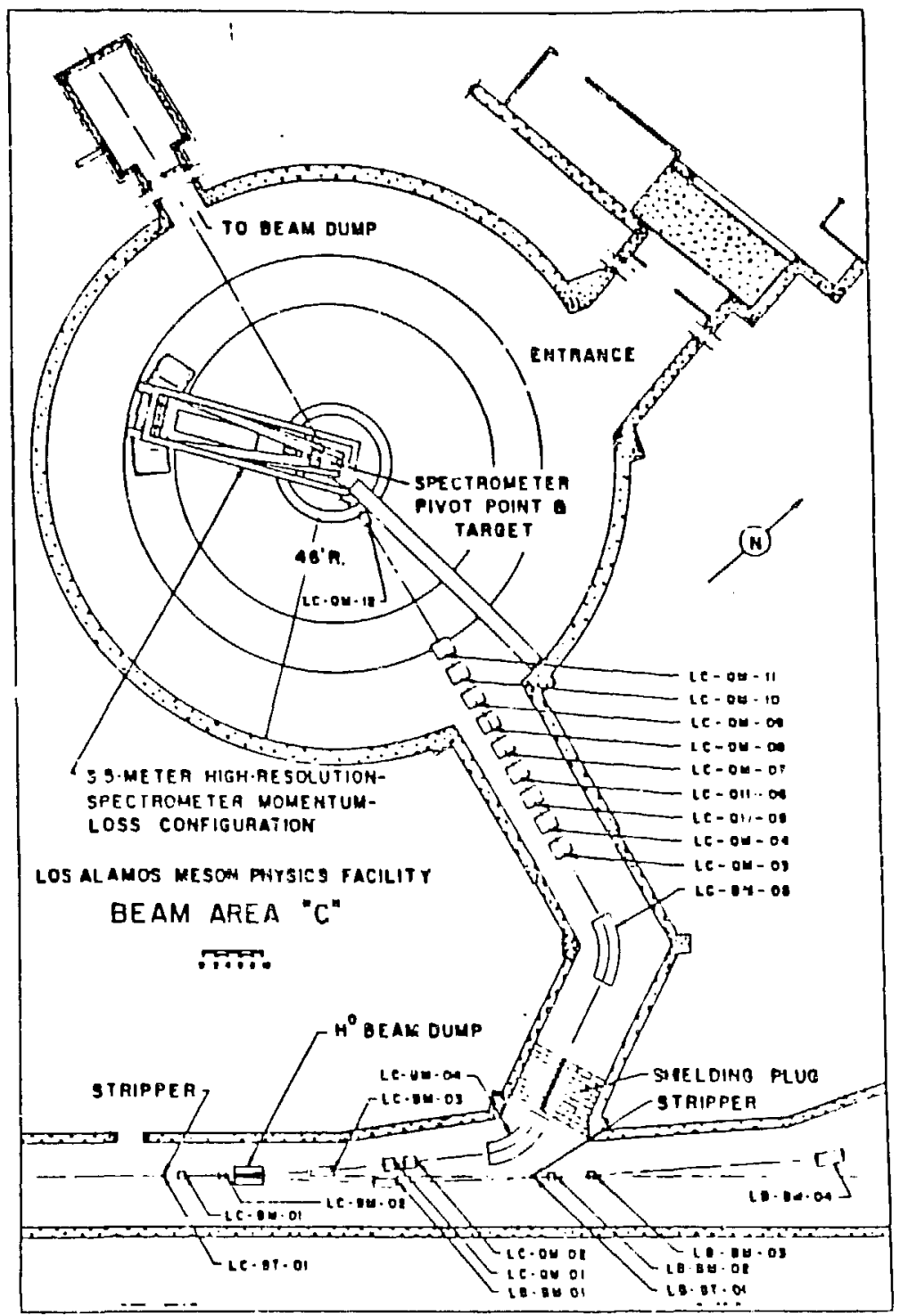


between the unpolarized beam current and the polarized one. At the end of each cycle of 'normal-reverse' polarization, the source is detuned so that only the unpolarized, background part of the beam is transmitted. The ratio of the beam intensities during this 'quenched' period and the 'unquenched' period gives the beam polarization:

$$
P_{B}=1-\frac{I_{Q}}{I_{P o l}}
$$

where:

$$
\begin{aligned}
I_{Q} & =\left(\frac{N(E R 04) \text { or } N(E R 02)}{N(\text { BeamGates })}\right)_{Q} \\
I_{P o l} & =\left(\frac{N(E R 04) \text { or } N(E R 02)}{N(\text { BeamGates })}\right)_{P o l}
\end{aligned}
$$

and

- $N(E R 04)$ or $N(E R 02)$ represent the readings of the scalers associated with the corresonding beam integrators,

- $N$ (Beam Gates) indicates the readings of the scalers which count the number of beam macropulses,

- the subscripts $Q$ and $P o l$ refer to the polarization status of the beam ( $Q$ corresponds to the 'quenched' beam and Pol corresponds to normal or reverse polarized beam).

Another method which is available to measure the beam polarization is the line $\mathrm{C}$ polarimeter ${ }^{83}$. In this polarimeter measurements are made of the left-right and up-down asymmetry in the yields of proton-proten elastic scattering. The leftright asymmetry provides a measursment of $\mathrm{N}$-type polarization (perpendicular to the scattering plane) and the up-down asymmetry provides a measurement of the S-type polarization (perpendicular to the beam direction in the scattering plane).

Each set of monitors is composed of a pair of scintillator counters positioned to detect the coincidence between two protons coming from elastic scattering at 
the angles $\left(\theta=19.1^{\circ}, \theta^{\prime}=63.7^{\circ}\right)$ corresponding to the maximum value of $A_{p}(\theta)$ in this energy region. The yields for left(up) and right(down) pairs are integrated and two quantities of interest $\langle P\rangle$ and $G_{h o r}$, are constructed:

$$
\langle P\rangle=\frac{1}{A_{p}} \cdot \frac{(1-\chi)}{(1+\chi)}
$$

where:

$\langle P\rangle$ is the average of the beam polarization for normal and reverse orienta. tion,

$A_{p}=0.507 \pm 0.008$ is the analyzing power for pp elastic scattering, $\chi=\sqrt{\frac{R_{\uparrow}}{L_{\uparrow}} \frac{L_{\downarrow}}{R_{\ddagger}}}$ where $L, \mathrm{R}$ refer to the corresponding pair of scintillators,

and

$$
G_{\text {hor }}=\sqrt{\frac{L_{\uparrow} L_{\downarrow}}{R_{\uparrow} R_{\uparrow}}}
$$

$G_{h o r}$ corresponds to the difference of effective solic angle between the left and right detector pairs. This parameter is interesting because its relative fluctuations give an indication of the corresponding fluctuations of the beam centroid in the horizontal plane.

The relationships given above also apply to the measurements of the S-type polarization by the change $L(R) \rightarrow U(D)$. The parameter $G_{v e r t}$ associated with the up and down detectors gives a measurement of the fluctuations of the beam centroid in the vertical plane intercepting the beam direction.

\subsection{The Target.}

Two main considerations constrained the choice of the deuterium target employed and its geometrical characteristics as used in the present experiment.

Pion production cross sections for the $p+d \rightarrow{ }^{3} H\left({ }^{3} H e\right)+\pi^{+}\left(\pi^{0}\right)$ are known to be quite small $\left(\approx 1 \mu \mathrm{b} / \mathrm{sr}\right.$ at $\left.\theta(\pi)=130^{\circ}\right)$. This rules out the use of $\mathrm{CD}_{2}$ solid target 
as well a cooled gas target for which the background from the $\left(p,{ }^{3} \mathrm{He}\left({ }^{3} \mathrm{H}\right)\right)$ reactions on carbon nuclei and nuclei of the container materiai would be the dominant trigger source. This made the choice of a liquid deuteriun. target mandatory.

Energy loss effects, which will be described in detail in a subsequent section, give the two criteria for the $\mathrm{LD}_{2}$ target design. The target should be as thin as possible, consistent with other requirements, e.g., counting rate, desired in the experiment, and the target should present a uniform thickness to the dispersed beam. The target used in the present experiment was designed by Mr. J. K. Novalk of MP-7. It consisted of a right circular cylinder of brass with mylar windows for beam entry and exit (see Fig. 2.3). The design volume for $\mathrm{LD}_{2}$ was $2.560^{\prime \prime}$ (6.50 $\mathrm{cm}$ ) diameter and $0.450^{n}(1.143 \mathrm{~cm})$ thickness. The target was designed to operate with a refrigerator (CTI model 1020) with a nominal cooling capacity of 10 watts at $20^{\circ} \mathrm{K}$. The normal operating pressure of $\mathrm{LD}_{2}$ was 15.0 psia.

The design target thickness was $1.143 \mathrm{~cm}$. However, because the target flask containing liquid deuterium at 15 psia was intended to operate inside a vacuum scattering chamber, its mylar wirldows were expected to deform as a consequence of the stress induced by the difference in pressure between their two sides. Before installation in the scattering chamber, the target was tested at 38 psia and a maximum deflection of the mylar windows was measured to be $1.080 \mathrm{~cm}$. From this measurement, the maximum deflection at the window center (normalized with respect to the window diameter and based on the assumption that the deformed window is segment of a spherical surface) for the operating pressure of 15 psia was calculated to be $0.541 \mathrm{~cm}$. It has been suggested ${ }^{84}$ tha 6 the actual value of the maximum deformation was likely to be somewhat more then that calculated in this manner, because of some permanent deformation produced by the initial test periormed.

In Table 2.1 the t'ickness distributions along a transversal direction are given for the two cases under consideration. The calculation was performed up to a deviation of $1 \mathrm{~cm}$ with respect to the target center,corresponding to the acceptance 
Table 2.1: Thickness distribution as a function of the coordinate $\rho$

\begin{tabular}{|c|c|c|}
\hline (mm) & $\begin{array}{c}\text { Thickness }(\mathrm{cm}) \\
(\mathrm{P}=15 \mathrm{psi})^{*}\end{array}$ & $\begin{array}{c}\text { Thickness }(\mathrm{cm}) \\
(\mathrm{P}=34 \mathrm{psi})\end{array}$ \\
\hline 0 & 2.210 & 3.200 \\
2 & 2.205 & 3.187 \\
4 & 2.192 & 3.165 \\
6 & 2.141 & 3.073 \\
8 & 2.103 & 3.004 \\
10 & 2.080 & 2.900 \\
\hline
\end{tabular}

* Pressure during the experiment.

of the HRS spectrometer for the horizontal coordinate at target. This distribution of target thicknesses seen by the beam generates an enhanced sensitivity to fluctuations in the position of the beam centroid with respect to the target center.

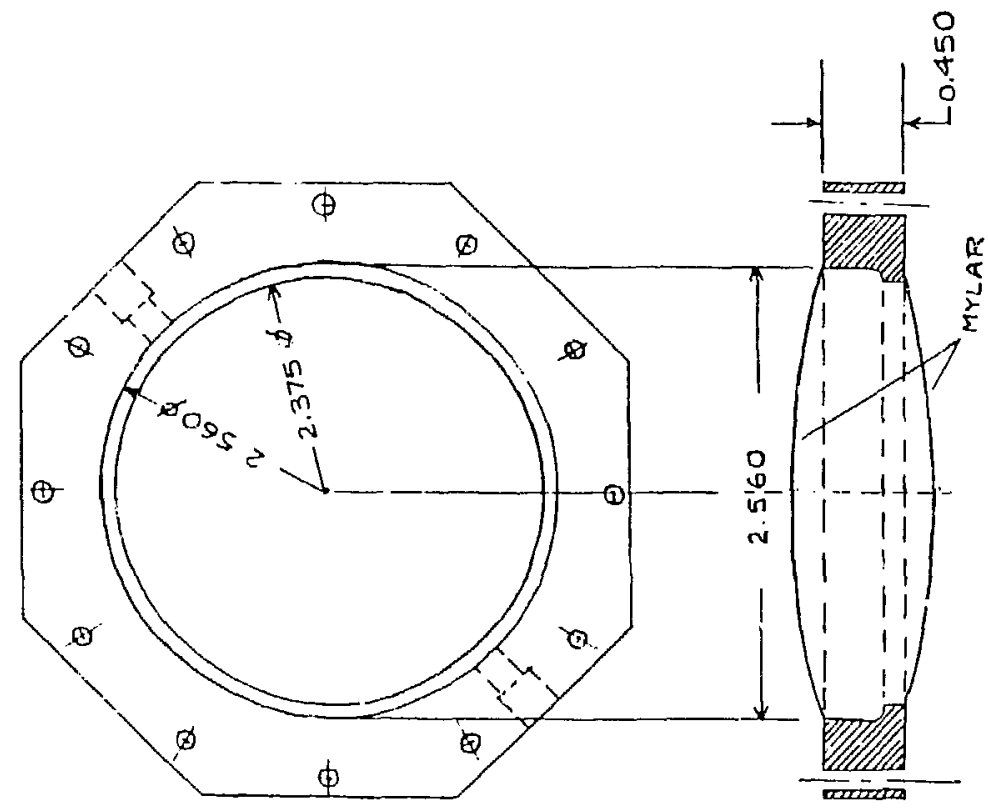

Figure 2.3: Schematic of $\mathrm{LD}_{2}$ target under 15 psi pressure. 


\subsection{The HRS spectrometer.}

The data acquisition was performed at the HRS Facility, employing the standard experimental set-up provided to detect a charged particle in the final state. This detection system performs momentum analysis and particle identification. Momentum analysis is done by a magnetic spectrometer ( which also acts as a 'momentum filter') and a set of multiwire drift chambers, which allow complete reconstruction of the trajectory of the detected particle.

The magnetic spectrometer ${ }^{85}$ is of a QDD (quadrupole-dipole-dipole) configuration. The high resolution which is provided by this system for most of the conventional inelastic scattering experiments ( $\leq 100 \mathrm{KeV}$ FWHM for proton scattering from heavy targets) is achieved through operation in the 'energy loss mode'. Details about this design aspect and on other characteristics of the sectrometer are given in Appendix C.

Fig. 2.4 gives a schematic representation of the detector system associated with the HRS magnetic spectrometer. It consists of 2 sets of multiwire drift chambers and five removable scintillator detectors. Table 2.2 summarizes the relevant geometrical parameters which characterize these detectors.

Table 2.2: Geometrical characteristics of the detectors associated with the HRS magnetic spectrometer.

\begin{tabular}{|c|l|}
\hline Detector & Size $(\mathrm{cm})$ \\
\hline$C_{i}^{*}$ & $60.0 \times 10.0$ \\
$S_{1}$ & $53.0 \times 9.8 \times 0.953$ \\
$S_{2}$ & $58.2 \times 10.7 \times 0.953$ \\
$S_{9}$ & $65.0 \times 12.0 \times 0.953$ \\
$S_{4}$ & $76.0 \times 13.0 \times 0.953$ \\
$S_{5}$ & $85.3 \times 14.4 \times 0.953$ \\
\hline
\end{tabular}

* All the drift chambers have this active area.

The front and rear drift chamber modules each consist of a pairs of planes 


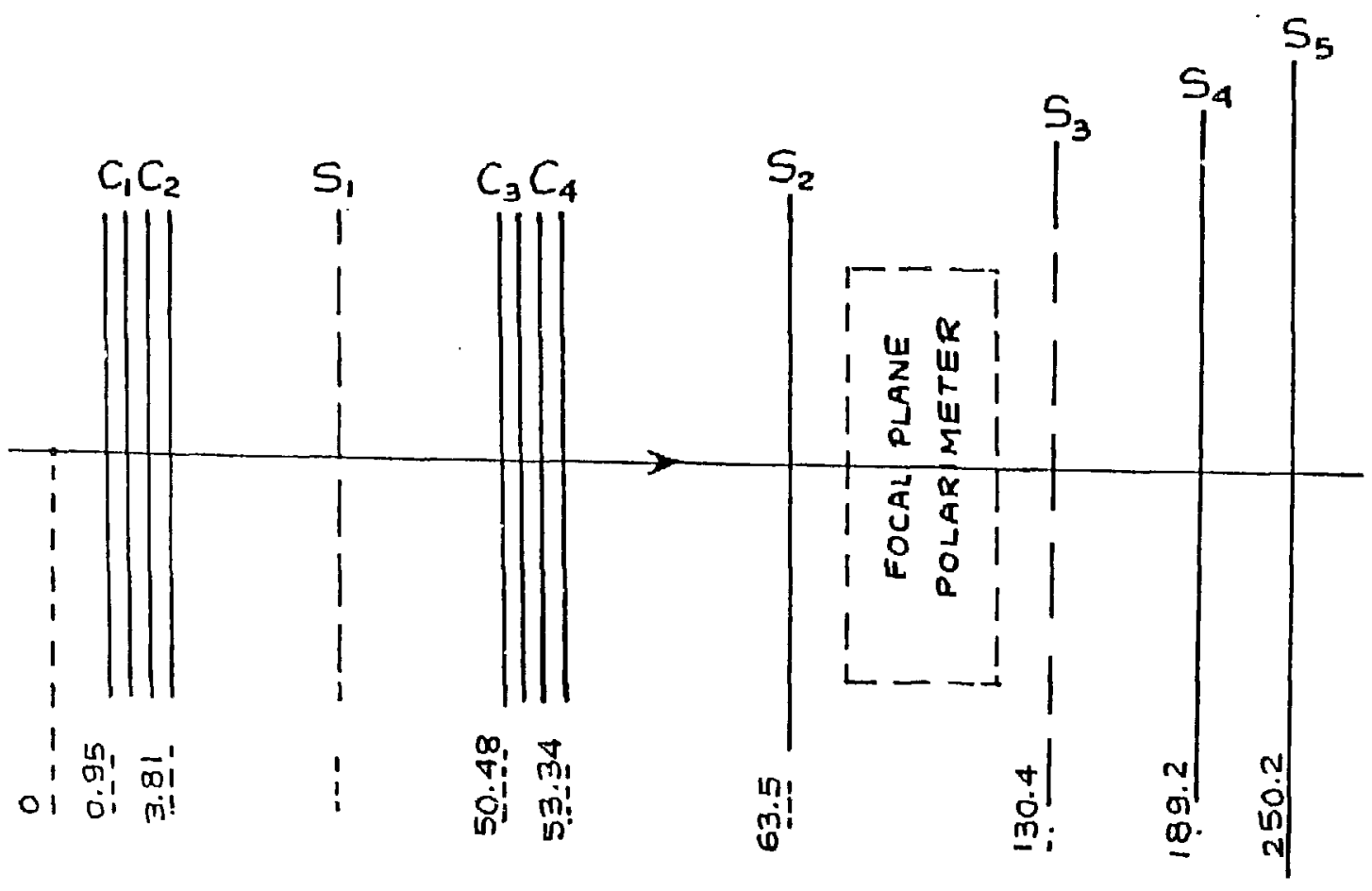

Figure 2.4: Schematic representation of the HRS detector system. Scintillators $\mathrm{S}_{1}$ and $S_{3}$ were not used in the present experiment and were moved out of position. 
(C1,C2 and C3,C4, see Fig. 2.4) sensitive respectively to the vertical and the horizontal position of the detected particle. In each set of adjacent chambers with parallel wires the corresponding anodes are positioned with a relative displacement of $.4 \mathrm{~cm}$ (length equivalent to one half of a drift cell). This configuration allows one to determine the sign with which the drift distance is combined with the wire position in the reconstruction of the trajectory associated with a given event. The sense wires are soldered on a printed circuit delay line. The two ends of each delay line, which are conventionally referred to as $\mathrm{N}$ and $\mathrm{P}$ respectively, are connected with constant fraction discriminators located near the detector system. More details on the technical charcteristics of these chambers can be found in refs.(86-87).

The scintillator detectors located beyond the drift chambers provide the hardware trigger which defines the occurrence of an 'event' in the detection system. This trigger condition is determined by the coincidence between the scintillators which are on line. During our experiment, the scintillators employed were $S_{2}, S_{4}$, $\mathrm{S}_{5}$. These counters also provided the TOF (time-of-flight) and the pulse heights for the particle identification system. The PID test adopted in our experiment involved the pulse height information associated with detector $S_{2}$ and the time of flight between $S_{2}$ and $S_{6}$. The light signals generated in each scintillator are collected at the two vertical ends, generally referred to as 'up' and 'down' respectively, by two photomultipliers. Their signals are sent to the electronic signal processor which is described in the next section.

\subsection{Signal processor for the HRS detector system.}

Signals coming from the phototubes at the two ends of the scintillators and from the delay lines associated with the drift chambers are sent to the electronics processor given in Fig. 2.5. This system has the twofold purpose of generating the coincidence $S_{2} \cdot S_{4} \cdot S_{5}$ and processing the signals coming from the detectors to obtain the data words used by the HRS event analyzer for the software reconstruction of 
the event.

A meantimer circuit generates a logical signal which defines an 'average time' between the times $\mathrm{T}_{\text {up }}$ and $\mathrm{T}_{\text {down }}$ defined by the leading edge of the pulses coming from the corresponding ends of each scintillator. The output signals from the meantimers associated with the scintillators which are on line are sent to an AND circuit which produces the coincidence signal which is part of the hardware trigger and generates the event reference time.

The data words which compose the event processed by the HRS analyzer are the relevant times associated with the chambers and the scintillators and the analog signals associated with the two ends of $S_{2}$. The time data words are obtained by digitizing the time differences between stop and start signals sent to a set of LRS 2250T TDC(time-digital-converter) modules. The pulses coming from the two phototubes connected to $S_{2}$ are sent to a charge integrating ADC (analog-to-digital-converter)module.

The TDC's associated with the scintillators employ as start signal the time $\mathrm{T}_{0}$ defined by the 'event' signal, and as stop signal the time defined by the pulse coming from the up or down end of each counter. The average time $T_{a v e}=0.5 *$ $\left(T_{u p}+T_{\text {down }}\right)$ contains a term which is proportional to the arrival time of the event at the detector and a constant term which is determined by the delay elements introduced in the timing circuit, the transit time across the photomultiplier and the time taken by the light signals to truvel the whole scintillator length. The time difference $T_{\text {diff }}=T_{u p}-T_{\text {down }}$ contains a term which is proportional to the difference in transit time along the scintillator between the photons collected at the up end and the ones collected at the down end. Therefore it gives a rough indication of the vertical position of the event at the detector plane.

Each chamber plane, as mentioned before, has its sense wires connected to a delay line. The signals at the two ends of the delay line $\left(\mathrm{T}_{p}\right.$ and $\left.\mathrm{T}_{n}\right)$ provide two independent times from which the wire struck and the distance travelled by the electron pulse generated by the ionizing particle within the drift cell are 


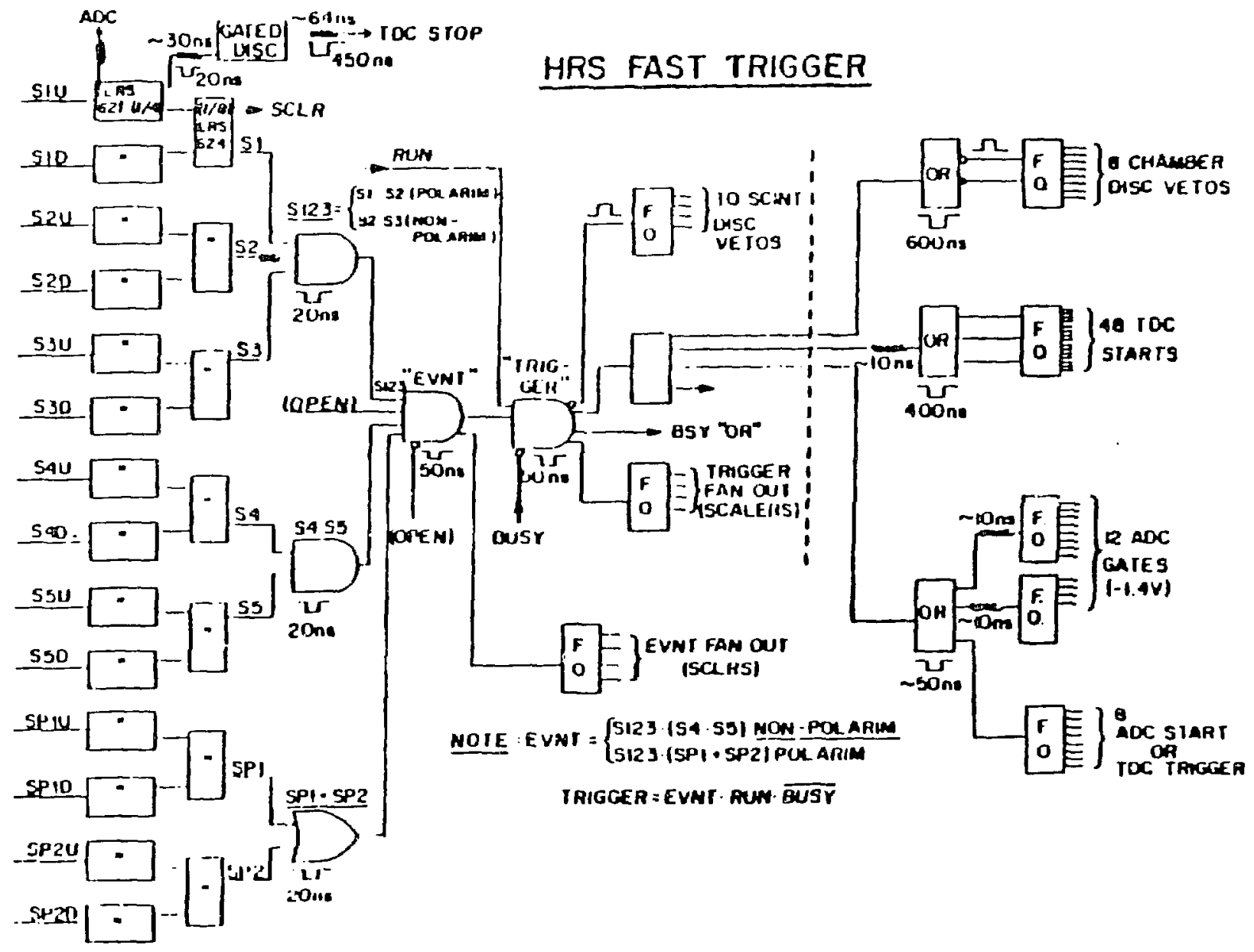

Figure 2.5: Block diagram of the HRS signal processor. 
reconstructed. At the HRS these two times are processed using two different reference times as $T_{\text {start }}$. The time $T_{p 0}$ employs the time $T_{0}$ as start and $T_{p}$ as stop, whereas the time $T_{n p}$ employs $T_{p}$ as start and $T_{n}$ as stop. The choice of $T_{n p}$ against the much larger $T_{n o}$ is determined by the requirement of making the time difference signal independent of the delay between the arrival of the particle at the chamber plane and the event reference time.

A technical feature of the TDCs used at the HRS is relevant to the discussion of the software reconstruction of the event which will be discussed in the next chapter. These TDCs are modified versions of the corresponding Lecroy ADCs $2250 \mathrm{~L}$, with an additional input stage composed of a constant current generator connected to an integrating capacitor during the time between the start and stop signal. The gate signal for the ADC stage (trigger input) is provided by the time $\mathrm{T}_{0}$. This produces an offset term in the digitized time which is proportional to the time difference $\left(T_{p}-T_{0}\right)$, corresponding to the time dependent pedestal characteristic of these ADCs. Therefore a correction must be applied for this effect as a first step of the software reconstruction of the chamber information.

Fig. 2.6 shows the 'slow gate' logic which is part of this electronics processor. It generates a set of flags about the beam conditions, computer status (BUSY signal), and data acquisition status (RUN signal). The selection of the kind of event to be processed is coordinated by the trigger module developed at LAMPF, tailored to the structure of the software employed for data acquistion.

An MBD (microprogrammed branch driver) constitutes the interface between the CAMAC crates and the on line computer which, during this experiment, was a PDP $11 / 45$. 


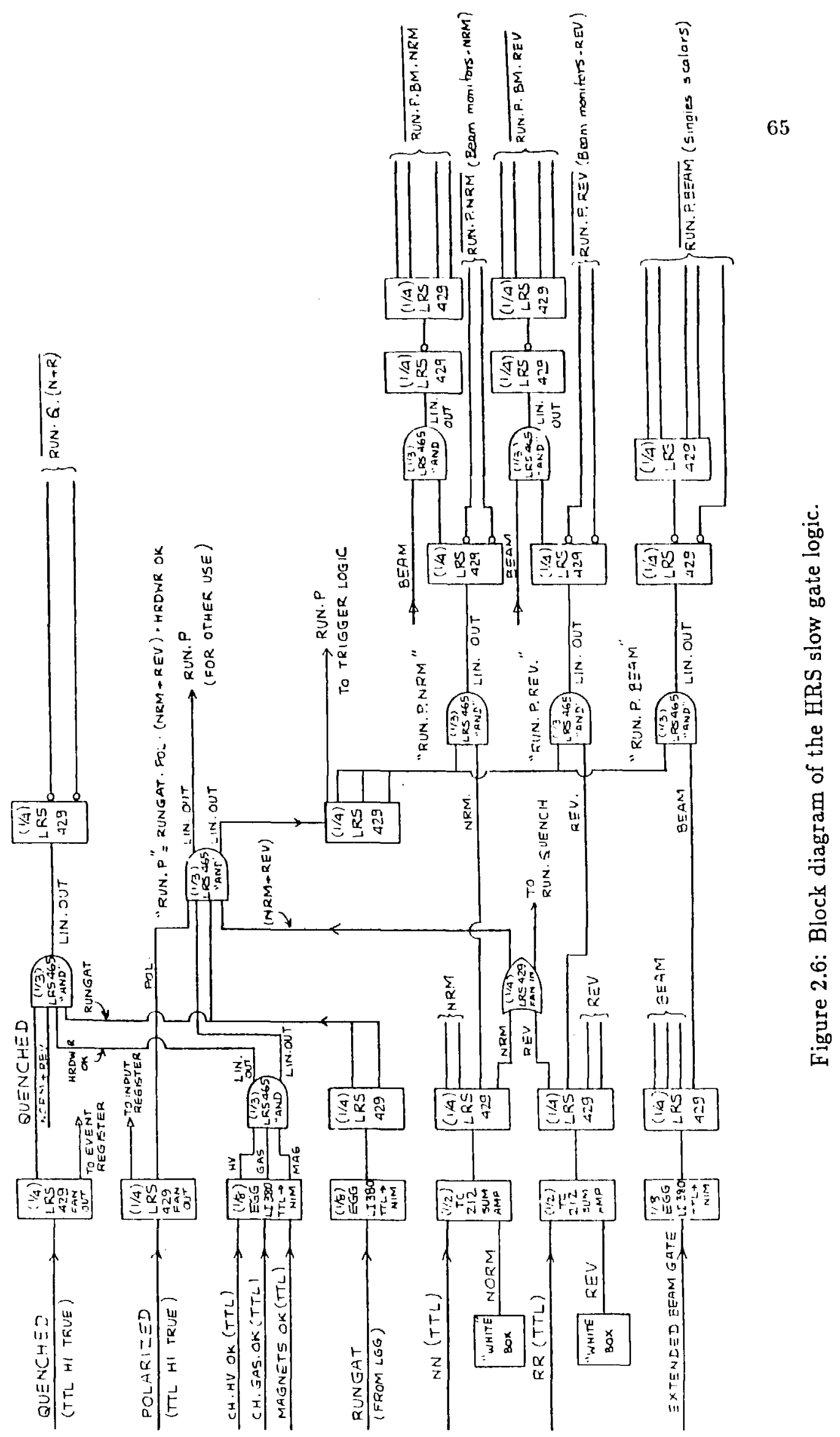




\subsection{The particle identification system.}

Three scintillator counters, $S_{2}, S_{4}$ and $S_{5}$ beyond the focal plane (see Fig. 2.4), were part of the detector configuration employed in this experiment: they had the purpose of providing the event trigger and the particle identification information. The variables which were employed for the latter purpose were the energy loss $\Delta E$ in the first scintillator and the time of flight between the first and last scintillator.

An investigation of the range of $\Delta E$ and time of flight TOF spanned by the detected particles was performed according to a simplified model. At the two angles correspording to the limits $\theta_{\min }$ and $\theta_{\max }$ of the angular acceptance of the spectrometer the range of kinetic energies spanned by all the particles w'ich are transmitted by the magnetic spectrometer was calculated. These particles with kinetic energies equal to $T_{\min }\left(\theta_{\max }\right)$ and $T_{\max }\left(\theta_{\min }\right)$ were then propagated through the three scintillators. This analysis was performed to identify the major sources of background and to obtain an estimate of the effectiveness of the experimental apparatus in particle detection and identification.

The critical aspect of the particle identification system is the difference ir the nature and kinematical parameters of the major sources of background. This is due to the different value of the parameter ' $\mathrm{P} / \mathrm{z}$ ' in the two cases: as all the 'candidate-background' particles have $\mathrm{z}=1$, their momentum wil! scale by a factor two going from the magnetic spectrometer setting suitable for ${ }^{3} \mathrm{He}$ to the one suitable for ${ }^{3} \mathrm{H}$. This is illustrated in Table 2.3, which shows the kinetic energies of the various background particles corresponding to the central momentum in the two cases.

Table 2.4 shows the range of time of flights and energy loss in $S_{2}$ for the 'signal' and 'background' particles in the two detection conditions.

It can be seen that in the case of ${ }^{3} \mathrm{H}$ detection the almost unique source of back-ground is consituted by the continuum deuterons, whereas in the case of ${ }^{3} \mathrm{He}$ three additional groups of particles (continuum p,d,t from the lower branch) fall 
Table 2.3: Kinetic energy of background particles corresponding to rigidity $\left(\mathrm{p}_{0} / \mathrm{z}\right)$ range $=1486.3-1523.9 \mathrm{MeV} / \mathrm{c} / \mathrm{z}$ for ${ }^{3} \mathrm{H}$ and $739.7-758.4 \mathrm{MeV} / \mathrm{c} / \mathrm{z}$ for ${ }^{3} \mathrm{He}$. (the asterisk denotes those particles which can not be produced by any reaction;

\begin{tabular}{|l|c|c|}
\hline & ${ }^{3} \mathrm{H}$ detection & ${ }^{3} \mathrm{He}$ detection \\
\cline { 2 - 3 } & $\mathrm{T}(\mathrm{MeV})$ & $\mathrm{T}(\mathrm{MeV})$ \\
\hline pion & $1492.6-1530.3^{*}$ & $613.2-631.6^{*}$ \\
proton & $819.4-851.3^{*}$ & $256.5-268.2$ \\
deuterons & $517.5-541.0$ & $139.5-147.5$ \\
tritons & $369.0-386.7$ & $95.8-100.6$ \\
${ }^{3} \mathrm{He}$ & $1281.0-1336.0^{*}$ & $365.8-383.4$ \\
\hline
\end{tabular}

Table 3.4: Time of flight and energy loss ranges for signal and background particles which enter the rigidity acceptance of the spectrometer.

\begin{tabular}{|l|l|l|l|l|}
\hline \multirow{2}{*}{} & \multicolumn{2}{|c|}{${ }^{3} \mathrm{H}$} & \multicolumn{2}{c|}{${ }^{3} \mathrm{He}$} \\
\cline { 2 - 5 } & TOF (ns) & $\Delta \mathrm{E}(\mathrm{MeV})$ & TOF (ns) & $\Delta \mathrm{E}(\mathrm{MeV})$ \\
\hline protons & - & - & $9.9-10.2$ & $3.3-4.8$ \\
deuterons & $9.9-10.8$ & $3.3-4.9$ & $17.3-17.9$ & $9.4-11.6$ \\
tritons & $13.1-13.5$ & $5.8-7.6$ & $28.1-30.2$ & $19.3-22.2$ \\
${ }^{3} \mathrm{He}$ He & & & $13.5-14.2$ & $24.8-28.3$ \\
\hline
\end{tabular}


within the acceptance of the magnetic spectrometer. However the former reaction is more critical, as the domains spanned in $\Delta E$ and TOF by tritons and deuterons are close to each other.

In the case of ${ }^{3} \mathrm{He}$ it is easier to achieve background separation as ${ }^{9} \mathrm{He}$ is well separated either in $\Delta E$ or in TOF from the background particles (see Table 2.4).

Table 2.4 clearly shows that, even if the detector system is the same in the two reactions, there are substantial differences for the two cases between the signals which have to be processed and in the spurious even shich have to rejected. It is mandatory to examine these issues carefully in the analysis of systematic errors to be attributed to the final results. 


\subsection{Monte Carlo simulation of detected particle piopagation through the spectrometer}

A Nonte Carlo code was written (see Appendix D) and a simulation of the experiment was done in order to investigate the correlations which exists between the momentum acceptance of the detector system and the angular spectra of ${ }^{3} \mathrm{H}$ and ${ }^{9} \mathrm{He}$. In principle, the range of scattering angles selected by the spectrometer should not be affected by the momentum of the particle detected. The momentum analysis is associaied with the vertical direction of the detector planes and the angular analysis with the horizontal one. The two acceptance boundaries are therefore determined by different physical dimensions and should be independent of each uther. However, because of tha large kinematic shift $(\mathrm{dT} / \mathrm{d} \theta \approx 10$ $\mathrm{MeV} / \mathrm{deg}$ ) in the reactions studied by us and the rather substantial momentum spread produced by the energy loss in the target, this edges of the angular spectrum are actually determined by the momentum range selected by the magnetic spectrometer. Fig. 2.7 illustrates this effect: the angular spectra. ${ }_{0}{ }^{9} \mathrm{H}$ and ${ }^{3} \mathrm{He}$ are compared with the one obtained during a calibration run for the reaction $\mathrm{D}\left(\mathrm{p}, \mathrm{p}^{\prime}\right)$ in the deep continuum ( at average excitation energy of $954 \mathrm{MeV}$ ), which had the main purpose of testing the uniformity in differential efficiency of the drift chambers.

The most problematic aspect which is apparent in this figure is the difference in the distortion induced by this effect in the two reactions to be compared. This is due to the difference in the width of the energy loss distribution for the reaction products caused primarily by the difference in their electric charge. As a consequence, there is a different detection efficiency for the two reactions to be compared.

The progiam discussed in this section was developed in order to obtain a quantitative evaluation of this effect. It investigates also the effects of multiple Coulomb scattering in the target and in the scintillator detectors and of the finite 


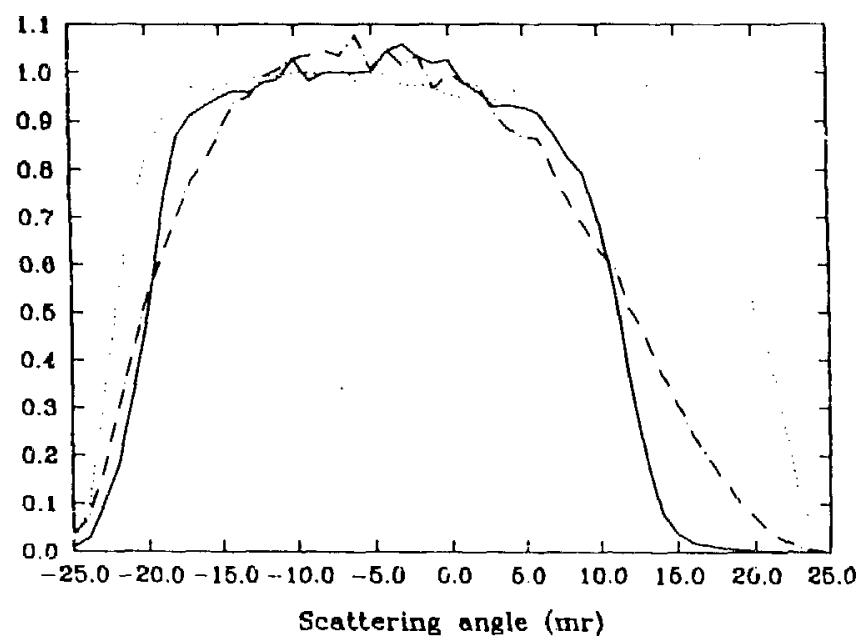

Figure 2.7: Experimental angular spectra for the reactions $\mathrm{D}\left(\mathrm{p},{ }^{3} \mathrm{H}\right) \pi^{+}$and $\mathrm{D}\left(\mathrm{p},{ }^{3} \mathrm{He}\right) \pi^{0}$ as compared with the angular acceptance of the HRS spectrometer for an inelastic reaction.

angular resolution associated with the trajectory reconstruction. Another major object of this simulation is the achievement of a deeper understanding of the shape of the distibutions of background events for the relevant spectra used to extract the ratio $\mathrm{R}$ and $\Delta A_{\mathrm{y} 0}$.

It is worthwile to examine the development of an event in the simulation in parallel with the corresponding situation in the data acquisition system in order to understand the model adopted and the approximations introduced in it. Fig. 2.8 illustrates the kinematical configuration and the relevant reference irames for the discussion which follows.

The initial state in a given experiment is characterized by the energy and the direction of he proton beam (particle $m_{1}$ ) and by the farget empioyed (particle $\mathrm{m}_{2}$ ). In the data acquisition system this information is given to the event processor as input parameters; no momentum reconstruction is performe 1 for the beam particles. This is motivated by the fact that the spectrometer was designed to operate in 'energy loss mode' described in Appendix 3 , and, in principle, the rele- 


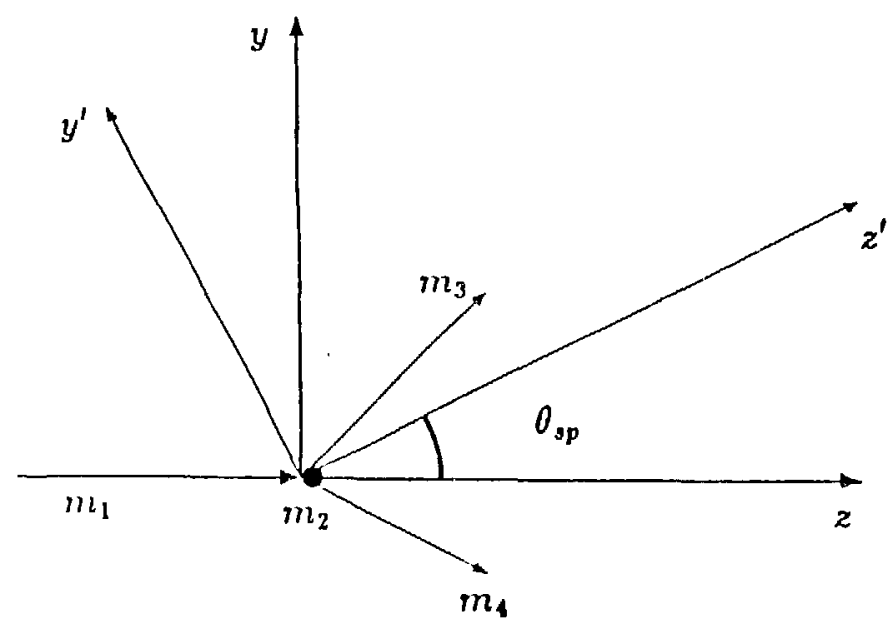

Figure 2.8: Coordinate system for the two-body reaction.

vant kinematical variables should not be affected by small variations of the beam energy around its nominal value. The effects of assuming a wrong centroid for the beam energy will be discussed later. In the simulation three input parameters are used to describe the beam kinetic energy: the 'nominal kinetic energy', $\mathrm{T}_{1}^{\text {nom }}$, corresponding to the value used in : e data analysis, the 'kinetic energy shift', $\left\langle\Delta T_{1}\right\rangle$, which represents the difference between the actual average energy and the nominal one, and the percent fractional momentum dispersion of the beam. For each event a 'irue' beam energy is generated according to the relationship:

$$
T_{1}^{\text {true }}=T_{1}^{\text {nom }}+\left\langle\Delta T_{1}\right\rangle+\Delta T_{1}^{f l u c t}
$$

where $\Delta T_{1}^{\text {fluct }}$ is a random variate uniformly distributed in the interval $\left|\Delta T_{1}^{\text {fluct }}\right| \leq$ $\Delta T_{1}^{\max } . \Delta T_{1}^{\max }$ is determined by the beam dispersion given as input parameter. Therefore it is possible to invessigate how the reconstructed variables are affected by the incorrect esimate of the beam energy $\left(\Delta T_{1} \neq 0\right)$ and by its energy spread in situations in which the energy loss compensation does not work. This is actually the case for the two reactions which are the object of our experiment.

The other major approximation performed in processing the data is to assume 
that the incident particle direction is always parallel to the $\hat{z}$ axis in Fig. 2.8. There are two main sources of deviation from this direction: the intrinsic angular divergence of the beam and the multiple Coulomb scattering suffered up to the interactic point. The former effect is not simulated, as no roliable information about it is avaibuble, whereas the latber effect i.s introduced in the program.

An interaction point with coordinales $\left(y_{t}, z_{t}\right)$ is generated for each eveat: $y_{t}$ represents tic horiznatial coordinate refeired $(1)$ the center of the target and $\mathbf{z}_{t}$ the coordinate along the beam direction referred to the entrance window of the tiargit.

The energy of the beam at the interaction point is corrected for the average encrgy loss and it:; flurtuations which took place in the thickness traversed $\Delta z_{b}$. The fluctuations aro generated arcording to a gaussian distribution, with a variance proportional to the square root of $\Delta z_{l}$. This functional dependence is suggested by Bohr's treatment of the fluctuations in the energy loss. However the coefficient employed is an empirical parameter consistent with that obtaine: with the Vavilov calculation of the FWIMM in this kinematical domain.

The vertical coordinate $x_{t}$ is not generated as it is not reconstructed in the event analyzer and therefore no observed correlation with this variable is available for comparison. The direction of the scattered particle trajectory is defined by the two angles $\theta_{t}$ and $\phi_{t}$, which define the orientation of its projections in the $x^{\prime} z^{\prime}$ and $y^{\prime} z^{\prime}$ planes respectively, in the spectrometer reference frame $\hat{x}^{\prime} \hat{y}^{\prime} \hat{z}^{\prime}$ shown in Fig. 2.8. The $\hat{z}^{\prime}$ axis lies in the horizontal plane along the central angle $\theta_{a p}$ of the spectrometer angular acceptance in this plane. In this crinncction it is relevant to observe that there can be an offset between the nominal spectrometer angle $\theta_{s p}^{\text {nom }}$ and the actual central angle $\theta_{s p}$ due to the error in the absolute angle calibration. Therefore the program has us input parameters two different spectrometer angles, in order to simulate the eff.cts of the offset describud above. The "true" $\theta_{s p}$ is used in calculating the true scattering angle, whereas the 'nominal' $\theta_{s p}$ is uscd to process the variabics to be compared with the experimental ones. 
The average energy loss of the scattered particle is calculated and also a simplified model of the fluctuations of energy loss is introduced, through a Gaussian straggling with a variance which is a function of the interaction point. Its functional form was determined in order to reproduce the dependence upon the interaction poiıt of the FWHM associated with the correct Vavilov distribution. In an earlier version of the program the fluctuations in energy loss were actually determined according to a set of Vavilov distributions. However, the complexity of the algorithm employed to generate random variates with this probability distributior required a discretization of the problem which introduced a worse approximation in the simulated spectrum of the kinetic energy of the scatiered particles than the solution finally adopted. The uncertainty in the scattering angle associated with multiple Coulomb scattering in the target is a!so introduced. Moreover a Gaussian straggling in the variables $\theta_{t}$ and $\phi_{t}$ is introduced to simulate the finite angular resolution of this detector system, as measured during the tune-up calibrations before the cycle in which this experiment was run.

In the HRS event processor algorithm, the transport properties of the spectrometer are determined empirically through a set of four functions which relate the coodinates at the front detector plare $\left(\mathrm{x}_{f}, \theta_{f}, \mathrm{y}_{f}, \phi_{f}\right)$ to the coordinates at the target $\left(\theta_{t}, \mathrm{y}_{t}, \phi_{t}\right)$ and the momanturi coordinate $\delta\left(=100 \cdot\left(\mathrm{p}-\mathrm{p}_{0}\right) / \mathrm{p}_{0}\right.$, where $\mathrm{p}$ is the reconstructed momentum and $\mathrm{p}_{0}$ is the central momentum of the magnetic spectrometer). This non-linear 'backward transport' system was inverted in order to propagate the scattered particles up to the first detector plane.

The geometrical characteristics of the detectors simulated have been given in Table 2.2. The particles are propagated though two sets of X-Y drift chambers and three scintillator planes. At each plane a test is performed on whether the transverse coordinates of the particle are within the geometrical acceptance of the detector plane. Each detector is considered ideal, i.e. its efficiency is assumed equal to one. Multiple Coulomb scattering and average energy loss effects in the scintillators are simulated. 
For the 'good' events, i.e. the ones which pass the acreptance tests at each detector plane, the relevant histograms to be compared th the experimental ones are constructed. The first set of histograms give information about the simulated spectra of the coordinates at the front plane $\left(v, \theta_{f}, y_{j}, \phi_{f}\right)$, allowing the comparison between the simulated and the Ieal spratwu distrit whon in the plane crossing the focal plane of the magnetie spertumenter. figs. 2.9, 2.10 give the comparison

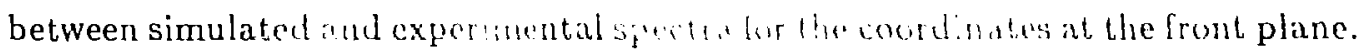
Figs. 2.11 gives the comparison between : mulated and experimental $x$ coordinate at the front plane for the detection of cm:tinumun protons. This spectrum has been singled out beanse the purpost of studying this ladution was to monitor the efficiency distribution 'n the focal plane along the dispersion direction $x$.

The two other releva"t spectra, from which the observabli's of our experiments $\sigma(\theta)$ and $A_{y 0}(\theta)$ are extracted, are the event spectrum as a function of the scattering angle $\theta_{s c}$ and the event spectrum as a function of the missing mass $M M$. The latter is the difference between the mass of the undetected particle calculated by the event analyzer and the theoretical value of $\mathrm{m}_{4}$ given to the analyzer as an input parameter. The experimental missing mass is a function of the reconstructed momentum of +1 . etected paricle $p_{3}$, the reconstructed scattering angle and the beam morneni. given as input parameter. Fur events which are associated with 'signal' reactions, the missing mass spectrum is represented by a peak, for which the centroid position is determined by the precision in the absolute values of the input parameters, and the width is determined by the spread in $p_{3}$ and $\theta_{s c}$ produced by energy loss effects and the spatial resolution of the drift chambers. Some of these effects are simulated reasonably well. This is illustrated by the comparison of the missing mass spectra from the experiment and the Monte Carlo program. Fig. 2.12 illustrates the comparison for the $\mathrm{p}+\mathrm{L} \longrightarrow{ }^{3} \mathrm{He}+\pi^{0}$ and $\mathrm{p}+\mathrm{D} \longrightarrow{ }^{\mathrm{s}} \mathrm{H}+\pi^{+}$reactions and for the reaction $\mathrm{d}\left(\mathrm{p}, \mathrm{p}^{\prime}\right) \mathrm{X}$ in the deep continuum. Fig. 2.13 illustrates the cumparison for elastic scattering runs performed with various targets for efficiency and encrgy monitoring. 

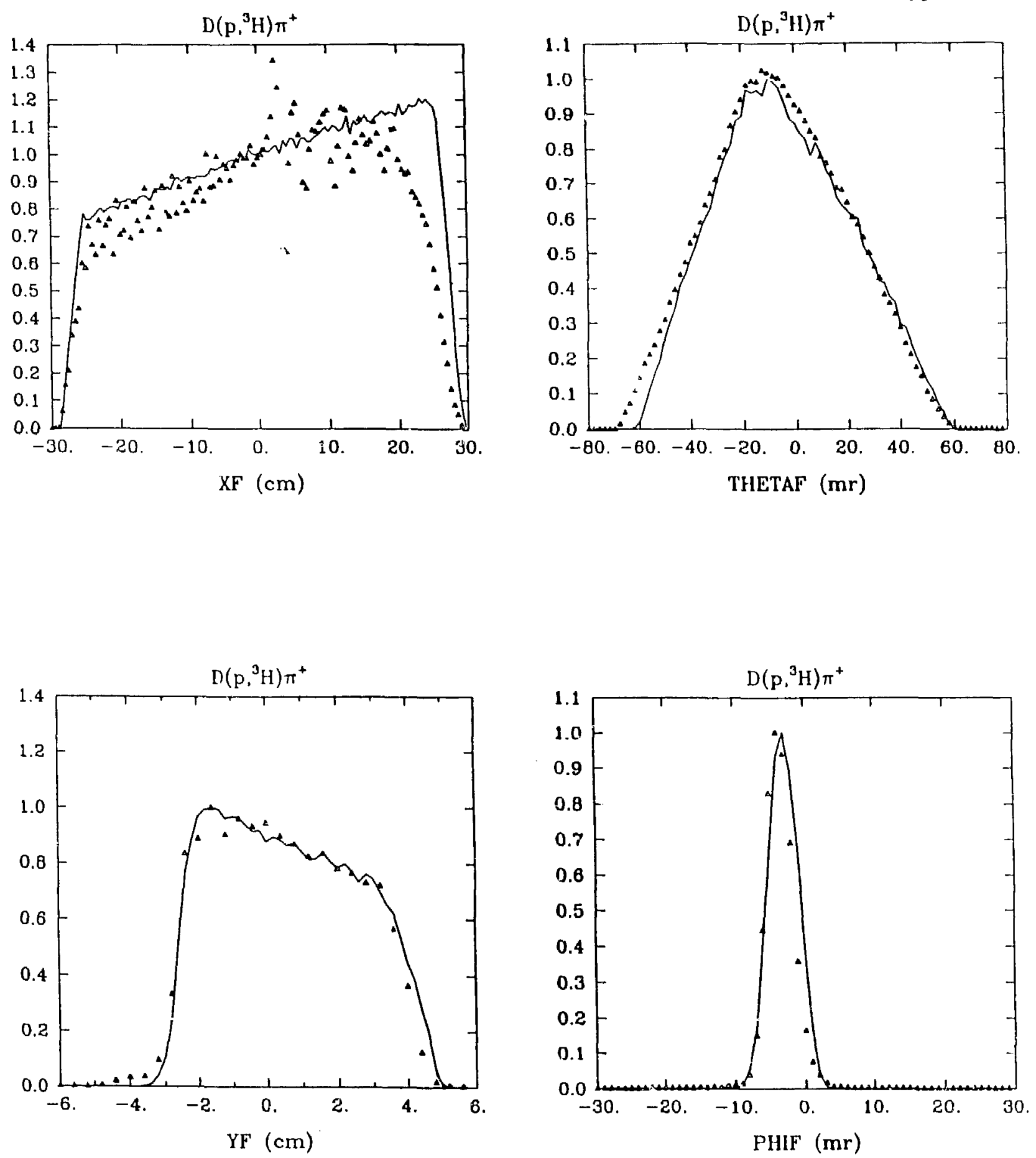

Figure 2.9: Coordinates $\left(\mathrm{x}_{f}, \theta_{f}, \mathrm{y}_{f}, \phi_{f}\right)$ at the front plane for the reaction $\mathrm{p}+\mathrm{d}$ $\rightarrow \rightarrow^{3} \mathrm{H}+\pi^{+}$. 

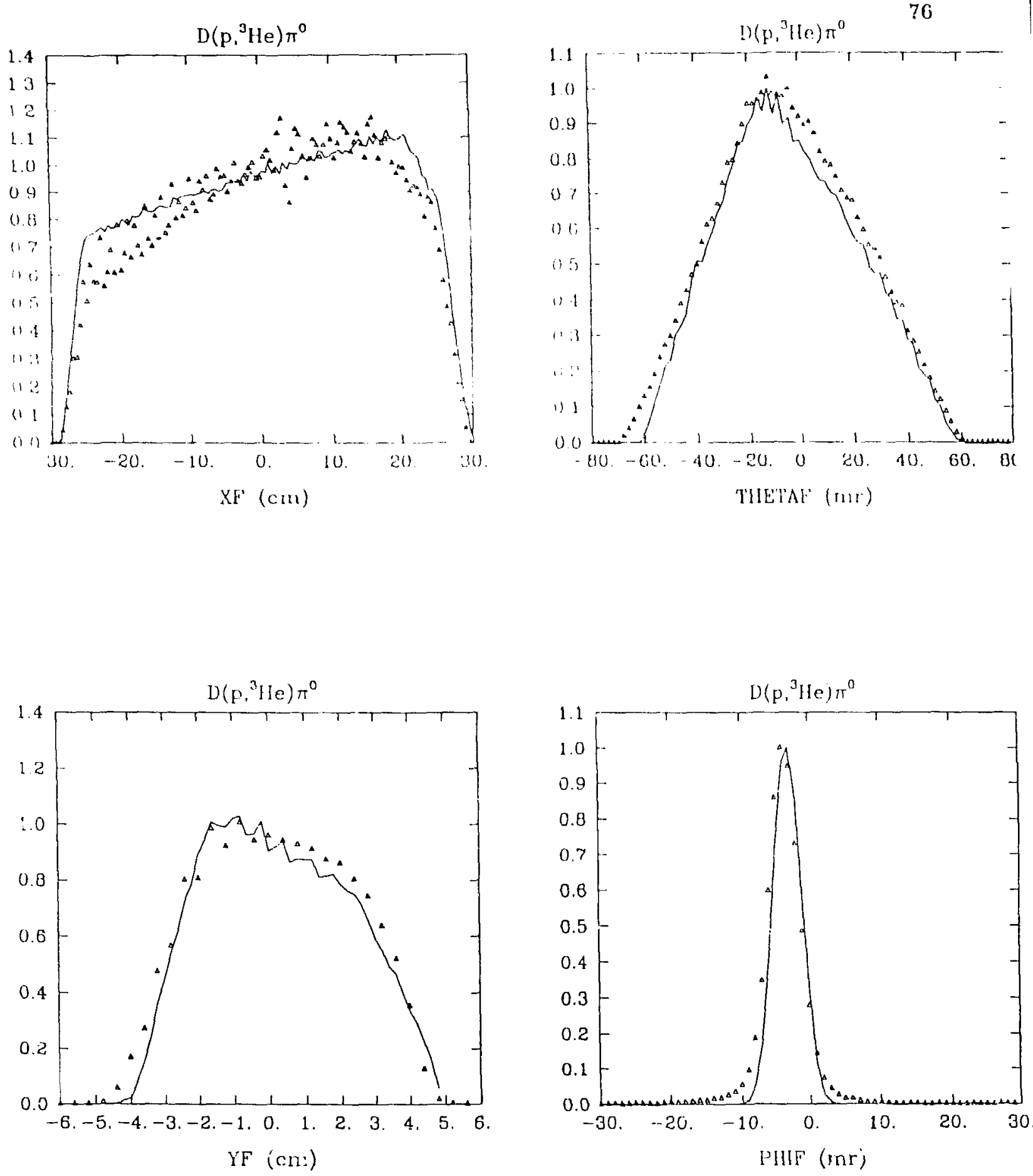

Figure 2.10: Coordinates $\left(\mathrm{x}_{f}, 0_{f}, \mathrm{y}_{f}, \phi_{f}\right)$ at the front plane for the reaction $\mathrm{p}+\mathrm{d}$ $\rightarrow^{3} \mathrm{He}+\pi^{0}$. 


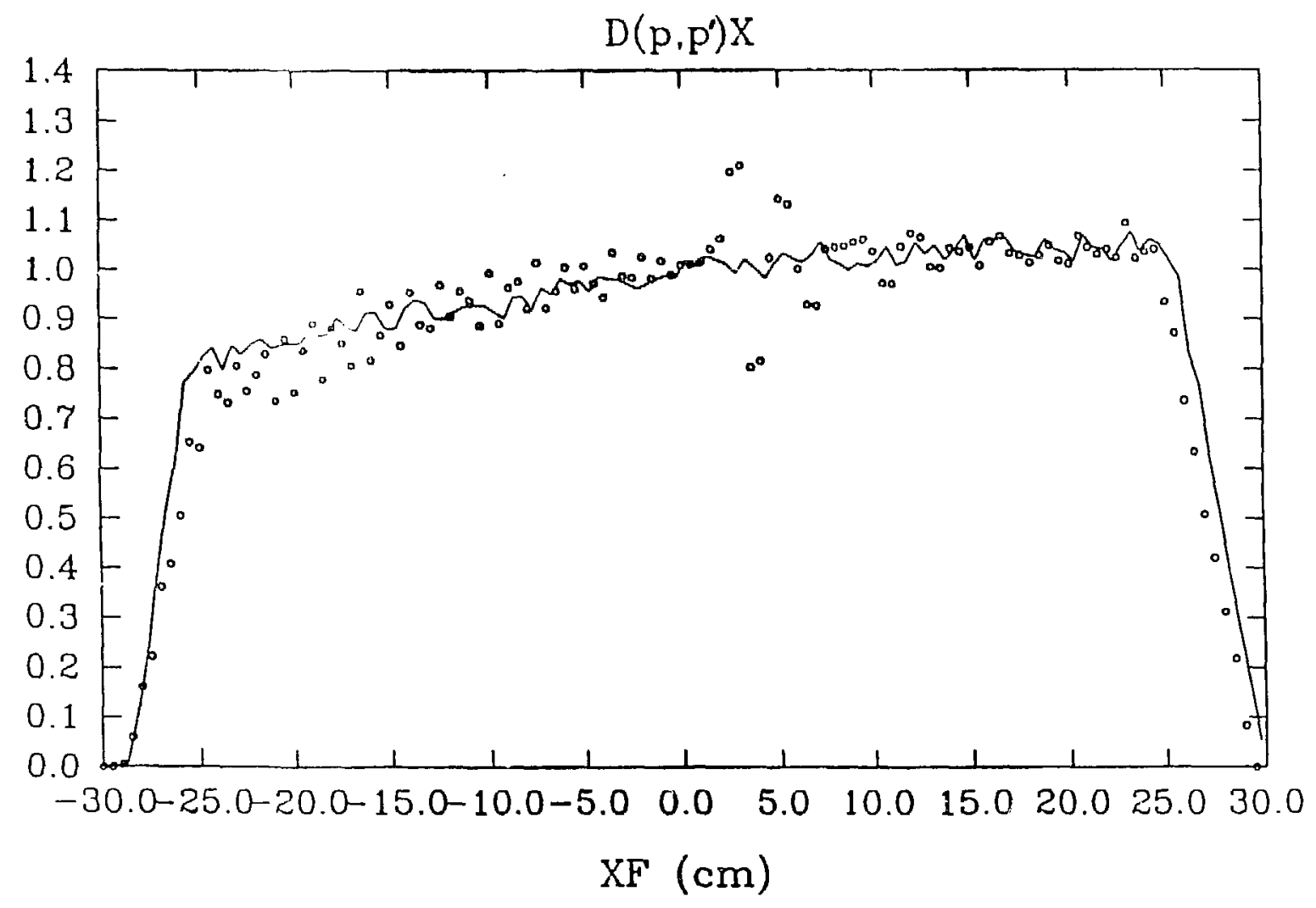

Figure 2.11: Coordinate $x_{j}$ at the front plane for the reaction $p+d \rightarrow p^{\prime}+X$. 

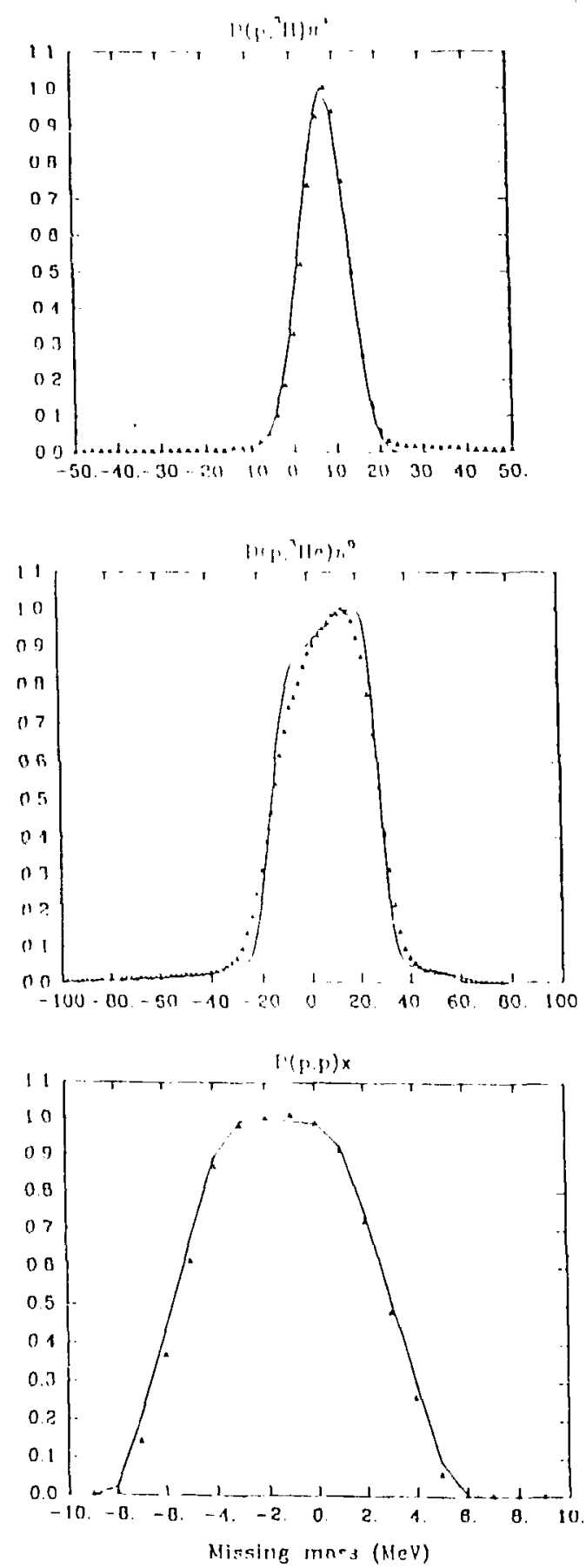

Figure 2.12: Normalized missing mess spectra from Monte Carlo simulation (continuous line) and from experimental data for the three rcactions $d\left(p,{ }^{3} \mathrm{H}\right), \mathrm{d}\left(\mathrm{p},{ }^{3} \mathrm{He}\right)$ and $d\left(p, p^{\prime}\right)$. 

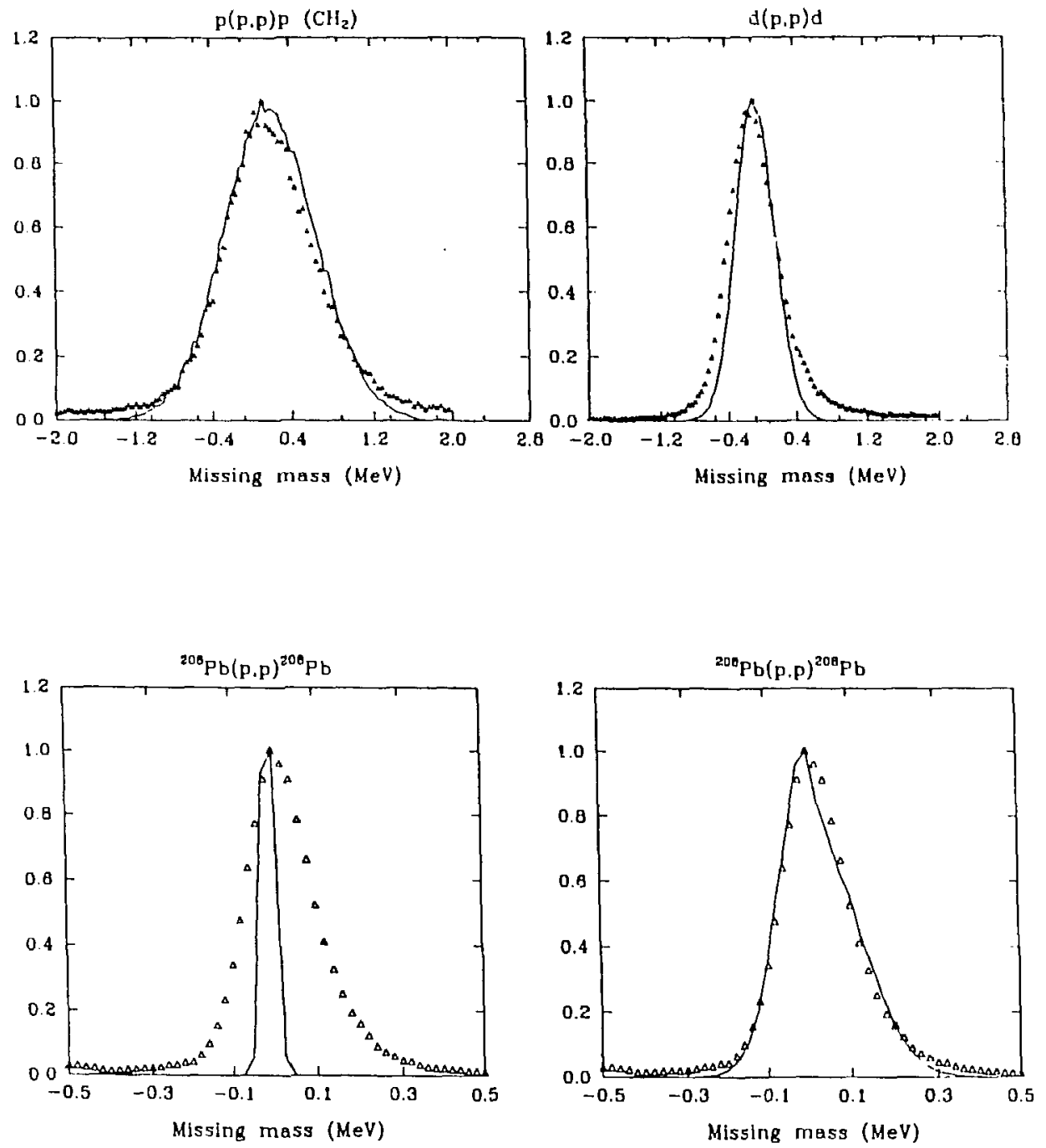

Figure 2.13: Normalized missing mass spectra from Monte Carlo simulation (continuous linej and from experimental data for elastic scattering from protons, deuterons and ${ }^{208} \mathrm{~Pb}$. For ${ }^{208} \mathrm{~Pb}$ the curve in the figure on the left is without taking into account any intrinsic momentum resolution contribution, and that in the figure on the right is with inclusion of $0.018 \%$ intrinsic momentum resolution. 
The only missing rnass width which is not reproduced correctly is the one associated with elastic scattering from ${ }^{208} \mathrm{~Pb}$ (see Fig. 2.13). In this case the experimental width is determined by the intrinsic momentum resolution of the spectrometer, which is determined by its optical properties (non-linearities, aberrations etc.). An empirical Gaussian spread of the variable $\delta$ has been introduced in the program in order to sinulate this effect. Its variance has been taken from the experimental value determined by the $\delta$ spectrum for elastic scattering for ${ }^{208} \mathrm{~Pb}$. However it turns out that this contribution to the missing mass width is already negligible for the spectra corresponding to elastic scattering from deuterium. Overall, the widths of the milsing mass distributions are well reproduced. Some discrepancy exists between the centroids of the missing mass spectra obtained from the simulation and the experimental ones. The relative shifts which have been introduced in order to superimpose the experimental and simulated peak are within the error associated with the experimental centroids $(\approx 50 \mathrm{C} \mathrm{KeV}$ for elastic scattering and $\approx 2 \mathrm{MeV}$ for ${ }^{3} \mathrm{H}$.)

The distributions of events with respect to the scattering angle are well reproduced, as shown in Fig. 2.14.

Our ability to simulate the missing mass spectra for these 'signal' events gives us confidence in the simulation for the missing mass spectra for the 'background' events generated on the basis of simple, plausible hypotheses about their sources. The steps taken in the generation of 'background' events are the same as a'scussed before. The hypothesis we use is that the background events are genera'ed as continuum production from impurities in the target (mostly the material of the $\mathrm{LD}_{2}$ container, aluminized mylar, etc.).

Table 2.5 is a summary of the input parampters employed in the simulation of the two reactions $\mathrm{d}\left(\mathrm{p},{ }^{3} \mathrm{H}\right) \pi^{+}$and $\mathrm{d}\left(\mathrm{p},{ }^{3} \mathrm{He}\right) \pi^{0}$.

Table 2.6 gives the percentage event rejection by the various ditector planes for the specified experimental conditions.

Table 2.7 gives the percent efficiency for two arıgular regions over which 

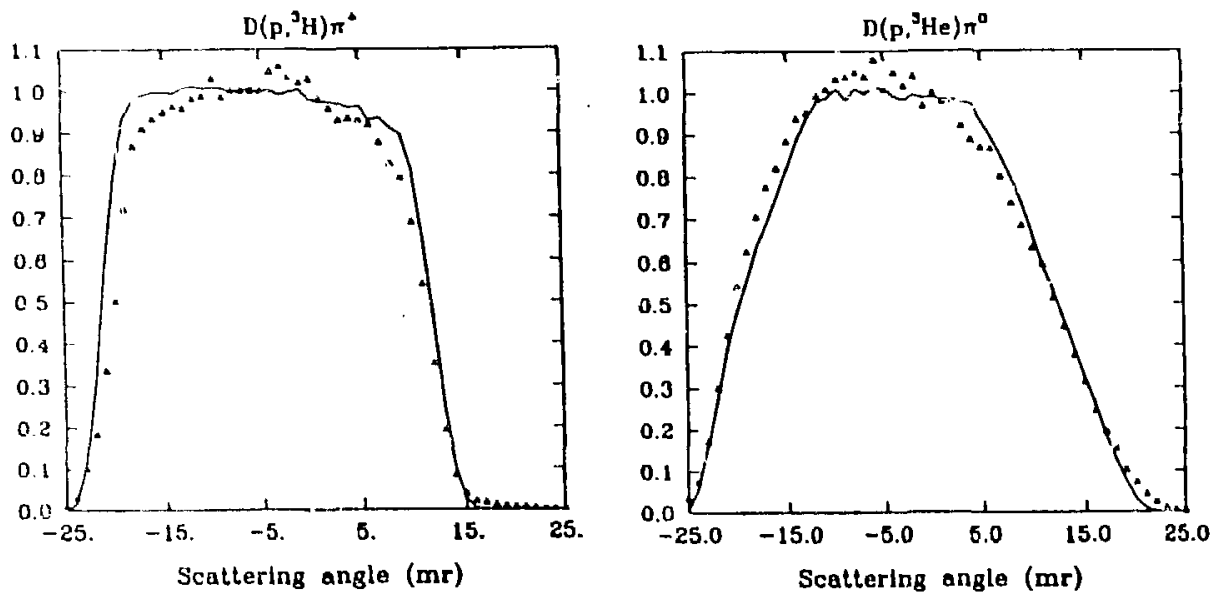

Figure 2.14: iVormalized scattering angle spertra from the Monte Carlo simulation and experimental data.

Table 2.5: Input parameters for the Mionte Carlo simulation of the reactions $\mathrm{d}\left(\mathrm{p},{ }^{3} \mathrm{H}\right) \pi^{+}$and $\mathrm{d}\left(\mathrm{p},{ }^{3} \mathrm{He}\right) \pi^{0}$.

\begin{tabular}{|l|l|l|}
\hline & ${ }^{3} \mathrm{H}^{+}$ & ${ }^{3} \mathrm{He}^{++}$ \\
\hline $\mathrm{T}_{1}^{\text {nom }}$ & $730 . \mathrm{MeV}$ & $730 . \mathrm{MeV}$ \\
$\left\langle\Delta T_{1}\right\rangle$ & $0.13 \mathrm{MeV}$ & $0.13 \mathrm{MeV}$ \\
$\delta \mathrm{p}^{\text {in }} / \mathrm{p}^{\text {in }}(\%)$ & \pm .06 & \pm .06 \\
target thickness & $0.390 \mathrm{~g} / \mathrm{cm}^{2}$ & $0.390 \mathrm{~g} / \mathrm{cm}^{2}$ \\
HRS central $\left.\mathrm{m}_{\mathrm{t}}\right) \mathrm{m}$. & $1501.36 \mathrm{MeV} / \mathrm{c}$ & $747.153 \mathrm{MeV} / \mathrm{c}$ \\
$\theta_{s, p}^{\text {nom }}$ & $12.000 \mathrm{deg}$ & $12.000 \mathrm{deg}$ \\
$\theta_{s p}^{\text {true }}$ & $12.15 \mathrm{deg}$ & $12.15 \mathrm{deg}$ \\
$\sigma\left(\phi_{t}\right)$ & $1.0 \mathrm{mr}$ & $1.0 \mathrm{mr}$ \\
\hline
\end{tabular}


Table 2.6: Monte Carlo results for detector acceptance in of the particles which arrive at a detector.

\begin{tabular}{|l|c|c|c|}
\hline detector plane & ${ }^{3} \mathrm{H}$ & ${ }^{3} \mathrm{He}$ & Ratio \\
\hline $\mathrm{C} 1$ & 78.01 & 74.42 & $1.05 \mathrm{C}$ \\
$\mathrm{C} 1 \cdot \mathrm{C} 2$ & 75.27 & 71.63 & 1.051 \\
$\mathrm{C} 1 \cdot \mathrm{C} 2 \cdot \mathrm{S} 2$ & 72.75 & 71.63 & 1.016 \\
$\mathrm{C} 1 \cdot \mathrm{C} 2 \cdot \mathrm{S} 2 \cdot \mathrm{S} 4$ & 72.75 & 71.63 & 1.016 \\
$\mathrm{C} 1 \cdot \mathrm{C} 2 \cdot \mathrm{S} 2 \cdot \mathrm{S} 4 \cdot \mathrm{S} 5$ & 72.65 & 71.63 & 1.016 \\
\hline
\end{tabular}

Table 2.7: Integral efficiency for the two angular gates cmpioyed in the analysis from the Monte Carlo simulation. (Angles are referred to the spectrometer angle $\theta_{\text {a.) }}$

\begin{tabular}{|c|c|c|c|c|c|}
\hline & \multicolumn{3}{|c|}{${ }^{3} \mathrm{H}$} & & \multirow{2}{*}{ Ratio ${ }^{3} \mathrm{H} /{ }^{3} \mathrm{He}$} \\
\cline { 1 - 5 } Gate & $\Delta \theta(\mathrm{mr})$ & Efficiency (\%) & $\Delta \theta(\mathrm{mr})$ & Efficiency $(\%)$ & \\
\hline$\Delta \theta_{1}$ & $-15.8-+8.2$ & $97.8 \pm .14$ & $-15.0-+9.0$ & $94.5 \pm .14$ & $1.035 \pm 0.20$ \\
$\Delta \theta_{2}$ & $-13.8-+2.2$ & $98.9 \pm .14$ & $-13.0-+3.0$ & $97.7 \pm .17$ & $1.012 \pm 0.22$ \\
\hline
\end{tabular}

the measured observables were integrated. The intervals used for ${ }^{3} \mathrm{H}$ are slightly shifted with respect to the ones employed for ${ }^{3} \mathrm{He}$ in order fur their centroids to correspond to the same pion angle in the center of mass systerr. The reasons for this choice will become clearer when the method of analysis is discussed. 


\section{Chapter 3}

\section{Data analysis and results.}

The presentation of the results obtained in this experiment will be preceeded by a discussion of the relevant software employed in processing the information obtained from the data acquisition system. This gives an understanding of the various steps involved and of the sources of uncertainty associated with them. Next, a discussion of the method of analysis employed will be given and the results for the observables measured will be presented. Finally the major sources of systematic errors will be analyzed.

\subsection{The analysis and calibration software.}

There are two main sof tware packages which are employed to handle the data acquisition process and the off-line analysis for experiments performed at the HRS facility.

The first, generally known as ' $Q$ system', is a set of programs and subroutines for data handling and manipulation. They provide the ability of handling the transfer of data words from the Camac modules in the electronics processor to a storage device (typically a magnetic tape), of imposing software cuts on specifir. data words and of calculating the relevant derived quantities. The latter function requires the input information of a set of parameters which characterize the experimental conditions. 'They are stored in a large cornmon block shared by the subroutines which manipulate the data. Some of them are obtained in calibration 
runs (e.g. chämber parameters), other are entered interactively at the beginning of a run (typically this is the case for kinematics parameters relative to the experiment performed). Detailed information about the various components of this system can be found in ref. ${ }^{88}$

A second set of programs, developed by the MP10 staff ${ }^{89}$, is tailored to the data acquisition system present in the HRS and EPICS facilities. The core of this software package is the 'HRS event analyzer'. The version which is used during data acquisition contains a description of the CAMAC modules which interact with the computer and the set of steps which need to be performed upon the arrival of a specific trigger signal. A version modified in its input-output operations is used for the analysis of the data. Other programs have been developed to perform calibrations, kinematics calculations and rata handling. More detailed information about these can be found in reference (89).

\subsection{The HRS event reconstruction.}

Each event which is stored on tape consists of readings of the TDCs associated with the drift chambers and the scintillaturs and the ADCs associated with scintillator $S_{2}$. The information from the scintillators is used to generate the 'particle identification test' described in the previous chapter. The time data words from the drift chambers allow the reconstruction of the kinematic variables 'true scattering angle' $\theta_{s c}$ and missing mass MM, which have been defined in the section devoted to the Monte Carlo simulation.

The missing mass spectrum allows the identification of the reaction of interest against competing ones which fall within the acceptance of the magnetic spectrometer and satisfy the particle identification test. It can also be employed to obtain the yield from which the differential cross section or the analyzing power are calculated. Alternatively, these observables can be obtained from the integration of the scattering angle spectrum in selected intervals contained within the angular acceptance. The difference between the two approaches will be discussed 
in a later section.

The first step in the reconstruction of the kinematic variables for the event processed involves the calculation of the position and orientation of the particle detected at the median plane of the first set of drift chambers. Fig. 3.1 gives a schematic representation of the steps taken to accomplish this task. These operations are performed by processing simultaneously the information from each pair of adjacent chamber planes.

The step which deserves more careful analysis is the interpolation which determines the final value of the 'corrected extrapolated position at the mid-plane'. It involves the definition of variables which are crucial in the characterization of the chamber performance. The geometry pertinent to this discussion is given in Fig. 3.2.

A crude determination of the orientation of the event in the $x^{\prime}-z^{\prime}$ plane (or $y^{\prime}-z^{\prime}$ plane), characterized by the angle $\theta^{\prime}\left(\phi^{\prime}\right)$, is needed to extrapolate the position at the mid-plane between the two adjacent chambers. Subsequently, four extrapolated positions at plane $x_{i}^{*}$ are calculated from the information associated to the two corresponding chamber planes.

$$
\begin{aligned}
& x_{c}(I)=x_{t}(1)+x_{d r}(1)+c_{0} \\
& x_{c}(I I)=x_{\imath}(1)-x_{d r}(1)+c_{0} \\
& x_{c}(I I I)=x_{t}(2)+x_{d r}(2)-c_{0} \\
& x_{c}(I V)=x_{t}(2)-x_{d r}(2)-c_{0}
\end{aligned}
$$

where:

$$
\begin{aligned}
& c_{0}=\tan \left(\theta^{\prime}\right) \cdot \Delta z^{\prime} / 2 \\
& x_{t}(i)=\text { truncated position at plane } \mathrm{i} \\
& x_{d r}(i)=\text { drift distance at plane } \mathrm{i}
\end{aligned}
$$




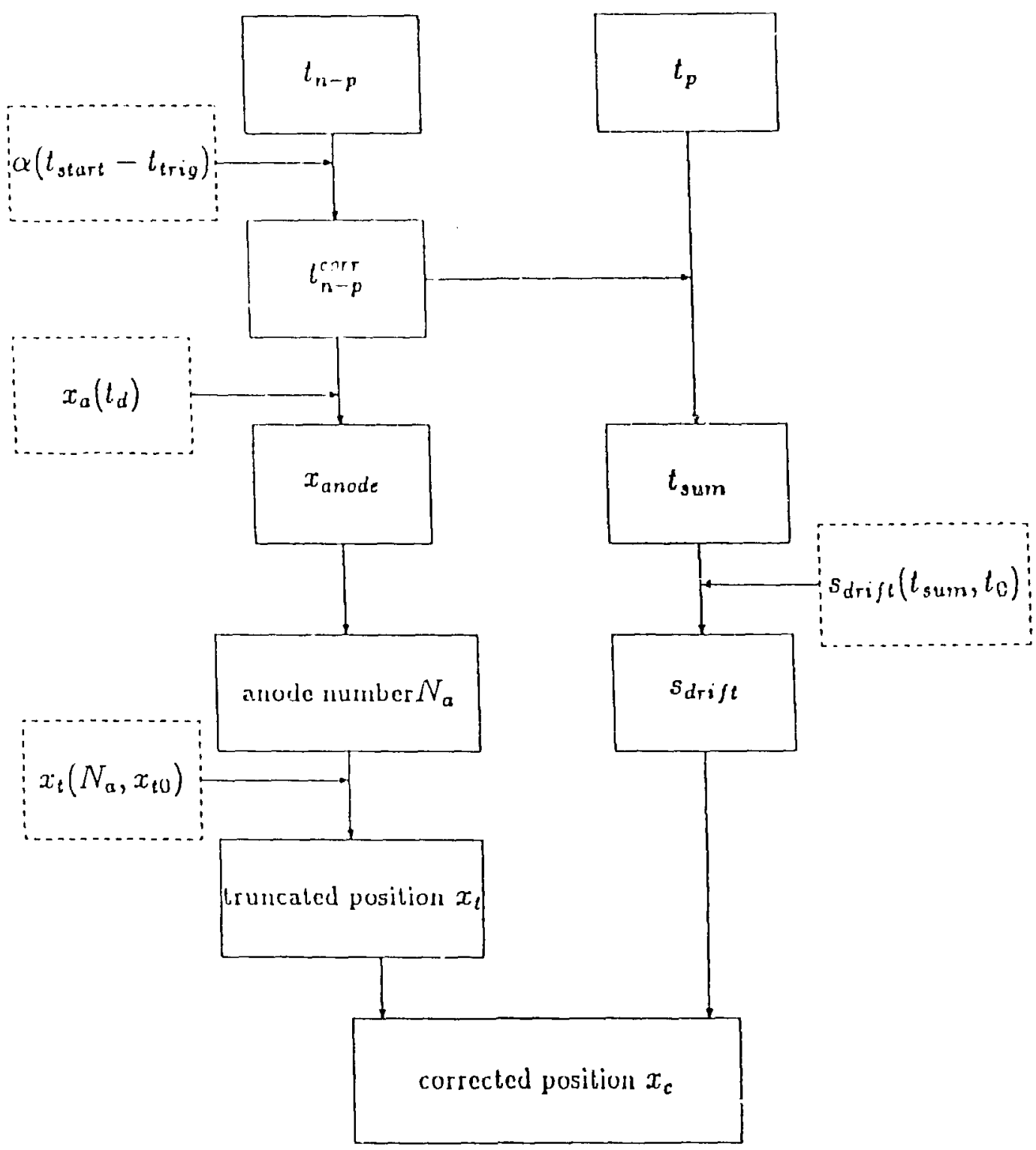

Figure 3.1: Flow-diagram of the event reconstruction performed by the Illes event processor. 


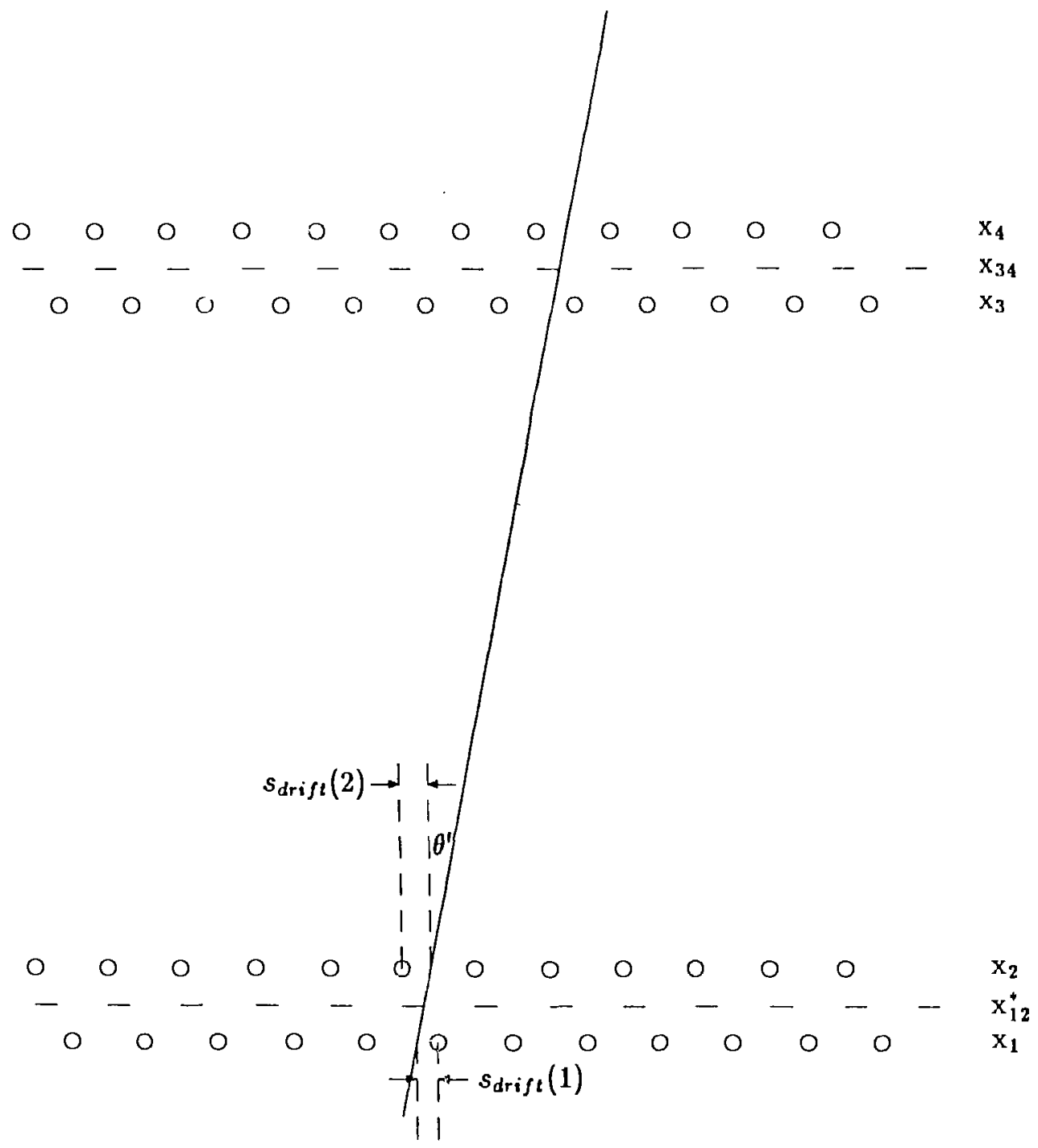

Figure 3.2: Geometry for the determination of the corrected extrapolated position of a particle track through the $\mathrm{x}$ drilt cliambers. 
Subsequently the four possible differences $\Delta x_{i j}, \mid x_{c}(\mathrm{I})-\mathrm{x}_{c}(\mathrm{III}), \mathrm{x}_{c}(\mathrm{I})-\mathrm{x}_{c}(\mathrm{IV}), \mathrm{x}_{c}(\mathrm{II})-$ $x_{c}(I I I), x_{c}(I I)-x_{c}(I V)$, between the first two and the last two elements of the array $x_{c}$ are constructed. Ideally, one of these differences should be equal to zero, as it corresponds to the combination of the truncated position with the drift distance performed with the choice of sign corresponding to the correct track orientation. This implies that the choice of the combination of signs which corresponds to the difference with minimum absolute value $\Delta x_{i j}^{m i n}$ should be the correct one. The 'corrected extrapolated position' at $x_{i}^{*}$ is the average between the extrapolated positions from the two adjacent planes chosen by this criterium. The discussion of this step in the algorithm for event recnnstruction has been carried out in detail as the variables $\Delta x_{i j}^{m i n}$ play a crucial role in the software test of correct wire identification which was particularly critical for this experiment. These considerations will be developed in the next section.

The coordinates at the front plane $\left(x_{f}, \theta_{f}, y_{f}, \phi_{f}\right)$ are obtained though the relationships:

$$
\begin{gathered}
x_{f}=x_{i}^{*} \\
\theta_{f}=\left(x_{2}^{*}-x_{1}^{*}\right) / \Delta z \\
y_{f}=y_{i}^{*} \\
\phi_{f}=\left(y_{2}^{*}-y_{1}^{*}\right) / \Delta z
\end{gathered}
$$

where:

$x_{i}^{*}$ and $y_{i}^{*}$ are the 'corrected extrapolated positions' at the mid-planes defined previously.

$\Delta z$ is the separation between corresponding intermediate planes in the front and rear set of chambers.

The coordinates $\left(x_{f}, \theta_{f}, y_{f}, \phi_{f}\right)$ are used to calculate the variables $\left(y_{t}, \phi_{t}, \theta_{t}, \delta\right)$, which characterize the scattering event. The variables $\left(y_{t}, \phi_{t}, \theta_{t}\right)$ give the relevant information about the initial trajectory of the detected particle.. The variable $y_{t}$ corresponds to the horizontal displacement with respect to the target center and 
$\phi_{t}, 0_{t}$ correspond to the angular divergences with respect to the spectrometer central ray, in the corizontal and vertical plane respectively. The vertical coordinate at target, $x_{t}$, which is proportional to the dispersion of the beam, is unobserved. The variable $\delta$ represents the percent deviation $100 \cdot\left(p_{3}-p_{0}\right) / p_{0}$, where $p_{0}$ is the spectrometer central momentum. The relationships which are used to calculate the variables $\left(y_{t}, \phi_{t}, \theta_{t}, \delta\right)$ from $\left(x_{f}, \theta_{f}, y_{f}, \phi_{f}\right)$ correspond to a nonlinear generalization of the inverse 'transport matrix' of the magnetic spectrometer. They are four second-order polynomials. Their coefficents are determined empirically in calibration runs. In the experiment performed a mapping of the momentum of the detected particle across the whole focal plane was of interest. Therefore the calibration determining the functional dependence $\delta\left(x_{f}, \theta_{f}, y_{f}, \phi_{f}\right)$ was repeated. The method employed and the results obtained will be discussed in the next seciion.

The knowledge of the momentum and orientation of the scattered particle allows the reconstruction of $\theta_{s c}$ and MM. These variables have been defined in the section describing the Monte Carlo simulation performed. The missing mass variable $M M$ is a function of the reconstructed momentum $p_{3}$ and scattering angle $\theta_{s c}$ and of the input parameters $T_{1}$ (nominal beam energy), $\theta_{s}$ (spectrometer angle) and the parameters describing the reaction under investigation.

Each of the reconstructed datd words discussed in this section is available for histogram display, if the spectral distribution of events relative to it is judged of interest. Selected regions of the range of values spanned by a data word can be singled out for the generation of software cuts employed to process the events. 


\subsection{Calibrations.}

The calibrations needed to process the time information coming from the drift chambers and to relate the coordinates at the front plane to the ones at the target are ubtained during the tune-up runs performed at the beginning of each cycle of experiments. However some of these functions are sensitive to parameters specific of the experiment performed (e.g. magnetic spectrometer field setting, chamber operating voltage etc.). For this experiment new drift time calibrations $s_{\text {drifit }}\left(t_{s u m}, t_{0}\right)$ and $\delta\left(x_{f}, \theta_{f}, y_{f}, \phi_{f}\right)$ calibration were done.

The drift time calibration associates a drift distance to each drift time bin. It is sensitive to the detailed shape of the electric field within the drift cell. In these chambers the boundaries of the drift cells are defined by field-shaping cathode wires located at a distance equal to $\mathrm{w} / 2$ between two adjacent anode wires. Near the anodes, there is a region of high field (with a space dependence $\approx 1 / \mathrm{r}$ ) where the multiplication takes place. When the chambers are illuminated uniformly across their active region, the drift distance spectrum should be flat. However, when the wrong weighting functions are used, these spectra develop spikes corresponding to either of the two boundary regions discussed above. During our experiments the drift chambers were sperated at two different voltages, $2000 \mathrm{~V}$ for ${ }^{3} \mathrm{H}$ and $1800 \mathrm{~V}$ for ${ }^{3} \mathrm{He}$. As a consequence, it was necessary to perform individual calibrations for ${ }^{3} \mathrm{H}$ and ${ }^{3} \mathrm{He}$. A task (named DRT), which is part for the MP10 software package discussed above, allows the determination of $s_{d r i f t}\left(t_{s u m}, t_{0}\right)$. Two steps are involved in this procedure. The first one determines the offset term $t_{0}$. For correct values of $t_{0}$, the drift time spectra orresponding to the different chamber planes should exhibit no relative displacement. Fig. 3.3 shows the drift time spectra for the eight chambers X1-X4, Y1-Y4 after the offset calibration performed for ${ }^{3} \mathrm{HL}$ and ${ }^{3} \mathrm{He}$ respectively. Next the driít distance to drift time dependence is obtained as an array which contains the weighting function which relates each drift time bin $t_{d r i f t}^{i}$ to the corresponding drift distance $s_{d r i f t}^{i}$. These arrays were 
obtained for each chamber plane separately. The drift distance spectra obtained after the calibration was performed are given for each chamber plane and for the two reactions in Figs. 3.4-3.7.

The other calibration performed was the one which determines the coeflicients of the polynomial function $\delta\left(x_{f}, \theta_{f}, y_{f}, \phi_{f}\right)$. The standard procedure developed at HRS for this purpose was followed. It involves taking data for protons elastically scattered from a heavy target for various settings of the spectrometer magnetic field so that the elastic peak moves across the dispersion plane. In the analysis of these runs some ray files are written. Each ray contains the valuc of $\delta$ as input parameter for each run, the coordinates $\left(x_{f}, \theta_{f}, y_{f}, \phi_{f}\right)$ and the reconstructed scattering angle $\theta_{s c}$ for the event processed. The latter variable is used to correct for the kinematic shift associated with scattering events which took place at an angle $\theta_{s c}$ different from the spectrometer angle $\theta_{s p}$. The ray file is written by the task FPO running simultaneously with the analyzer. Another task (COF) reads the ray file and a file describing the structure of the polynomial form expected for $\delta$ and determines the coefficient of each term by a minimization of the chi-square function $\left(\chi^{2}\right)$. Fig. 3.8 shows the missing mass spectrum for elastic scattering from ${ }^{208} \mathrm{~Pb}$ analyzed with the $\delta\left(x_{f}, \theta_{f}, y_{f}, \phi_{f}\right)$ obtained from the calibration performed. Fig. 3.9 shows the corresponding $\delta$ spectrum.

The intrinsic energy resolution for this experiment was $200 \mathrm{KeV}$ (FWHM) corresponding to a momentum resolution $\Delta \delta=0.018 \%$ (FWHM). A calculation of the quantity $\triangle M M$ through the relationship:

$$
\Delta M M=\frac{\partial M M}{\partial p_{\text {out }}} \cdot 0.01 \cdot \Delta \delta \cdot p_{\text {central }}
$$

with $\partial M M / \partial p_{\text {out }}=0.827 \mathrm{MeV} /(\mathrm{Mev} / \mathrm{c})$ and $\mathrm{p}_{\text {central }}=1382.46$ gives $\triangle M M=207$ $\mathrm{KeV}$, consistent with the result obtained from the fit to the missing mass spectrum. 

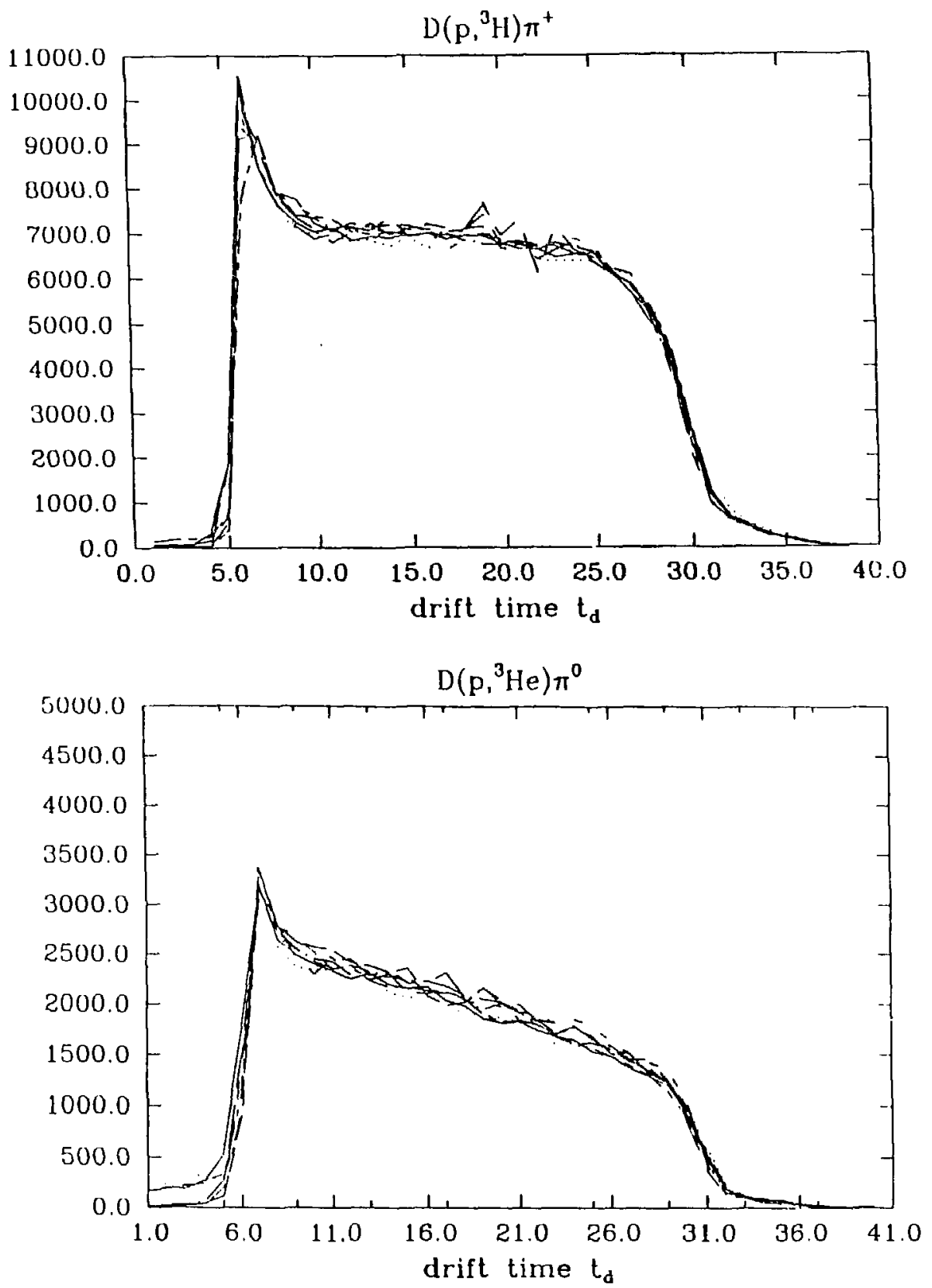

Figure 3.3: Drift time spectra for the detection of ${ }^{3} \mathrm{H}$ and ${ }^{3} \mathrm{Ile}$. 

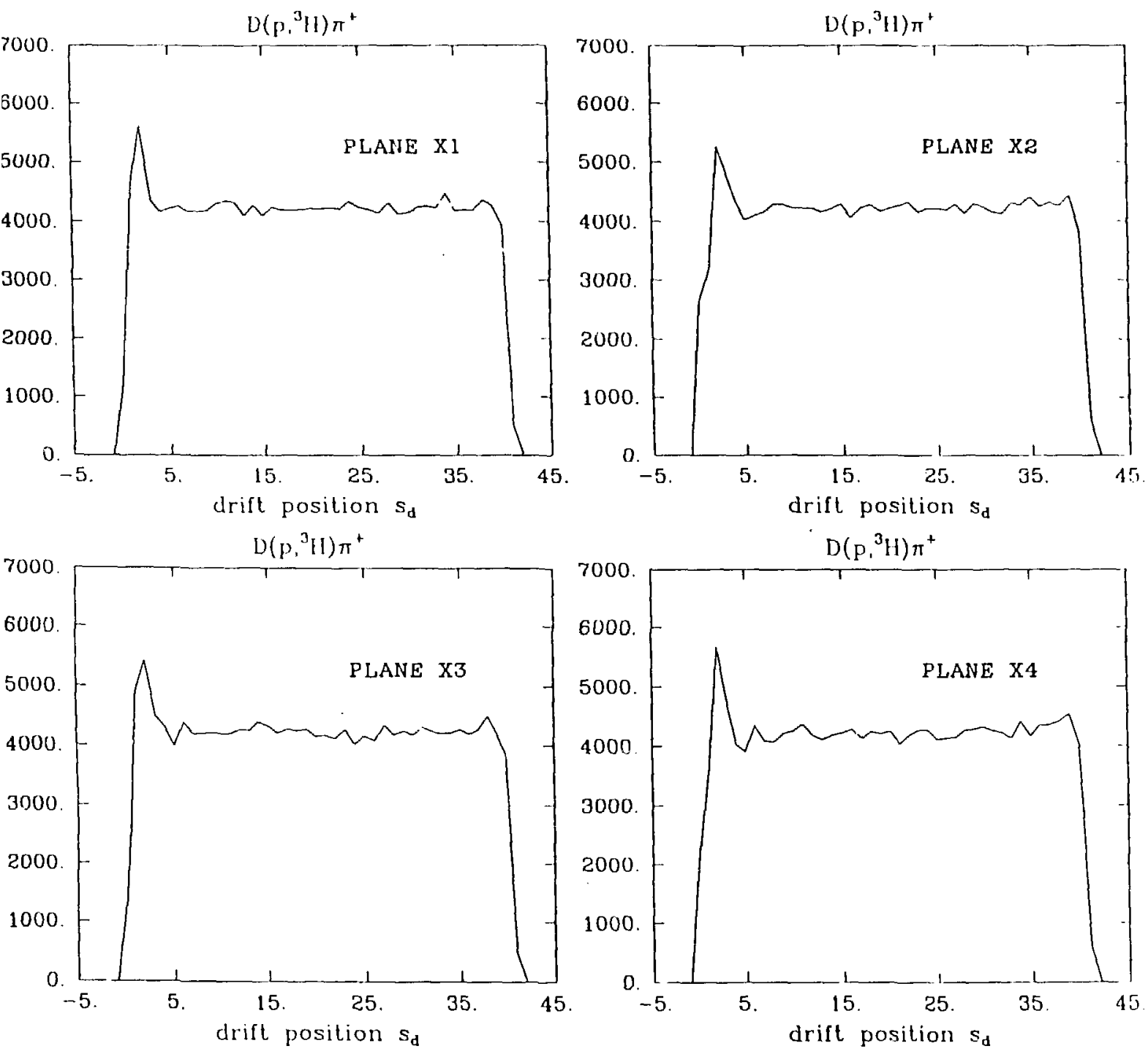

Figure 3.4: Drift distance spectra for ${ }^{3} \mathrm{H}$ (X chambers). 

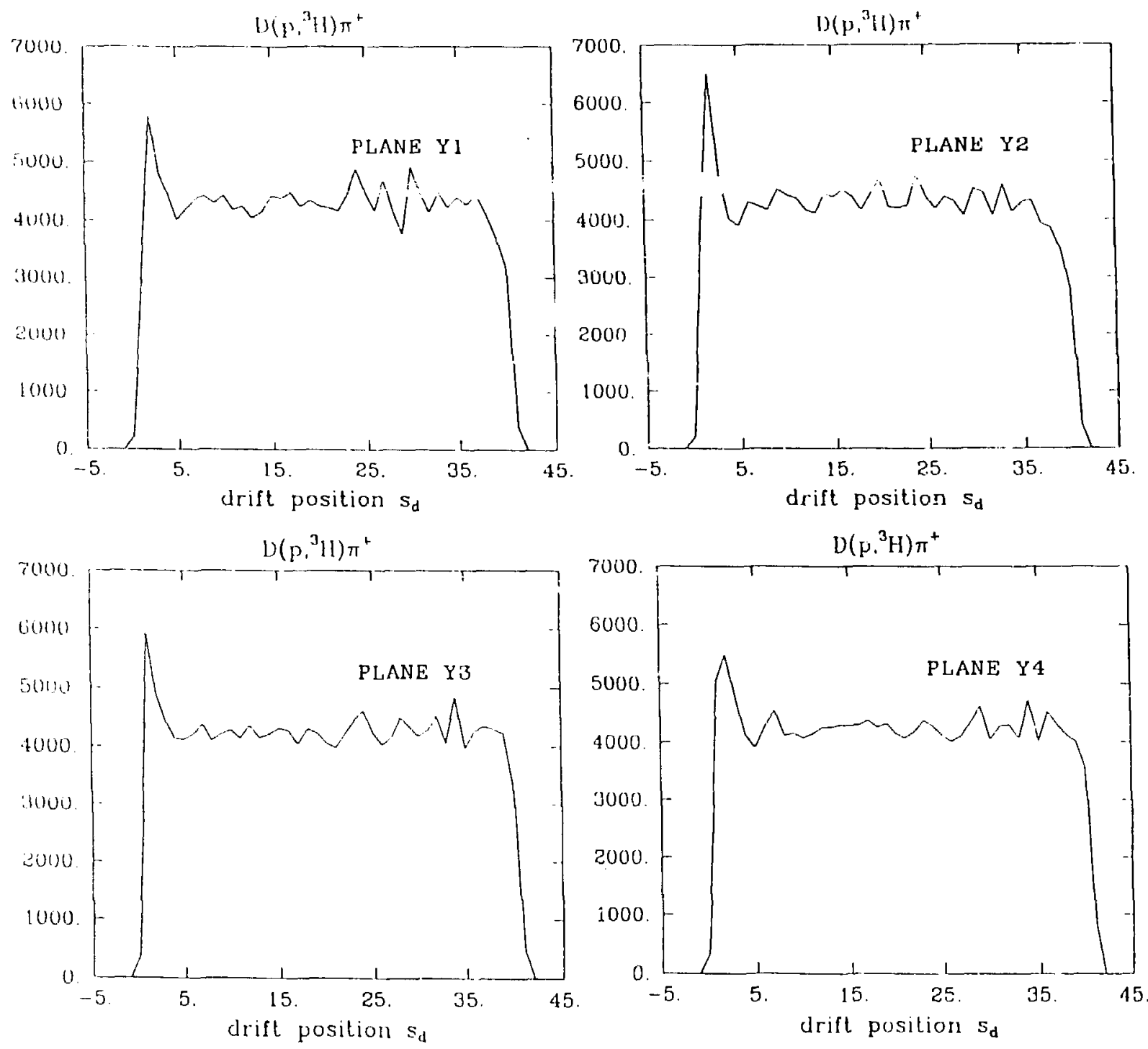

Figure 3.5: Drift distance spectra for ${ }^{3} \mathrm{H}$ (Y chambers). 

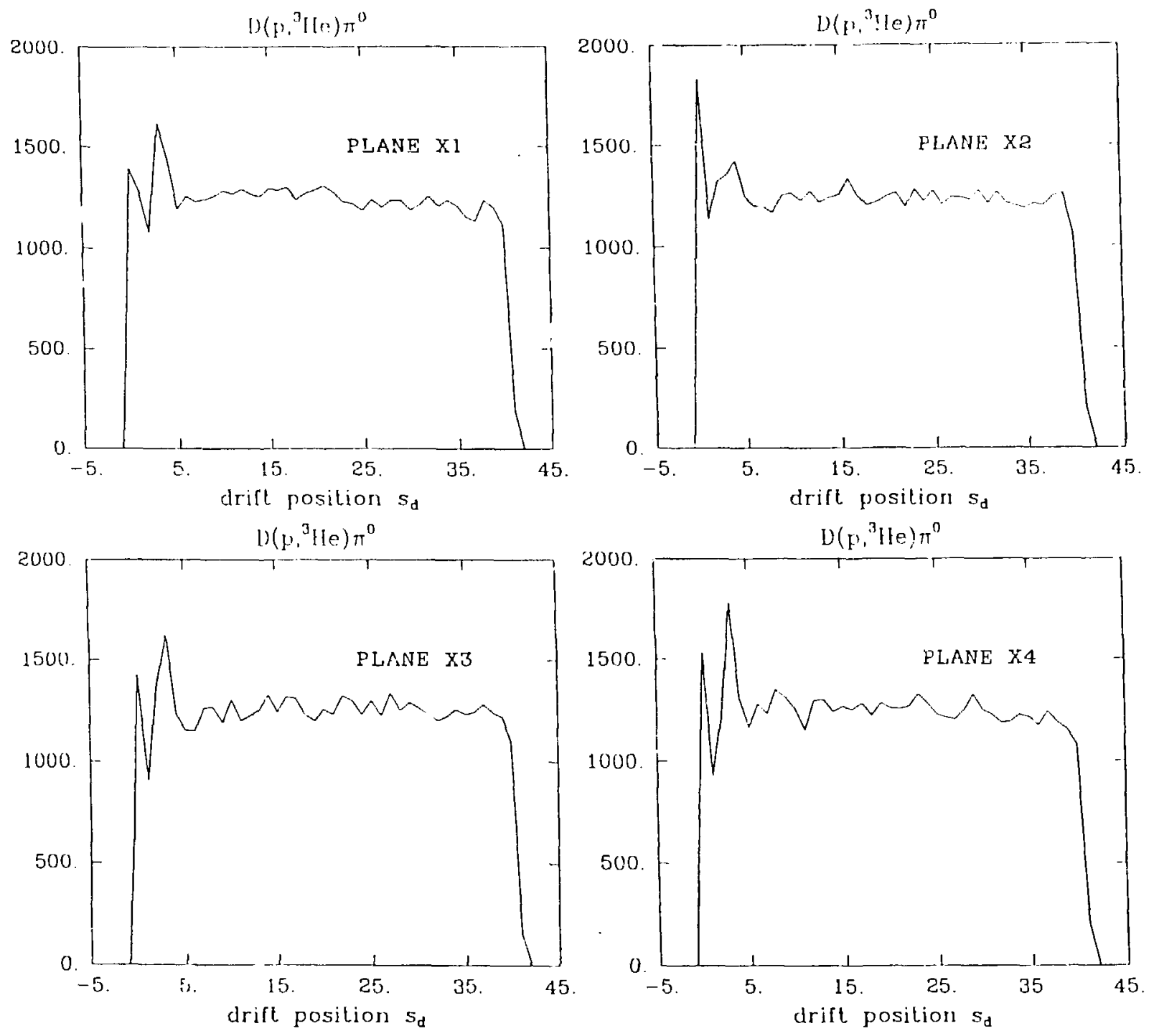

Figure 3.6: Drift distance spectra for ${ }^{3} \mathrm{He}$ (X chambers). 

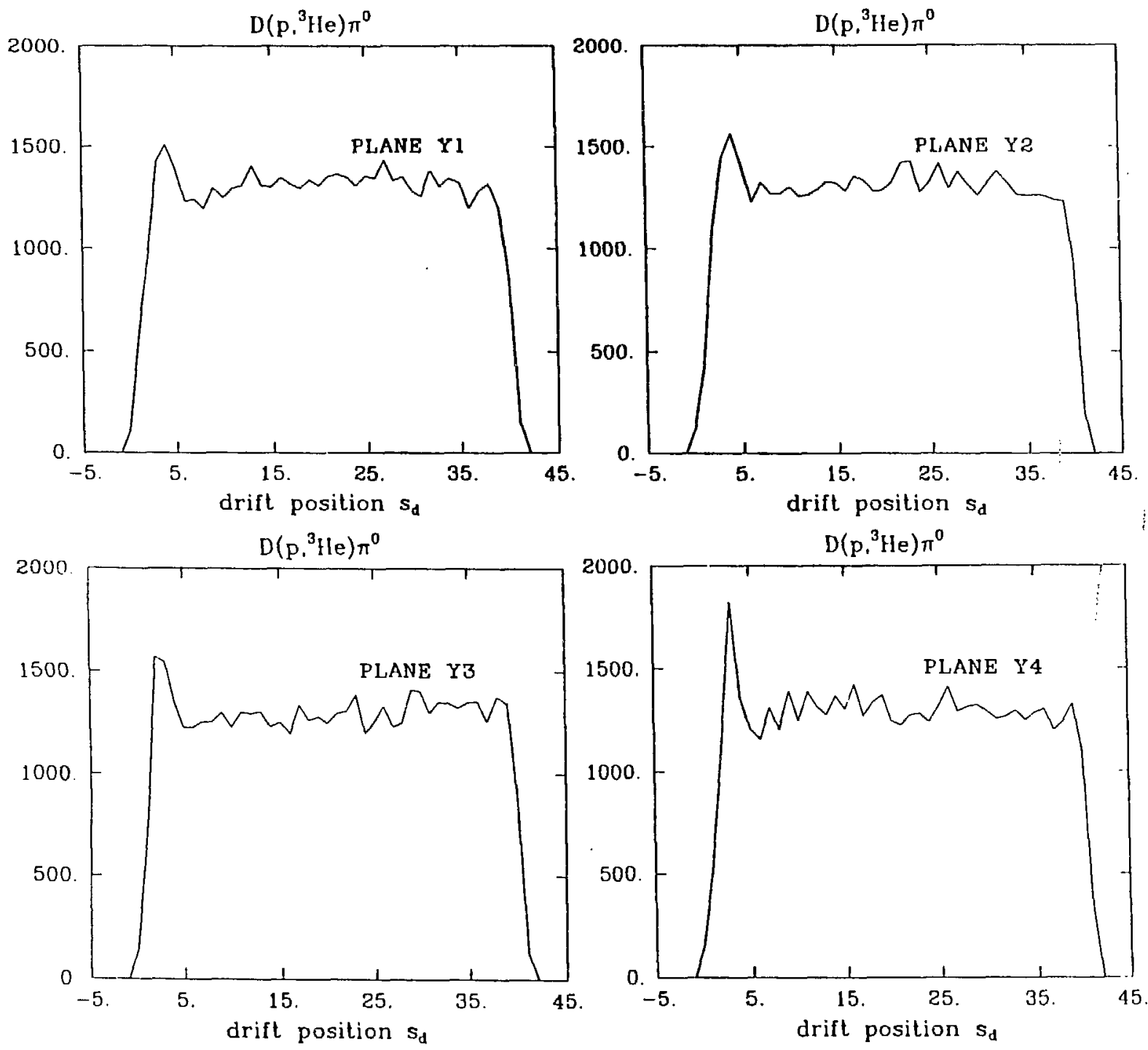

Figure 3.7: Drift distance spectra for ${ }^{3} \mathrm{He}$ (Y chambers). 


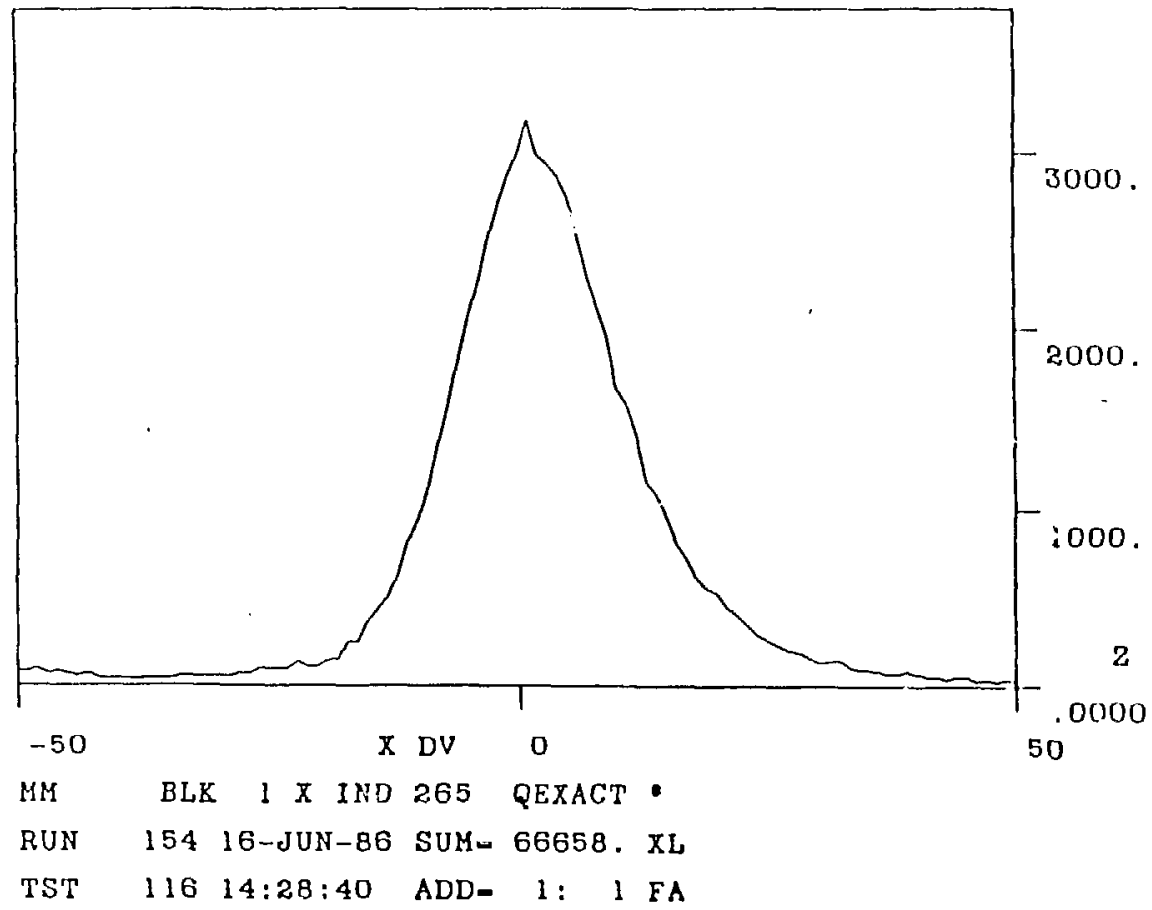

Figure 3.8: $\Lambda$ typical missing mass spectrum for the reaction ${ }^{206} \mathrm{~Pb}(\mathrm{p}, \mathrm{p})^{208} \mathrm{~Pb}$.

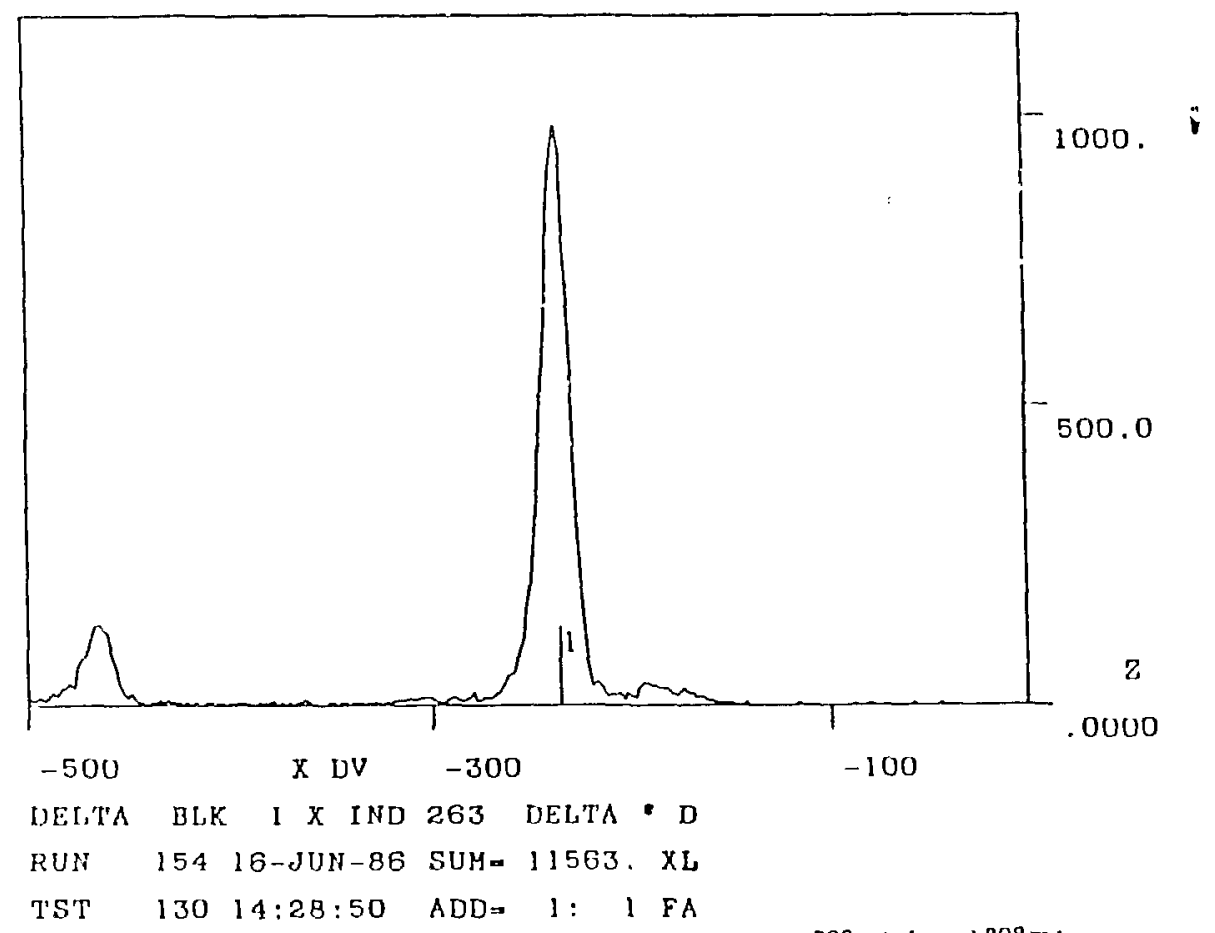

Figure 3.9: $\Lambda$ typical $\delta$ spectrum for the reaction ${ }^{208} \mathrm{~Pb}(\mathrm{p}, \mathrm{p}){ }^{208} \mathrm{~Pb}$ 


\subsection{The good event test.}

In the test file there is a macrotest which defines the 'good event', which selects events which are likely to correspond to the detection of a particle coming from the reaction of interest. The combination of micretests adopted to identify the 'good events' in the analysis of this experiment was somewhat different from the usual definition adopted in processing the data taken at the HRS spectrometer facility. This choice was a consequence of the anomalous performance exhibited by the drift chambers during our experiment.

Two set of tests are imposed on the chamber quantities. A test is imposed on the drift time data words to reject overflows and underflows for these variables. The correct wire identification is verified by examining the variables $\Delta x_{i j}^{m i n}$ defined in the preceeding section. If the value of this variable is greater than a wire spacing, thcre is a corresponding error in wire identification at least in one of the chambers considered. Therefore the test of corrected wire identification is a gate on the variables $\Delta x_{i j}^{m i n}$ with a width of approximately one wire spacing. This test was the one which was critical in our experiment. As Fig. 3.10 shows, in the plane associated with the dispersion direction, the variable $\Delta x_{i j}^{m i n}$ has a distribution characterized by the presence of two additional sizeabie peaks (up to $7.5 \%$ and $\approx$ $4 \%$ of the total event number $i$ espectively) centered at $\Delta x_{i j}^{m i n}=$ w and $\Delta x_{i j}^{\min }=2 \mathrm{w}$, $\mathrm{w}$ being the spacing between signal wires. This effect is present both in the $\mathrm{X}$ and in the $\mathrm{Y}$ chambers. However, this is critical for our experiment only as far as the $\mathrm{X}$ chambers are concerned because of the different momentum spread of the two particles detected.

This effect occurs whenever the tail of the distribution of times associated with one wire exceeds the time shift $\Delta t$ introduced by the delay line between signals coming from adjacent wires. The fraction of events rejected because of this test was anomalously large (up to $30 \%$ ) during our experiment. Table 3.1 shows the summary of the fraction of events which passed this test in three different 

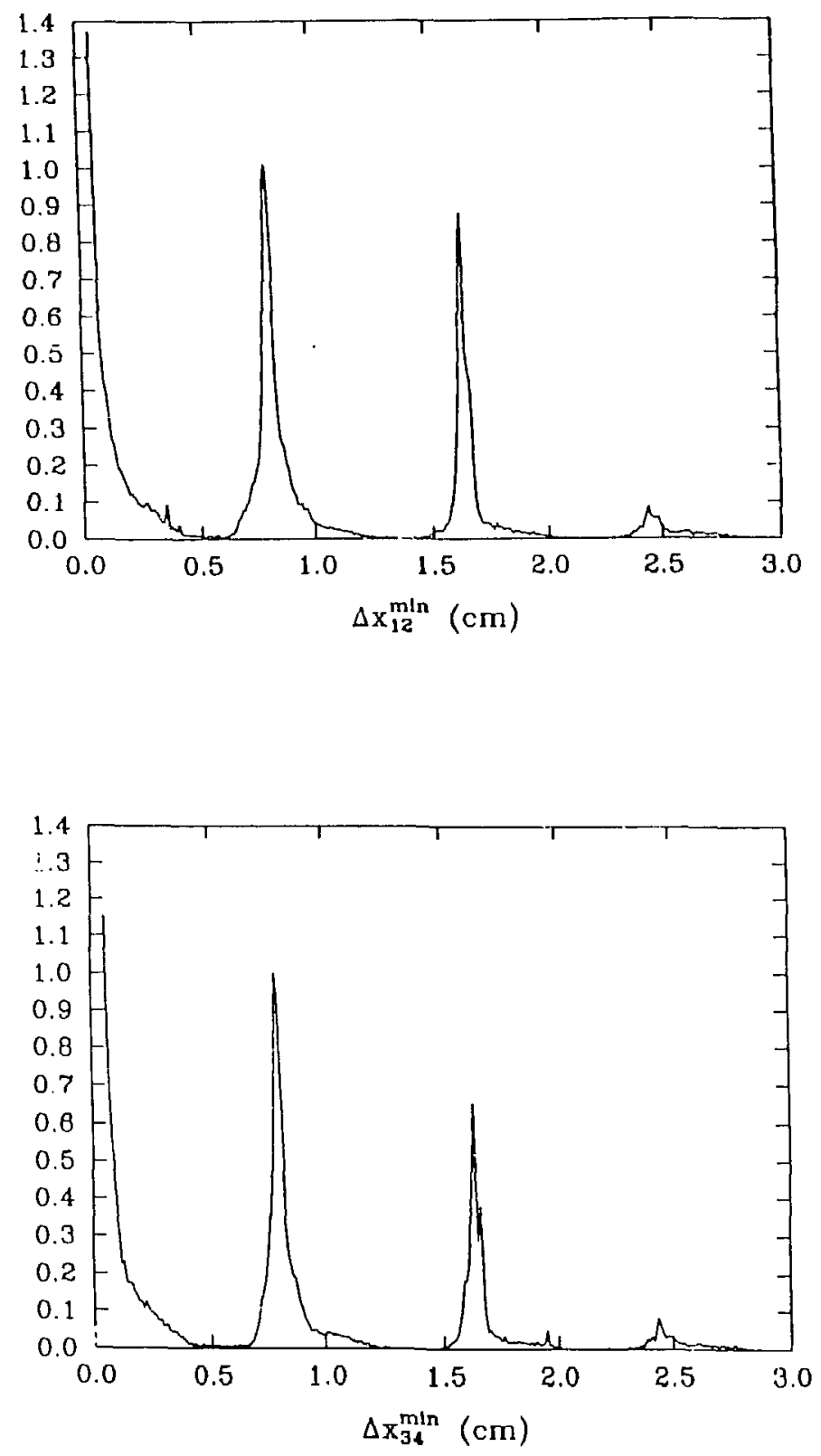

Figure 3.10: Spectra for the wire identification variables in the dispersion direction. 
Table 3.1: Fraction of events passing the wire identification test for the drift chambers used in our experiment.

\begin{tabular}{|c|c|c|c|}
\hline & ${ }^{3} \bar{H}$ detection & ${ }^{3}$ He detection & Continuum proton \\
\hline$\Delta x_{12}$ & $85 \%$ & $69 \%$ & $89 \%$ \\
$\Delta x_{34}$ & $89 \%$ & $73 \%$ & $92 \%$ \\
$\Delta y_{12}$ & $87 \%$ & $78 \%$ & $91 \%$ \\
$\Delta y_{34}$ & $95 \%$ & $84 \%$ & $97 \%$ \\
\hline
\end{tabular}

experimental conditions (detection of ${ }^{9} \mathrm{H},{ }^{3} \mathrm{He}$, continuum calibration run.)

The numbers given represent an average over the active area of the detectors considered. Their difference reflects a difference in overall efficiency of the drift chambers for the detection of the three kinds of particles considered. A difference between ${ }^{3} \mathrm{H}$ and ${ }^{3} \mathrm{He}$ is easy to explain, as the chambers were operated a different voltages in the two cases in order to compensate for the difference in ionizing properties of the two particles. Its should be corrected by the 'chamber efficiency factor', which will be discussed in a subsequent section.

A careful investigation on the chamber performances showed that not only the differential efficiency distribution was different across the different chamber planes, but it also had a shape dependent upon the kind of particle detected. This effect is illustrated in Figs. 3.11 and 3.12 which cumpare the relevant spectra for the two detection conditions.

It can be seen that, particularly in the case of ${ }^{3} \mathrm{H}$, there is a difference in behaviour exhibited by the front and rear chambers. The front chamber planes are characterized by spectra showing sharp peaks, corresponding to local strong oscillations in differential efficiency. The rear chambers have a smoother behaviour, but exhibit nevertheless a rather sharp discontinuity in the neighborhood of $x=0$. The corresponding ${ }^{3} \mathrm{He}$ spectra show some weak similarity to the ${ }^{3} \mathrm{H}$ ones, but have an overail greater uniformity.

The final analysis involves the integration of the data over some angular range, and, consequently, over some $x_{f}$ interval corresponding to the range of 

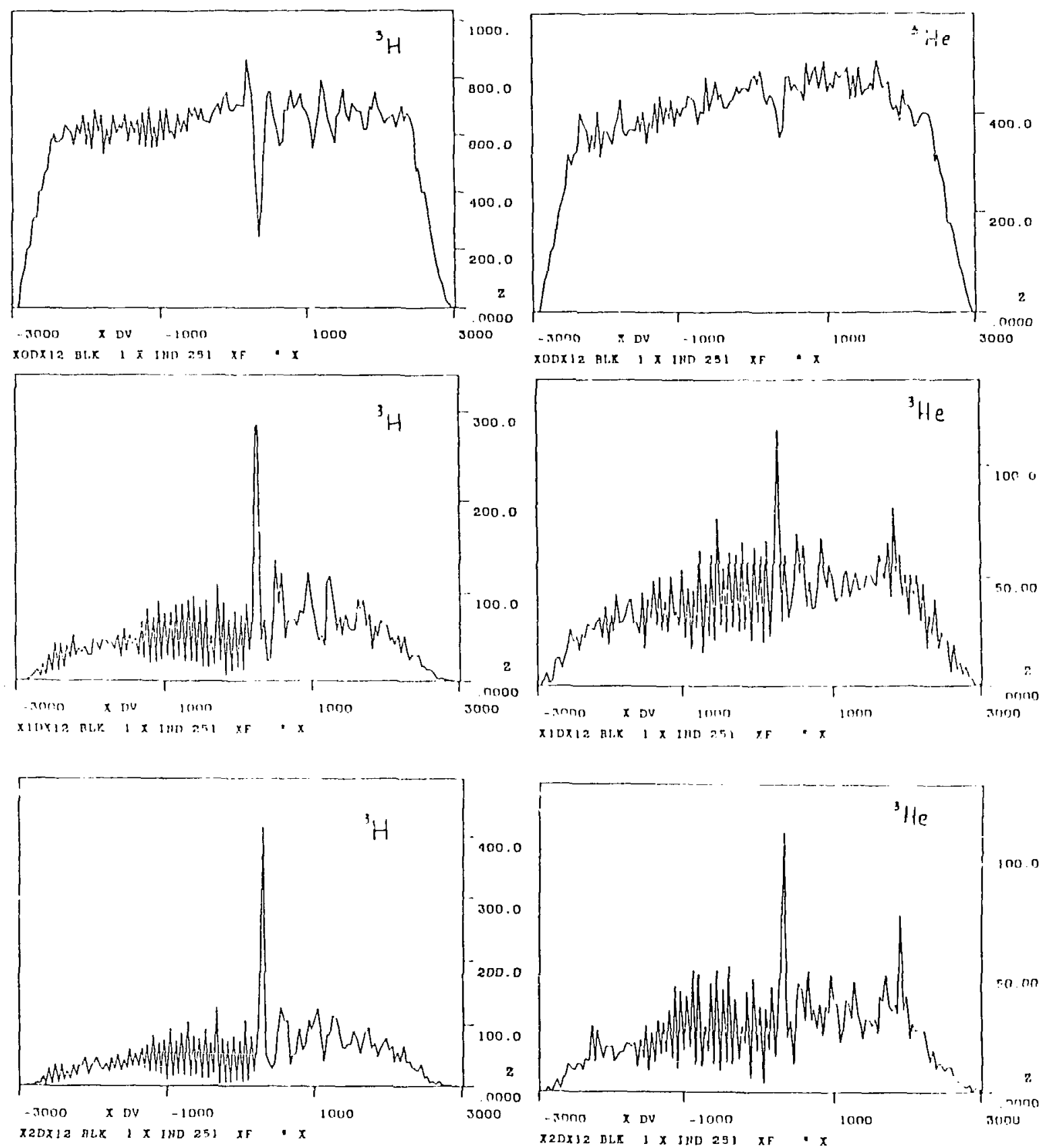

Figure 3.11: $X_{f}$ distribution of the events corresponding to 0 (Lop), 1 (middle), and 2 (bottom) wire difference in extrapolated position for the front chambers $\mathrm{C}_{1}, \mathrm{C}_{2}$. 

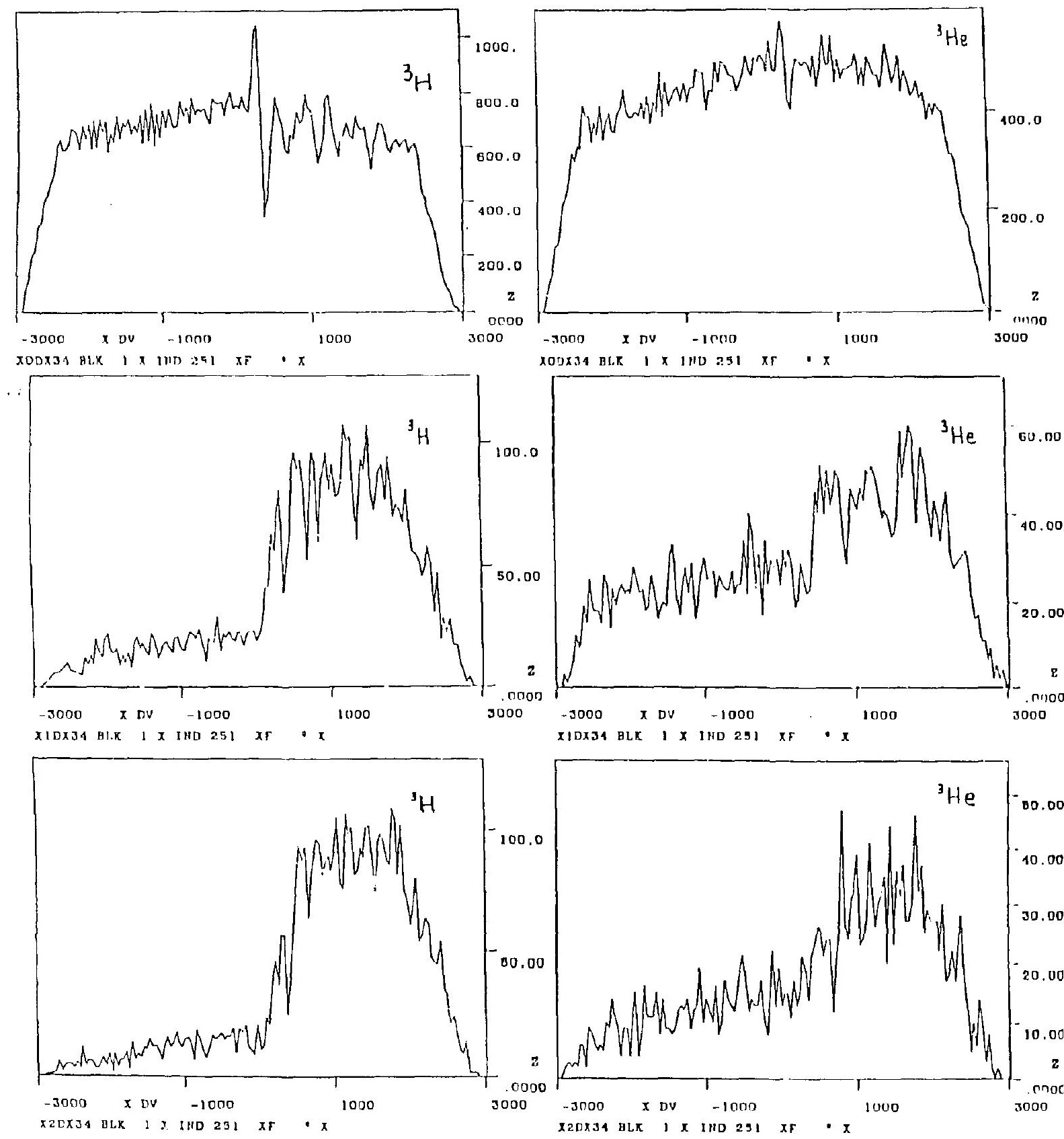

Figure 3.12: $X_{f}$ distribution of the events corresponding to 0 (top), 1 (middle), 2 (bottom) wire diffcrence in extrapolated position for the rear chambers $\mathrm{C}_{3}, \mathrm{C}_{4}$. 


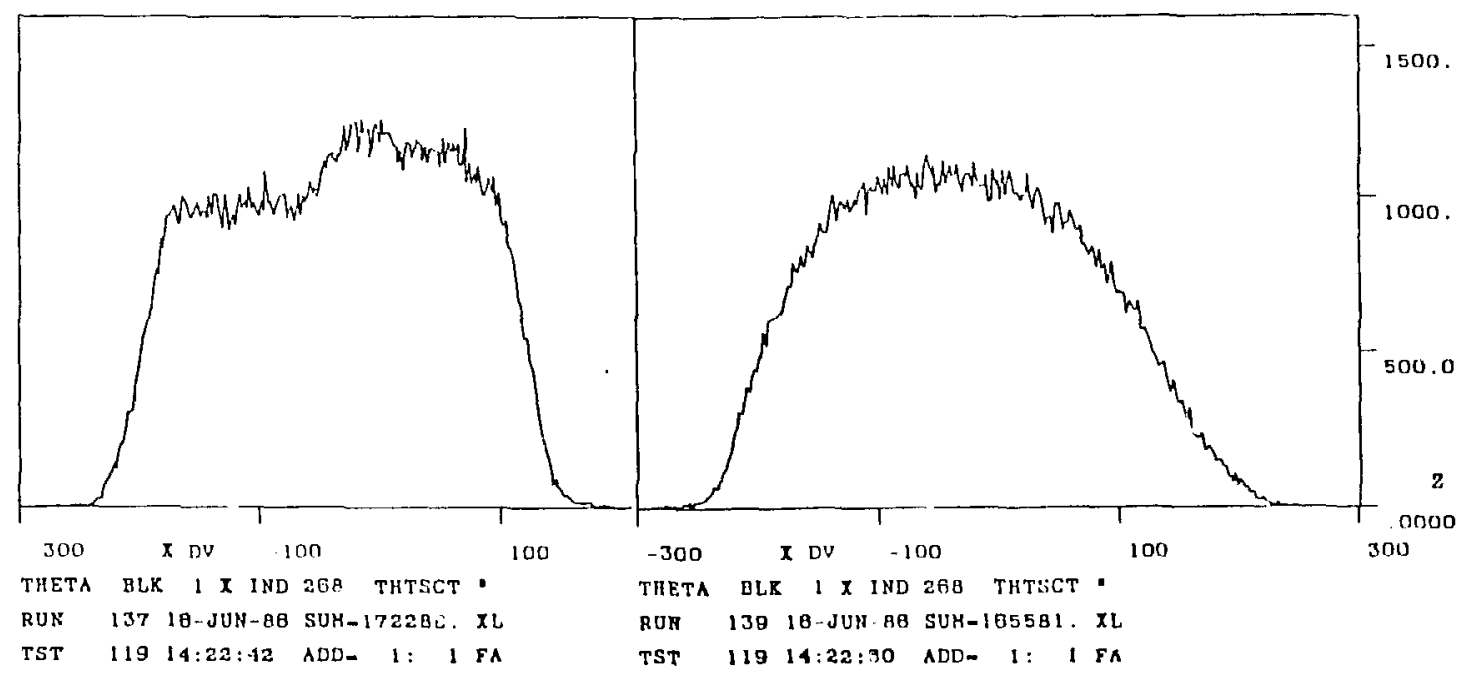

Figure 3.13: Scattering angle spectra gated by the conventional good event test.

momenta spanned by the detected particles scattered in this angular rcgion. Unfortunately the $x_{f}$ regions corresponding to the same angular interval are not the same for ${ }^{3}$ II and ${ }^{3}$ Ile because of their different distribution of energy loss in the target. This effect is illustrated by Fig. 3.13, which shows the angular spectra for ${ }^{3} \mathrm{II}$ and ${ }^{3}$ Ile gated by the conventional good event (corresponding to the selection of the events beionging to the first peak of $\Delta x_{i j}^{\text {min }}$.

The effect on the ${ }^{3}$ II spectrum produced by the differential efliciency distri. bution is evident. $\Lambda$ s small scattering angles are associated with higher average momenta of the detected particle, there is a discontinuity in the spectrum indicating that in this region there is on an average a lower efliciency.

There is one consideration which makes this effect less critical. As the interest of the experiment is concentrated in the acrurate determination of integral quantities which do not have a sharp energy dependence, the monentum resolution of the reconstructed event is not a relevant parameter. The acceptance of events corresponding to $\Delta x_{i j}^{\min }$ equal to two drift cells corresponds to a fractional 


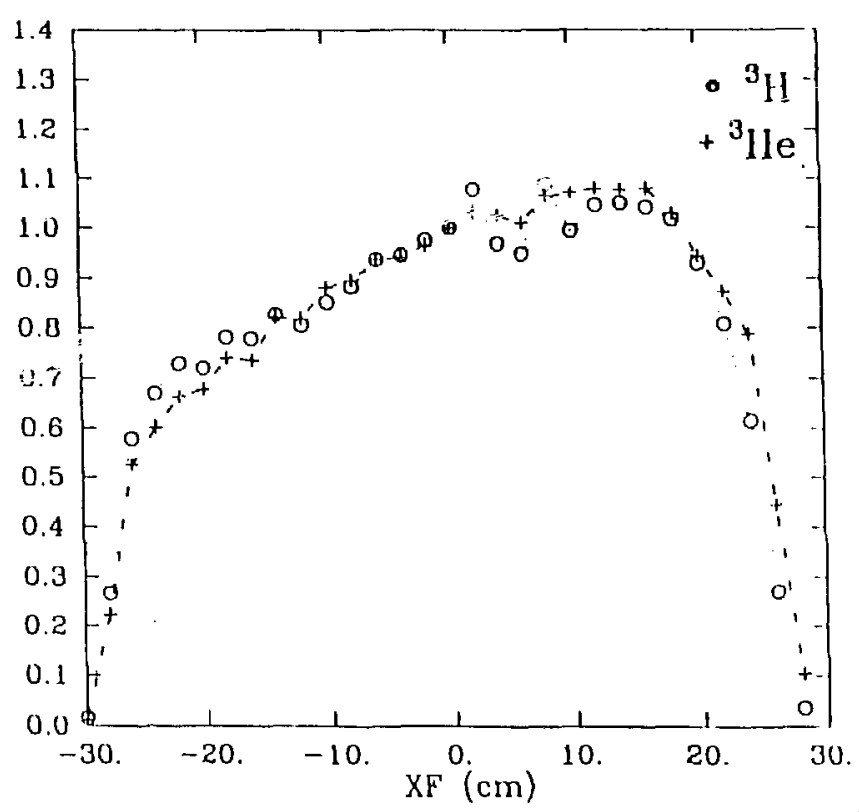

Figure 3.14: Normalized $x_{f}$ distributions for the reactions $\mathrm{D}\left(\mathrm{p},{ }^{3} \mathrm{H}\right) \pi^{+}$and $\mathrm{D}\left(\mathrm{p},{ }^{3} \mathrm{IIe}\right) \pi^{0}$ gated by the 'extended good event' test.

momentum uncertainty $\Delta \delta$ equal to $0.087 \%\left(\Delta p_{\text {out }} \approx 1.3 \mathrm{MeV} / \mathrm{c}\right)$ which is irrelevant with respect to the other sources of missing mass broadening discussed in the section describing the Monte Carlo simulation performed. This suggests that we can adopt an 'extended good event' test, accepting $\Delta x_{i j}$ in the range between 0 and two drift cells without any harm to our interests. Fig. 3.14 shows a comparison between the normalized $x_{f}$ spectra for ${ }^{3} \mathrm{H}$ and ${ }^{3} \mathrm{He}$. It can be seen that the average agreement between the two spatial distributions is very satisfactory.

Further support for this choice is provided by the comparison between the angular spectra corresponding to the two reactions. As seen if Fig. 3.15 the discontinuity present in the case of ${ }^{3} \mathrm{H}$ shown in Fig. 3.13 is removed by adopting this test.

The other conditions applied in the selection of 'good events' are conventional. They involve the particle identification test discussed in the previous chapter and the so-called 'xang cut'. The latter has the purpose of rejecting the particles following peripheral trajectories within the spectromelcr, which are likely to have their trajectory altered by scattering from the gap walls. The scatter 


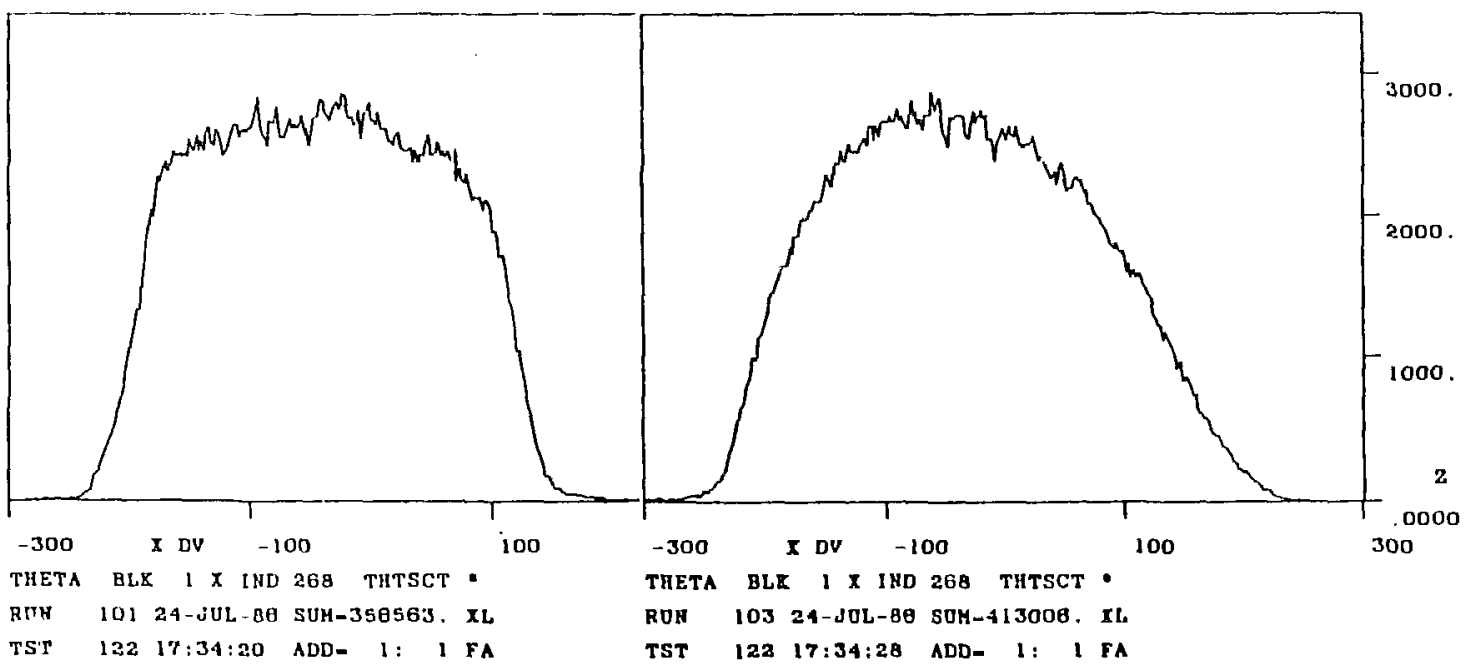

Figure 3.15: Scattering angle spectra gated by the 'extended good event' test. Compare with Fig. 3.13.

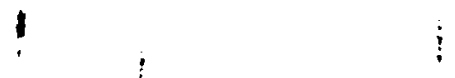

plots corresponding to these two tests are given in Figs. 3.16 and 3.17 respectively. . 


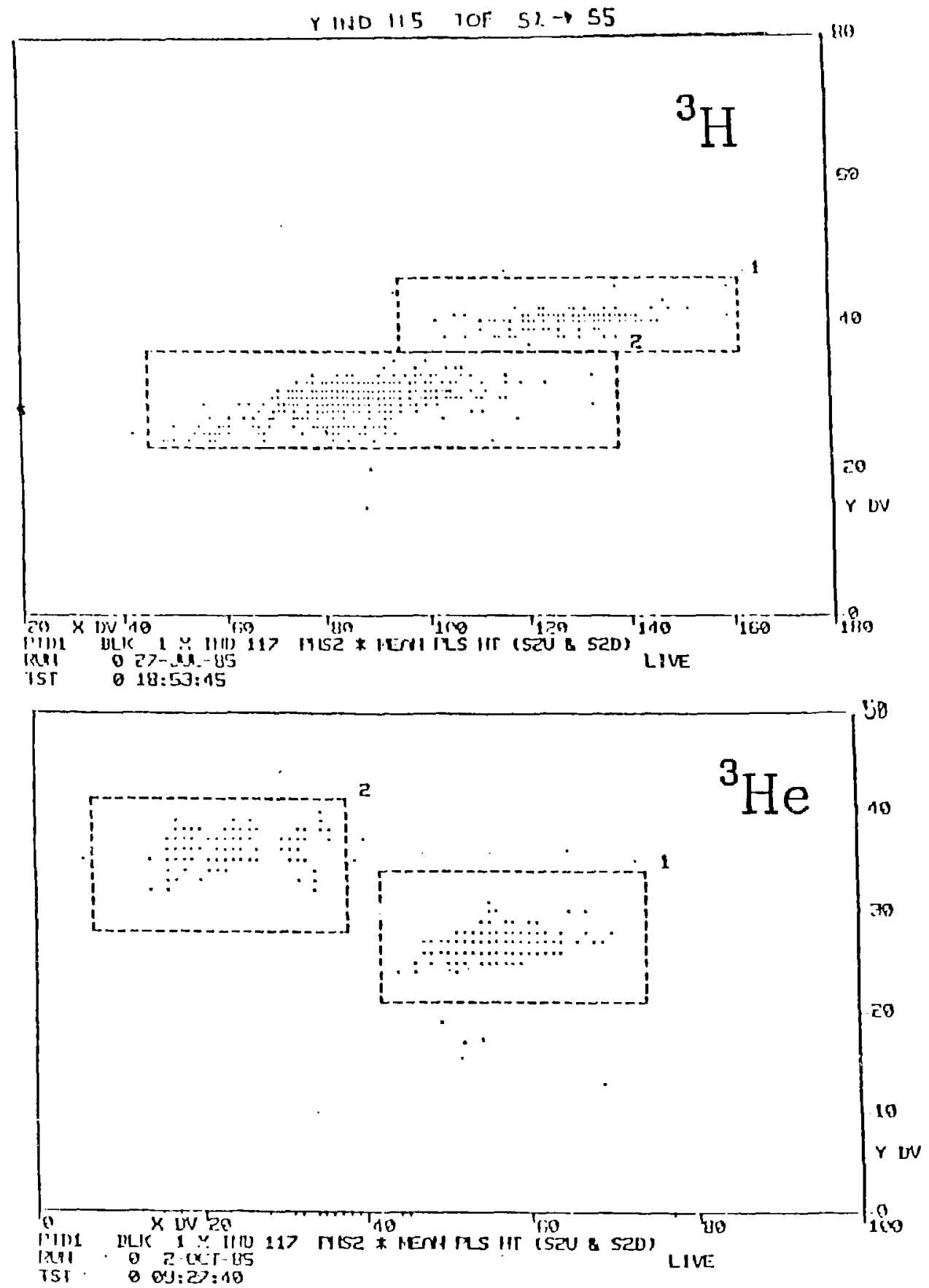

Figure 3.16: Dot plots for ${ }^{3} \mathrm{H}$ and ${ }^{3} \mathrm{He}$ identification, abscissa are pulse heights and ordinates are time of flight. (box 1 contains the particles of interest in both cases.) 


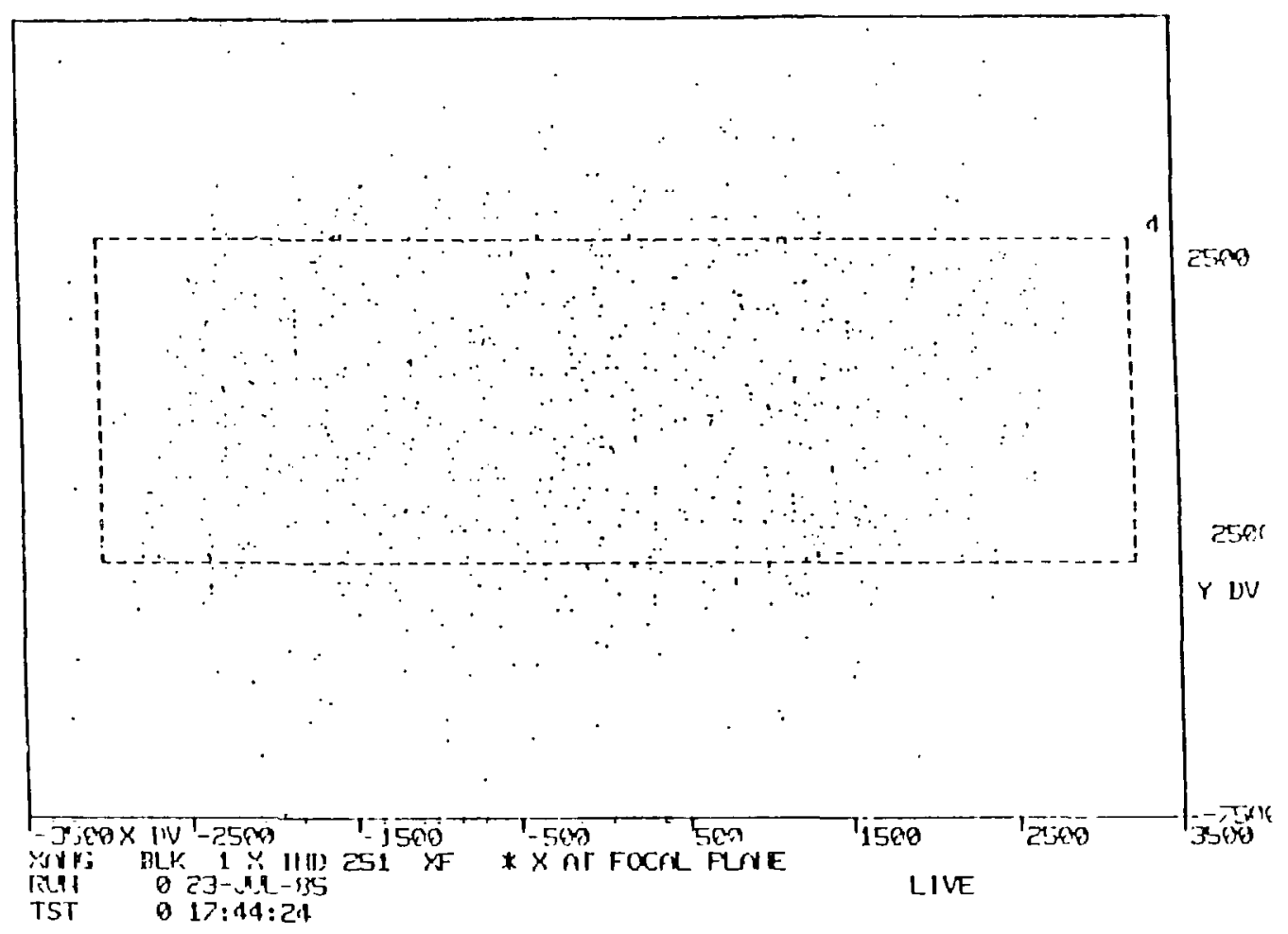

Figure 3.17: Dot plot for XANG cut. The abscissa is $x_{f}$ and the ordinate is related to the angle $\theta_{t}$. Box 4 defines the fiducial space. 


\subsection{The method of analysis.}

A choice is available in order to obtain the yields from which the observables of interest are calculated. It is possible to integrate the angular spectra in selected intervals or to analyze the missing mass peaks gated by a specific angular interval.

The latter solution was adopted in the analysis of this experiment. The motivation for this choice is illustrated in Fig. 3.18 which shows the tails of the missing mass peaks for normal and reverse polarization superposed and normalized with respect to the beam monitor counts accumulated during the respective polarization states and the peaks normalized with the same method. It can be seen that in the tail regions displayed, the ratio normal/reverse $\approx 1$, which implies an analyzing power very close to zero $\left(\left|A_{v}\right| \leq 0.1\right)$. In the region of the main peaks $A_{\mathrm{y} 0} \approx 0.4$ which gives normal/reverse $\approx 2$. Near-zero analyzing power indicates the dominance of background events in the tail regions. These events are most likely produced in the material (mostly mylar and alumnized mylar) surrounding the active volume of the target. One needs to subtract these background events in order to obtain the true results. This is possible only with the missing miss spectra, as the background produces no structure in the scattering angle spectrum.

In order to evaluate the correctness of our background subtraction procedure the distribution of the analyzing power as a function of the missing mass examined by calculating $A_{\nu 0}(M M)$ for the two reactions of interest, with intervals of integration $6 \mathrm{MeV}$ wide. The results for ${ }^{3} \mathrm{H}$ and ${ }^{3} \mathrm{He}$ respectively are shown in Fig. 3.19 where the shapes of the MM spectra have been also superposed.. It can be seen that these distributions allow an identification of the region where 'signal' events are dominant and the region where 'background' events are do:ninant. Because of the wider range of energy loss at the target, the ${ }^{3} \mathrm{He}$ 'signal' peak has a greater spread in missing mass. This feature makes it more difficult to obtain a reliable separation of the background and it also induces a greater sensitivity of the final results to the subtraction performed. One consideration which makes 

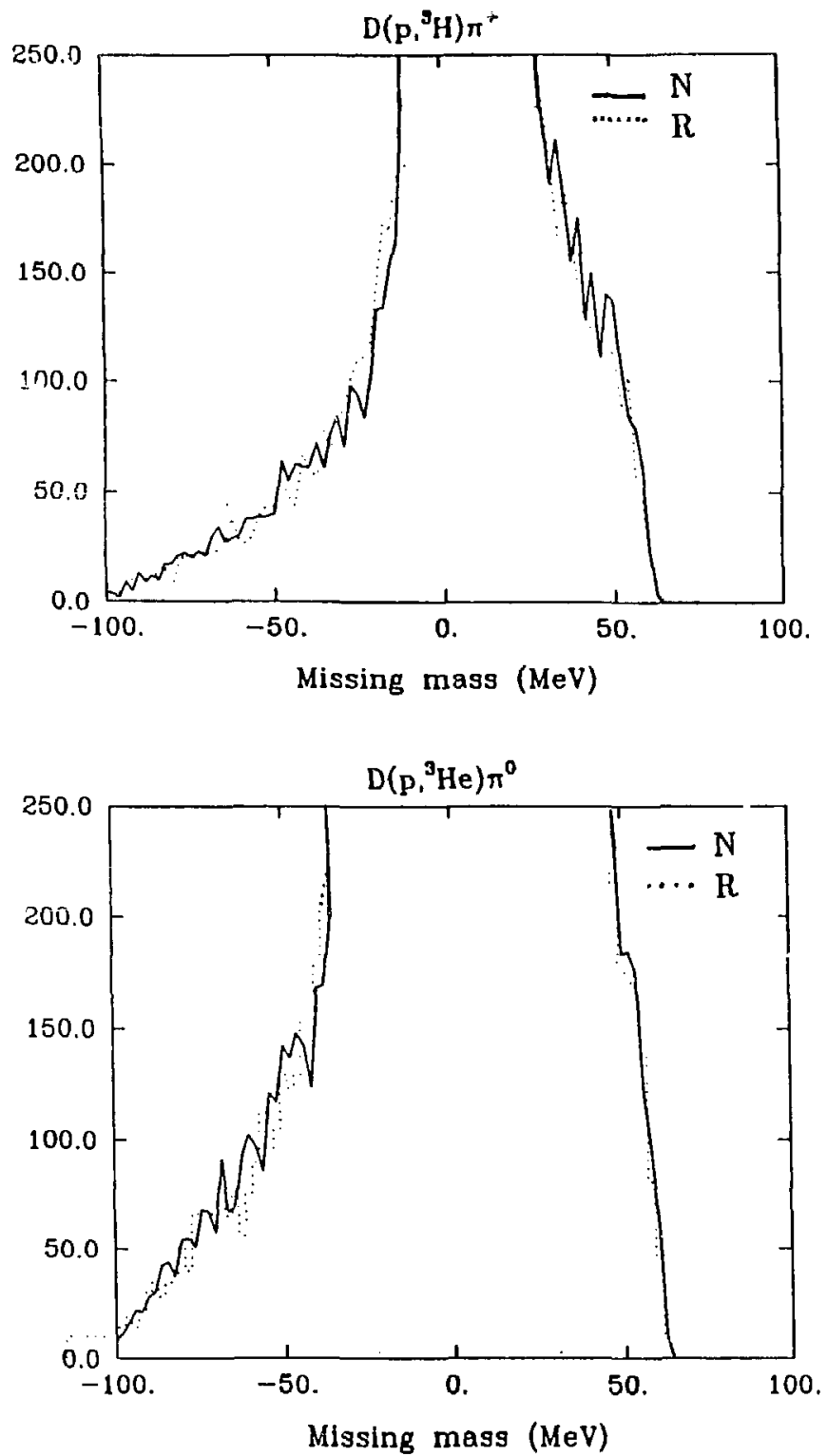

Figure 3.18: Comparison of the yields in the tail region the missing mass spectra for normal and reverse polarization 

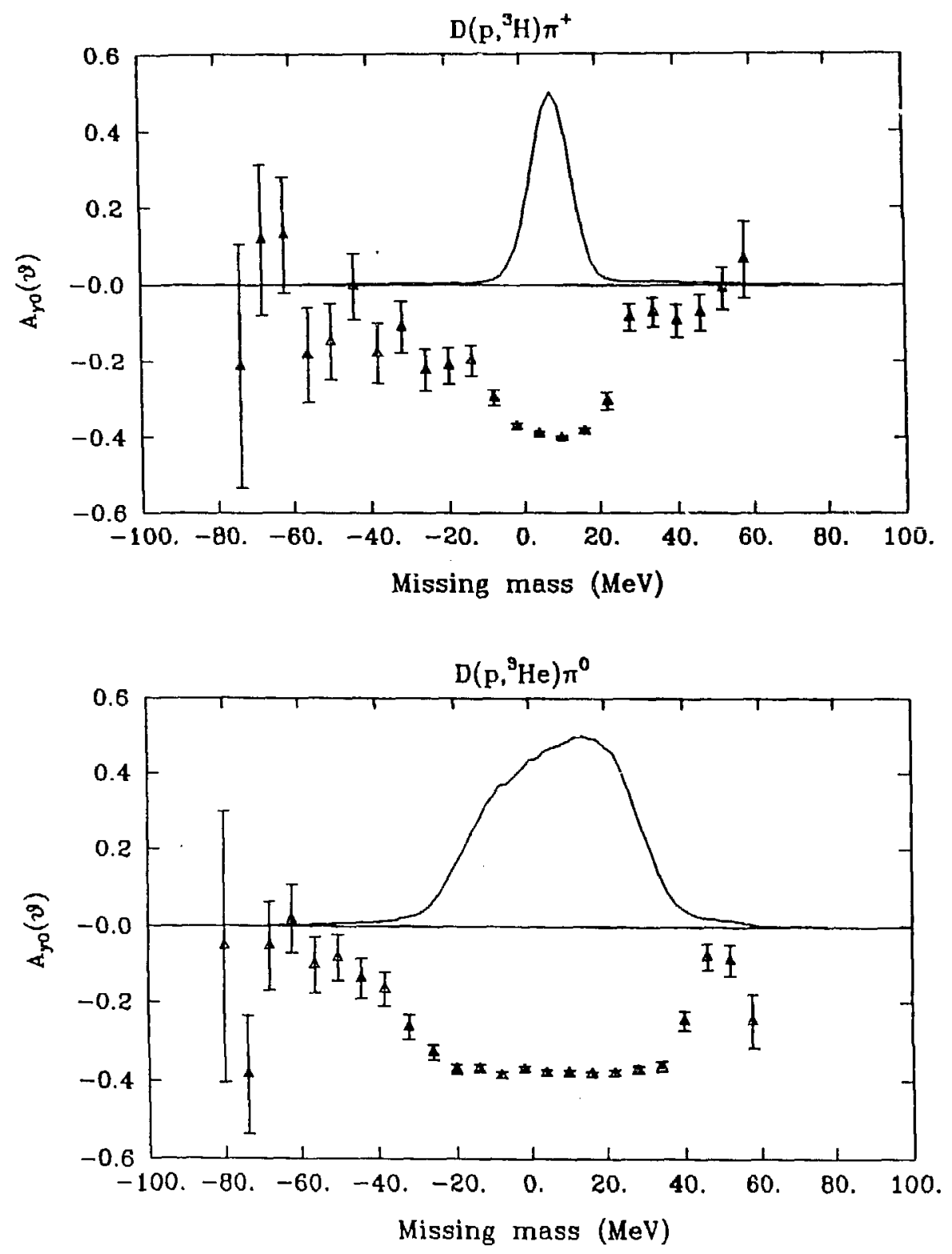

Figure 3.19: $A_{y 0}$ as a function of missing mass for the two reactions (no background subtraction has been done). The shape of the missing mass peak is shown in each case to illustrate the weight of different regions of missing mass. 
this problem less severe is the similarity between the shape of the tails of the missing mass spectra for ${ }^{3} \mathrm{II}$ and ${ }^{3} \mathrm{He}$. This property is illustrated in Fig. 3.20, which shows th' missing mass spectrum for ${ }^{3} \mathrm{II}$ scaled by a factor $M$ with respect to the corresponding one for ${ }^{3} \mathrm{He}$, where $M$ is given by:

$$
M=\left(\frac{E R O 4}{C F}\right)_{3_{H e}} /\left(\frac{E R O 4}{C F}\right)_{{ }_{3}}
$$

where:

- ERO4 refers to the counts of the beam integrator and

- CF is the correction factor for chamber efficiency and computer livetime.

This comparison led us to the conclusion that the most reliable way to proceed was to extract the background distribution from ${ }^{3} \mathrm{H}$, where the 'signal' peak is less wide and to subtract a distribution with the same shape scaled by a factor $M$ from the corresponding ${ }^{3}$ He specirum. This choice is supported also by the Monte Carlo simulation, which was performed for ${ }^{3} \mathrm{II}$ and ${ }^{3}$ IIe background events separately and showed no difference between the distribution in missing mass for the two cases. The analysis of the tails of the ${ }^{3} \mathrm{H}$ spectrum allowed an estimate of the analyzing power of the background to be $-0.03 \pm 0.06$. The shape of inissing mass spectrum associated with this kind of event was obtained from the Monte Carlo program. This spectrum was normalized with respect to the experimental one in the tail region on the low missing mass (between -70 . and -40 . MeV). The normalized background spectrum was empirically corrected at the missing mass boundary regions in order to match the experimental distribution. The limited ability of the program in reproducing the $M M$ distribution in these regions can be understood in terms of the fact that the Monte Carlo simulations for $x_{f}$ spectra show significant differences from the experimental distributions at the extreme of the acceptance (sce Figs, 2.11 and 2.12). Fig. 3.21 shows the missing mass spectra for ${ }^{3} \mathrm{Il}$ and ${ }^{3} \mathrm{II}$. The background distribution subtracted is indicated in dotted lines.

Perhaps a $\mathrm{ml}$ : satisfactory way to determine background would have been 


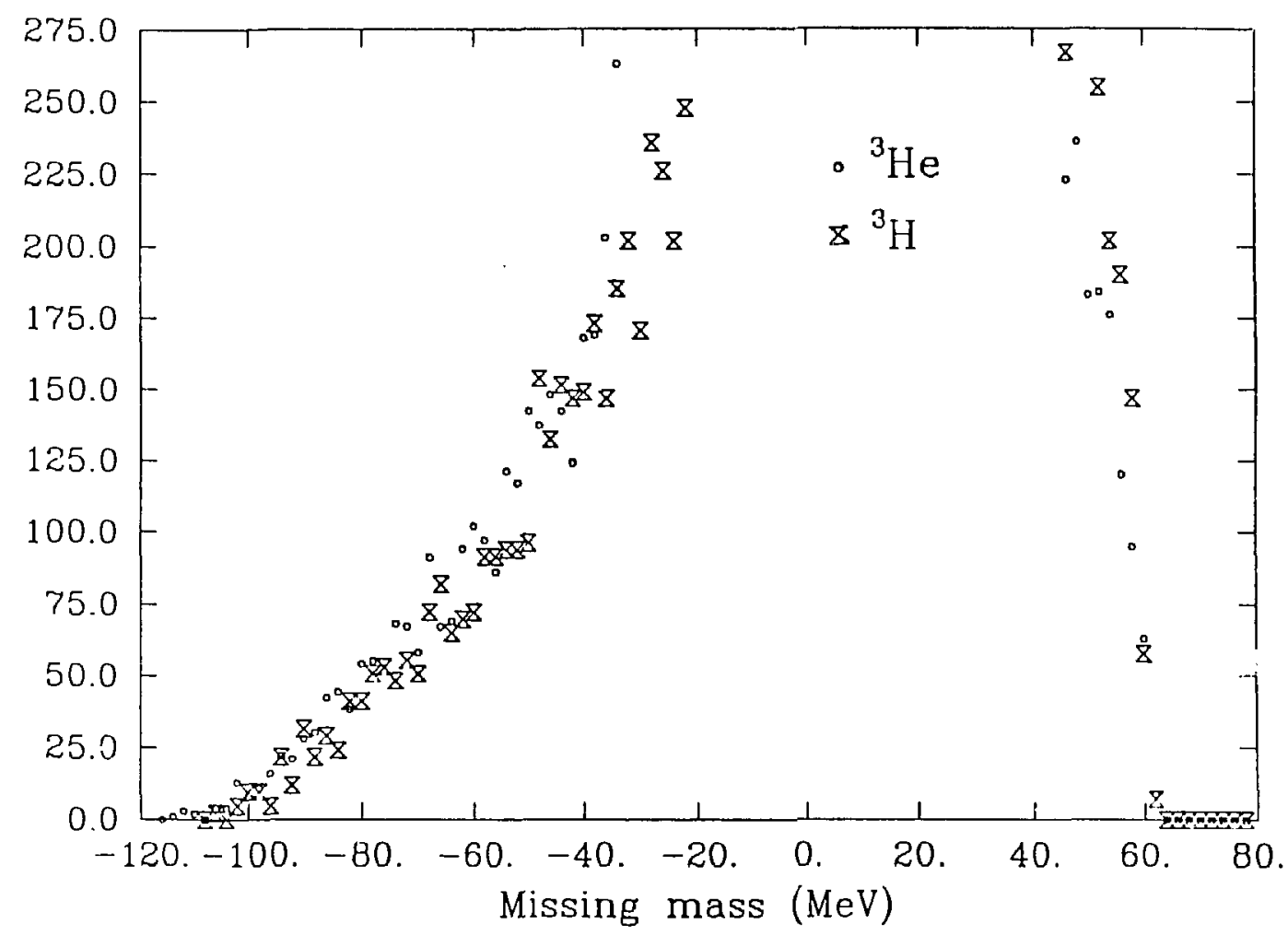

Figure 3.20: Comparison between the tails of the normalized missing mass spectra for ${ }^{3} \mathrm{H}$ and ${ }^{3} \mathrm{He}$ detection. 

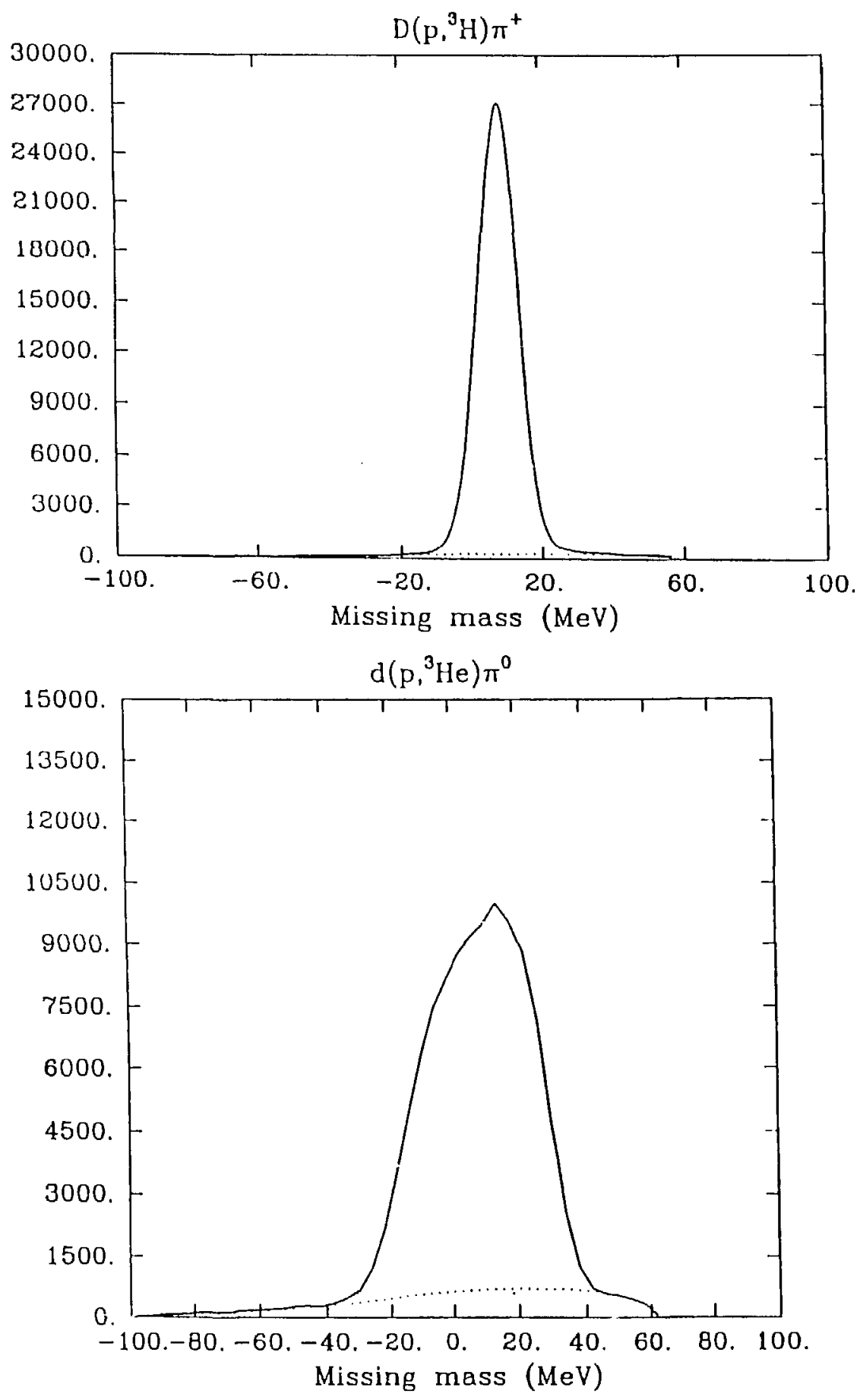

Figure 3.21: Missing mass spectra for the two reactions. The dotted curves indicate the background level. 
to measure it. This would have required us to take data with the liquid target container filled with hydrogen and the magnetic spectrometer at the two field settings corresponding to ${ }^{3} \mathrm{H}$ and ${ }^{3} \mathrm{He}$ detection. The hydrogen filling is required to reproduce the spatial distribution of the target flask (bulging) and to generate the same energy loss distribution as during the runs corresponding to the reactions of interest. During this experiment it was not possible to perform these background runs because of failure of the mechanism for emptying the target and refilling it with hydrogen.

Another choice to be made in analyzing the data was the angular gate to apply to the missing mass spectra. The statistical accuracy is proportional to the width of the interval of angular integration $\Delta \theta_{s c}$. In addition, the region of the drift chambers which is illuminated by the events increases proportionally to $\Delta \theta_{o c}$. The efficiency correction, which will be discussed later is an integral quantity, determined by the average behaviour of the individual chamber planes. It is therefore more appropriate for wider $x_{f}$ intervals, over which the effects of the details of the differential efficiency are averaged out. These considerations suggest the choice of a wider angular gate. On the other hand, the effect of the different distortions of the angular acceptance curve for ${ }^{3} \mathrm{H}$ and ${ }^{3} \mathrm{He}$ due to the difference in their energy loss at the target becomes more serious for wider angular acceptance. The choice of a single angular interval is not easy. We have therefore analyzed the data with two different angular cuts. The efficiency for each angular gate $\epsilon(\Delta \theta)$ was obtained from the Monte Carlo simulation. The difference between the results obtained in the two angular gates should give us a quantitative indication of the uncertainty of the correction applied. As we shall see later the difference was found to be well within the specified errors.

Another remark must be made in connection with the angular integration performed. Equal laboratory angles of the heavy reaclion products correspond to different $\pi$ angles in the center of mass reference frame, because of the difference of mass between $\pi^{r}$ and $\pi^{0}$ of the two reactions. In order to remove this kinematical 
Table 3.2: $\Lambda$ verage angles for the observables measured.

\begin{tabular}{|c|c|c|c|c|c|}
\hline \multicolumn{3}{|c|}{${ }^{3} \mathrm{H}$} & \multicolumn{3}{|c|}{${ }^{3} \mathrm{He}$} \\
\hline$\Delta \theta_{\text {lab }}(\mathrm{deg})$ & $\left(\overline{\theta_{l a b}}\right\rangle$ (deg) & $\left\langle\theta_{c m}^{\pi}\right\rangle(\mathrm{deg})$ & $\Delta \overline{\theta_{l a b}}(\mathrm{mrad})$ & $\left\langle\overline{\theta_{l a b}}\right\rangle(\mathrm{deg})$ & $\left\langle\overline{\theta_{\mathrm{c} m}^{\pi}}\right\rangle$ (deg) \\
\hline $11.21-12.13$ & 11.674 & 130.520 & $11.26-12.17$ & 11.715 & 130.528 \\
\hline $11.09-12.47$ & 11.778 & 130.033 & $11.14-12.52$ & 11.810 & 130.085 \\
\hline
\end{tabular}

effect, the angular regions of integration were chosen with a relative displacement such that the corresponding average $\pi$ angle in the center of mass was the same for the two reactions to bo compared. Table 3.2 gives the ranges employed for ${ }^{3} \mathrm{H}$ and ${ }^{3}$ He respectively and the corresponding average laboratory angle of the detected particle and the $\pi$ angle in the center of mass. 


\subsection{Calculation of $R(\theta)$ and $A_{y 0}(\theta)$.}

The normalized yields used in the calculation of the ratio $R(\theta)$ and $A_{y 0}(\theta)$ were obtained through the relationships:

$$
\begin{aligned}
& Y_{n}^{\prime}=\frac{C F_{n}^{\prime} \cdot Y_{n}}{E R 04_{n} \cdot \epsilon_{n}(\Delta \theta)} \\
& Y_{r}^{\prime}=\frac{C F_{r} \cdot Y_{r}}{E R 04_{r} \cdot \epsilon_{r}(\Delta \theta)}
\end{aligned}
$$

where:

- the subscripts $n$ and $r$ refer to the beam polarization state (normal or reverse), - $Y$ is the yield obtained by integrating the missing mass spectrum in a selected interval after having performed background subtraction,

- $C F$ is the correction factor for chamber efficiency and computer livetime,

- ER04 is the reading of the scalers associated with one of the beam integrators located in the scattering chamber,

$-\epsilon(\Delta \theta)$ is the detection efficiency in the angular gate considered as obtained from the Montecarlo simulation.

The computer livetime $\tau_{c}$ is obtained by taking the ratio between a scaler which counts the number of hardware triggers which take place when the computer in not busy and a scaler which counts the total number of hardware triggers. The chamber efficiency $C E$ is determined as the fraction of events which fall within the PID box and simultaneously satisfy the 'good chamber test' discussed in a previous section. The correction factor $C F$ is given by:

$$
C F=\frac{1}{\tau_{c} \cdot C E}
$$

Table 3.3 gives the values of the parameters discussed above as employed to obtain the normalized yields for the reactions of interest.

The efficiency parameters in a given angular gate obtained from the Monte Carlo simulation are quoted with the statistical errors associated with them. 
Table 3.3: Parameters employed to normalize experimental yields.

\begin{tabular}{|l|cc|cc|}
\hline & \multicolumn{2}{|c|}{${ }^{3} \mathrm{H}$} & \multicolumn{2}{c|}{${ }^{3} \mathrm{He}$} \\
\hline & normal & reverse & normal & reverse \\
\hline$\tau_{\mathrm{c}}$ & 0.865 & 0.886 & 0.886 & 0.889 \\
$C E$ & 0.733 & 0.742 & 0.601 & 0.611 \\
$E R 04$ & 3414901 & 3084917 & 9831786 & 8850427 \\
$\epsilon\left(\Delta \theta_{1}\right)$ & $0.9780 \pm 0.0014$ & $0.9450 \pm 0.0014$ \\
$\epsilon\left(\Delta \theta_{2}\right)$ & \multicolumn{2}{|c|}{$0.9890 \pm 0.0017$} & $0.9770 \pm 0.0017$ \\
\hline
\end{tabular}

The ratio between the cross sections, $R$ is calculated as:

$$
R=\frac{\left[J \cdot\left(Y_{n}^{\prime}+Y_{r}^{\prime}\right)\right]_{3_{H+\pi^{+}}}}{\left[J \cdot\left(Y_{n}^{\prime}+Y_{r}^{\prime}\right)\right]_{{ }_{3}+\pi^{0}}}
$$

We note that the yields $Y_{n, r}^{\prime}$ above are integrated over the full peaks (effectively $-40 \geq M M \leq 40 \mathrm{MeV})$.

The analyzing power is obtained as:

$$
A_{y 0}=\frac{1}{P_{B}} \cdot \frac{\left(Y_{n}^{\prime}-Y_{r}^{\prime}\right)}{\left(Y_{n}^{\prime}+Y_{r}^{\prime}\right)}
$$

The beam polarization was obtained from the average between the polarization values calculated with the quench method from the beam monitors ER02 and ER04. The average value of the polarization of the beam during this experiment was 0.840 .

The final distributions of the analyzing power as a function of the missing mass for the two reactions are given in Fig. 3.22. It should be noted that the deviant values of $A_{y^{0}}$ come from regions of $M M$ which have very little statistical weight.

In order to obtain the observable $\Delta A_{y o}$ the data were integrated over the missing mass intervals corresponding to stable values of the analyzing power. These are respectively $-4 . \mathrm{MeV}-20 . \mathrm{MeV}$ for ${ }^{3} \mathrm{H}$ and $-10 . \mathrm{MeV}-20 . \mathrm{MeV}$ for ${ }^{3} \mathrm{Ile}$. 

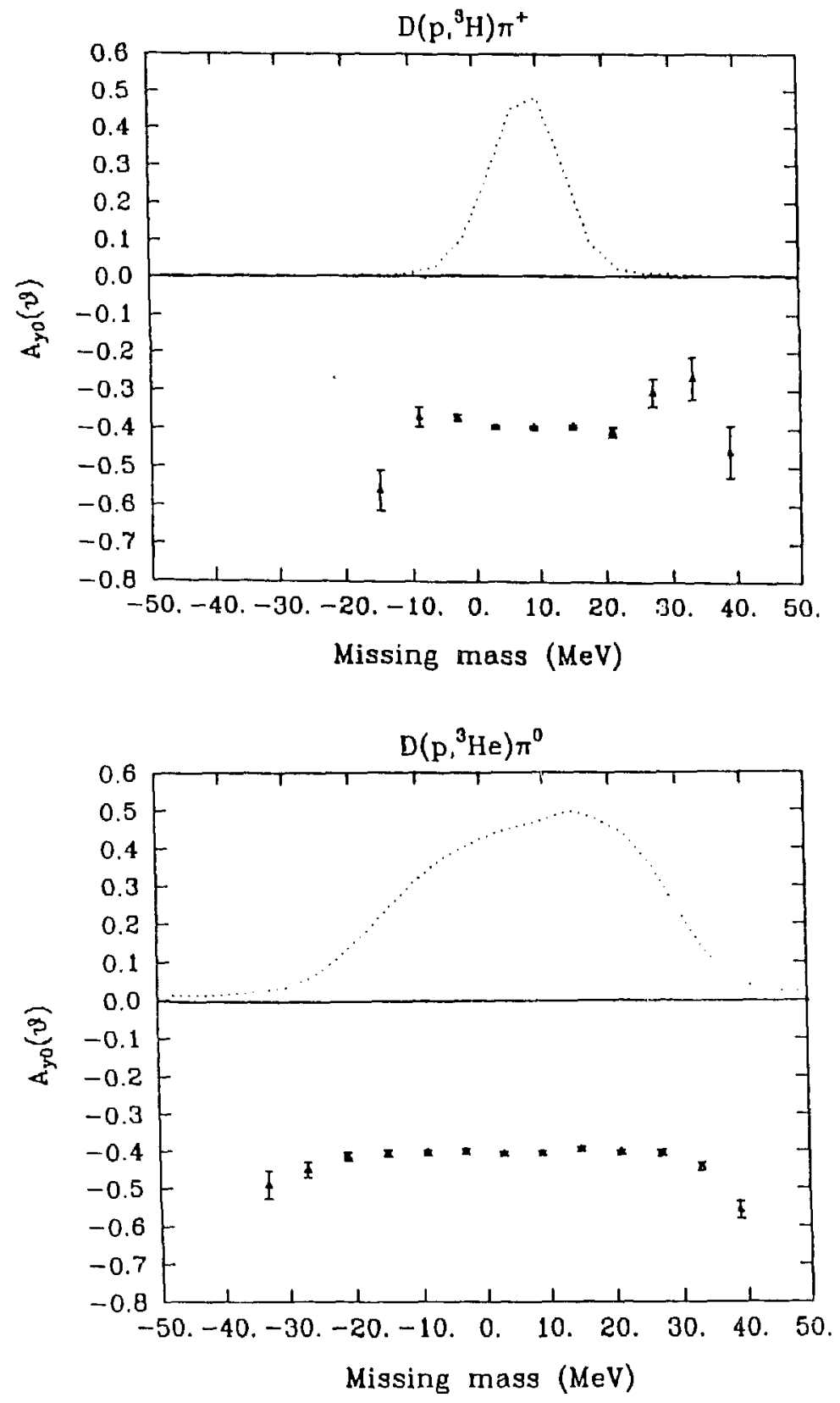

Figure 3.22: $A_{y 0}$ as a function of missing mass for the reactions $\vec{p}+d \rightarrow{ }^{3} H+\pi^{+}$ and $\vec{p}+d \rightarrow^{3}$ If $e+\pi^{0}$ (after background subtraction. The dotted curve indicates the missing mass shape from Fig. 3.20). 
3.7 Error analysis.

We will now examine the sources of systematic errors and evaluate their effects on the precision of the observables measured.

1. Beam Energy Variation.

The relative stability of the beam energy was determined by comparing the positions of the centroid of the missing mass peak corresponding to elastic scattering from deuterium in two runs taken respectively near the beginning and the end of the experiment. This difference was $\pm 0.250 \mathrm{MeV}$, which corresponds to a variation $\Delta T_{\text {beam }}= \pm 0.300 \mathrm{MeV}$. As mentioned before, at $\approx 700 \mathrm{MeV}$ and $\theta_{c m}(\pi)=130$ ' a change in the beam energy by $1 \mathrm{MeV}$ causes less than $0.5 \%$ change in the cross section. Therefore $\Delta T_{\text {beam }}= \pm 0.300 \mathrm{MeV}$ corresponds to less than $\pm 0.15 \%$ change in the cross section. This variation, already negligible, is further reduced, as far as the ratio $R$ is concerned, as data were taken in a 'cycling' mode, alternating between ${ }^{3} \mathrm{H}$ and ${ }^{3} \mathrm{He}$ detection approximately each 5 hours. Therefore fluctuations on the beam energy have on the average the same effect on the ${ }^{3} \mathrm{H}$ and ${ }^{3} \mathrm{He}$ cross sections and cancel out in $R$.

$\Delta A_{y 0}$ is not affected by this source of error as the analyzing power is defined as a ratio between cross sections and therefore is not sensitive to variations of their absolute value.

\section{Beam Position.}

No monitor of fluctuations of beam position at the target was available. However a quite sensitive monitor of the beam position $(\approx 2 \mathrm{~m}$ upstream of the target) is provided by the polarimeter which is equipped with $U_{p}-$ Down and LeftRight detectors. Changes on the geometrical factors $G_{\text {hor }}$ and $G_{\text {vert }}$ (defined in chapter 2) reflect the displacement of the beam spot in the horizontal and vertical plane respectively. A calibration in the changes of these geometrical factors with 


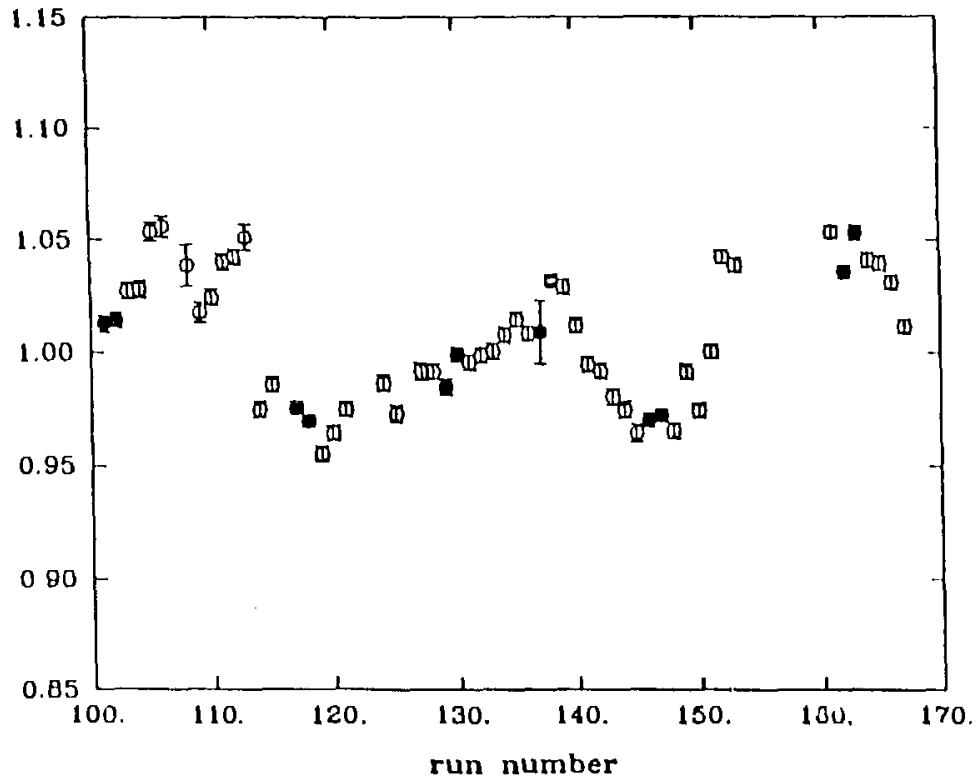

Figure 3.23: Measured geometrical factors $G_{\text {hor }}$ for Line-C polarimeter. The open circles correspond to ${ }^{3}$ He detection and the filled circles correspond to ${ }^{3} \mathrm{II}$ detection.

respect to the beam position has been done by the MP10 staff and the results are:

$$
\begin{aligned}
& \frac{d G_{\text {vert }}}{d y}=0.26 / \mathrm{cm}, \text { or reciprocally } 0.38 \mathrm{~mm} / \% \text { change in } G_{\text {vert }} \\
& \frac{d G_{h o r}}{d x}=0.43 / \mathrm{cm}, \text { or reciprocally } 0.22 \mathrm{~mm} / \% \text { change in } G_{h o r}
\end{aligned}
$$

Changes in beam position have two effects. The first is to change the eflective thickness of the target, according to Table 2.2 and the second is to change the geometrical acceptance of the spectrometer. During the course of our experincnt it was found that the geometrical factors $G_{\text {hor }}, G_{\text {vert }}$ changed by up to $\pm 5 \%$ about their mean values, as is shown in Fig. 3.23. This corresponds to up to \pm 1.2 min horizontal displacement and $\pm 1.9 \mathrm{~mm}$ vertical displacement. $\Lambda$ s Table 2.2 shows one millimeter horizontal displacement changes the target thickness by woll under $i \%$. However, this variation is of no consequence as far as the error in our determination of $R$ is concerned. What matters is the ratio of the average values 
of $G$ between ${ }^{3} \mathrm{H}$ and ${ }^{3} \mathrm{He}$ runs:

$$
\rho=\frac{\langle G\rangle_{3_{H}}}{\langle G\rangle_{\mathrm{s}_{\mathrm{H}}}}=\frac{\left(\sum_{i=1}^{N} E R 04_{i} \cdot G_{i} / \sum_{i=1}^{N} E R 04_{i}\right)_{{ }_{3_{H}}}}{\left(\sum_{j=1}^{N^{\prime}} E R 04_{j} \cdot G_{j} / \sum_{j=1}^{N^{\prime}} E R 04_{j}\right)_{s_{H_{e}}}}
$$

where the numerator represents a weighted average of the geometrical factor over the ${ }^{3} \mathrm{H}$ runs and the denominator represents a weighted average over the ${ }^{3} \mathrm{He}$ runs.

Because we alternated ${ }^{3} \mathrm{H}$ and ${ }^{3} \mathrm{He}$ runs most fuctuations effectively cancel out. For example for the horizontal displacement we obtain the average value:

$$
\rho_{\text {hor }}=\frac{\left\langle G_{\text {hor }}\right\rangle_{s_{H}}}{\left\langle G_{\text {hor }}\right\rangle_{\mathrm{s}_{e}}}=\frac{1.0024}{1.0074}=0.995
$$

The ratio $\rho_{\text {hor }}$ corresponds to a relative horizontal beam displacement of $\approx 0.1 \mathrm{~mm}$ or to relative target thickness changes well under $\pm 0.1 \%$. We have therefore made no correction for this effect. Because the beam spot at the target is a vertical line $\approx 3 \mathrm{~cm}$ in height, the effect of vertical displacement of the same order is even less. It also has been neglected.

$\Delta A_{y 0}$ is not affected by target thickness variations due to beam spot movements. Monte Carlo simulation shows that there is no change in spectrometer acceptance for such small displacements in beam position either.

\section{Beam Polarization.}

The uncertainty in the determination of the polarization of the beam produces an error in the difference of analyzing power. $A \pm 1 \%$ error in beam polariza$\operatorname{tion}^{80}$ gives an error in $\Delta A_{y 0}$ equal to $0.01 \cdot \Delta A_{y 0}=0.06 \times 10^{-3}$. This is completely negligible.

\section{Beam Integration}

In order to evaluate the effects of errors in beam integration on $R$, we can write:

$$
R=\frac{\left(C F_{N}\right)_{3_{H}}}{\left(C F_{N}\right)_{3_{3}}} \cdot \frac{\left(E R 04_{N}\right)_{3_{H}}}{\left(E R 04_{N}\right)_{3_{H}}} \cdot \frac{\left[\frac{J}{\epsilon(\Delta \theta)} \cdot\left(Y_{N}+\frac{C F_{R}}{C F_{N}} \frac{E R 04_{N}}{E R 04_{R}} Y_{R}\right)\right]_{{ }^{H}}}{\left[\frac{J}{\epsilon(\Delta \theta)} \cdot\left(Y_{N}+\frac{C F_{R}}{C F_{N}} \frac{E R 04_{N}}{E R 04_{R}} Y_{R}\right)\right]_{{ }_{3} H_{e}}}
$$


We can safely assume that $E R O 4_{n}=E R O 4_{r}$ and $C F_{n}=C F_{r}$, as the data were taken by alternating the sign of the beam polarization each three minutes and therefore relative luctuations were averaged out. As a consequence the two quantities within square brackets can be considered constant so that we can write:

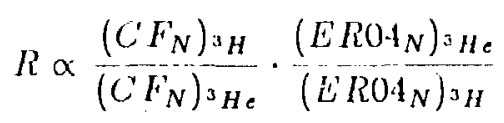

From this relationship we can deduce that:

$$
\left(\frac{\Delta R}{R}\right)_{E R 04}=\left[\left(\frac{\Delta E R 04}{E R 04}\right)_{3_{H e}}-\left(\frac{\Delta E R 04}{E R 04}\right)_{{ }^{3} H}\right]
$$

This shows that the absolute precision of the beam monitor $\triangle L R 04 / E R 04$ does not affect the error in $R$; only the difference during ${ }^{3} \mathrm{H}$ and ${ }^{3} \mathrm{He}$ measurements matters. In order to determine the reliability of the stability of the beam monitor ER04 with time we consider the ratio $(E R 04 / E R 02)$. ER02, the other beam integrator located in the scattering chamber, was known to have a small gas leak and therefore a monotonic drift in its gain. Therefore a linear least square fit has been made to the ratio $E R 04 / E R 02$ as a function of time. The standard error of this fit is found to be $0.15 \%$, which shows that there were no signilicant short-term fluctuations in ER04 (or ERO2).

The ratio $R$ is sensitive to long term drifts of the beam monitors employed. As Eq. (3.23) shows, if data for the two reactions had been taken in two separate long runs this source of systematic error would not be negligible. However, since we have alternated many ${ }^{3} \mathrm{II}$ and ${ }^{3} \mathrm{He}$ runs, the summed data are averaged over the same experimental conditions and no error due to beam integration survives. This argument also applies to the determination of $\Delta A_{y 0}$. Therefore no error associated with beam integration uncertainty has been attribuied to these observables.

5. Target density.

A possible sourco of error in the measurement of cross sections using a liquici target is a fluctuation of the target density produced by partial boiling of the liquid 
deuterium. An indication of the stability of the density is given by the target pressure. During our experiment the target pressure was inonitored continuously and had a stable value of $14.5 \mathrm{psi}( \pm \leq 0.05 \mathrm{psi})$

\section{Correction Factor $C F$.}

Uncertainties in the correction factor $C F$ are an important contributor to ihe final uncertainties in $\mathrm{R}$. The correction factor is the product of the computer livetime correction $1 / T_{c}$ and the chamber efficiency correction $1 / C E$.

The dependence of the relative error upon this parameter is again:

$$
\frac{\Delta R}{R}=\left[\left(\frac{\Delta C F}{C F}\right)_{{ }^{3} \mathrm{H}}-\left(\frac{\Delta C F}{C F}\right)_{{ }^{3} \mathrm{He}_{e}}\right]
$$

The livetime $\tau_{c}$ is determined as the ratio between two scalers which count respectively the nuĩuber of taped triggers and the total number of triggers. Therefore we can apply to it the same argument by which we demonstrated that no error is associated with the beam integrator. $A$ more careful analysis must be performed for the chamber efficiency $C E$, as it is determined from ratios of tests applied to data words associated with detectors operating at different voltage settings and detecting particles with different ionizing properties. Therefore the assumption of equal accuracy of the chamber efficiency correction can not be taken for granted. The statisuical error in $C E$ is not negligible ( $\pm 0.23 \%$ ). It contributes an error in $R$ equal to $\pm 0.33 \%$.

As the efficiency correction is an integral quantity, which averages out the differential distribution over the whole active $\Delta x_{f}$ region, there is an error introduced when it is applied to a restricted region within $\Delta x_{f}$. Moreover, as the $x_{f}$ distributions of the events corresponding to the angu.ur gates used in the analysis are not exactly the same for ${ }^{3} \mathrm{II}$ and a ${ }^{3} \mathrm{He}$ a corresponding error in the ratio can be introduced. An estimate of this source of error can be obtained with the information from the Monte Carlo simulation. As mentioned before, the program reproduces rather accurately the distribution of events relative to the coordinates 
at the front plane. The $x_{j}$ distribution, obtained under the assumption of uniform efficiency of the detectors within their geometrical boundaries, agrees rather well with the experimental one in the central region $(-10$. to $+10 \mathrm{~cm})$. However it exhibits a slower fall-off at the two edges of the vertical acceptance, This effect can be attributed to non-uniform efficiency of the drift chambers or to a less efficient transmission of peripheral rays (for instance, because of scattering at the walls of the spectrometer vacuum chamber). Fig. 3.24 shows the $x_{f}$ distribution for the events corresponding to ${ }^{3} \mathrm{H}$ and ${ }^{3} \mathrm{He}$ detection for the gates $\Delta \theta_{1}$ and $\Delta \theta_{2}$ respectively. It can be seen that the ${ }^{3} \mathrm{H}$ and ${ }^{3} \mathrm{He}$ spectra have a different distribution and therefore a relative error can be introduced in applying only an integral chamber efficiency correction to them. In order to get an estimate of this error, we used the differential efficiency distribution obtained by comparison between the experimental $x_{f}$ spectrum and the corresponding one generated by the Monte Carlo simulation for the continusm run $\mathrm{d}\left(\mathrm{p}, \mathrm{p}^{\prime}\right) \mathrm{X}$. In each region the ratio between the area under the experimental $\Delta x_{f}$ bir and the corresponding Monte Carlo simulation bin has been taken $\left(\epsilon_{r}=1\right.$ in the central region). The efficiency in the central region has been obtained by inverting the relationship:

$$
C E=\frac{1}{6} \sum_{n=1}^{6} \frac{Y_{i}^{s i m}}{Y_{i}^{\text {exp }}} \cdot \epsilon_{c}
$$

where:

$C E$ is the experimental chamber efficiency correction

$Y_{i}^{s i m}$ is the partial yield in the $\mathrm{i}^{\prime}$ th $\mathrm{xf}$ interval for the spectrum generated by the Monte Carlo simulation.

$Y_{i}^{\text {exp }}$ is the corresponding partial yield for the experimental spectrum, $\epsilon_{c}$ is the efliciency in the central region.

Then the relative error between the corrected yield calculated by weighting the partial yiclds in $\Delta x_{i}$ with their own chamber efficiency cocflicient and the 
corrected yields calculated by applying the overall $C E$ correction has been evaluated for the two reactions studied in our experiment. This caiculation has given a $0.61 \%$ error in $R$ for gate $\Delta \theta_{1}$ and $0.84 \%$ in $R$ for gate $\Delta \theta_{2}$. This source of error has no effect on $\Delta A_{y 0}$, as this observable does not depend upon the absolute value of the efficiency correction.

7. Background Subtraction.

A major source of error is the uncertainty in the procedure of backgroind subtraction. In order to evaluate the sensitivity of the $R$ and $\Delta A_{y 0}$ to this source of systematic error, background distributions increased by $\pm 10 \%$ were gersated and the corresponding corrected yields were used in calculating $\Delta A_{\nu 0}$ and $R$.

The effect of the background subtraction uncertainty on $R$ is found to be $\pm 0.68 \%$ for the angular gate $\Delta \theta_{1}$ and $\pm 0.61 \%$ for the angular gate $\Delta \theta_{2}$.

The corresponding uncertainty in $\Delta A_{y 0}$ is found to be $\pm 1.8 \times 10^{-3}$ for both gates.

8. Summary.

Table 3.4 summarizes the various sources of error mentioned before. The errors have been combined in quadrature to determine the total systematic error attributed to the observable $R$. The error in $\Delta A_{y 0}$ is essentially the one produced by background subtraction, i.e. $\pm 1.8 \times 10^{-3}$.

Table 3.4: Surnmary of error analysis.

\begin{tabular}{|c|c|c|c|}
\hline \multirow{2}{*}{ Source of error } & \multicolumn{2}{|c|}{$\%$ in $R$} & $\Delta \bar{A}_{y n}$ \\
\hline & $\triangle \bar{\theta}_{1}$ & $\bar{\Delta} \overline{\theta_{2}}$ & $\Delta \bar{\theta}_{1}$ and $\Delta \bar{\theta}_{2}^{-}$ \\
\hline Juen energy variation & \multicolumn{2}{|c|}{$<0.15$} & \\
\hline Hearr position variation & \multicolumn{2}{|c|}{$<0.1$} & \\
\hline Frror in beam polarization & \multirow{3}{*}{\multicolumn{2}{|c|}{$\begin{array}{c}<0.15 \\
\approx 0\end{array}$}} & $0.06 \times 10^{-3}$ \\
\hline Firror in beam integration & & & \\
\hline 'larget Inensity & & & \\
\hline Chamber efliciency (statistical accuracy) & $0 . \overline{3} \overline{3}$ & $0 . \overline{3} \overline{3}$ & \\
\hline (Mamber elliciency (differential) & 0.61 & 0.84 & \\
\hline Mackpround subtraction & 0.68 & 0.61 & $1.8 \times 10^{-9}$ \\
\hline Total & $0.9 \overline{7}$ & 1.09 & $1.8 \times 10^{-3}$ \\
\hline
\end{tabular}



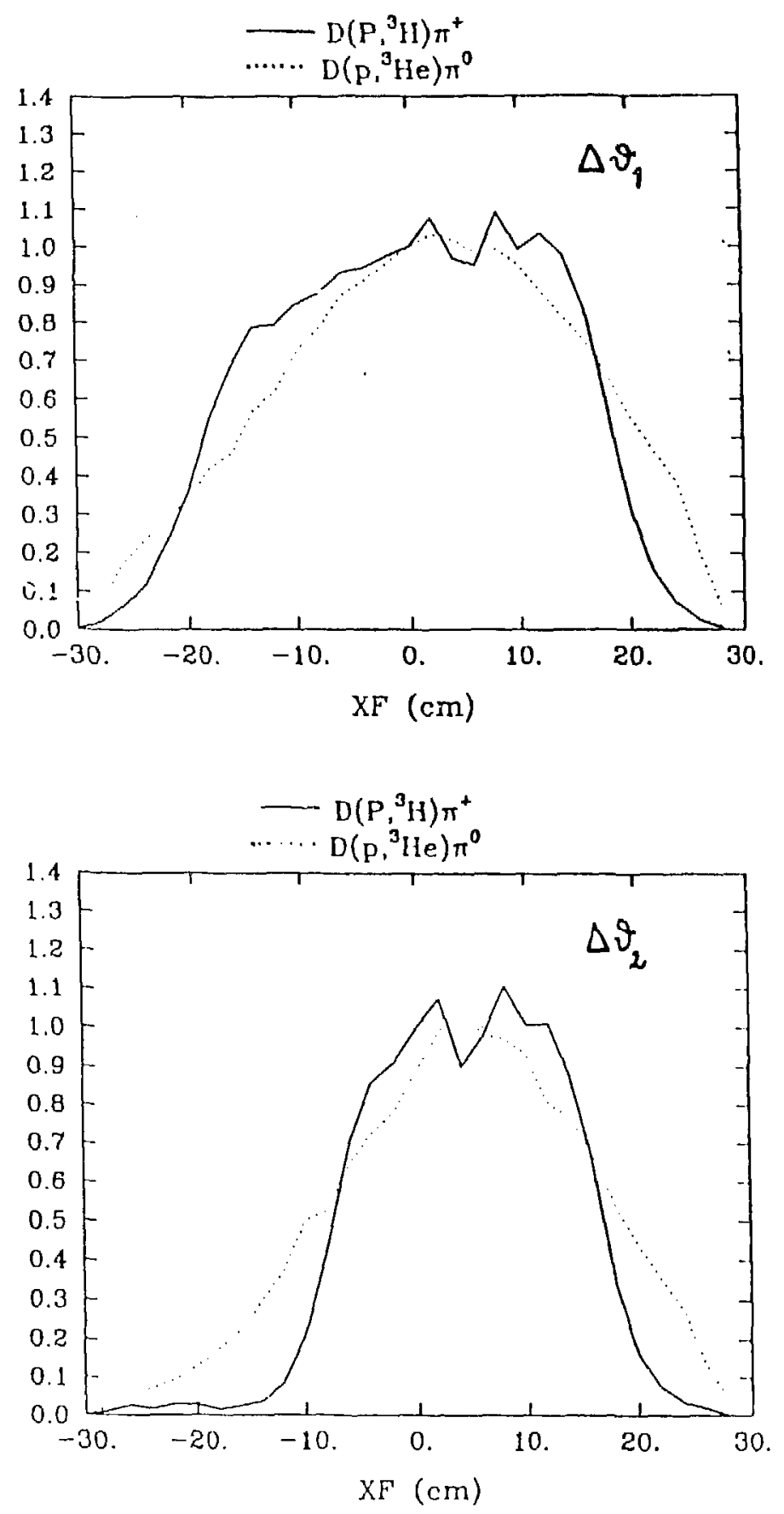

Figure 3.24: $x_{f}$ distribution of events for ${ }^{3}$ II and ${ }^{3}$ He within the gates $\Delta \theta_{1}$ and $\Delta \mathrm{O}_{2}$. 


\subsection{Discussion of Experimental Results.}

Tables 3.5 and 3.6 summarize the results for the difference in analyzing power and the ratio between differential cross sections. The average values of $\theta_{\pi}^{c m}$ and the range $\Delta \theta_{\pi}^{c m}$ over which the integration is performed are given both for ${ }^{3} \mathrm{H}$ and ${ }^{3}$ He. The average values of $\theta_{\pi}^{\mathrm{cm}}$ differ by less than $.05 \%$ in the two reactions compared. This suggests that no difference in kinematical conditions affects these results. It should be noted that the angular regions of integration are slightly different (by $\approx 0.5 \%$ ) for ${ }^{3} \mathrm{II}$ and ${ }^{3}$ Ile. The ratio $R$ has been corrected for this effect by normalizing the cross sections to the width of the angular integration region.

Recently some claims have been made that the correct variable at which to perform the comparison is the 4 -momentum transfer $t$ between the proton and the pion. The average values of $t$ which are given in Table 3.5 correspond to a difference of less than $.5 \%$ between the two reactions. Since the two angular gates have approximately $1 \%$ difference in average $t$, we can easily see that the $0.5 \%$ difference in $t$ between the two reactions produces differences less than the statistical errors.

It is worthwhile to compare the results of the present experiment with the earlier results in published literature. The results for the ratio of cross sections, $\mathrm{R}$ have already been summarized in Table 1.7. We have also given the critique for individual experiments in Chapter 1. As stated earlier, most of the experiments quote only statistical errors. Little or no discussion of systematic errors is given. As far as statistical errors alone are concerned, our result

$$
R=2.193 \pm 0.007 \pm(0.024)
$$

has only $\pm 0.3 \%$ errors as compared to $\pm 2.5 \%$ (Harting et al. ${ }^{71}$ ) to $\pm 17 \%$ errors (Silverman et $a .^{73}$ ) associated with other experiments. Even after our estimate of $\pm 1.1 \%$ systematic errors is included, our result for $\mathrm{R}$ represents a factor five to 
ten improvement over all earlier experiments.

There are no earlier measurements of $\Delta A_{\nu 0}$ for the reactions studied by us. The precision of our experimental result

$$
-\Delta A_{\text {vo }}=0.0065 \pm 0.0040 \pm(0.0018)
$$

can only be compared to a completely different experiment, i.e., the most recent experiment to test charge symmetry in $n p$ elastic scattering ${ }^{63}$. In this extremely elaborate experiment

$$
\Delta A_{y 0}=0.0034 \pm 0.0017 \pm(0.0008)
$$

was measured. It is extremely gratifying that we have reached almost an equal level of precision in our experiment. 
Table 3.5: Summary of Experimental results(I). The errors are statistical only.

\begin{tabular}{|c|c|c|c|c|c|c|c|c|}
\hline \multirow[b]{2}{*}{ Gate } & \multicolumn{4}{|c|}{$\mathrm{d}\left(\mathrm{p},{ }^{3} \mathrm{H}\right) \pi^{+}$} & \multicolumn{4}{|c|}{$\mathrm{d}\left(\mathrm{p},{ }^{3} \mathrm{He}\right) \pi^{0}$} \\
\hline & $\Delta \theta^{\circ}$ & $\left\langle\theta_{\pi}^{\mathrm{cm}}\right\rangle$ & $\begin{array}{c}-\langle t\rangle \\
(\mathrm{GeV} / \mathrm{c})^{2}\end{array}$ & $A_{y 0}(\theta)$ & $\Delta \theta^{\circ}$ & $\left\langle\theta_{\pi}^{c m}\right\rangle$ & $\begin{array}{c}-\langle t\rangle \\
(\mathrm{GeV} / \mathrm{c})^{2}\end{array}$ & $A_{y 0}(\theta)$ \\
\hline 1. & 6.46 & 130.02 & 0.559 & $0.3923=.0023$ & 6.43 & 130.08 & 0.563 & $0.4005 \pm .0028$ \\
\hline 2. & 4.29 & 130.51 & 0.563 & $0.3937 \pm .0027$ & 4.27 & 130.52 & 0.567 & $0.3986 \pm .0033$ \\
\hline & \multicolumn{3}{|c|}{ average } & $0.3930 \pm .0025$ & \multicolumn{3}{|c|}{ average } & $0.3996 \pm .0031$ \\
\hline
\end{tabular}

Table 3.6: Summary of experimental results (II). On each quantity, the first errors are statistical, and the second errors (in parentheses) are systematic.

\begin{tabular}{|c|c|c|c|c|c|}
\hline Gate & $\begin{array}{c}-\langle t\rangle\left({ }^{3} \mathrm{H}\right) \\
(\mathrm{GeV} / \mathrm{c})^{2}\end{array}$ & $\begin{array}{c}-\langle t\rangle\left({ }^{3} \mathrm{He}\right) \\
(\mathrm{GeV} / \mathrm{c})^{2}\end{array}$ & $R$ & $-\Delta A_{y 0}$ & $-100 \cdot \Delta A_{y 0} /\left\langle A_{y 0}\right\rangle$ \\
\hline 1. & 0.559 & 0.563 & $2.195 \pm .006 \pm(.021)$ & $0.0082 \pm .0036 \pm(.0018)$ & $2.05 \pm .900 \pm(.45)$ \\
2. & 0.563 & 0.567 & $2.190 \pm .007 \pm(.024)$ & $0.0049 \pm .0044 \pm(.0018)$ & $1.23 \pm 1.10 \pm(.46)$ \\
\hline Av. & \multicolumn{2}{|c|}{0.563} & $2.193 \pm .007 \pm(.024)$ & $0.0065 \pm .0040 \pm(.0018)$ & $1.63 \pm 1.00 \pm(.46)$ \\
\hline
\end{tabular}




\section{Chapter 4}

\section{Theoretical analysis and conclusions.}

As we mentioned in chapter 1, a proper interpretation of our experimental results can only be done if one has a successful theory of pion production in pd collisions. We will describe several attempts to develop such a theory below.

In absence of a theory of pion production one is forced to take a more limited approach to the interpretation of our results. Such approaches are based on the premise that although one may not be able to explain the course of differential cross sections for either of our reactions, one can still make reasonable calculations of the differences or ratios of the cross sections for the two reactions. This approach is obviously more valid for the geometrical and kinematical aspects of the two reactions. We begin with the description of one such calculation.

\subsection{The calculation of Köhler.}

Köhler ${ }^{91}$ has made a detailed analysis of the corrections to the ratio of cross sections

$$
R=\sigma(\theta)\left[{ }^{3} \mathrm{H}+\pi^{+}\right] / \sigma(\theta)\left[{ }^{3} \mathrm{He}+\pi^{0}\right\}
$$

which result from the kinematical differences between the two reactions (originating irom $\pi^{+}, \pi^{0}$ mass differences) and the external electromagnetic corrections (wave function differences and Coulomb distortions).

While Köhler makes no attempt to calculate cross sections in his paper, his 


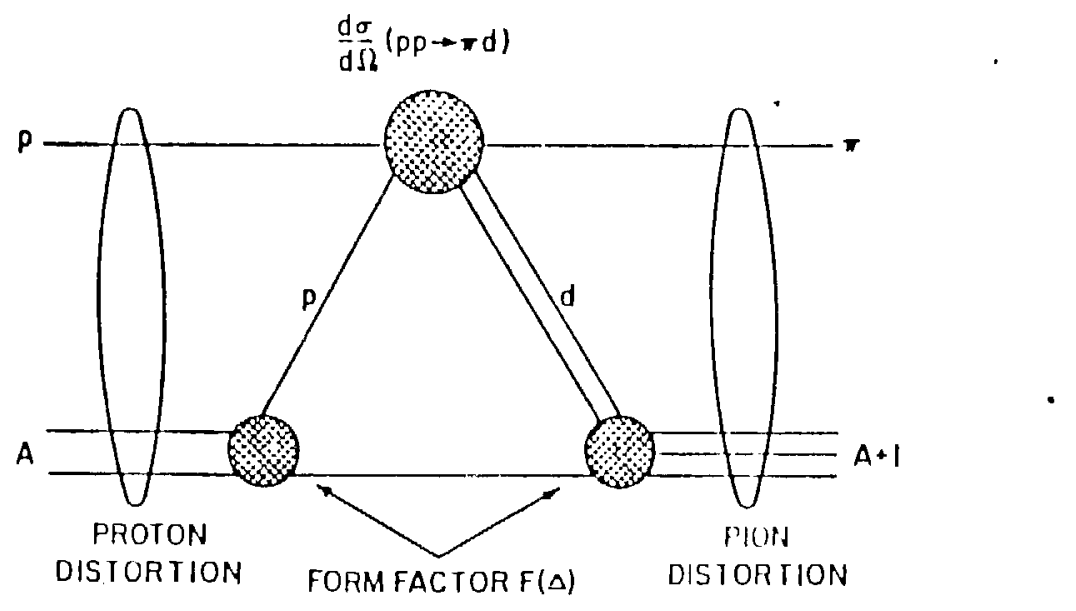

ligure 1.1: 'The Impulse approximation for the $p d \rightarrow t \pi^{+}$reaction (from Ref. 94).

model for the reaction essentially follows the impulse approximation of Ruderman ${ }^{67}$ and IBludman. ${ }^{92}$

'The basic premise of the impulse approximation is that the incident proton interacts essentially with one of the nucleons of the deuteron. $\Lambda \mathrm{pp} \rightarrow \mathrm{d} \pi^{+}$or pn $\rightarrow, 1 \pi^{0}$ reaction takes place, and the resulting deuteron forms ${ }^{3} \mathrm{II}$ or ${ }^{3} \mathrm{IJe}$ with the spectator nucleon of the deuteron. This is illustrated in Fig. 4.1. In this model the dilferential cross sections are given by

$$
\frac{\mathrm{d} \sigma}{\mathrm{d} \Omega}(0)=\mathrm{K} \cdot \mathrm{g} \cdot|F(\Delta)|^{2} \cdot \frac{\mathrm{d} \sigma}{\mathrm{d} \Omega}(\mathrm{p} \mathrm{N} \rightarrow \mathrm{d} \pi,\langle 0\rangle,\langle\mathrm{E}\rangle)
$$

IIere $\mathrm{K}$ is a kinematical factor, $\mathrm{g}$ is a numerical factor arising from proper inclusion of spin, isospin and antisymmetrization effects, $F(\Delta)$ is a form factor evaluated at a momentum transfer $\Delta$, and the $\mathrm{pp} \rightarrow \mathrm{d} \pi$ cross section is evaluated at an average two body energy and angle.

1. Corrections in $\mathrm{R}$ due to wavefunction differences.

The ratio of the cross sections

$$
R \equiv \frac{\mathrm{d} \sigma\left(\mathrm{pd} \rightarrow{ }^{3} \mathrm{H} \pi^{+}\right)}{\mathrm{d} \sigma\left(\mathrm{pd} \rightarrow{ }^{3} \mathrm{Ie} \pi^{0}\right)}=\frac{\mathrm{K}^{+} \mathrm{g}^{+}|\mathrm{F}(\Delta)|^{2} \sigma\left(\mathrm{pp} \rightarrow \mathrm{d} \pi^{+}\right)}{\mathrm{K}^{0} \mathrm{~g}^{0}\left|\mathrm{~F}^{0}(\Delta)\right|^{2} \sigma\left(\mathrm{pn} \rightarrow \mathrm{d} \pi^{0}\right)}
$$

If, for the moment, we assume that the $\pi^{+}$and $\pi^{0}$ masses are equal, then $\mathrm{K}^{+}=\mathrm{IK}^{0}$ 
and $\mathrm{g}^{+}=\mathrm{g}^{0}$. Since $\sigma\left(\mathrm{pp} \rightarrow \mathrm{d} \pi^{+}\right)=2 \sigma\left(\mathrm{pn} \rightarrow \mathrm{d} \pi^{0}\right)$, the simple prediction $R=2$ acquires a correction factor $=\left|\mathrm{F}^{\dagger}\right|^{2} /\left|\mathrm{F}^{0}\right|^{2}$. It is this correction factor due to the wave fuction differences which Köhler finds to be most important.

Köhler defines the form factor $F(\Delta)$ as

$$
\mathrm{F}(\Delta)=\int \psi_{3}(r x x) \psi_{\mathrm{d}}(x) \exp (i \vec{x} \cdot \vec{\Delta}) d \vec{x}
$$

where $4^{\prime} \cdot 3$ is the three body $\left({ }^{3} \mathrm{II}\right.$ or $\left.{ }^{9} \mathrm{He}\right)$ wavefunction, and the mometum transfor in the elenentary $p p \rightarrow d \pi$ reaction is

$$
\ddot{\Delta}-(\vec{k} / 2) \cdots(q / 3)
$$

where $\vec{k}$ and $q$ are center of mass momenta of the incident proton and the outgoing pion. For relatively simplified choices of wave functions for the three-body systems and the denteron, Köhler is able to obtain an analytic expression for $F(\Delta)$

$$
F(\Delta)=\left(\frac{1}{(\beta+\alpha)^{2}+\Delta^{2}}-\frac{1}{(\gamma+\alpha)^{2}+\Delta^{2}}\right) \cdot N_{3} .
$$

Ilere $10.229 \mathrm{fm}^{-1}$ and $\gamma=1.371 \mathrm{fm}^{-1}$ are parameters of the Hulthén wave function fur the dentren, the c's are related to the separation energy of a neutron from ${ }^{3}$ Il and that of a proton for ${ }^{3}$ Ile $\left(\alpha^{+}=0.907 \mathrm{fm}^{-1}, \alpha^{0}=0.890 \mathrm{fm}^{-1}\right)$, and $N_{3}$ are the overall normalization constants for the ${ }^{3} \mathrm{II}$ and ${ }^{3} \mathrm{He}$ wave functions. According to Köhler $\left.\mid N\left({ }^{3} \mathrm{H}\right) / N\left({ }^{3} \mathrm{H} \mathrm{c}\right)\right]^{2} \equiv\left[N^{+} /\left.N^{0}\right|^{2}=1.00\right.$.

Köhler evaluated the correction factor for $T_{\mathrm{p}}=600 \mathrm{MeV}$ and found it to be weakly angle dependent, ranging from $+5.9 \%$ to $+8.3 \%$. We have repeated Köhler's calculation for our kinematical conditions, i.c., $T_{\mathrm{p}}=730 \mathrm{MeV}, \theta_{\mathrm{cm}}=130^{\circ}$, incul find it to be equal to $15.3 \%$.

Köhler estimated an additional $0.6 \%$ correction due to $\mathrm{T}=3 / 2$ admixtures in the 3 Ile wavefunction and $1 \%$ correction due to the inclusion of the hard core in the nucleon nucleon interaction. Thus using Köhler estimates for these corrections, we get the net correction factor for our experiment due to wave function differences to be $+6.9^{\circ} \%$ 
2. Phase space differences.

Due to the $\pi^{+}, \pi^{0}$ mass difference the relativistic phase space for the two reactions is different.

$$
\begin{aligned}
& P S\left(\pi^{+}\right)=q_{\pi^{+}} \frac{E_{H} E_{\pi^{+}}}{\left(E_{\pi^{+}}+E_{H}\right)} \\
& P S\left(\pi^{0}\right)=q_{\pi^{u}} \frac{E_{H e} E_{\pi 0}}{\left(E_{\pi^{0}}+E_{H e}\right)}
\end{aligned}
$$

where $q_{\pi}$ refers to the pion momentum and $E$ represents the total energy of particle identified by the corresponding subscript.

The ratio $P S\left(\pi^{+}\right) / P S\left(\pi^{0}\right)=0.995$ in our case, i.e. the correction is $-0.5 \%$.

3. Correction due to $\mathrm{q}_{\pi}$ and $\theta_{\pi}$ differences.

If the two reactions are measured at the same laboratory angle, because of the differences in $\pi^{+}$and $\pi^{0}$ masses both the momentum $q_{\pi}$ and $\theta_{c m}(\pi)$ are different for the two reactions. In order to correct for the differences one has to know the variation of the differential cross sections both with respect to incident cnergy and outgoiing pion angle. Köhler estimated a $+3.58 \%$ correction due to these effects for the experiment of Harting et al. In our experiment we have made the angular cuts in such a manner as to minimize both the differences in $q_{\pi}$ and in $\theta_{\mathrm{cm}}(\pi)$ to $\leq 1.0 \%$. As mentioned earlier (in section 1.8), we estimate that these corrections lead to entirely negligible corrections in our experiment $(<.1 \%)$.

4. Corrections due Coulomb distortion and Impulse approximation.

Köhler argues that both are negligible $(\leq 1 \%)$.

To summarize, the 'external' corrections considered by Köhler amount to $\approx 6.4 \%$ in our experiment. In other words, with these external corrections only, ue expect $R=2.19$.

It is worth noting the since Köhlcr's original calculation (1960) great progress has been made in the study of few-nucleon systems. In particular, excellent wave functions are now available ${ }^{93}$ for the deuteron, ${ }^{3} \mathrm{II}$ and ${ }^{3} \mathrm{He}$. The three-body wave 
functions are now available ${ }^{93}$ for the deuteron, ${ }^{3} \mathrm{H}$ and ${ }^{3} \mathrm{He}$. The three-body wave functions are based on Faddeev calculations including Coulomb forces. However, generally the greatest attention has been paid to asymptotic aspects of these wave functions. In our reactions larg momentum transfers are involved and the wave function problem is much more complex. Nevertheless, it would be very instructive to make a calculation similar to Köhler's with the best wave functions available today. Hopefully, with such a calculution one would not have to resort to hand-waving arguments about effects of hard-core, and the d-state effects (neglected by köhler) would be included in a natural manner. One would also hope that Coulomb distortion effects can also be taken into account in a more satisfactory manner. Until such a calculation is available we should consider the above estimate of the overall external correction factor as essentially qualitative.

We note that Köhler has considered no 'internal' electromagnetic corrections. These arise due to the differcnces in the masses of the different charge pions which are exchanged and the different charge $\Delta$ 's $\left(\Delta_{33}\right)$ which may occur as intermediate states. 'lo shed some light on these corrections we have to consider more sophisticated theoretical calculations. 


\subsection{Theories of pion production.}

1. Calculation of Fearing. ${ }^{94}$

Fearing has improved on the impulse approximation theory in many ways to calculate the differential cross sections for the reaction $\mathrm{p} d \rightarrow^{3} \mathrm{II} \pi$.

1. Proton and pion distortions are included via an eikonal approximation which requires as inpui the nuclear shape and the elementary $\pi \mathrm{N}$ and $\mathrm{N} N$ scattering cross sections, thus making the model into a distorted wave impulse approximation (DWIA).

2. A number of technical improvements involving spin, antisymmetrization and better kinematics have been made.

3. Better wavefunctions, which reproduce electromagnetic form factors have been used. $\mathrm{D}$-state component of the deuteron wave function is included.

4. Better energy choice for $\mathrm{pp} \rightarrow \pi \mathrm{d}$ amplitude is made.

These improvements lead to mixed results. Below $T_{\mathrm{p}}=500 \mathrm{MeV}$ the shape of the experimental differential cross sections is well reproduc 1 , although the predicted absolute values are approximately factor two too small at all angles. With increasing energy the discrepancy with experimental data becomes worse, especially at back angles. By $T_{\mathrm{p}}=800 \mathrm{MeV}$ the forward angle cross sections are almost a factor 3 too small and the back-angle cross sections are predicted to be more than a factor 20 too small. This is illustrated in Fig. 4.2 where the experimental data from Ref. 90 are also shown.

Fearing has discussed several possible reasons for the failure of these calculations at $T_{\mathrm{p}}>500 \mathrm{MeV}$. The most important of these is the neglect of all but $\pi \mathrm{d}$ intermediate states. In the elementary pion production process the $\mathrm{pp} \rightarrow \pi \mathrm{d}$ channel certainly dominates at threshold $(\approx 288 \mathrm{MeV})$. Howeve' at $500 \mathrm{MeV}$ the three body channel pp $\rightarrow \pi^{+} p n$ becomes of equal strength, and at $800 \mathrm{MeV}$ it 


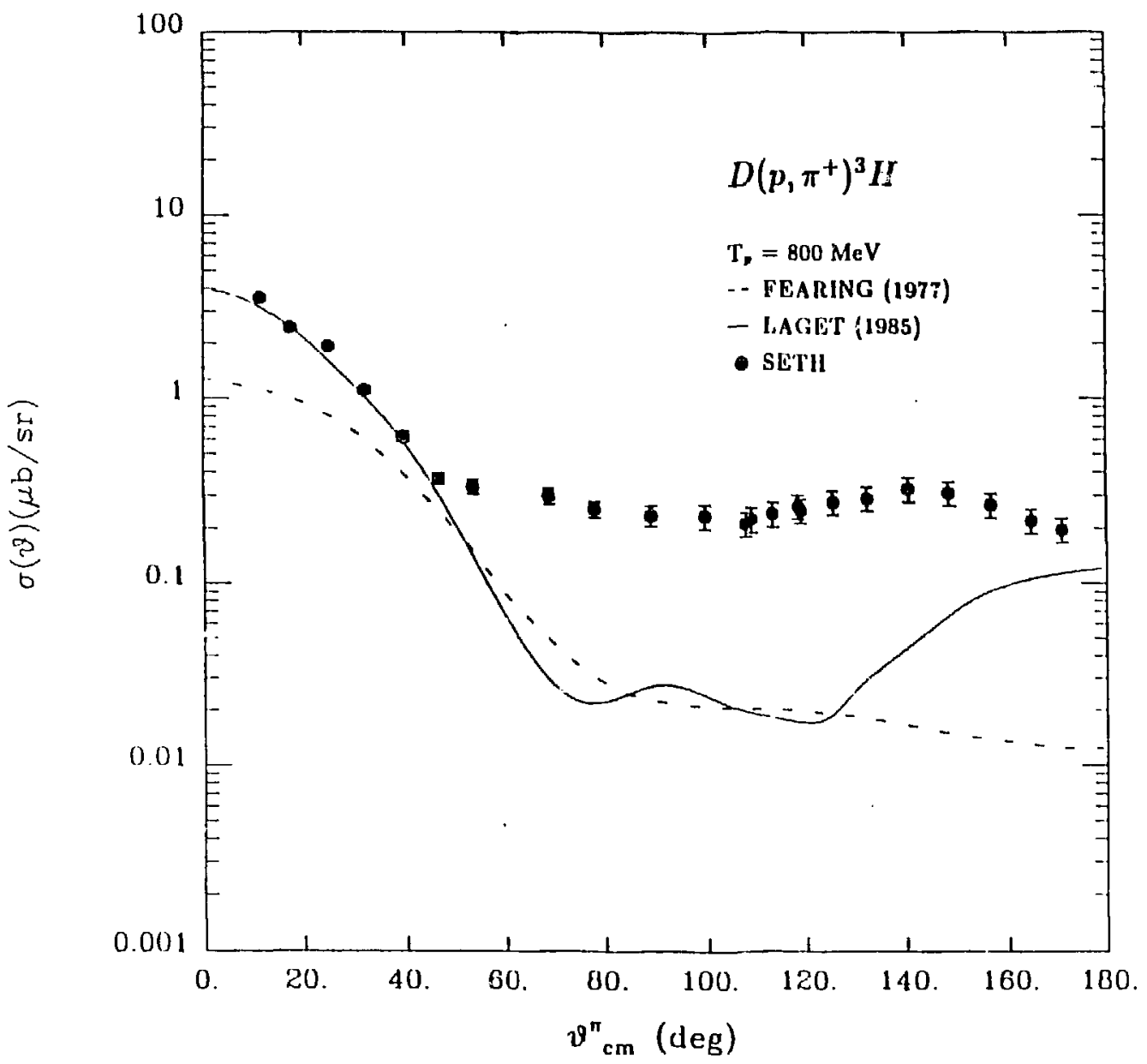

Figure 4.2: Differential cross sections for the $p d \rightarrow t \pi^{+}$reaction at $800 \mathrm{MeV}$. The experimental data are from Ref. 90. The theoretical curves are from Refs. 94 and 98. 
accounts for more than $90 \%$ of the single pion production cross section. Certainly the neglect of that part of the pn $\pi^{+}$production in which the pn pair emerges in the nearly bound ${ }^{1} S_{0}$ state is not justified.

The second important assumption is the factorization represented by Eq. 4.26, where the $\mathrm{pp} \rightarrow \mathrm{d} \pi$ cross section is factored out of the form factor integral $\mathrm{F}(\Delta)$. This is only justified when the $\mathrm{pp} \rightarrow \mathrm{d} \pi$ amplitude does not vary rapidly. At the resonance, $T_{\mathrm{p}} \approx 600 \mathrm{MeV}$, this may not be a good approximation, as has been pointed out by Green et al. ${ }^{95}$

To summarize, although Fearing's work represents definitive improvement over the earlier work of Ruderman, ${ }^{67}$ Bludman $^{92}$ and Köhler ${ }^{91}$, it fails badly in our domain of interest. Further, Fearing does not study the reaction pd $\rightarrow{ }^{3} \mathrm{He} \pi^{0}$ and is therefore not able to help us with Coulomb differences in $R$. Fearing makes no caiculation for $A_{y 0}(\theta)$.

\section{Calculations of Green and collaLorators. ${ }^{95-97}$}

Green and Niskanen ${ }^{96}$ achievcd considerable success in describing the elementary reaction

$$
\mathrm{pp} \rightarrow \mathrm{d} \pi^{+}
$$

in a coupled-channel isobar model. Following this success, Green and collaborators ${ }^{95}$ attempted to describe the $\mathrm{pd} \rightarrow \mathrm{t} \pi^{+}$reaction in terms of these same configurations, as illustrated in Fig. 4.3.

Early attempts with this model, in which one relates $\mathrm{pp} \rightarrow \mathrm{d} \pi^{+}$amplitudes to $\mathrm{pd} \rightarrow \mathrm{t} \pi^{+}$amplitudes (not cross sections, as is done in the approach of Fearing), had poor success. However the most recent calculations ${ }^{97}$ show considerably better success with the use of Faddeev wave functions and several technical improvements. As shown in Fig. 4.4 differential cross sections at all angles are reproduced quite well at $T_{\mathrm{p}}=400 \mathrm{Mev}$. Above $400 \mathrm{MeV}$ the agreement is reasonably good at forward angles and quite poor at back angles. By $600 \mathrm{MeV}$ the predicted back-angle cross sections are almosı a factor 50 too small. No cross 

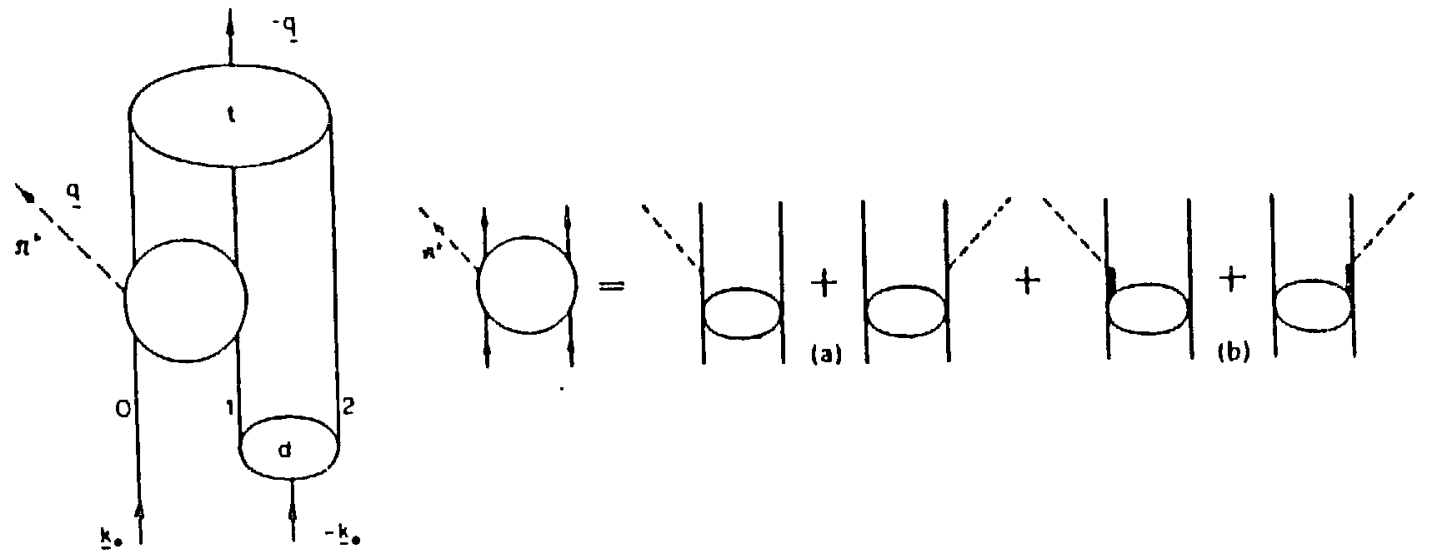

Figure 4.3: The basic pion production mechanism considered by Green and collaborators. The bubble in the diagram on the left is "expanded" on the right to show all the contributions.
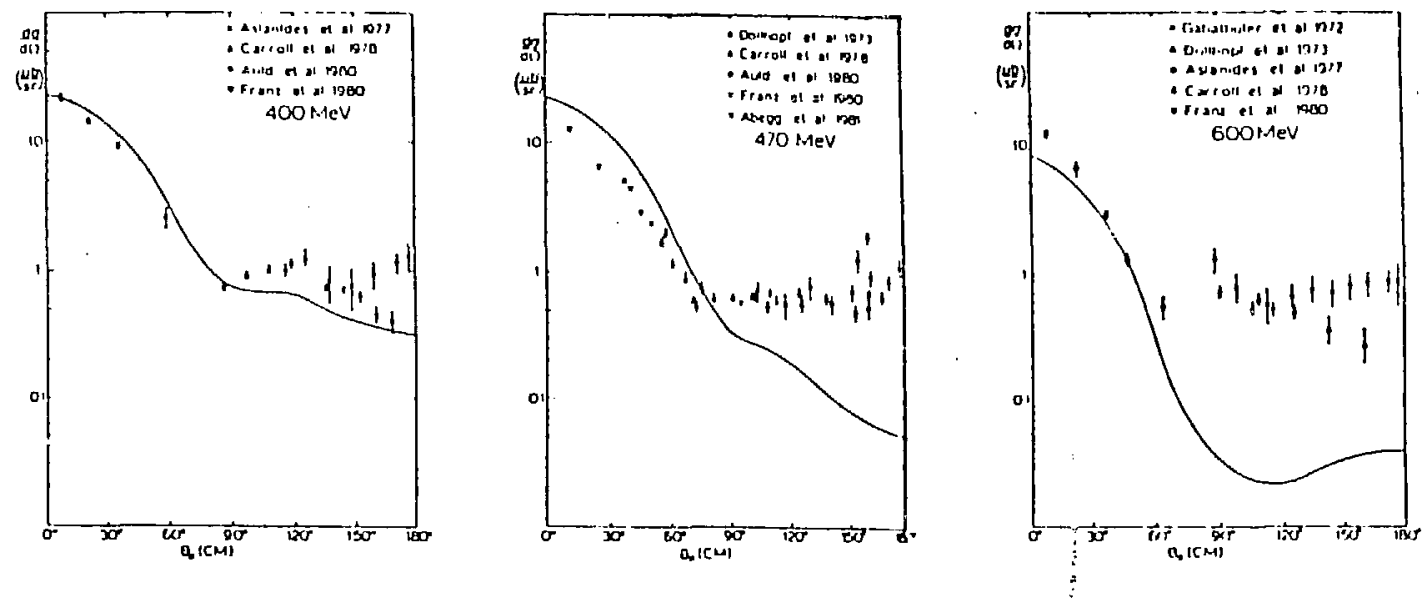

Figure 4.4: $p d \rightarrow t \pi^{+}$differential cross section predictions from the calculations of Green and collaborators (from Ref. 97). 
section calculations have been reported for $800 \mathrm{MeV}$. No $A_{y 0}(\theta)$ calculations have been made.

In summary, the model of Green and collaborators is as successful as that of Fearing at $T_{\mathrm{p}} \leq 400 \mathrm{MeV}$. At higher energies it suffers from the same malady as the Fearing model, only somewhat worse. Its cross sections fall far too rapidly at the back angles. It is therefore little consolation that this model is a microscopic one while the model of Fearing is essentially a phenomenological one. In any case, this model also promises little help in solving the problem posed by our experiment.

\section{Calculation of Laget and LeColley. ${ }^{98}$}

This is the most recent calculation. It makes substantial departures from previous efforts and will therefore be described in some detail.

At intermediate energies, the formation of the $\Delta_{33}$ resonance in the s-channel is considered to be the dominant contribution to the cross section for the reactions $p+p \rightarrow d+\pi^{+}$and $p+d \rightarrow{ }^{3} \mathrm{H}\left({ }^{3} \mathrm{Ile}\right)+\pi^{+}\left(\pi^{0}\right)$. Other processes play a significant role, particularly in polarization observables which are sensitive to interference terms between different amplitudes contributing to the reaction studied.

The amplitudes calculated in this model are illustrated in Fig. 4.5.

For clarity sake, the discussion of the various amplitudes will refer to the reaction $p+d \rightarrow{ }^{3} \mathrm{HI}+\pi^{+}$. The changes made to calculate the cross section and analyzing power for $p+d \rightarrow{ }^{3} \mathrm{He}+\pi^{0}$ will be outlined at the end of this section.

The first diagram (a) corresponds to pion production mediated by the exchange of one nucleon in the t-channel. The vertex function which enters in the calculation of this amplitude is the overlap Ndt between one nucleon distributions in the triton and the deuteron. This amplitude is calculated for the deuteron and triton wave functions in momentum space.(the symbol $\mathrm{N}$ designates a nucleon). This diagram contributes mainly at forward angles.

The second diagram (b) corresponds to a two nucleon mechanism for pion 


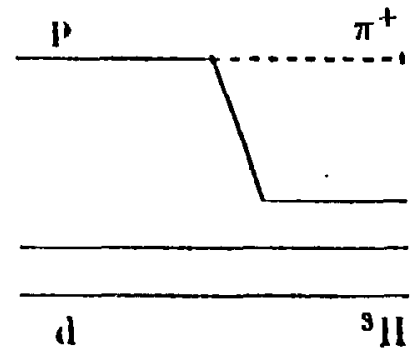

(a)

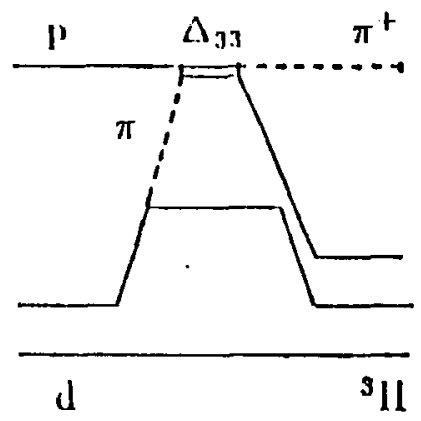

(c)

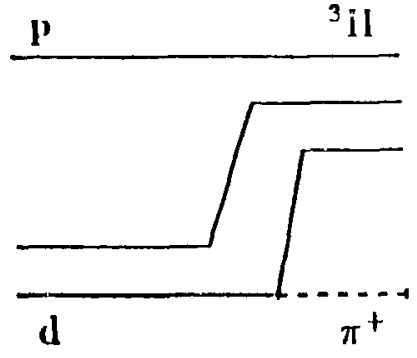

(b)

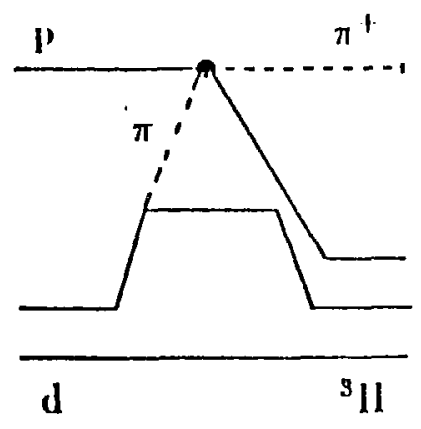

(d)

Figure 4.5: Diagrams fcr the amplitudes associated with the reaction $p+d \rightarrow{ }^{3} \mathrm{I}+\pi^{+}$ according to Laget and LeColley (Ref. 94). 
production, by interaction with the deuteron cluster. In the calculation of this diagram the vertex function involved are $d N N \pi$ and $t(N N) N$. In this amplitude the active pair in the triton is constrained to be in an isospin state $\mathrm{T}=1 \mathrm{in}$ order to conserve isospin at the vertex involving the deuteron. This diagram contributes both at forward and back angles with a minimum at $90{ }^{\circ}$.

The third diagram $(\mathrm{c})$ represents the formation for $\Delta_{33}$ resonance in the schanrel. The amplitude for this process involves the combination of elementary matrix elements $\sqrt{2} T_{\pi^{+} p \rightarrow \pi^{+} p}\left(\Delta^{++}\right.$intermediate state) and $-T_{\pi^{+} n_{n \rightarrow \pi^{0} p}}\left(\Delta^{+}\right.$intermediate state). In this diagram the active pair in the triton can be either in the $\mathrm{T} 0$ or in the $\mathrm{T}=1$ isospin state. Both the contributions have been calculated, but the process turns out to be dominated by the $\Delta$ formation in the $\mathrm{T}=0$ (pn) pair.

The last diagram (d) corresponds to non-resonant contribution to pion rescattering. The S,P,D partial waves are taken into account. They are calculated according to the phase shift fit by Rowe et al..$^{99}$.

The triton wavefunction used in the calculation of the vertex functions discussed above has been obtained ${ }^{100}$ by solving the Faddeev equations for the Reid soft-core potential. ${ }^{101}$ This potential is also used in the Schrödinger equation which gives the deuteron wavefunction.

In the calculations performed for the ninal state ${ }^{3} \mathrm{IIe}+\pi^{0}$, the mass effects associated with the mesons and baryons involved in the intermediate and final states are included. The correct values of the masses of the $\pi^{+}$and $\pi^{0}$ have been used, as well as the correct masses for ${ }^{3} \mathrm{iI}$ and ${ }^{3} \mathrm{He}$. In the diagrams involving the formation of the $\Delta$ resonance, the $\Delta^{++}$and $\Delta^{+}$intermediate states are involved in the case of ${ }^{3} \mathrm{H}$ final state, whereas $\Delta^{+}$and $\Delta^{0}$ are involved in the case of ${ }^{3}$ Ile. The values of the isobar masses adopted in the present $c^{-}$'rulation are respectively $M_{\Delta}=M_{\Delta^{++}} \approx M_{\Delta^{+}}=1230 \mathrm{MeV}$ for the ${ }^{3}$ If case and $M_{\Delta}=\frac{1}{2}\left(M_{\Delta^{+}}+M_{\Delta^{0}}\right)=$

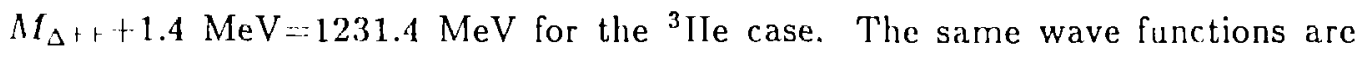
used for ${ }^{3} \mathrm{II}$ and ${ }^{3} \mathrm{He}$, i.e., no Coulomb induced wave function differences have 


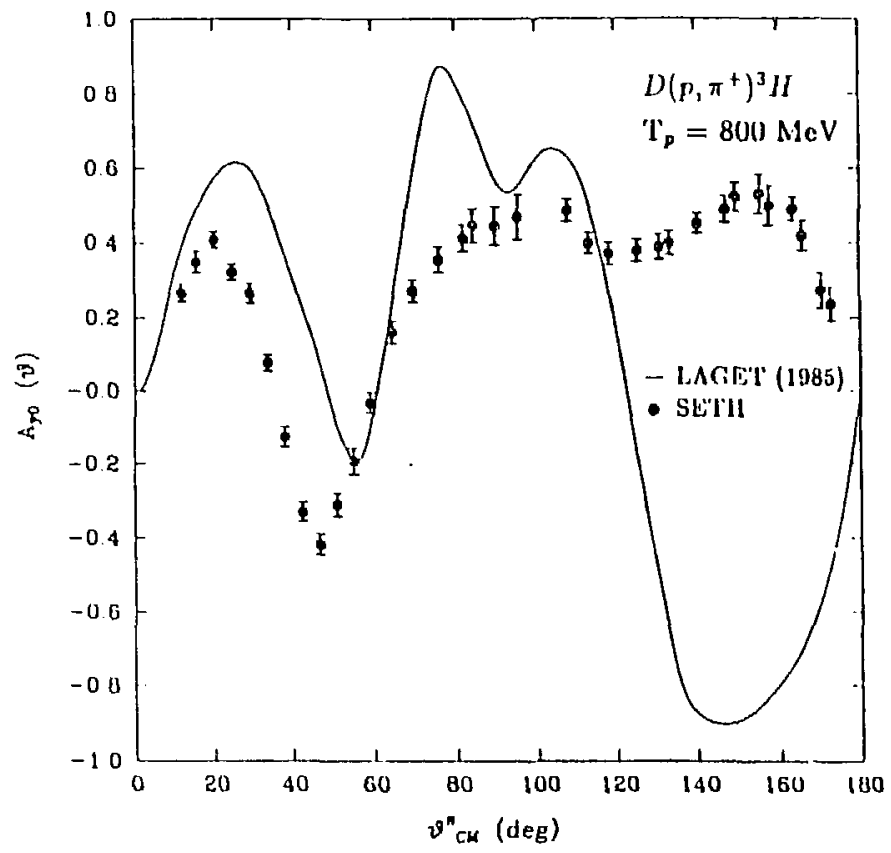

Figure 4.6: Analyzing power for the $p d \rightarrow \mathrm{t} \pi^{+}$reaction at $800 \mathrm{MeV}$. The experimental data are from Ref. 90 . The theoretical curve is from Ref. 98.

been considered.

Laget and LeColley have made $\sigma(\theta)$ and $A_{y 0}(\theta)$ calculations for us at several energies. Since complete data is available at $T_{p}=800 \mathrm{MeV}{ }^{90}$, we discuss the 800 $\mathrm{MeV}$ results first.

In Fig. 4.3 we show the results of these calculations for the differential cross sections at $800 \mathrm{MeV}$. We note that although the agreement of these calculations with the data is much better at forward angles than that obtained by Fearing, almost no improvement is observed at back angles. Fig. 4.6 shows the data and the predictions for $A_{y 0}(\theta)$ at $800 \mathrm{MeV}$. The features of the data at forward angles are 'qualitatively' reproduced, but at the back angles there is little similarity between the data and the predictions. Even the sign of $A_{\mu 0}(\theta)$ is not predicted correctly for $\theta \geq 120^{\circ}$.

In view of the above discouraging results for back-angle $\sigma(\theta)$ as well as $\Lambda_{v 0}(0)$, it would appear that little confidence can be had in the results for $R$ and $\Delta \Lambda_{y 0}(0)$ 
from this model. Ilowever, in absence of anything better, this is what we do below.

In Figs. 4.7 and 4.8 we show $\mathrm{R}$ and $100 \Delta A_{y^{0}} / A_{y^{0}}$ as calculated by Laget and LeColley for $\mathrm{T}_{\boldsymbol{p}}=730 \mathrm{MeV}$. From these calculations we obtain, for $\theta=130^{\circ}$ :

$$
\begin{gathered}
R^{\prime}=1.895 \\
{\left[100 \cdot \Delta A_{\mathrm{y} 0} /\left.\left\langle A_{\mathrm{y} 0}\right\rangle\right|_{t h}=-0.308\right.}
\end{gathered}
$$

As noted earlier, since Laget and LeColley did not include the external Coulomb corrections, we need to raise the ratio $\mathrm{R}^{\prime}$ by the external correction factor $=1.064$ as determined in Sec. 4.1. This leads to

$$
R_{t h}=1.064 R^{\prime}=? .014
$$

These 'theoretical predictions' (Eqns. 4.9 and 4.8) should be compared to our experimental results

$$
\begin{gathered}
R=2.193 \pm 0.007 \pm(0.024) \\
100 \cdot \Delta A_{y 0} /\left\langle A_{y 0}\right\rangle=-1.65 \pm 1.10 \pm(0.46)
\end{gathered}
$$

We note that our result for $R$ is significantly larger than the theoretical prediction. Perhaps this discrepancy indicates nothing more than the uncertainty in the theoretical prediction. We have already dwelt at length on the problems of the theoretical calculations. On the other hand, it is possible that the discrepancy is truly indicative of a charge independence breaking effect. Only better theoretical calculations can resolve this tantalizing question.

Our result for the difference in analyzing powers has the same sign as the theoretical prediction, but it is larger. However, the difference is not statistically too significant. A factor two to three improvement in experimental errors would be of grcat help. 


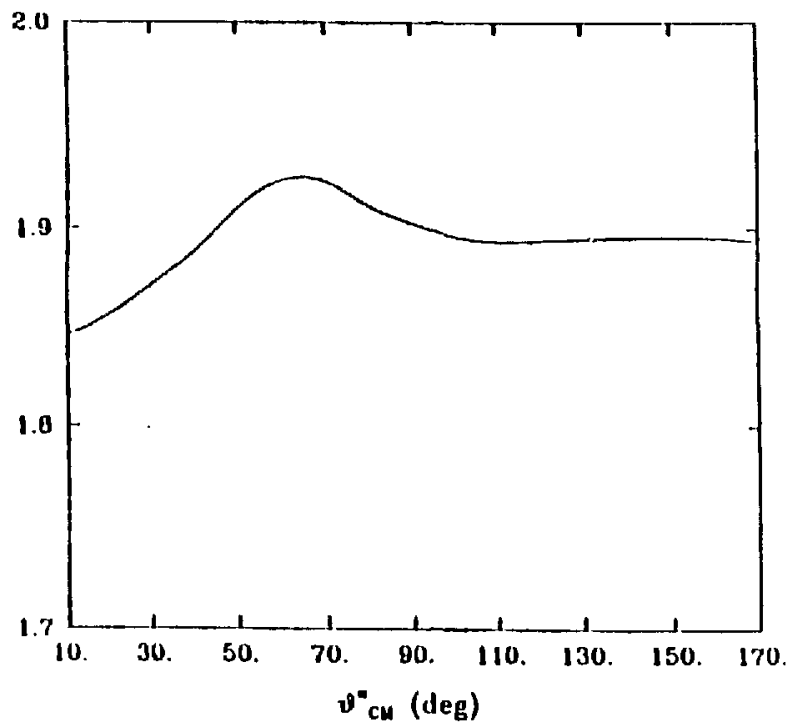

Figure 4.7: Laget and LeColley's prediction for the ratio $\sigma\left({ }^{3} \mathrm{II}+\pi^{+}\right) / \sigma\left({ }^{3} \mathrm{IIe}+\pi^{0}\right)$ at $T_{p}=730 \mathrm{MeV}$. These predictions include no corrections for external Coulomb effects.

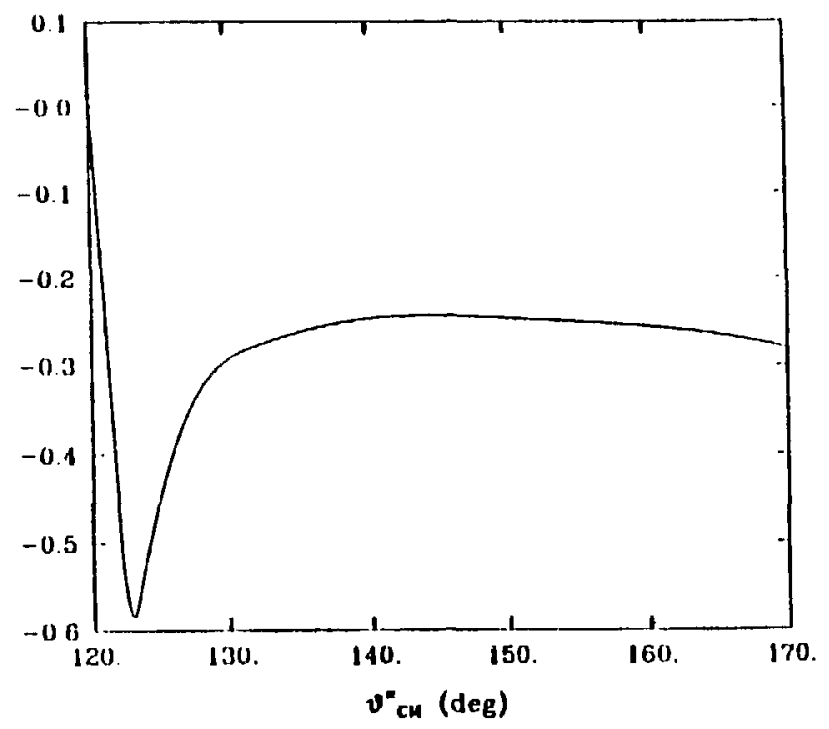

Figure 4.8: Laget and LeColley's prediction for the difference in analyzing power between $\vec{p}+d \rightarrow{ }^{3} \mathrm{II}+\pi^{+}$and $\vec{p}+d \rightarrow{ }^{3} \mathrm{He}+\pi^{0}$ reactions at $\mathrm{T}_{p}=730 \mathrm{MeV}$. 


\subsection{Conclusions.}

'The goal of this experiment was to make high precision measurements of the ratio, $\mathrm{R}$ between the differential cross sections and the diflerence $\Delta A_{y 0}$ between analyzing powers for the two reactions $\vec{p} d \rightarrow^{3} H \pi^{+}$and $\vec{p} d \rightarrow^{3} H e \pi^{0}$. We believe that we have satisfactorily realized this goal. Our new result for $R$ is almost an order of magnitude more precise than previous measurements, and we have made the first measurement of $\Delta A_{y 0}$ ever, with errors which are less than \pm 0.005 , or $\approx 1 \%$.

The theoretical interpretation of our results largely cludes us, at least so far. It is true that no theoretical explanations of charge symmetry or charge independence tests ever calculate the absolute values of the observables. Only the differences [e.g., scattering length differences $\Delta \mathrm{a}=\mathrm{a}_{n n}-\mathrm{a}_{n p}$, or analyzing power differences $\Delta A=A(\vec{n} p)-A(n \vec{p})]$, or ratios $[$ e.g., for the $p n \rightarrow d \pi$ reaction $\sigma(\theta \leq$ $\left.\left.90^{\circ}\right) / \sigma\left(\theta \geq 90^{\circ}\right)\right]$ are calculated. Ilowever, in most cases the general theoretical model for the observable is reasonably well understood and accepted. Unfortunately this is not the case with our reactions. As we liave shown, no satisfactory models for single pion production in pd collisions exist so far. The theory is beset with serious problems and there are indications (from the perennial difficulties of all the models at back angles) that some major piece of essential physics is missing in all the models extent. It is possible that despite the great progress made in few-nucleon physics in the last decade, the high moinentum components in these systems are still not well understood ${ }^{102}$. It is possible that $\Delta$-components in the two- and three-body systems play important role and need to be investigated seriously. Perhaps there are serious flaws in the use of impulse approximation. We are obviously not equipped to answer these questions. All we can hope is that our precision measurements will induce the theorists to pay serious attention to this important problem in few-body physics, in which study of ground state properties has so far been the preoccupation. 
In absence of reliable theoretical predictions we can not draw any hard conclusions from our results. The large value of $R$ observed can not be really interpreted unless at least the effect produced by Coulomb induced wave function differences is calculated with the best wave functions available today. We hope that this will happen soon. These wave function differences, in any case, should not affect analyzing powers, which are ratios of cross sections. Similarly many other uncertainties, e.g., distortions, should largely cancel out in $A_{y 0}$. This makes us bold enough to suggest that the $-1.6 \% \pm 1 \%$ difference in analyzing powers which we have measured does, in itself, indicate that charge independence breaking is at the level less than $\approx 2 \%$. This is a significant result because no measurements of charge independence breaking in this energy and momentum-transfer range have been made ever before. 


\section{References.}

[1] H. Weyl, Symmetry, Princeton Un. Press (1952).

[2] E. Noether, Nachr. Kgl. Ges. Wiss., Göttingen (1938) 235.

[3] C. N. Yang, R. L. Mills, Phys. Rev. $\underline{96}$ (1954) 197.

[4] S. J. Gates, et al., SUPERSPACE or One thousand and One Lessons in Supersymmetry, Benjamin/Cummings (1983) p.1.

[5] W. Heisenberg, Z. Phys. $\underline{77}$ (1932) 1.

[6] E. Wigner, Phys. Rev. 51(1937) 106.

[7] C. M. G. Lattes, H. Muirhead, C. F. Powell, and G. P. Occhialini, Nature $\underline{159}$ (1947) 694.

[8] H. Yukawa, Proc. Phys. Math. Soc. Japan 17, (1935) 48.

[9] N. Kemmer, Proc. Cambridge Phil. Soc. $\underline{34}$, (1938) 354.

[10] E. M. Henley in Isospin in nuclear Physics, ed. D. Wilkinson, (North Holland, Amsterdam 1969) Ch. 2.

[11] E. M. Henley, G. A. Miller in Mesons in nuclei, eds. M. Rho D. Wilkinson, (North Holland, Amsterdam 1979) vol. I, p. 405.

[12] E. M. Henley,in Proc. Nuci. Theory Summer Workshop, Sante Barbara, (1981) ed. G. F. Bertsch, (World Scientific, Singapore 1981) Ch. 1.

[13] S. A. Coon, M. D. Scadron Phys. Rev. $\underline{\text { C26 }}$ (198\%) 2402.

[14] E. M. Henley, T. E. Keliher, Nucl. Phys. A189 (1972) 63\%.

[15] K. Okamoto, C. Pask, Ann. Phys. (N. Y.) 68 (1971) 18.

[16] Stephenson, Gibson, in Proc. Int. Conf. on Nucl. Fhys., ed. J. deBoer, H. J. Mang (1973) p. 4.

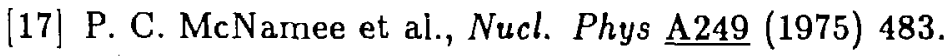

[18] L. Ge, J. P. Sv ne, Phys. Rev. C33 (1986) ¿17. 
(19) H. Baier, W. Bentz, Ch. Hajduk and P. U. Sauer: Nucl. Phys. A386 (1982) 460.

[20] H. Georgi, Weak interactions and modern particle theory, Benjamn Cummings (1984) 80.

[21] P. Langacker, H. Pagels, Phys. Rev. D19 (1979) 2070.

[22] P. Langacker, Phys. Rev. D20 (1979) 2983.

[23] L. Amethler et al.,Un. of Barcelona Preprint UAB-FT-102 (1984).

[24] F. Binon et al.,CERN Prepr. CERN-EP/8\&-27 (1984).

[25] D. B. Kapian, A. V. Manohar, Phys. Rev. Lett. $\underline{56}$ (1986) 2004.

[26| A. W. Thomas et al., Phys. Rev. D24 (1981) 2539.

[27] P. Langacker, D. A. Sparrow Phys. Rev. C25 (1982) 1194.

[28] R. P. Bickerstaff, A. W. Thomas, Phys. Rev. D25 (1982) 1869.

[29] S. Godfrey and N. Isgur, University of Toronto Preprint TRI-PP-85-110 (Dec. 1985).

[30] E. P. Wigner, Proc. of the Robert A. Welch Conf. on Chsm. Res. (Robert A. Welch Foundation, Houston, TX. 1957) Vol 1, p. 67.

[31] W. Benenson and E. Kashy, Revs. of Mod. Phys. 51 (1979) 523.

[32] J. A. Nolan, Jr. and J. P. Schiffer, Ann. Rev. Nuc. Sc. 19 (1969) 471.

[33] S. Schlomo, Rep. Prog. Phys. $\underline{41}$ (1978) 66

[34] E. G. Adelberger in Symmetries in Nucl. Structure, eds. K. Abrahams, K. Allart and A. E. L. Dieperink, (Plenum Press, N.Y.), 1982, p. 55.

[35] W. T. H. van Oers, Comments Nucl. Part. Phys. 10 (1982) 251.

[36] H. P. Noyes, Ann. Rev. Nucl. Sc. 22 (1972) 465.

[37] D. J. Knecht, P. F. Dahl, S. Messelt, Phys. Rev. 148 (1966) 1031.

[38] J. E. Brolley, J. D. Seagrave and J. G. Berry, Phys. Rev. 135B (1964) 119.

[39] M. M. Nagels et al., Nucl. Phys.B147 (1979) 189.

[40] P. U. Sauer and H. Walliser, J. Phys. G3 (1977) 1513.

[41] J. M. Allen and H. Fiedelday, Nucl. Phys. A260 (1976) 213.

[42] M. Rahman and G. A. Miller, Phys. Rev. C27 (1983) 917. 
[43| B. Gibioud et. al., Phys. Rev. Lett. $\underline{42}$ (1979) 1508, also Phys. Lett. 103B (1981) 9.

(44) D. W. Glasgow et al. Proc. Int. Conf. on Nucl. Data for basic and applied Science, Santa Fe (1985), to be published in Radiation Elfects; also D. W. Glasgow, priv. comm.

[45] E. Pedroni et al,,Nucl. Phys. A300 (1978) 321.

[46] B. Balestri et al., Nucl. Phys. A392 (1983) 217.

[47] T. G. Masterson et al., Phys. Rev. Lett. $\underline{47}$ (1981) 220, also Phys. Rev. $\underline{\text { C26 }}$ (1982) 2091.

[48] T. G. Masterson et al., Phys. Rev. C30 (1984) 2010.

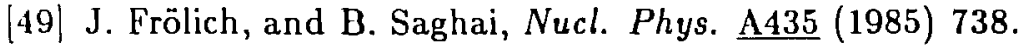

[50| B. M. K. Nefkens et al., Phys. Rev. Lett. $\underline{52}$ (1984j 735.

[51] Y. E. Kim, Phys. Rev. Lett. $\underline{53}$ (1984) 1508.

[52] S. Barshay, Phys. Rev. C311 (1985) 2133.

[53] C. Pillai et al., Bull. Amer. Phys. Soc. 31 (1986) 800, abst. DH2.

[54] M. Th. Kankhasayev et al. , Phys. Lett. (submitted), Dubna preprint, E4-85-612 (1985).

[55] C. L. Morris, et al., (unpublished).

[56] The Saturne Collaboration (unpublished), as reported by A. Stetz, Bull. Amer. Phys. Soc. 31 (1986) 848, abst. Hb4.

[57| Yu. K. Akimov, O.V. Savchenko and L.M. Soroko, Sov. Phys. JETP 14 (1962) 512.

[58] C. Y. Cheung, Phys. Lett. 119B (1982) 47.

[59] S. A. Coon,B. M. Preedom, Phys. Rev. C33 (1986) 605.

[60] C. L. Hollas, et al., Phys. Rev. C24 (1981) 1561.

[61] C. Y. Cheung, E. M. Henley and G. A. Miller, Phys. Rev. Lett. $\underline{43}$ (1979) 1215.

[62] L. Wolfenstein, Ann. Rev. Nucl. Sci. $\underline{6}$ (1956) 43.

[63] R. Abegg et al., Phys. Rev. Lett. 56 (1986) 2571; Proc. 6th Internat. Symp. on Polarization Phenomena in Nucl. Phys., Osaka, (1985), J. Phys. Soc. Japan, 55 (1986) 369. 
[64] G. A. Miller, A. W. Thomas, A. G. Williams, Phys. Rev. Lett. 56 (1986) 2567.

[65] S. E. Vigdor et al., Proc. Vth Internat. Symp. on Polarization Phenomena in Nucl. Phys. (Santa Fe, 1930), AIP Conf. Proc. 69 (1981) 1455; also L. D. Knutson et al., IUCF Scientific and Technical Report (1985) 12.

[66] C. Y. Cheung and R. Machleidt, contributed paper PANIC X, Heidelberg (1984) C13.

[67] M. Ruderman, Phys. Rev. $\underline{87}$ (1952) 383.

[68] E. Aslanides et al., Phys. Rev. Lett. 39 (1977) 1654.

[69] J. W. Low et al., Phys. Rev. C23 (1981) 1656.

[70] J. Вanaigs et al., Phys, Leit. 45B (1973) 1394.

[71] J. Källne et al., Phys. Rev. Lett. 40 (1978) 378.

[72] J.Franz et al., Phys. Lett. 93B (1980) 384.

[73] D.Kielczewska et al., Contributed Paper Karlsruhe (1983).

[74] K. C. Bandtel et al., Phys. Rev. 106 (1957) 802.

[75] A. V. Crewe et al., Phys. Rev. Lett. $\underline{2}$ (1959) 269.

[76] A. V. Crewe et al., Phys. Rev. 118 (1960) 1091.

[77] D. Harting et al., Phys. Rev. Lett. $\underline{3}$ (1959) 52.

[78] D. Harting et al., Phys. Rev. 119 (1960) 1716.

[79] N. E. Booth, Pinys. Rev. 132 (1963) 2305.

[80] B. H. Silverman et al.,Nucl. Phys. A44 (1985) 621.

[81] W. Dutty et al.,Proc 9th ICOHEPANS (1981) 184.

[82] M. V'. McNaughton, Phys. Rev. C23 (1981) 1128 and ref. therein.

[83] M. W. McNaughton, LAMPF Beam Line Polarimeters LA-8307-MS (1980).

[84] J. Novak, Private communication.

[85] B. Zeidman, The HRS Spectrometer I. Concepts and design. LA-4773-MS (1971).

[86] C. L. Morris et al., IEEE Trans. Nucl. Sci. 1 (1978). 
[87] C. L. Morris, NIM 196 (1982) 263.

[88] LAMPF Doc. MP1-1-3\$1\$-2 (1983) and references therein.

[89] K. Jones Notes on using RSX-11M at HRS (1984).

[90] Kamal K. Seth, to be published.

[91] H. S. Köhler, Phys. Rev. 118 (1969) 1345.

[92] S. A. Bludman, Phys. Rev. $\underline{94}$, (1954) 1722.

[93] J. L. Friar and B. F. Gibson, Ann. Rev. Nucl. Sci. $\underline{34}$ (1984) 403.

[94] H. W. Fearing, Phys. Rev. C11 (1975) 1210; C11 (1975) 1493; and $\mathrm{C} 16$ (1977) 313. Also H. W. Fearing, Progr. in Particle and Nucl. Phys. (1981).

[95] A. M. Green et al., Nucl. Phys. A316 (1979) 215; A329 (1979) 477.

[96] A. M. Green and J. A. Niskanen, Nucl. Phys. A271 (1976) 503; also J. Niskanen, Nucl. Phys. A298 (1978) 417.

[97] M. E. Sainio, Nucl. Phys. A389 (1982) 573.

[98] J. M. Laget and J. F. Lecolley, contributed paper PANIC X, Heidelberg (1984) E21; J. M. Laget priv. comm.

[99] G. Rowe et al., Phys. Rev. C18 (1978) 584.

[100] C. N. Hadjuck et al., Nucl. Phys. A322 (1979) 325 and R. A. Branderburg Phys. Rev. C12 (1975) 1368.

[101] R. V. Reid, Ann. Phys. (N. Y.) $\underline{59}(1968) 411$.

[102] F. Khanna Proc. $2^{\text {nd }}$ Conf. on Intersection between Part. and Nucl. Phys., Lake Louise, Canada (1986) and private communication.

[103] G. S. Blanpied, Los Alamos Thesis LA-i 262-T (1978).

[104] E. K. J. Zavoiskii, Transl. Soviet Phys. JETP $\underline{5}$ (1957) 603.

[105] L. Madanski, G. E. Owen, Phys. Rev. Lett. 2 (1959) 209.

[106] W. E. Lamb, R. C. Retheriord, Phys. Rev. $\underline{79}$ (1950) 549.

[107] W. Haeberli, Ann. Rev. Nucl. Sci. 17 (1967) 373. 


\section{Appendix $\mathbb{A}$}

\section{The linear accelerator.}

An overall view or the Los Alamos Meson Physics Facility has been given in Fig. 2.1. The different experimental areas shown in this figure employ a beam which is accelerated in a two-staged linear accelerator. This LINAC is able to accelerate simultaneously particles from two different sources. It has three independent injectors. The first one produces protons $\left(\mathrm{H}^{+}\right)$, the second produces unpolarized negative ions $\left(\mathrm{H}^{-}\right)$, and the third produces polarized negative ions $\left(\mathrm{P}^{-}\right)$. Some details on the polarized proton source are given in the next appendix.

The first stage has an Alvarez drift tube structure operating at 201.25 MHz. It consists of acceleration regions and drift regions of of increasing length to match the increasing particle velocity. In the drift tubes quadrupole lenses are used for focusing. The particles are accelerated to $100 \mathrm{MeV}$ in this stage and are then fed to the main accelerator. The frequency of this section is $805 \mathrm{MHz}$. It is capable of producing a beam with final energy which can be varied step-wise between 100 and $800 \mathrm{MeV}$. The $\mathrm{H}^{+}$and $\mathrm{H}^{-}$(or $\mathrm{P}^{-}$) beams are separated magnetically at the switchyard shown in Fig. 2.1. The $\mathrm{H}^{+}$beam goes to the production targets of line $\boldsymbol{\Lambda}$, from which several meson beams are generated.

The $\mathrm{H}^{-}$beam travels along line $\mathrm{X}$ up to a section where some stripper foils transform part of this beam into a $\mathrm{H}^{+}$beam by stripping the electrons in the negative ions. The unstripped portion of the beam goes to line $\mathrm{B}$. The protons are magnetically separated to go to line $\mathrm{C}$, which is the beam line associated with the HRS spectrometer. A plane view of line $\mathrm{C}$ has been given in Fig. 2.2. This 
bearn line consists of a separation section, a dispersion section and a matching and twisting section.

In the separation section the beam is bent by three consecutive small angles equal to $1^{\circ}, 1^{\circ}$, and $4^{\circ}$ respectively. Subsequently the beam is deflected by two angles of approximately $57^{\circ}$ in the dispersion section. $U_{P}$ to this point the dispersion of the beam is along the horizontal plane. As the HRS spectrometer operates in VHV mode (vertical dispersion, horizontal scattering, vertical momentum analysis), it is necessary to rotate the dispersion plane of the beam before it reaches the interaction region. This purpose is achieved by a set of quadrupole magnets which constitute the 'twister section'. Finally another set of quadrupole magnets provides the 'dispersion matching' function needed for the operation of the spectrometer in energy loss mode. In other words, they allow a tuning of the beam on target to optimize the energy resolution of the spectrometer. The design concept of 'energy loss mode' for high resolution spectrometers will be discussed in Appendix 3. More details on line $\mathrm{C}$ can be found in Ref. 103. 


\section{Appendix $B$}

\section{The polarized proton source.}

The method employed to produce polarized protons was first proposed by Zavoiski ${ }^{104}$ and Madanski and Owen ${ }^{105}$. It takes advantage of the unique properties of the metastable $2 \mathrm{~s}_{1 / 2}$ level of the hydrogen atom.

Fig. B.1 shows the energies of the $2 s_{1 / 2}$ and $2 p_{1 / 2}$ levels as function of an axial magnetic field applied to them.

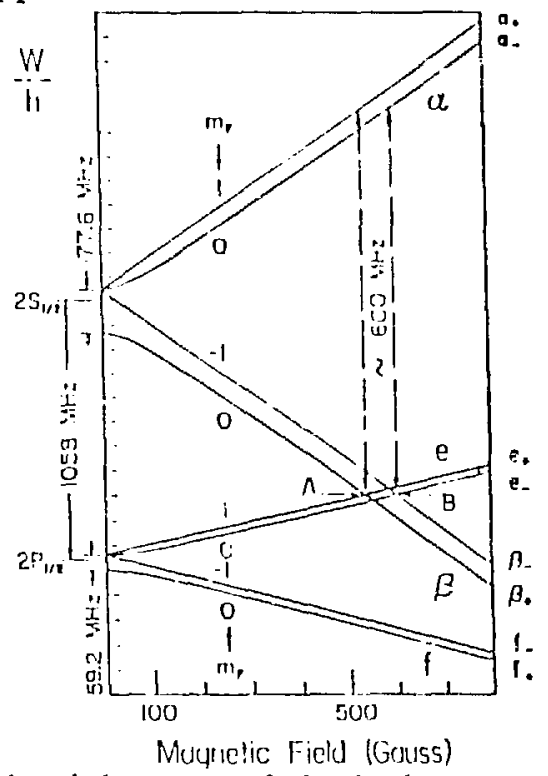

Figure B.1: Energy-level diagram of the hydrogen atom fur principal quantum number $n=2$ at an excitation energy of $10.2 \mathrm{eV}$. (from Ref. 107).

The splitting between the $2 \mathrm{~s}_{1 / 2}$ and $2 \mathrm{p}_{1 / 2}$ in absence of magnetic field is produced by Lamb shift and the degeneracy between levels having the same clectron spin 
projection is removed by the hyperfine interaction with the proton magnetic moment. Fig B.l shows clearly the principle on which this method is based. Atoms are pumped to the metastable state and a selective quenching of the levels $\alpha_{-}$and $\beta_{ \pm}$is performed. Under general conditions, a longitudinal electric field couples by the $\alpha$ and $e$ levels Stark mixing, whereas a transverse electric field couples the $\beta$ and $e$ levels. Besides, there is an interesting effect which was obseved for the first time by Lamb and Retheford. ${ }^{106}$ It affects the three lovels, $\alpha, \beta$, e, wherr a magnetic field $\mathrm{B}_{z} \approx 574 \mathrm{G}$ is applied (this field corresponds to the neighbourhood of the crossing point shown in Fig. B.1) together with a static tranverse electric field $\mathrm{E}_{y}$ and a radiofrequency longitudinal electric field $\mathrm{E}_{z}$. $\mathrm{E}_{y}$ couples the levels $\beta$ to the levels $e$ and $\mathrm{E}_{z}$ couples $\alpha$ to $e$. In absence of the levels $\beta, \alpha$ would have a broad dip in the intensity when the radiofrequency corresponds to its $z$, saration fiom the levels $e$ (which are rather broad). However, in the physical situation described above there are three sats of levels which are coupled, $\alpha, \beta$, and $e$. The presence of the level $\beta$ has the effect of 'sharpening' the quenching width, thus allowing sclective quenching of the $\alpha_{+}$or the $\alpha_{-}$levels depending upon the magnitude of $B_{z}$ (the two fields correspond to the points $A$ and $B$ of Fig. B.1.

The actual polarized source consists of three main sections.

In the first stage protons extracted from a duplasmatron are passed through a cloud of Cs atoms. Hydrogen atoms are formed through the charge exchange reaction:

$$
\mathrm{II}^{+}+\mathrm{Cs} \rightarrow \mathrm{H}_{2 s}+\mathrm{Cs}^{+} \text {. }
$$

Cs is chosen because it gives the most favourable ratio between the formation of hydrogen atoms in the $2 \mathrm{~s}$ state and in the ground state.

Next, there is a 'spin-filter' section, where a magnetic field $\mathrm{B}_{z}=540 \mathrm{G}$, a tranverse static electric field $F_{Y}=20 \mathrm{~V} / \mathrm{cm}$ and an axial $\mathrm{RF}$ field $\mathrm{E}_{z}=20 \mathrm{~V} / \mathrm{cmi}$ are applied.

Finally the hydrogen atoms are sent to an argon cell to produce $\mathrm{H}^{-}$ions. Again the choice of the gas is determined by the goal of selective ionization of 
atoms in the metastable state.

The negative hydrogen atoms (conventionally identified as $\mathrm{P}^{-}$) are at this point injected into the main accelerator. The polarization achieved by this method is fairly good (typically $\approx 0.8$ ). 


\section{Appendix $\mathrm{C}$}

\section{The HRS Spectrometer.}

The main design characteristics of the IIRS magnetic spectrometer can be summarized as follows ${ }^{85}$ :

- VHV (Vertical dispersion - Ilorizontal Scattering - Vertical Analysis) Energy loss configuration.

- Quadrupole-dipole-dipole arrangement.

- Mean radius of curvature $3.5 \mathrm{~m}$.

Solid angle acceptance 3.6 msr.

- Momentum resolution $2.44 \times 10^{-5}$

- Range of scattering angles $2.5^{\circ}-10^{\circ}$ with reduced beam intensity and $10^{\circ}-$ $170^{\circ}$ with full beam current available.

- Angular resolution $0.8 \mathrm{mr}$.

- Dispersion $\langle\delta \mathrm{x} \mid \delta \mathrm{p}\rangle 18.244 \mathrm{~cm} / \%$.

Fig. 2.3 shows the IIRS facility. The magnetic spectrometer is vacuum coupled to the scattering chamber through several fixed ports and bellows. The spectrometer consists of a quadrupole lens followed by two dipole magnets. The quadrupole focuses in the $\hat{x}$ direction (radial) and defocuses in the $\hat{y}$ direction. These optical characteristics were chosen in order to minimize the gap width. 
The spectrometer provides point to point focusing in the $\hat{x}$ (dispersion) direction and parallel to point focusing in the $\hat{y}$ (scattering) direction. Therefore, up to $1^{\text {st }}$ order terms in the spectrometer optics, the scattering angle and momentum analysis are decoupled.

In the dispersion direction the spectrometer is designed to operate in the 'energy loss mode'. The principle of this design approach is the consideration that excellent energy resolution is needed in nuclear physics experiments in order to identify the state of the residual undetected particle. If the cross section has a slow energy dependence and the cnergy spread of the beam is not too wide, it is only necessary to measure the momentum difference between the beam particle and the corresponding detected particle $\left(\mathrm{p}_{3}-\mathrm{p}_{1}\right)$. This purpose is achieved by focusing all the particles which undergo the same momentum loss at the same coordinate in the dispersion direction. The variation of the missing mass $M M$ corresponding to variations of the kinematical variables $p_{1}, p_{3}, \theta_{s c},\left(\theta_{s c}\right.$ is the scattering angle $)$ is given by:

$$
\Delta M M=\frac{\partial M M}{\partial p_{1}} \cdot \Delta p_{1}+\frac{\partial M M}{\partial p_{3}} \cdot \Delta p_{3}+\frac{\partial M M}{\partial \theta_{s c}} \cdot \Delta 0_{s c}
$$

The first two terms can be rewritten as:

$$
(\Delta M M)_{p}=\frac{\partial M M}{\partial p_{3}} \cdot p_{3}^{0} \cdot\left[\delta_{3}+\frac{p_{1}^{0}}{p_{3}^{0}} \frac{\partial M M / \partial p_{3}}{\partial M} \frac{\partial}{\partial / \partial p_{3}} \delta_{1}\right]
$$

where $\delta$ refers to the fractional deviation from central momentum, $p_{1}^{0}$ identifies the central momentum of the beam line, and $p_{3}^{0}$ identifies the central momentum of the spectrometer. The first order optics of the spectrometer gives:

$$
x_{f}=D_{s} \delta_{3}+R_{11} D_{b} \delta_{1}
$$

where $\mathrm{x}_{f}$ indicates the vertical coordinate at the spectrometer focal plane, $D_{b}$ represents the bearn dispersion, $D$, represents the spectrometer dispersion and $R_{11}$ corresponds to the vertical magnification factor of the spectrometer. Substituting $\delta_{3}$ from C.2 in C:.3, we obtain:

$$
(\Delta M M)_{p}=\frac{\partial M M}{\partial p_{3}} \cdot\left[\frac{x_{f}}{D_{s}}+R_{11} \frac{D_{b}}{D_{s}} \delta_{1}+\frac{p_{1}^{0}}{p_{3}^{0}} \frac{\partial M M / \partial p_{1}}{\partial p_{3}} \delta_{1}\right]
$$


'Therefore if:

$$
R_{11} \frac{D_{b}}{D_{3}}+\frac{p_{1}^{0}}{p_{3}^{0}} \cdot \frac{\partial M M / \partial p_{1}}{\partial M M / \partial p_{3}}=0
$$

the missing mass width is not affected, up to first order, by the momentum spread, $\partial p_{1}$ of the beam. Relationship C.5 defines the condition of 'dispersion matching' for optimum energy resolution. In other words, Eqrı. C.5 gives the dispersion $D_{b}$ to which the beam must be tuned in order that its energy spread does not affect the energy resolution of the spectrometer. 


\section{Appendix 0}

\section{Listing of the Monte Carlo simulation program.}

0
$\mathrm{c}$
0

c

c

c

0

c

o

c

Program $\|$ rsalm

paramater $\mathrm{J}-130$

jutamntine mn-nu

juramotar m-50

character"7 partlole, terget

character" 30 t1tie

byte $h(120)$

loglonl $\mathrm{kflag}, x \mathrm{flag}, \mathrm{f} f \mathrm{lg}, \mathrm{stopflag}$, test, coses

integer loption.

1 mopcion

Intoger segd, events, eventmax

tntagir nxl, nyl, nx2, ny2, laxng

integer awindow

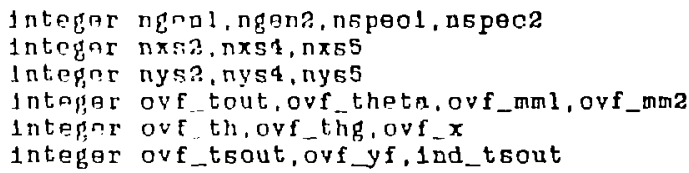


[at $\operatorname{no}(0)$

real angtgt(ma)

real the ripoo(ma)

ran 1 yfront $(m)$, ang, rog $(m), \operatorname{angmr}(m), \operatorname{ef} f(m)$

ronl minas $(j), x \in(j)$

real ekin(j), deltaspec( $J)$

common rpoil

common/elusso/olose, ec, stopflag

data thepec/la. I

chata the max, dthf/8u. , 2./

data clottno/49.5/

datio s.max/30./

data $y \max / 5.1$

data $\operatorname{ec}(4), \operatorname{\theta e}(5), \operatorname{ec}(7), \operatorname{ec}(8), \operatorname{ec}(9) / 2,1,20,7,2 * 0.1$

deta a thick . ga/

intan acint, nescht, polnt/3.65,6.23,62.6/

dinta o rnilds.8/

drta s thlok/. . B/

date $513 / 10,035,125,7,6 \mathrm{~L}, 1$

datn 5 र. $30,1,38,42.55 /$

data. $5 . . y / 5.35,6,5,7.2 /$

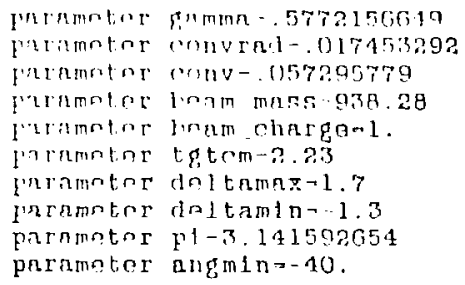

Real Input information about the case to be processed.

$\operatorname{Irnl}(1, \cdots, n+1-30)$ t $1 \mathrm{t} 1 \mathrm{\theta}$

rrail(1.) innmi, 10ptlon, aventmax, tbeam, tshift, ztarget

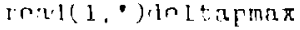

rotal $1, \cdot)$ particle, mrecoll, zrecoll, pcentral 
rnal(1, )target, mtarg, ztarg,atarg,titarg,t_rad, ma

ren'1 $(1, \cdot) m \cdot$, moption, mm $1 \mathrm{n}, \operatorname{mmax}, \mathrm{math}$

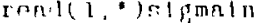

react $(1, \cdot)$ igmacof (1), s1gmacof (2), sigmacof (3)

rend ( 1, ) slght, sigtbt

reati $(1, \cdot)$ phitmin, phitmax

remil (1, *) thetatm Ln, thetatmax

read ( 1. ) thepleg thspar, thmin, thaax

rearl( 1.") Lowthl, h1 ghthl, lowth2, h1 ghthe

read 1 " ) he tapln, del tapout, ddel tapout

read ( 1 , ")mmassm $1 \mathrm{n}$, dmmass

Int talization step

$1 \times n \pi g \cdot 0$

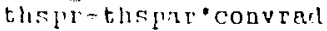

theprte-thepheg convrad

coctiun-corithrets)

ssatrur.sin $($ thisprtr)

athi-aln(thise)

cths +cos(thspr)

Banm angular ronrdinates in the HRS event analyzer are $(0,0,1)$ in thin lahintory frame and ( $0,-6$ ths, cths) in the spectrometer frame. (arģul kr divergonce supposed - 0)

xys. 0

Ylin stiss

zFa.ctha

\begin{tabular}{|c|}
\hline ind \\
\hline$n g(\cdot n \Omega-0$ \\
\hline$r$ Ferel 0 \\
\hline nspen?-0 \\
\hline $\begin{array}{l}k \text { ovf }-0 \\
n \vee f=-0\end{array}$ \\
\hline $\begin{array}{ll}n \vee f & x-0 \\
o \vee f & n \pi) \cdot 0\end{array}$ \\
\hline$O$ Y f \\
\hline $\begin{array}{l}0 \times f-\operatorname{th}-1 \\
\text { ove tout - }\end{array}$ \\
\hline $\begin{array}{l}\text { ove tnut-o } \\
\text { oyf thutn-o }\end{array}$ \\
\hline $\begin{array}{l}\text { nef thota-0 } \\
\text { ndetrotorl-o }\end{array}$ \\
\hline $\begin{array}{l}\text { ndetrector-0 } \\
\text { ncounter-0 }\end{array}$ \\
\hline $\begin{array}{l}n c 0 u n c e r-0 \\
n w 1 n \cdot 10 w=0\end{array}$ \\
\hline$n \times 1-0$ \\
\hline$n \times 2-0$ \\
\hline IIy $1-0$ \\
\hline nу2.01 \\
\hline$n \times 5 ?-1)$ \\
\hline $2: 1-0$ \\
\hline $\ln 55-0$ \\
\hline
\end{tabular}




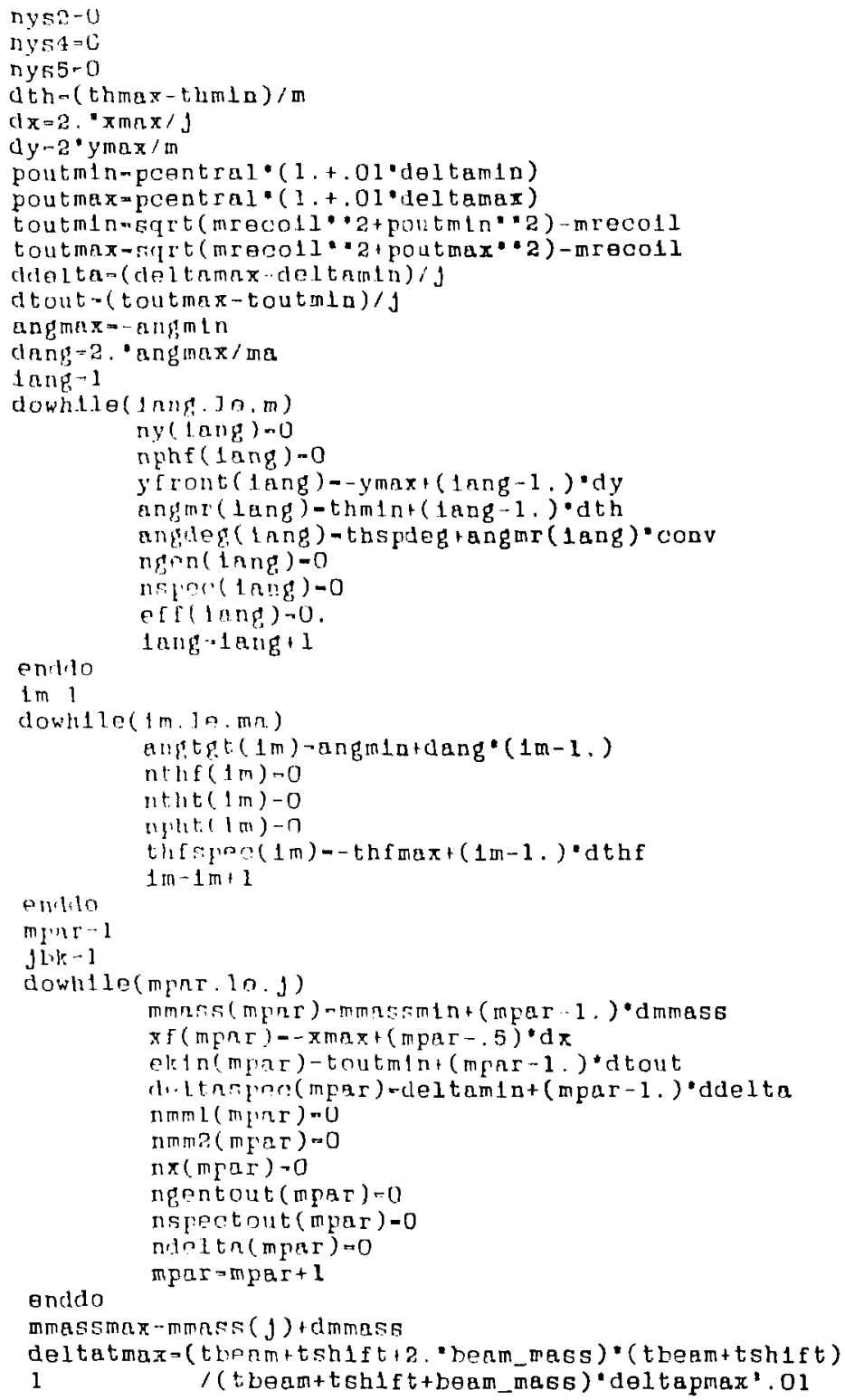

and $12-0.5 /$ in th 
$m \mu a r=m$

events $=1$

Generation of scattering events 1s now started.

pl-sqr't (tbeam" (tbeam+2. "beam_mass))-deltap1n

el $=5 q r t\left(p l * 2+b \theta a m \_m a s B * * 2\right)$

ekin beam-tbeam t tshift

dowhile (events. le. eventmax)

Each evenc 1 s characterized by (yt,phit, thetat, delta):

yt is generated according to gaussian distribution, delta by

simulation of energy loss at the target

(straggling approximeted by gauseian distribution

with sigma(E)).

$x \mathrm{flg} g-$ t tน

yf $l$ ag $=$. true.

if(1option.eq. 0$)$ then

: beam $r$ randon(0., ztarget)

elsg if (10ption.eq.1) then

zibenm-0.

els if (1option.eq.2) then

$z$ iveam $=z$ target

endef

yt--.61.2.t.gauss (dummy)

phito-rand om (ph1tmin, ph1tma $\pi$ )

thetato=random(thetatm1n, thetatmax)

c $\cdots$ - $n-r a n d o m(-d e i t a t m a x, d e l t a t m a x)$

is ( zheam.ne.0.) then

sigma-sigmain* Gqrt(zbeam)

tin stragl= 1 gma'gauss (dummy)

else

$\operatorname{tin} \operatorname{stg} 1=0$.

endif

tino-ekin_bean+deltatin

ec $(1)-\operatorname{tin} \bar{O}$

ec(2)-beam_mtiss

ec (3) -beam_uharge

$\theta c(6)=z$ beam

$\mathrm{ec}(1)=\mathrm{atarg}$

ec $(5)=z \operatorname{tar} g$

es(t)-titarg

cnll glosst

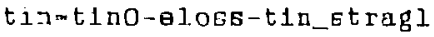


Multipla Coulomb scettering of beam up to the lateraction point 1 is now calculated.

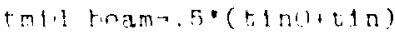

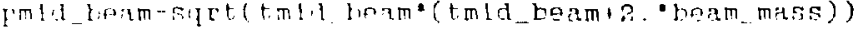

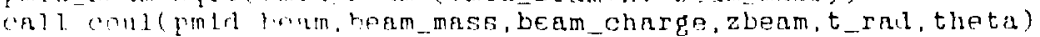

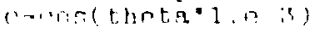

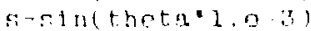

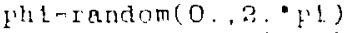

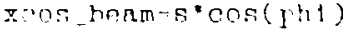

Yos horm-s*sin ( Fh1)

seis ham $\tan =0$

zoon $t-1 .-50 \cdot 6 \cdot($ thl. $20 \cdot 3+$ thetatoma)

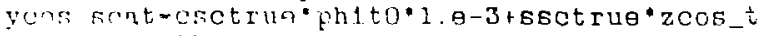

xine soat inthatato:l A-

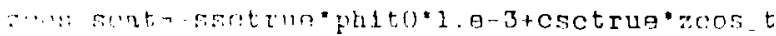

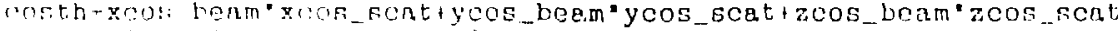

c thecat $=(\operatorname{tac}(\cos u)-\operatorname{thsprtr}) \cdot 1$. es

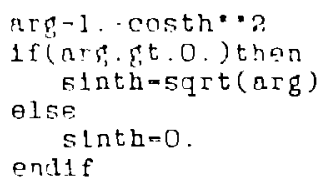

Avnargn enngsy lors of the beam particle 10 the thickness traversod by it 1a now criculated.

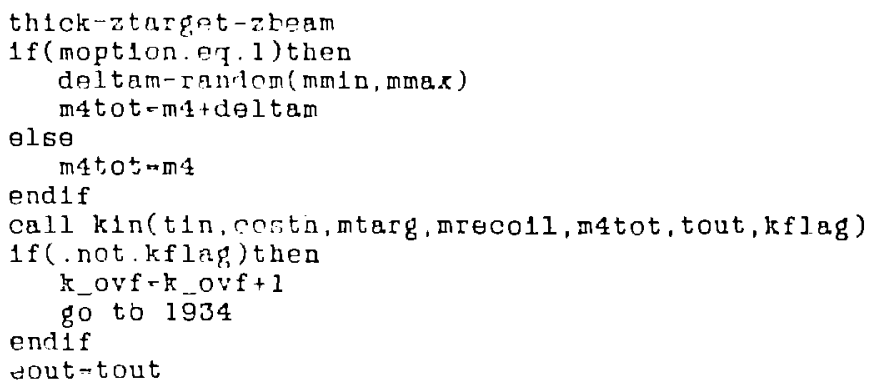

Average nnorgy loss of the detected narticle from interaction point to end of the target is now generatrid. 
C

c

c

c

c

c

$\mathrm{c}$

c

C

c

c

c

c

$\mathrm{c}$

c

c

C

ec( 1 )-pont

ec(a)-mracoll

$\operatorname{ec}(3)-2 \operatorname{recol} 1$

$\operatorname{ec}(6)-\operatorname{th} 1, k$

$\operatorname{\theta c}(\mathrm{t})-\sin \mathrm{s}$

$\operatorname{ec}(5)-7.5: 15$

$\operatorname{eo}(7)-t i t u r g$

call elcset

If(thlik. no.i, inom

1

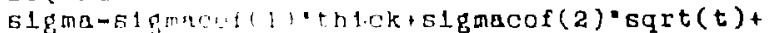

G.1

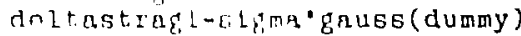

e) $1: 0$

nislif

doltastang $1-[1$.

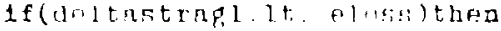

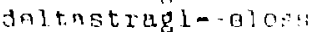

endif

deltue-eloestalelastrag,

tout - eout - deltae

if (tous.gt. o. ) then

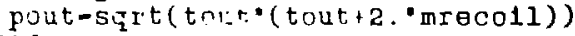

end $1 f$

$\operatorname{tm} 1 d-0.5 *($ eout + tout $)$

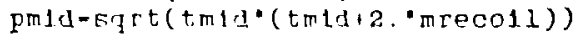

call crul ( $\mathrm{cmid}, \mathrm{mrecol1}, \mathrm{arec011}, \mathrm{thlck}, \mathrm{t}$ rad, tata)

$c=\cos (t h e t a \cdot 1, \theta-3)$

$\operatorname{sis} 1 \mathrm{n}($ theta*1.e-3)

phi-random(0.,2, p1)

phit-ph1to.s1 qpht 'gauss (dummy)

thetat-thetato+s1ptht "gauss(dummy)

$z_{-} \cos =1 .-59 \cdot 6^{\circ}($ phtt $\cdots+$ thetat $=2)$

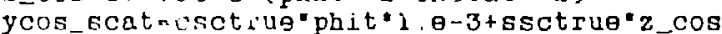

xcos Ecat $=$ thetat' $1 \cdot \theta-3$

zcos_scat--ssctrue ph1t"?.e-3+csctrue*_cos

call dev1(xcos_scat,ycos_scat,zcos_scat,c,ph1)

thetat...rcos_scat 1.93

ycos_sp-csctrue'ycos_scat-sectrue'zcos_scat

ph1t-ycos_sp'1. 13

yw1ndow-yt +50 . "ynns. $5 p$

call acceptance (ywlador, 5, 0, 5, xflag,yflag)

If ( not. $x$ f lag)then

nw1 ndow-nw1 ndow+1 
endif ${ }^{\text {go to }} 1934$

0

0

if ( not, yflag) yflag-.true,

call asslgn(m, lndthg, ovf_theta, thmax, thmin, dth, thsoat, ngen)

If (thscat. gt. highthl. or. thsoat. It. lowthl)then 80 to 1953

else $1 \mathrm{f}$ (thscat.gt.hightha.or.thsoat. It. lowtha) then go to 1933

endif

ngena-ngena+1

ngenl-ngenl+1

dot $=y p a * y p o r z p a * z p o$

if (dot.dt.0.9999999)dot-0. 9999999

thso-acos (dot)

ung ( thse-threr $) \cdot 1.03$

stheo=atn(thso)

othso =zoOA_aot

delta =(pout-peantral)/poentral*100.

xdel ta-gause (dummy)

If (xtelta.gt.0.) the

deltavar-6.510-3"2 slta

elso

deltavar $=9,12 \theta-3 * r d e l t a$

end $1 f$

deltandeltaideltavar

pout-poentral'(1.+.01'delta)

p3-pout+doltapout +ddel tapout delta

ej-6qrt $(p 3 \cdot a+m r \theta 0011 \cdot a)$

oall asalgn( $J$, ind_tout, ovf_tout, toutmax, toutmia,

I dtout, tout, agentout)

oall hre(xfl, thatofl,yfl, phifl,yt, phlt, thetat,delta)

Qall aoooptanoe ( $x \neq 1, x m n x, y f l, y \max , x f ! a g, y f l a g)$

if ( not. $x f l a g$. and. . not . yflag) then

$n \times 1-n \times 1+1$

ny $1=n y]+1$

go to 1934

endif

If ( not. $\pi$ flag)then

$\mathrm{n} \pi \mathrm{l}-\mathrm{n} \pi \mathrm{l}+\mathrm{l}$

go to $29: 34$ 
c

c

c else $1 f($ not.yflag)then

$$
\text { ny } 1 \rightarrow \text { ny } 1+1
$$

endif

$$
\text { go to } 1934
$$

$x \cos =$ thet $\mathrm{af} 1 \cdot 1 \cdot \mathrm{\theta}-3$

$y \cos -p h i f 1 \cdot 1 . \theta-3$

$z \cos =1 .-0.5 \cdot(x \cos * 2+y \cos \cdot 2)$

call transport ( $x f 1, x \cos , y f 1, y \cos$, deltaz, $x f 2, y f 2)$

call acceptance ( $x f 2, x \max , y f 2, y \max , x f l a g, y f l a g)$

if ( . nit . $x$ fleg. and. . not.yfleg ) then

$n \times 2-n \times 2+1$

ny $2-n y z+1$

go to 1934

ondif

If (. not. $x$ f $l \mathrm{gg})$ then

$\mathrm{n} \times 2=\mathrm{n} \times 2+1$

\&o to 1934

olso if (. not, yflog)then

ny $2-$ ny $2+1$

endif

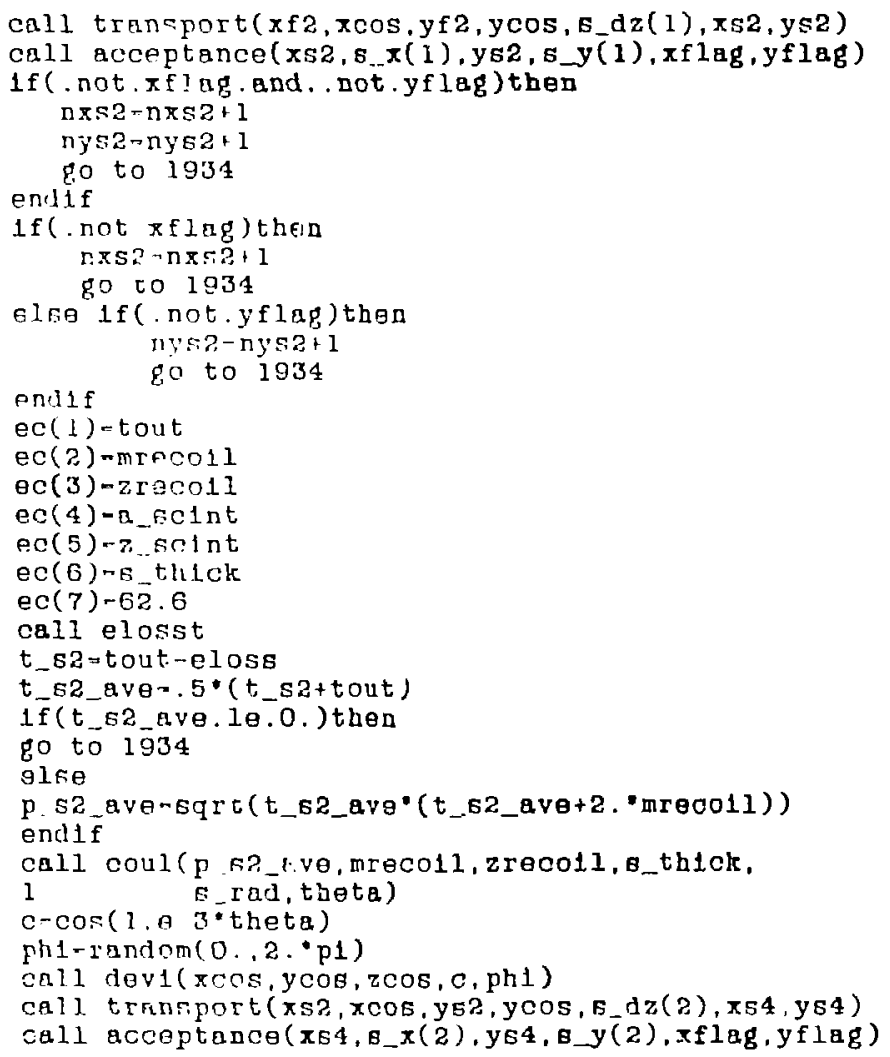


If ( not . $x f \log$. and. not yf lag) then

nxs4-nxs4+1

nys $4=$ nys $4+1$

ondif

go to 1934

If (. not. $x$ flag)then

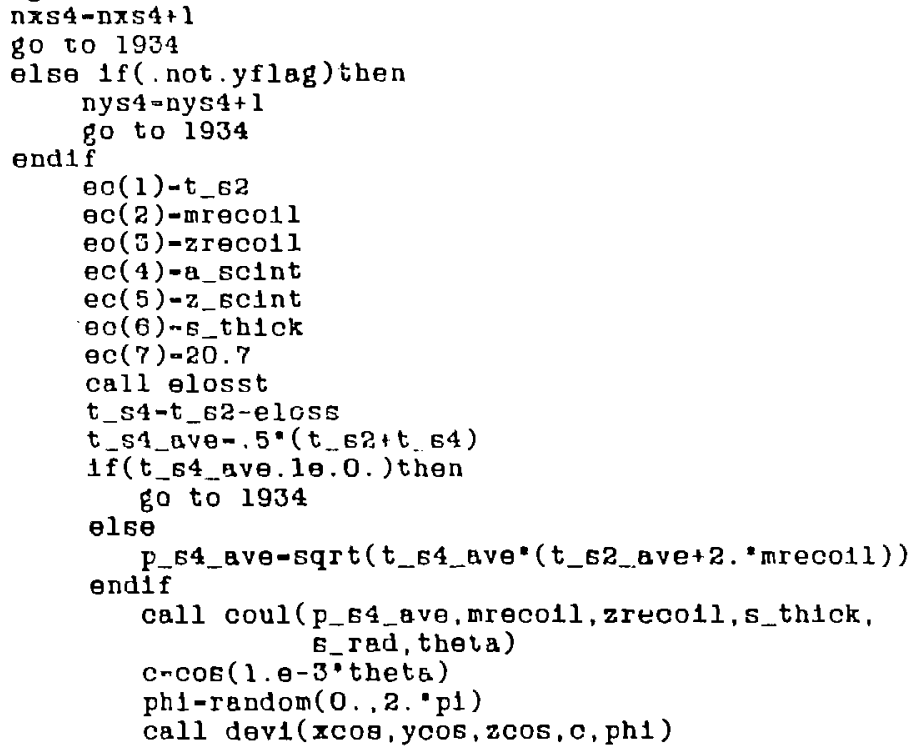

cn]l tranfport $(x, 4, \pi \cos , y 64, y \cos , 5 \mathrm{dz}(3), x \mathrm{~s}, \mathrm{ys} 5)$

call acceptance ( $\left.85,5 \_x(3), y s 5, s_{-} y(3), x f l a g, y f l a g\right)$

If ( . not. $x$ flag. and. . not. yflag) then

$n \times s 5=n \times s 5+1$

nys 5-nys $5+1$

go to 1934

end $1 f$

If (. not. $\pi f l a g)$ then

nxs5-nxs5+1

go to 1934

else if (. not, yflag) then

nys $5-$ nys $5+1$

end1f go to 1934

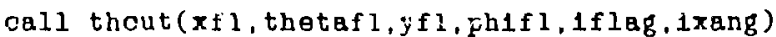

If $(1 \mathrm{flag} \cdot \theta \mathrm{g} .0)$ go to 1934

p4x-m-

p $4 y=-p 3 * 51$ nca

p $1 z-p 1-p 3 * \operatorname{cosca}$

$\mathrm{p} 4 \mathrm{sq}-\mathrm{p} 4 \mathrm{x} \cdot \cdot \mathrm{z}+\mathrm{p} \cdot \mathrm{y} \mathrm{y}^{*} \cdot \mathrm{z}+\mathrm{p} 4 \mathrm{z} \cdot * 2$

de-el-e3+ma-m4th 
1833

1853

2033

1934

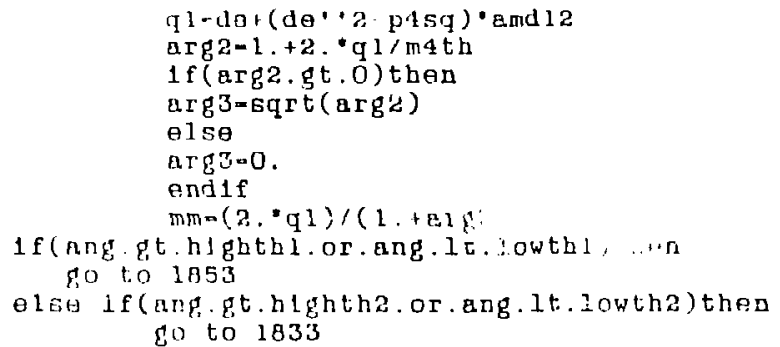


output information is written to f1le e795.uut

$1=1$

dowh1le(1.1e.120)

$b(1)-1 b-$

$i=1+1$

enddo

wr1te $(2,299)$ t 1 t le

write $(2,300) \mathrm{b}$

wr1te $(2,303) b$

write(2,301)eventmax,

1 tbeam,

1 tahift

1 deltatmax,

1 ztarget,

1 nartiole.

1 poentral

wr1te(a,700)targot

write(a,701)mtarg

write(a, 70a)m4

If (moption. $\theta$. 1) then

write $(2,703)$ mm $10, \operatorname{mmax}$

end $1 f$

wr1te(2,705) $\mathrm{m} 2, \mathrm{~m} 4 \mathrm{th}$

write $(2,605)$ thetatmin, thetatmax, o1gtht,phitmin, phicmax, 81gpht

write(2,706)thspdeg, thspar

write(2,707)del tap1n, del tapout, dde1 tapout

write( $2,30 a) b$, ncounter, ovf_theta, ndeteoted, ovf_th

write(a,304)r-f_delta, ovf_tsout,ove_tout

wr1to(3,502) nwindow, $2 \times 1, n y 1, n \times 2, n y a$

wrtte $(2,500) n \pi s 2$, dys2, nxs4, nys4, nxs8, nyss, 1xang

write(2,507)lowthl,highthl, nspool

write(a, 808) lowthl, h1 ghthl, oggnl

write $(2,509)$ lowthl, h1 ghthl, effl

wr1te(a,810) lowtha, h1 ghtha, nspeca

wr1te $(2,511)$ lowth2, h1ghth2, agena

write(2,812)lowtba,bightha, effa

Variables in the dispergion plang are now vilten to output flle.

write(a.603)b

wrlte $(2, B 04)(\pi f(k), n \pi(k), k-1, j)$

wrlte $(9,504)(x i(k), n x(k), k-1, j)$

write $(a, 603) b$

w: 1 te $(2,601)(\operatorname{thf}$ peo(k), $\operatorname{tin} f(k), k-1$, ma)

write $(10,601)(\operatorname{tbf}$ speo(k), athf(k),k-1,ma)

write $(2,802) b$ 


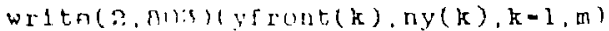

writa(?.Mrl h)

wr t.te(2.805)(angmr(k), $\operatorname{sph}(k), k-1, \pi)$

c

C

C

$c$

c

50

51

$\mathrm{c}$

$\mathrm{C}$

$\mathrm{c}$

write(a.rag)

wr1 te (2, g01)b

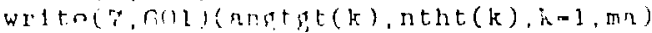

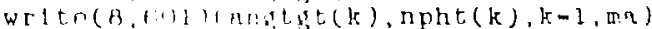

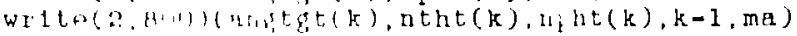

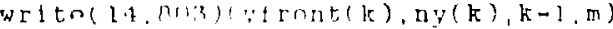

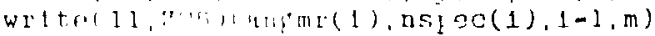

wrltols, $314 i)$

wrten(2:, : $: 2:: 1)$

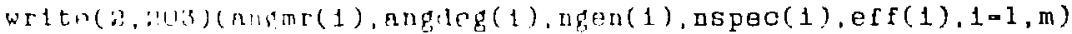

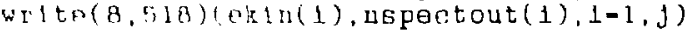

wr $1 \operatorname{ton}(2,5 \mid 1) !)$

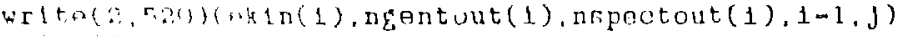

writin(2.101,1)

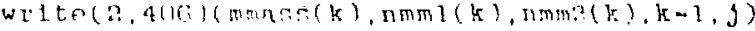

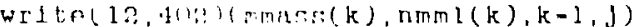

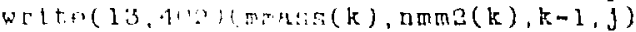

$\omega=1 \tan ? \cdot 41+1$

थ $11 \cdots ! 2,1+1 !$

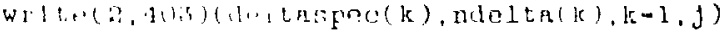

$\operatorname{cosen}(\operatorname{an} 1 t-14)$

close (mit-3)

close $(u n \perp t-r)$

close (undt-8)

close (undt.g)

closn (un1t-10)

clorn (un $1 t-11)$

clusen( $\ln 1 t-12)$

close (untt-13)

ondilo

ciscosa. falsa.

stop

folmat ( l', 'RE/MgV',k2, $(1 \pi, f t, 0) /)$

formet $(\cdot k 1,(1 \pi, f 6.2, \cdot k 2,(1 \pi, 14) /))$

format ( $m,(5 \pi, f 10.5,5 \pi, f 10.5,5 \pi, 110,5 \pi, 110,5 \pi, f 10.6 /))$

formet (' 1 ', $5 \pi, a 30)$

format $(5 x, 120 a 1 / 1 / / /)$

format ( $5 x$, 'llumber of events generated $\quad-', 2 x, 17 /$

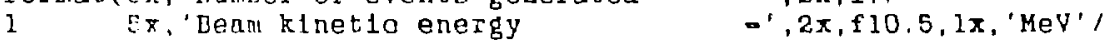

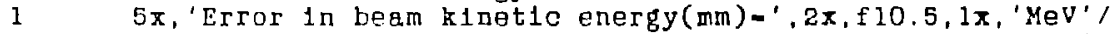

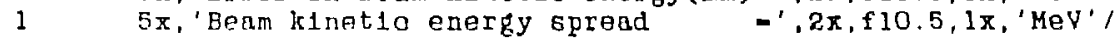

$15 x$, Target thickness

$15 x$, 'Farticle cietected format ( ' 1 ;

$5 x$.' Spectrometer certral momentum $\quad=$ '. $2 x, f 10.5,1 x$, 'Mev/c')

$15 x \cdot$ Output Information: $/ 5 x, 12091 / / 1$

$15 x$. 'number of events processed in selected theta region - , $2 x$ 
1 5x. 'number of overflows in scattering angle generated

$-.2 x$

$5 \pi$.'Total number of particles detected

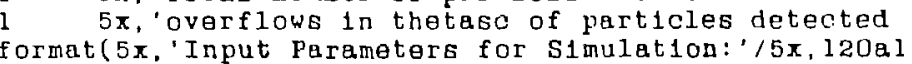

$=.5 \mathrm{x}$

$=2.2 x$

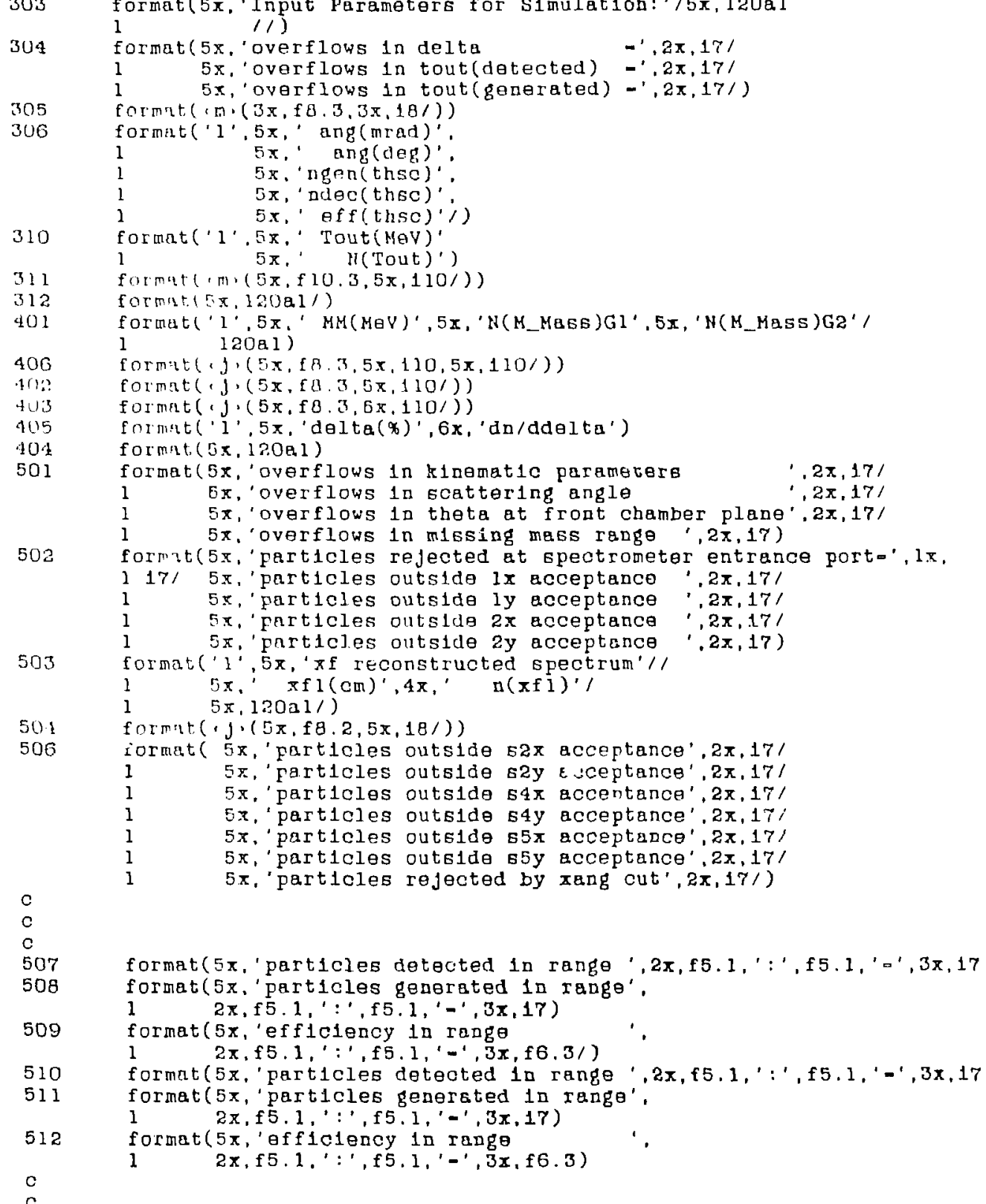



518

c

c

c

$c$

$\mathrm{c}$

$\mathrm{c}$ formut $(y,(5 x, f 10,3,5 x, 17 /))$

format (' 1 '.5x. 'Speotrum of k1net1o energy of detected particles'/// $1 \quad 5 x$,' Tout (MeV)',

$1 \quad 6 x$, ' Hgen(Tout)',

$15 x$.'Ndetected (tout)',

1 laval)

format $(\cdot \mathrm{J},(5 \mathrm{x}, \mathrm{f} 14.3,5 \mathrm{x}, 114,5 \mathrm{x}, 114 /))$

formst: $(+\operatorname{ma},(5 x, f 7.2,5 x, 17 /))$

format (' 1 ', $5 \pi, \operatorname{ang}(\operatorname{mr}) ', 6 \pi,{ }^{\prime} \mathrm{da} / \mathrm{dthf} /$ $15 \pi, 120 \mathrm{al})$

format $5 \pi$, 'm $1 \mathrm{n}$ th_nt_tgt (mr) -', $1 x, f 6,21$

$15 \pi, \max$ that tgt (mr) $\sim^{\prime} 1 \mathrm{k}, \mathrm{f} 6.21$

$15 x, 61 g m a$ th at tgt $(m r)-\cdots, 1 x, f 6.21$

$15 x$.'min $F^{h}$ at tgt $(m r)=1,1 x, f 6.21$

$15 x, m u x$ ph at tgt (mr) $=1,1 x, 16,2 /$

$15 \pi, \cdot 61 g m e$ ph_at tgt (mr) $\left.)^{\prime}, 1 x, f 6.2\right)$

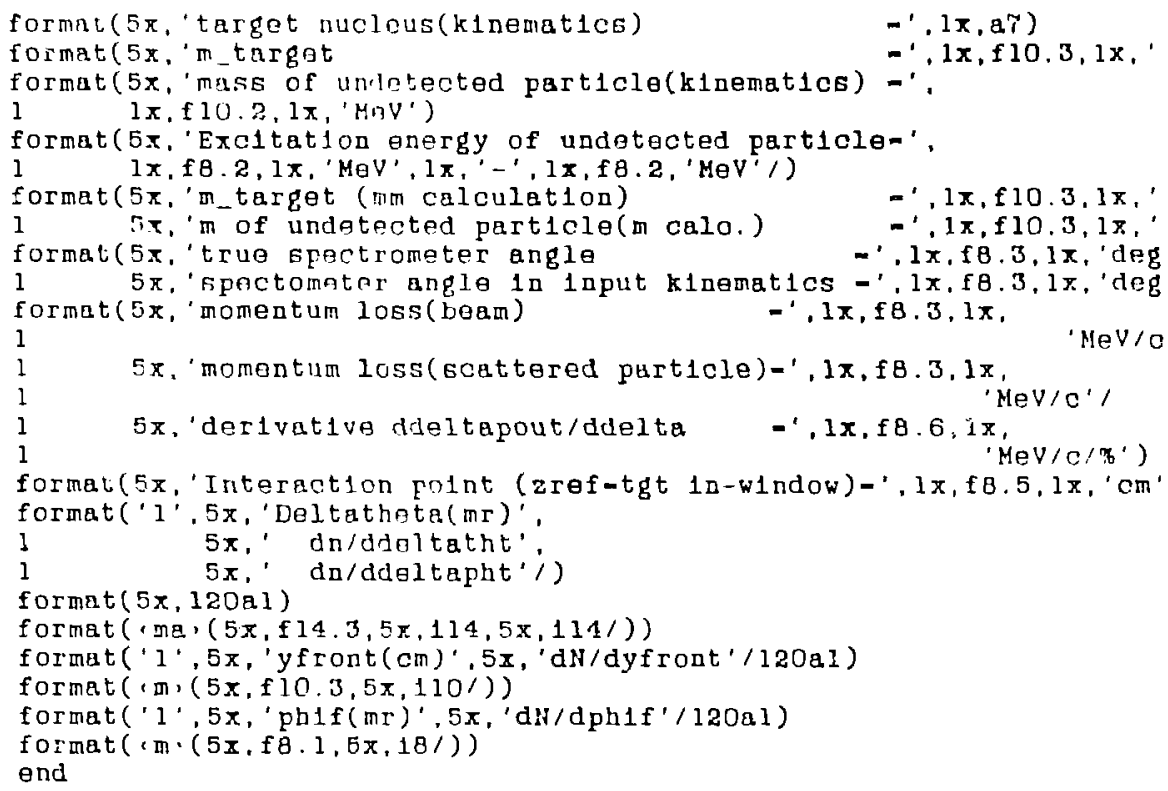


$10.1426 \theta-04^{\circ}$ delta ph1t $-0.783 \theta-05^{\circ}$ thetat phit

thttarl $=6.160+17.089^{*}$ de1ta-1.2219*thetat +

l $\quad-0.13567 * y \mathrm{t}-0.66019 \theta-02 * \mathrm{ph} 1 \mathrm{t}+$

$0.14756 \cdot$ delta*'2-0:079819*del ta"thetıt.

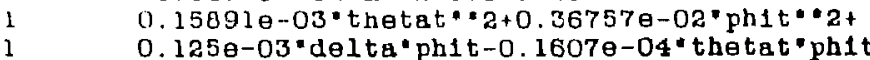

c

0

c

c

coce $0.429 \theta-1-0.261 \theta-2 \cdot \operatorname{If} 1$

d.1.l- $1619 \cdot .1032 \theta-2^{\circ} \mathrm{xf} 1$

กяก $=5.3439+0.0347^{*} \times f 1+0.0011^{*}$ thetef 1

llh- $0.09598+0.00939^{*} \times f l-0.0215$ thetef 1

det - rag "ddd-bbb'coo

if (det.er. O.) then

y $f l=100$.

o $15 \theta$ phif $1=1000$.

$y \operatorname{sh} 1 \mathrm{ft}=\mathrm{yt}-.0123+0.246 \theta-2 \cdot \operatorname{If} 1+0.139 \theta-2 \cdot$ thetaf 1

ph1shlft - ph1t $+2.01-0.0116^{\circ} \times \mathrm{f} 1+0.0027$ thetaf 1

yf $l=\left(p h 1 s h 1 f t{ }^{*} d d d-y s h 1 f t \cdot b b b\right) / d \theta t$

endif

phifl - (age*ysh1ft-cec*phish1ft)/det

roturn

end

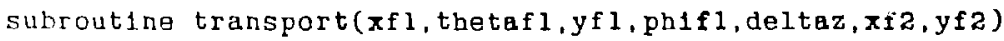

$x[2-\pi f l+d e l t a z$ thetaf 1

yfa-yfl+deltaz'phifl

return

end

subroutine acceptance( $x f, x m a x, y f, y m a x, f \operatorname{lag} x, f \operatorname{lag} y)$

$\log 1 \mathrm{cal} f \log x, f \operatorname{lag} y$

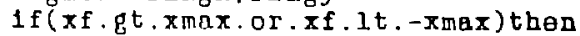

$f l g g x-$ false.

end $1 f$

If (yf.gt. ymnx. or.yf. lt. $-y m a x)$ then

indif flagy-. false.

return

end 
Subrout1ne thout( $x f l$, thetafl, yf 1, phlf 1,1 flag, 1xang)

c

$\mathrm{c}$

c.

parameter thoutlow $=-29.51$

parameter thouth1gh-29.29

c

c

$\mathrm{c}$

If $\log -1$

the $=-0,515521 \mathrm{E}+01+$

$1 \quad x f 1 * 0.672947 \mathrm{E}+00+$

1 thetafl-0.805446E+00+

1 yf $1 \cdot .351555 \mathrm{E}-01+$

1 phifl.0.129565E-01+

$1 \quad x f 1 \cdot 2 *-0.487944 E-02+$

1 xfl thetefl'0.319828E-02+

1 thetaf $1 \cdot 2 \cdot 0.513152 \mathrm{E}-04+$

1 yf $1 * 2 \cdot 0.489751 \mathrm{E}-02+$

1 yfl.ph1fi*-0.340292E-02+

l phifi*2*0.257161E-03+

$1 \quad x \neq 1 \cdot .26$

c

If (tho.1t. thout low. or tho.gt. theuthigh) then

If $\log _{\mathrm{g}}=0$

endif

1xang=1xang+1

return

end 
Subroutina Coul (pout,mrecoll,zrecoll, thick,lsad theta)

integer seed

renl liad

real mrecoll, Iratio

common seed

if (thick. a. o. ) then

that $a=0$.

raturn

$e_{i}$ ' $f$

liationthick/lind

pbeta-pout *2/sqrt (pout * $2+\operatorname{mrago11} \cdot a)$

irat $10-\operatorname{th} 1 \mathrm{ck} / \mathrm{lrad}$

s1gmatheta-14.1e+3/pbeta*zrecoll 6 grt (1rat10)*

I $(1 .+0.111111111111 \cdot \operatorname{alog} 10(1$ rat10) $)$

temp-gruss (dummy)

thetargigmatheta temp

return

and

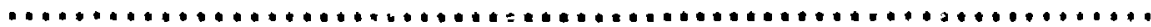

Thle flle colleote mlscelianeous short subroutines and functions called by the program SPECTRUM.

FUNCTION RANDOM (RMIN, RMAX)

Thls function generates a uniform distribution defined in an interval comprised between RMIN and RMAI.

INTEGER SEFD

COMMINI SEF.D

rarg-ran (seed)

rtemp-rorg*( RMAX-IMIN) +RMIN

rariclom $=$ rtemp

RE'TURY

END

FUHCTIUN GAUSS (DUMMY)

This function generates e gauselan distribution of

unit standard deviation.

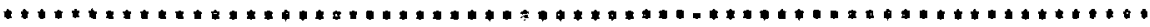

intęer seed

common seed

PARAMETER PI-3. 1415926

TEMP1-ŞRTT $(-2$. "ALOG (RANDOM $(.00001,1))$,

TEMPZ $=$ COS $(2$. PI* RANDOM $(0,1)$.

GAUSS-TFMPI- IEMPZ

RETIJRN

END 
Subroutine assign(m, 1ndex, overflow, $x \max , \operatorname{sm1n}, d x, x, n g e a)$

(2)

c

c

integer ngen(m)

Integer overflow

$\mathrm{c}$

$\mathrm{c}$

c

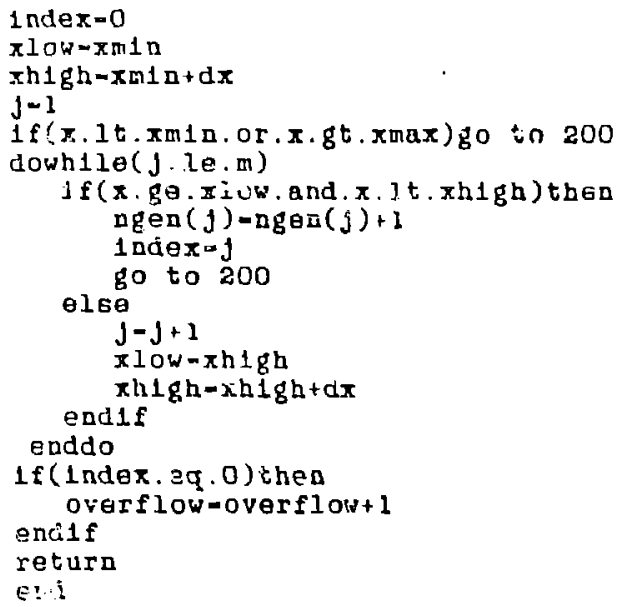


This report was prepared as an account of work sponsored by the Innited States Government. Neither the Unitad States nor the United States Departinent of Energy, nor any of their employees, makes any warranty, express or implied, or assumes any legal liability or responsibility for the accuracy, completeness, or usefulness of any information, apparatus, product, or process disclosed, or represents that its use would not infringe privately owned rights. Reference herein to any specific cominercial product, process, or service hy trade name, inark manufacturer, or otherwise, does not recessarily constitute or imply its endorsement, recommendation, or favoring by the United States Government or any agency thereof. The view and opinions of authors expressed herein do not necessarily state or reflect those of the United States Government or any agency thereof.

\section{PATENT STATUS}

This technical report is being transmitted in advance of D.0.E. patent clearance and no further dissemination or publication shall be made of the report without prior approval of the 0.0.E. Patent Counsel.

\section{TECHNICAL STATUS}

This technical report is being transmitted in advance of 0.0.E. review and no further dissemination or publication shall be made of the report without prior approval of the D.D.E. Project/Program Manager. 